
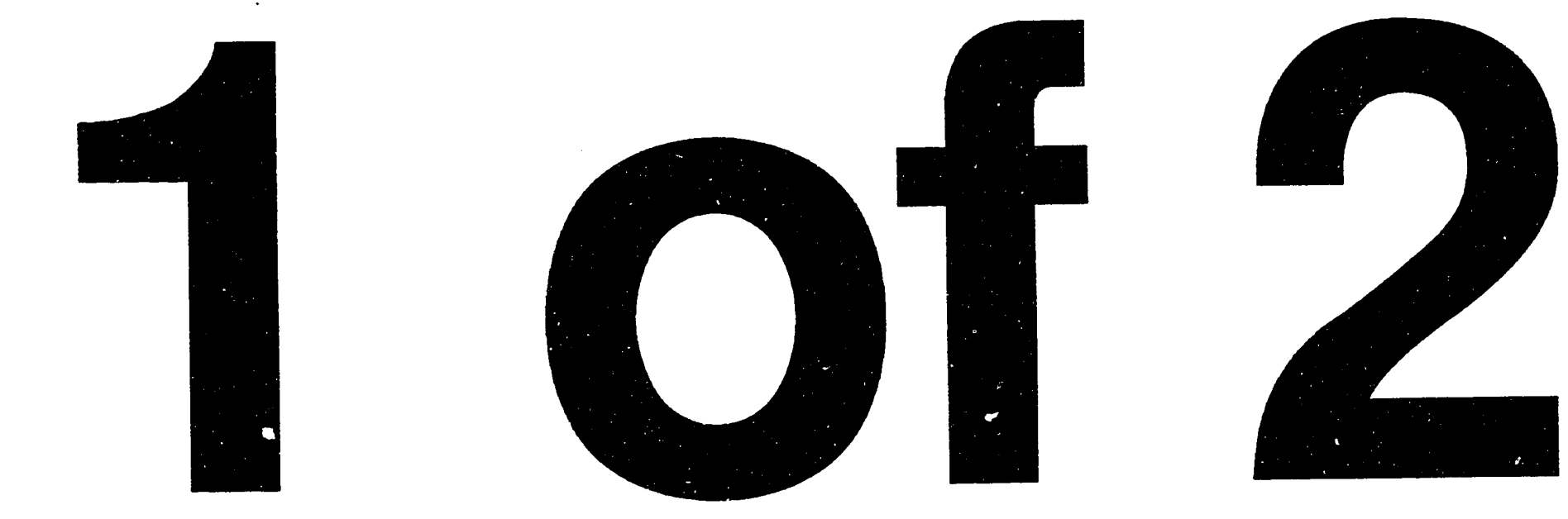
LBL-34738

\title{
Properties of W + Jet Events in Proton-Antiproton Collisions at $1.8 \mathrm{TeV}$
}

\author{
Robert Brian Drucker \\ Ph.D. Thesis \\ Department of Physics \\ University of California, Berkeley, CA 94720 \\ and \\ Lawrence Berkeley Laboratory \\ University of California, Berkeley, CA 94720
}

November 22, 1993

\begin{abstract}
W boson + QCD Jet events, produced in $1.8 \mathrm{TeV}$ proton-antiproton collisions and measured by the Collider Detector at Fermilab (CDF), were used to measure the center-of-mass production angle of the $W+$ jet system, and were also used. to place limits on the production of excited quark states. The center-of-mass production angular distribution agrees well with leading order and next-to-leading order QCD predictions. Excited quark states were searched for in the reaction $q+g \rightarrow q^{*} \rightarrow q+W$. Upper limits on the $q^{*}$ cross section, as a function of the $q^{*}$ mass, are shown. Comparison with a theoretical prediction for $q^{*}$ production excludes excited quark states with a mass in the range $150-530 \mathrm{GeV} / \mathrm{c}^{2}$, at $95 \%$ confidence.
\end{abstract}

This work was supported by the Director, Office of Energy Research, Office of High Energy and Nuclear Physics, Division of High Energy Physics, of the U.S.

Department of Energy under contract DE-AC03-76SF00098. 


\section{Properties of W + Jet Events in Proton-Antiproton Collisions at $1.8 \mathrm{TeV}$ \\ Copyright @ 1993 \\ Robert Brian Drucker}

The United States Department of Energy has the right to use this thesis for any purpose whatsoever including the right to reproduce all or any part thereof. 
This thesis is dedicated to my wife Sharon 


\section{Acknowledgements}

I would like to begin by thanking the CDF collaboration. I have found that there are many advantages to working with such a large group of physicists as is CDF. I was impressed by the broad spectrum of abilities represented, and the feeling of collaboration that was always present. This measurement would not have been possible without the incredible amount of work done by CDF to build the detector, record and understand the data. Special thanks to Les Nakae for his many useful comments on the first draft of part of this thesis, and for his friendship during the last few years. Robert Harris deserves many thanks. He was responsible for CDF's search for excited quarks in the photon channel. Our exchange of ideas and suggestions was very helpful to me. Special thanks to Brian Winer, who did much of the original work to define CDF's W boson sample, for answering my many questions when I was just starting to use this event sample.

I would also like to thank my local group here at Lawrence Berkeley Laboratory. They were always willing to hear the current state of my analysis and offered many helpful suggestions. Special thanks to Bob Ely, who supervised me during the first two years of my membership in CDF.

I am very grateful to my thesis advisor, Marjorie Shapiro. We spent many hours together discussing this analysis. I am very thankful for the concern that she has shown for me, and for all the graduate students around her. Marge has an inspiring intuition for physics, and I have learned inuch from her about what it means to be a careful scientist.

Most of all, I want to acknowledge the one person who has worked just as hard as I have in order to make this thesis a reality-my wife Sharon Drucker. During my career as a student, her love and support have always been there. She made our apartment a home, and our daughter could not have been blessed with a more wonderful mother. In many ways, this accomplishment is as much hers as it is mine.

Finally, I wish that I could thank both of my parents. For as long as I can remember I have wanted to be a scientist. Their love and encouragement must be largely responsible for my finally achieving this goal. I only hope, for my daughter's sake, that they're loving parenting has become a part of me. 


\section{The CDF Collaboration}

F. Abe ${ }^{(13)}$ M. Albrow, ${ }^{(7)}$ D. Amidei, ${ }^{(16)}$ C. Anway-Wiese, ${ }^{(4)}$ G. Apollinari, ${ }^{(26)}$ H. Areti, ${ }^{(7)}$ P. Auchincloss, ${ }^{(25)}$ F. Azfar, ${ }^{(21)}$ P. Azzi, ${ }^{(20)}$ N. Bacchetta, ${ }^{(18)}$ W. Badgett, ${ }^{(16)}$ M. W. Bailey, ${ }^{(24)}$ J. Bao, ${ }^{(33)}$ P. de Barbaro, ${ }^{(25)}$ A. BarbaroGaltieri, ${ }^{(14)}$ V. E. Barnes, ${ }^{(24)}$ B. A. Barnett, ${ }^{(12)}$ P. Bartalini, ${ }^{(23)}$ G. Bauer, ${ }^{(15)}$ T. Baumann, ${ }^{(9)}$ F. Bedeschi, ${ }^{(23)}$ S. Behrends, ${ }^{(2)}$ S. Belforte,${ }^{(23)}$ G. Bellettini, ${ }^{(23)}$ J. Bellinger, ${ }^{(32)}$ D. Benjamin, ${ }^{(31)}$ J. Benlloch, ${ }^{(15)}$ D. Benton, ${ }^{(21)}$ A. Beretvas, ${ }^{(7)}$ J. P. Berge, ${ }^{(7)}$ A. Bhatti, ${ }^{(26)}$ K. Biery, ${ }^{(11)}$ M. Binkley, ${ }^{(7)}$ F. Bird, ${ }^{(28)}$ D. Bisello, ${ }^{(20)}$ R. E. Blair, ${ }^{(1)}$ C. Blocker, ${ }^{(28)}$ A. Bodek, ${ }^{(25)}$ V. Bolognesi, ${ }^{(23)}$ D. Bortoletto, ${ }^{(24)}$ C. Boswell, ${ }^{(12)}$ T. Boulos, ${ }^{(14)}$ G. Brandenburg, ${ }^{(9)}$ E. BuckleyGeer, ${ }^{(7)}$ H. S. Budd, ${ }^{(25)}$ K. Burkett, ${ }^{(16)}$ G. Busetto, ${ }^{(20)}$ A. Byon-Wagner, ${ }^{(7)}$ K. L. Byrum, ${ }^{(1)}$ C. Campagnari, ${ }^{(7)}$ M. Campbell, ${ }^{(16)}$ A. Caner, ${ }^{(7)}$ W. Carithers, ${ }^{(14)}$ D. Carlsmith, ${ }^{(32)}$ A. Castro, ${ }^{(20)}$ Y. Cen, ${ }^{(21)}$ F. Cervelli, (23) J. Chapman, ${ }^{(16)}$ G. Chiarelli, ${ }^{(8)}$ T. Chikamatsu, ${ }^{(30)}$ S. Cihangir, ${ }^{(7)}$ A. G. Clark, ${ }^{(23)}$ M. Cobal, ${ }^{(23)}$ M. Contreras, ${ }^{(5)}$ J. Cooper, ${ }^{(7)}$ M. Cordelli, ${ }^{(8)}$ D. P. Coupal, ${ }^{(28)}$ D. Crane, ${ }^{(7)}$ J. D. Cunningham, ${ }^{(2)}$ T. Dâniels, ${ }^{(15)}$ F. DeJongh, ${ }^{(7)}$ S. Dell'Agnello, ${ }^{(23)}$ M. Dell'Orso, ${ }^{(23)}$ L. Demortier, ${ }^{(26)}$ B. Denby, ${ }^{(7)}$ M. Deninno, ${ }^{(3)}$ P. F. Derwent, ${ }^{(16)}$ T. Devlin, ${ }^{(27)}$ M. Dickson, ${ }^{(25)}$ S. Donati, ${ }^{(23)}$ J. P. Done, ${ }^{(29)}$ R. B. Drucker, ${ }^{(14)}$ A. Dunn, ${ }^{(16)}$ K. Einsweiler, ${ }^{(14)}$ J. E. Elias, ${ }^{(7)}$ R. Ely, ${ }^{(14)}$ E. Engels, Jr., ${ }^{(22)}$ S. Eno, ${ }^{(5)}$ D. Errede, ${ }^{(10)}$ S. Errede, ${ }^{(10)}$ A. Etchegoyen, ${ }^{(7 a)}$ Q. Fan, ${ }^{(25)}$ B. Farhat,${ }^{(15)}$ I. Fiori, ${ }^{(3)}$ B. Flaugher, ${ }^{(7)}$ G. W. Foster, ${ }^{(7)}$ M. Franklin, ${ }^{(9)}$ M. Frautschi, ${ }^{(18)}$ J. Freeman, ${ }^{(7)}$ J. Friedman, ${ }^{(15)}$ H. Frisch, ${ }^{(5)}$ A. Fry, ${ }^{(28)}$ T. A. Fuess, ${ }^{(28)}$ Y. Fukui, ${ }^{(13)}$ S. Funaki, ${ }^{(30)}$ A. F. Garfinkel, ${ }^{(24)}$ S. Geer, ${ }^{(7)}$ D. W. Gerdes, ${ }^{(16)}$ P. Giannetti, ${ }^{(23)}$ N. Giokaris, ${ }^{(26)}$ P. Giromini, ${ }^{(8)}$ L. Gladney, ${ }^{(21)}$ D. Glenzinski, ${ }^{(12)}$ M. Gold, ${ }^{(18)}$ J. Gonzalez, ${ }^{(21)}$ A. Gordon, ${ }^{(9)}$ A. T. Goshaw, ${ }^{(6)}$ K. Goulianos, ${ }^{(26)}$ H. Grassmann, ${ }^{(28)}$ A. Grewal, ${ }^{(21)}$ G. Grieco, ${ }^{(23)}$ L. Groer, ${ }^{(27)}$ C. Grosso-Pilcher, ${ }^{(5)}$ C. Haber ${ }^{(14)}$ S. R. Hahn, ${ }^{(7)}$ R. Hamilton, ${ }^{(9)}$ R. Handler, ${ }^{(32)}$ R. M. Hans, ${ }^{(33)}$ K. Hara, ${ }^{(30)}$ B. Harral, ${ }^{(21)}$ R. M. Harris, ${ }^{(7)}$ S. A. Hauger, ${ }^{(6)}$ J. Hauser, ${ }^{(4)}$ C. Hawk, ${ }^{(27)}$ J. Heinrich, ${ }^{(21)}$ D. Hennessy, ${ }^{(6)}$ R. Hollebeek, ${ }^{(21)}$ L. Holloway, ${ }^{(10)}$ A. Hölscher, ${ }^{(11)}$ S. Hong, ${ }^{(16)}$ G. Houk, ${ }^{(21)}$ P. Hu, (22) B. T. Huffman, ${ }^{(22)}$ R. Hughes, ${ }^{(25)}$ P. Hurst, ${ }^{(9)}$ J. Huston, ${ }^{(17)} \mathrm{J}$. Huth, ${ }^{(7)} \mathrm{J}$. Hylen, ${ }^{(7)} \mathrm{M}$. Incagli, ${ }^{(23)}$ J. Incandela, ${ }^{(7)} \mathrm{H}$. Iso, ${ }^{(30)} \mathrm{H}$. Jensen, ${ }^{(7)}$ C. P. Jessop, ${ }^{(9)}$ U. Joshi, ${ }^{(7)}$ R. W. Kadel, ${ }^{(14)}$ E. Kajfasz, ${ }^{(7)}$ T. Kamon, ${ }^{(29)}$ T. Kaneko, ${ }^{(30)}$ D. A. Kardelis, ${ }^{(10)}$ H. Kasha, ${ }^{(33)}$ Y. Kato, ${ }^{(19)}$ L. Keeble, ${ }^{(29)}$ R. D. Kennedy, ${ }^{(27)}$ R. Kephart, ${ }^{(7)}$ P. Kesten, ${ }^{(14)}$ D. Kestenbaum, ${ }^{(9)}$ R. M. Keup, ${ }^{(10)}$ H. Keutelian, ${ }^{(7)}$ F. Keyvan, ${ }^{(4)}$ D. H. Kim, ${ }^{(7)}$ S. B. Kim, ${ }^{(16)}$ S. H. Kim, ${ }^{(30)}$ Y. K. Kim, ${ }^{(14)}$ L. Kirsch, ${ }^{(2)}$ P. Koehn, ${ }^{(25)}$ K. Kondo, ${ }^{(30)}$ J. Konigsberg, ${ }^{(9)}$ S. Kopp, ${ }^{(5)}$ K. Kordas, ${ }^{(11)}$ W. Koska, ${ }^{(7)}$ E. Kovacs, ${ }^{(7 a)}$ M. Krasberg, ${ }^{(16)}$ S. E. Kuhlmann, ${ }^{(1)}$ E. Kuns, ${ }^{(27)}$ A. T. Laasanen, ${ }^{(24)}$ S. Lammel, ${ }^{(4)}$ J. I. Lamoureux, ${ }^{(32)}$ T. LeCompte, ${ }^{(10)}$ S. Leone, ${ }^{(23)} \mathrm{J}$. D. Lewis, ${ }^{(7)}$ P. Limon, ${ }^{(7)}$ M. Lindgren, ${ }^{(4)}$ T. M. Liss, ${ }^{(10)}$ N. Lockyer, ${ }^{(21)}$ O. Long, ${ }^{(21)}$ 
M. Loreti, ${ }^{(20)}$ E. H. Low, ${ }^{(21)}$ D. Lucchesi, ${ }^{(23)}$ C. B. Luchini, ${ }^{(10)}$ P. Lukens, ${ }^{(7)}$ P. Maas, ${ }^{(32)}$ K. Maeshima, ${ }^{(7)}$ A. Maghakian, ${ }^{(26)}$ M. Mangano, ${ }^{(23)}$ J. Mansour, ${ }^{(17)}$ M. Mariotti, ${ }^{(23)}$ J. P. Marriner, ${ }^{(7)}$ A. Martin ${ }^{(10)}$ J. A. J. Matthews, ${ }^{(18)}$ R. Mattingly, ${ }^{(2)}$ P. McIntyre, ${ }^{(29)}$ P. Melese, ${ }^{(26)}$ A. Menzione, ${ }^{(23)}$ E. Meschi, ${ }^{(23)}$ S. Mikamo, ${ }^{(13)}$ M. Miller, ${ }^{(5)}$ T. Mimashi, ${ }^{(30)}$ S. Miscetti, ${ }^{(8)}$ M. Mishina, ${ }^{(13)}$ H. Mitsushio, ${ }^{(30)}$ S. Miyashita, ${ }^{(30)}$ Y. Morita, ${ }^{(13)}$ S. Moulding, ${ }^{(26)}$ J. Mueller, ${ }^{(27)}$ A. Mukherjee, ${ }^{(7)}$ T. Muller, ${ }^{(4)}$ L. F. Nakae, ${ }^{(28)}$ I. Nakano, ${ }^{(30)}$ C. Nelson, ${ }^{(7)}$ D. Neuberger, ${ }^{(4)}$ C. Newman-Holmes, ${ }^{(7)}$ L. Nodulman, ${ }^{(1)}$ S. Ogawa, ${ }^{(30)}$ K. E. Ohl, ${ }^{(3)}$ R. Oishi, ${ }^{(30)}$ T. Okusawa, ${ }^{(19)}$ C. Pagliarone, ${ }^{(23)}$ R. Paoletti, ${ }^{(23)}$ V. Papadimitriou, ${ }^{(7)}$ S. Park, ${ }^{(7)}$ J. Patrick, ${ }^{(7)}$ G. Pauletta, ${ }^{(23)}$ L. Pescara, ${ }^{(20)}$ M. D. Peters ${ }^{(14)}$ T. J. Phillips, ${ }^{(6)}$ G. Piacentino, ${ }^{(3)}$ M. Pillai, ${ }^{(25)}$ R. Plunkett, ${ }^{(7)}$ L. Pondrom, ${ }^{(32)}$ N. Produit, ${ }^{(14)}$ J. Proudfoot, ${ }^{(1)}$ F. Ptohos, ${ }^{(9)}$ G. Punzi, ${ }^{(23)}$

K. Ragan, ${ }^{(11)}$

F. Rimondi, ${ }^{(3)}$ L. Ristori, ${ }^{(23)}$ M. Roach-Bellino, ${ }^{(31)}$ W. J. Robertson, ${ }^{(6)}$ T. Rodrigo, ${ }^{(7)}$ J. Romano, ${ }^{(5)}$ L. Rosenson, ${ }^{(15)}$ W. K. Sakumoto, ${ }^{(25)}$ D. Saltzberg, ${ }^{(5)}$ A. Sansoni, ${ }^{(8)}$ V. Scarpine, ${ }^{(29)}$ A. Schindler, ${ }^{(14)}$ P. Schlabach, ${ }^{(9)}$ E. E. Schmidt, ${ }^{(7)}$ M. P. Schmidt, ${ }^{(33)}$ O. Schneider, ${ }^{(14)}$ G. F. Sciacca, ${ }^{(23)}$ A. Scribano, ${ }^{(23)}$ S. Segler ${ }^{(7)}$ S. Seidel ${ }^{(18)}$ Y. Seiya, ${ }^{(30)}$ G. Sganos, ${ }^{(11)}$ M. Shapiro, ${ }^{(14)}$ N. M. Shaw,${ }^{(24)}$ Q. Shen, ${ }^{(24)}$ P. F. Shepard, ${ }^{(22)}$ M. Shimojima, ${ }^{(30)}$ M. Shochet, ${ }^{(5)}$ J. Siegrist, ${ }^{(28)}$ A. Sill, ${ }^{(72)}$ P. Sinervo, ${ }^{(11)}$ P. Singh, ${ }^{(22)}$ J. Skarha, ${ }^{(12)}$ K. Sliwa, ${ }^{(31)}$ D. A. Smith, ${ }^{(23)}$ F. D. Snider, ${ }^{(12)}$ L. Song, ${ }^{(7)}$ T. Song, ${ }^{(16)}$ J. Spalding, ${ }^{(7)}$ P. Sphicas, ${ }^{(15)}$ A. Spies, ${ }^{(12)}$ L. Stanco, ${ }^{(20)}$ J. Steele,${ }^{(32)}$ A. Stefanini, ${ }^{(23)}$ J. Strait,${ }^{(7)}$ G. Sullivan, ${ }^{(5)}$ K. Sumorok, ${ }^{(15)}$ R. L. Swartz, Jr., ${ }^{(10)}$ T. Takahashi, ${ }^{(19)}$ K. Takikawa, ${ }^{(30)}$ F. Tartarelli, ${ }^{(23)}$ Y. Teramoto, ${ }^{(19)}$ S. Tether, ${ }^{(15)}$ D. Theriot, ${ }^{(7)}$ J. Thomas, ${ }^{(28)}$ R. Thun, ${ }^{(16)}$ M. Timko, ${ }^{(31)}$ P. Tipton, ${ }^{(25)}$ A. Titov, ${ }^{(26)}$ S. Tkaczyk, ${ }^{(7)}$ A. Tollestrup, ${ }^{(7)}$ J. Tonnison, ${ }^{(24)}$ J. Tseng, ${ }^{(12)}$ M. Turcotte, ${ }^{(28)}$ N. Turini, ${ }^{(3)}$ N. Uemura, ${ }^{(30)}$ F. Ukegawa, ${ }^{(21)}$ G. Unal, ${ }^{(21)}$ S. Vejcik, III, ${ }^{(16)}$ R. Vidal, ${ }^{(7)}$ M. Vondracek, ${ }^{(10)}$ R. G. Wagner, ${ }^{(1)}$ R. L. Wagner, ${ }^{(7)}$ N. Wainer,${ }^{(7)}$ R. C. Walker, ${ }^{(25,}$ J. Wang, ${ }^{(5)}$ Q. F. Wang, ${ }^{(26)}$ A. Warburton, ${ }^{(11)}$ G. Watts ${ }^{(25)}$ T. Watts, ${ }^{(27)}$ R. Webb, ${ }^{(29)}$ C. Wendt, ${ }^{(32)} \mathrm{H}$. Wenzel, ${ }^{(7 a)}$ W. C. Wester, III, ${ }^{(14)}$ T. Westhusing, ${ }^{(10)}$ A. B. Wicklund, ${ }^{(1)}$ E. Wicklund, ${ }^{(7)}$ R. Wilkinson, ${ }^{(21)}$ H. H. Williams, ${ }^{(21)}$ B. L. Winer, ${ }^{(25)} \mathrm{J}$. Wolinski, ${ }^{(29)} \mathrm{D}$. Y. Wu, ${ }^{(16)} \mathrm{X}$. Wu ${ }^{(23)} \mathrm{J}$. Wyss, ${ }^{(20)} \mathrm{A}$. Yagil, ${ }^{(7)}$ W. Yao, ${ }^{(14)}$ K. Yasuoka, ${ }^{(30)}$ Y. Ye, ${ }^{(11)}$ G. P. Yeh, ${ }^{(7)}$ M. Yin, ${ }^{(6)}$ J. Yoh, ${ }^{(7)}$ T. Yoshida, ${ }^{(19)}$ D. Yovanovitch, ${ }^{(7)}$ I. Yu, ${ }^{\left({ }^{(3)}\right)}$ J. C. Yun, ${ }^{(7)}$ A. Zanetti, ${ }^{(23)}$ F. Zetti, ${ }^{(23)}$ S. Zhang, ${ }^{(15)}$ W. Zhang, ${ }^{(21)}$ G. C. Zucchelli, ${ }^{(23)}$ and S. Zucchelli ${ }^{(3)}$

(1) Argonne National Laboratory, Argonne, Illinois 60439

(2) Brandeis University, Waltham, Massachusetts 02254

(3) Istituto Nazionale di Fisica Nucleare, University of Bologna, I-40126 Bologna, Italy

(4) University of California at Los Angeles, Los Angeles, California 90024

(5) University of Chicago, Chicago, Illinois 60637

(6) Duke University, Durham, North Carolina 27708 
(7) Fermi National Accelerator Laboratory, Batavia, Illinois 60510

(8) Laboratori Nazionali di Frascati, Istituto Nazionale di Fisica Nucleare, I-00044 Frascati, Italy

(9) Harvard University, Cambridge, Massachusetts 02138

(10) University of Illinois, Urbana, Illinois 61801

(11) Institute of Particle Physics, McGill University, Montreal H3A 2T8, and U'niversity of Toronto, Toronto M5S 1A7, Canada

(12) The Johns Hopkins University, Baltimore, Maryland 21218

(13) National Laboratory for High Energy Physics (KEK), Tsukuba, Ibaraki 305, Japan

(14) Lawrence Berkeley Laboratory, Berkeley, California 94720

(15) Massachusetts Institute of Technology, Cambridge, Massachusetts 02139

(16) University of Michigan, Ann Arbor, Michigan 48109

(17) Michigan State University, East Lansing, Michigan 48824

(18) University of New Mexico, Albuquerque, New Mexico 87131

(19) Osaka City University, Osaka 588, Japan

(20) Uni versita di Padova, Instituto Nazionale di Fisica Nucleare, Sezione di Padova, I-35131 Padova, Italy

(21) University of Pennsylvania, Philadelphia, Pennsylvania 19104

(22) University of Pittsburgh, Pittsburgh, Pennsylvania 15260

(23) Istituto Nazionale di Fisica Nucleare, University and Scuola Normale

Superiore of Pisa, I-56100 Pisa, Italy

(24) Purdue University, West I afayette, Indiana 47907

(25) University of Rochester, Rochester, New York 14627

(26) Rockefeller University, New York, New York 10021

(27) Rutgers University, Piscataway, New Jersey 08854

(28) Supercorducting Super Collider Laboratory, Dallas, Texas 75237

(29) Texas A\&M University, College Station, Texas 77843

(30) University of Tsukuba, Tsukuba, Ibaraki 305, Japan

(31) Tufts University, Medford, Massachusetts 02155

(32) University of Wisconsin, Madison, Wisconsin 53706

(33) Yale University, New Haven, Connecticut 065il

This work was supported by the Director, Office of Energy Research, Office of High Energy and Nuclear Physics, Division of High Energy Physics, of the U.S. Department of Energy under contract DE-AC03-76SF00098. 


\section{Contents}

Acknowledgements

1 Introduction 1

1.1 The Standard Model ... . . . . . . . . . . . . . . 1

1.1.1 The Known Forces ................ 1

1.1.2 The Known Particles ............... 3

1.2 This Thesis ..................... 4

2 Theory 6

2.1 Proton-Antiproton Collisions .............. 6

2.2 Coordinate System and Units .............. 8

2.3 QCD Perturbation Theory ............... 10

2.3.1 Momentum Scale .............. 10

2.3 .2 Singularities ..................... 11

2.3.3 Parton Fragmentation .............. 12

2.3 .4 Intrinsic $\mathrm{K}_{t} \ldots \ldots \ldots \ldots 12 \ldots \ldots \ldots$

2.4 W Production at Hadron Colliders .............. 12

3 Analysis Overview 15

3.1 Properties of $\mathrm{W}+$ Jet Events . . . . . . . . . . . . 15

3.1.1 Collins-Soper Reference Frame . . . . . . . . . . 15

3.1 .2 Center-of-mass Variables . . . . . . . . . . . 16

3.1.3 W Rest Frame. . . . . . . . . . . . . . 18

3.2 The Undetected Neutrino . . . . . . . . . . . . 22

3.2.1 Determination of the Neutrino Momentum . . . . . . . . 22

3.2.2 Selection of One Neutrino Solution . . . . . . . . 25 
viii

3.3 Acceptance Issues . . . . . . . . . . . . . . . . 26

3.4 Transformation to True $\operatorname{Cos} \theta^{*} \ldots \ldots \ldots$

3.5 Normalization of the $\operatorname{Cos} \theta^{*}$ Distribution . . . . . . . . . 32

3.6 Summary .......................... 32

4 CDF Detector 34

4.1 Fermilab Collider . . . . . . . . . . . . . . . 36

4.2 Vertex Time Projection Chamber . . . . . . . . . . . . 36

4.3 Central Tracking Chamber . . . . . . . . . . . . 38

4.4 Magnet ......................... 42

4.5 Calorimetry ....................... 42

4.5.1 Central Calorimeters . . . . . . . . . . . . 42

4.5 .2 Plug Calorimeters . . . . . . . . . . . . . 48

4.5.3 Forward Calorimeters . . . . . . . . . . . . . . 50

4.6 Central Muor Chambers . . . . . . . . . . . . . 54

4.7 Data Acquisition System . . . . . . . . . . . . . 58

5 Event Reconstruction and Selection $\quad \mathbf{6 0}$

5.1 Electrons. . . . . . . . . . . . . . . . . . . 60

5.1 .1 Electron Clustering . . . . . . . . . . . . 60

5.1 .2 Electron Identification Cuts ............ 61

5.1.3 Electron Corrections ............... 65

5.1.4 Electron Eta Acceptance Correction . . . . . . . . . . 67

5.2 Muons ......................... 67

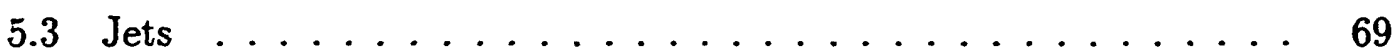

5.3 .1 Jet Corrections ................. 70

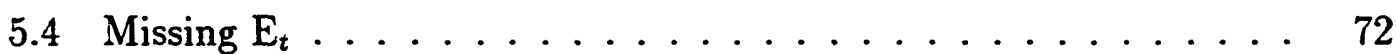

5.4.1 Missing $\mathrm{E}_{t}$ Corrections . . . . . . . . . . . 74

5.4.1.1 Electron energy corrections to $\overrightarrow{\mathbb{E}}_{t} \ldots \ldots . \ldots 74$

5.4.1.2 Jet energy corrections to $Z_{t} \ldots \ldots \ldots 74$

5.4.1.3 Muon corrections to $\mathbb{Z}_{t} \ldots \ldots \ldots 75$

5.4.1.4 Unclustered-energy corrections to $Z_{t} \ldots \ldots 75$

$5.5 \mathrm{~W}+$ Jet $\rightarrow \mathrm{e}+\nu+$ Jet Event Selection . . . . . . . . 75

$5.6 \mathrm{~W}+$ Jet $\rightarrow \mu+\nu+$ Jet Event Selection . . . . . . . . . 78 
6 Simulation Tools and Comparison with Data 81

6.1 Parton Level Simulation . . . . . . . . . . . . . . . . 81

6.1.1 vecBos Event Generator . . . . . . . . . . . . . 82

6.1 .2 QDJTMC Detector Simulation ........... 82

6.2 Hadron Level Simulation . . . . . . . . . . . . . 85

6.2.1 Pythia Event Generator .............. 85

6.2.2 QFL Detector Simulation . . . . . . . . . . . 85

6.3 General Comparison with CDF Data . . . . . . . . . 86

7 Analysis $\quad 94$

7.1 W Mass Constraint . . . . . . . . . . . . . . . . . . 94

7.1.1 Events Failing the W Mass Constraint . . . . . . . . . 94

$7.2 \mathrm{~W}^{+}$Event Inversion . . . . . . . . . . . . . 96

7.3 Solution Selection . . . . . . . . . . . . . 96

7.4 Center-of-mass Variables . . . . . . . . . . . . . . . 98

$7.5 \operatorname{Cos} \theta_{\mathrm{s}}^{*}$ Region Measured ............... 98

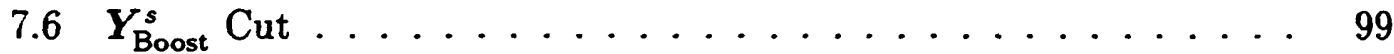

$7.7 \mathrm{M}_{\mathrm{s}}^{*} \mathrm{Cut} \ldots \ldots \ldots \ldots \ldots \ldots \ldots \ldots$

7.8 Residual Detector Effects and $\operatorname{Cos} \theta_{\mathrm{s}}^{*}$ Resolution . . . . . . . 101

$\begin{array}{llr}8 & \text { Backgrounds } & \mathbf{1 1 0}\end{array}$

8.1 Electroweak Backgrounds . . . . . . . . . . . . . 110

8.1.1 $W \rightarrow \tau \nu \rightarrow e \nu \nu \nu \ldots \ldots \ldots \ldots \ldots \ldots \ldots \ldots$

$8.1 .2 Z \rightarrow \mathrm{e}^{+} \mathrm{e}^{-} \ldots \ldots \ldots \ldots \ldots \ldots \ldots \ldots \ldots$

$8.1 .3 Z \rightarrow \tau^{+} \tau^{-} \ldots \ldots \ldots \ldots \ldots \ldots$

8.2 QCD Background . . . . . . . . . . . . . . 114

8.3 Top Quark Background . . . . . . . . . . . . . . . . . 119

8.4 Summary of Backgrounds . . . . . . . . . . . . 119

9 Systematic Uncertainties $\quad 121$

9.1 Jet Energy Scales . . . . . . . . . . . . . . . . . . . 121

9.1.1 Absolute Jet Energy Scale . . . . . . . . . . . . . 121

9.1.2 Relative Jet Energy Scale . . . . . . . . . . . . 122

9.2 Backgrounds . . . . . . . . . . . . . . . . 122

9.3 Parton Distribution Functions . . . . . . . . . . . . 125 
$9.4 Q^{2}$ Scale . . . . . . . . . . . . . . . . 128

9.5 W Charge . . . . . . . . . . . . . . . . 128

9.6 Intrinsic $\mathrm{K}_{t} \ldots \ldots \ldots \ldots \ldots \ldots \ldots \ldots$

9.7 Summary of Systematics . . . . . . . . . . . . . 131

10 Results and Comparison with Theory 133

10.1 Method for Cornparing with Theory . . . . . . . . . . 133

10.2 Normalization of $\operatorname{Cos} \theta_{\mathrm{s}}^{*} \ldots \ldots \ldots . . \ldots 135$

10.3 Measured $\operatorname{Cos} \theta_{\mathrm{s}}^{*}$ Distribution .............. 135

11 Search for Excited Quark States Decaying into W + Jet 140

11.1 Theory . . . . . . . . . . . . . . . . . 140

11.2 Previous Searches . . . . . . . . . . . . . 141

11.3 Data Samples . . . . . . . . . . . . . . . . 142

11.4 QSTAR Monte Carlo . . . . . . . . . . . . . . . 143

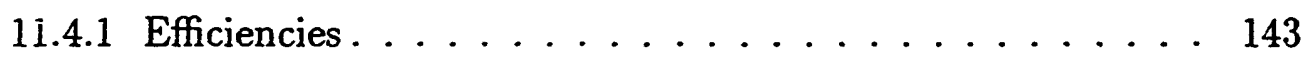

11.4.1.1 Electron Efficiencies ........... 143

11.4.1.2 Muon Efficiencies . . . . . . . . . . 145

11.4.1.3 Mass-dependent Efficiencies ... . . . . . 145

11.5 Analysis . . . . . . . . . . . . . . . . 146

11.5.1 Differences from the $\operatorname{Cos} \theta_{\mathrm{s}}^{*}$ Analysis . . . . . . . 146

11.5.1.1 Definition of "Selected" Solution for the $\boldsymbol{q}^{*}$ Search 148

11.5.2 Distributions of Selected $\mathrm{M}^{*} \ldots \ldots . . . . . .150$

11.6 Determination of the Excited Quark Limits . . . . . . . . . . 150

11.7 Systematics . . . . . . . . . . . . . . . . 157

11.7.1 Method for Folding Systematics into the Limits . . . . . 157

11.7.2 Monte Carlo Limit Predictions . . . . . . . . . . . . 158

11.7.3 Discussion of Each Systematic Uncertainty . . . . . . . 158

11.7.4 Summary of Systematic Uncertainties . . . . . . . . . 165

11.8 Excited Quark Limits . . . . . . . . . . . . . . . . 165

11.8.1 Mass Limit Studies . . . . . . . . . . . . . 167

11.9 Conclusions . . . . . . . . . . . . . . 169

$\begin{array}{lr}12 \text { Conclusions } & \mathbf{1 7 0}\end{array}$

$\begin{array}{ll}\text { Bibliography } & \mathbf{1 7 1}\end{array}$ 


\section{List of Tables}

1.1 The Standard Model Forces . . . . . . . . . . . . . . . . 2

1.2 The Standard Model Particles . . . . . . . . . . . . . 2

4.1 vTPC Specifications . . . . . . . . . . . . . . . 41

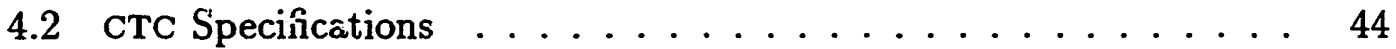

4.3 Central Calorimeter Specifications . . . . . . . . . . . . . 49

4.4 Plug Calorimeter Specifications _. . . . . . . . . . . 51

4.5 Forward Calorimeter Specifications . . . . . . . . . 55

4.6 CMU Specifications $\ldots \ldots \ldots \ldots \ldots \ldots \ldots \ldots$

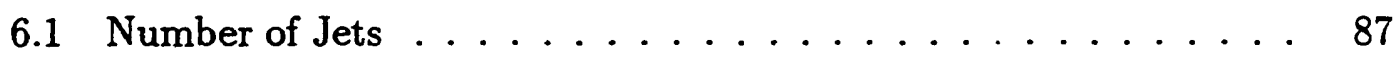

8.1 Summary of Backgrounds $\ldots \ldots \ldots \ldots \ldots$

9.1 Summary of $\cos \theta_{s}^{*}$ Uncertainties . . . . . . . . . 131

11.1 Branching Ratios for Excited Quarks . . . . . . . . . . 141

11.2 Electron Efficiencies. . . . . . . . . . . . . . . . . . 144

11.3 Muon Efficiencies . . . . . . . . . . . . . . . 145

11.4 Summary of Systematic Uncertainties . . . . . . . . . . . 165 
xii

\section{List of Figures}

2.1 Generic Hadron Collision . . . . . . . . . . . . 7

2.2 Parton Distribution Functions . . . . . . . . . . . . 9

2.3 Leading Order Feynman Diagrams . . . . . . . . . . 13

2.4 Typical W + Jet Event . . . . . . . . . . . . . . 14

3.1 Collins-Soper Reference Frame . . . . . . . . . . . . 17

$3.2 \mathrm{~W}+$ Jet Center-of-mass Frame . . . . . . . . . . . . 17

3.3 Parton Model $\cos \theta^{*} \ldots \ldots \ldots$. . . . . . . . . 19

3.4 Parton Model $\mathrm{M}^{*} \ldots \ldots \ldots 20$

3.5 Parton Model $Y_{\text {Boost }} \ldots \ldots \ldots 21$

3.6 W Rest Frame . . . . . . . . . . . . . . . . . 23

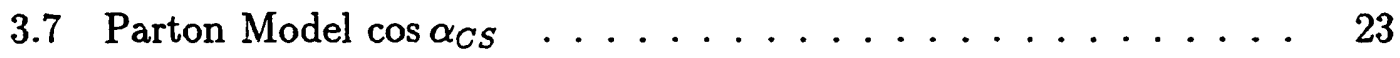

3.8 Parton Model $\phi_{C S} \ldots \ldots \ldots 24$

3.9 Parton Model $\cos \theta^{*}$ Solutions . . . . . . . . . . . . 27

3.10 Parton Model $\cos \theta_{s}^{*}$ Dependance on $\cos \theta^{*} \ldots \ldots 28$

3.11 Parton Model $\left|\cos \theta_{s}^{*}\right|$ Dependance on $\left|\cos \theta^{*}\right| \ldots \ldots 29$

3.12 Parton Model Acceptance in $\cos \alpha_{C S}-Y_{\text {Boost }}$ Plane . . . . . . . . 31

4.1 CDF Detector . . . . . . . . . . . . . . 35

4.2 Fermilab Accelerator Complex . . . . . . . . . . . 37

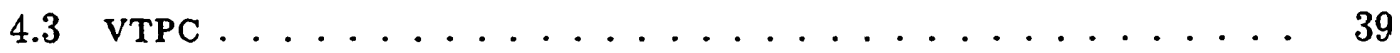

$4.4 \quad$ vTPC Event Display . . . . . . . . . . . . . . . 40

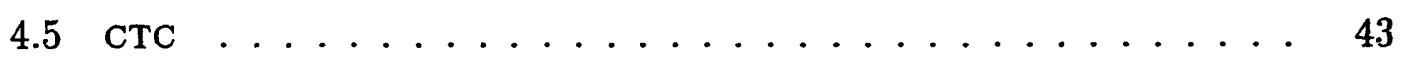

4.6 CTC Event Display . . . . . . . . . . . . . . 43

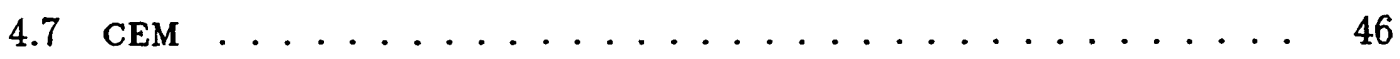

4.8 CEs .................................. 46

4.9 CHA Layer . . . . . . . . . . . . . . . . . 47 
4.10 CHA Module . . . . . . . . . . . . . 47

4.11 Plug Calorimeter Construction . . . . . . . . . . . 49

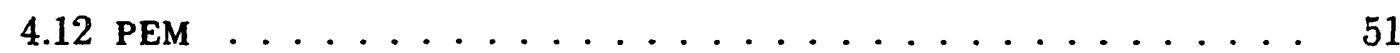

4.13 Forward Calorimeter Construction . . . . . . . . . . . 52

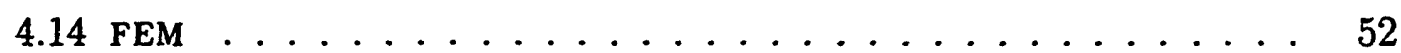

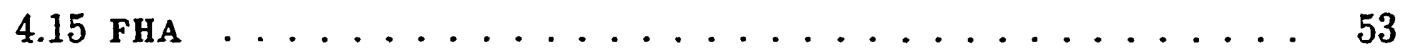

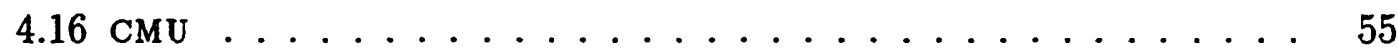

4.17 CMU Drift Cell . . . . . . . . . . . . . . 56

4.18 CMU Tower $\ldots \ldots \ldots \ldots \ldots \ldots \ldots \ldots \ldots \ldots$

5.1 Electron Identification Cuts . . . . . . . . . . . . . 62

5.2 Electron Identification Cuts continued $\ldots \ldots \ldots \ldots \ldots$

5.3 CEM face response map . . . . . . . . . . . . 66

5.4 Electron $\eta$ Acceptance . . . . . . . . . . . . . . . 68

5.5 Central Calorimeter Response to Low Energy Hadrons . . . . . 71

5.6 Jet Correction Function $\ldots \ldots \ldots \ldots \ldots \ldots$

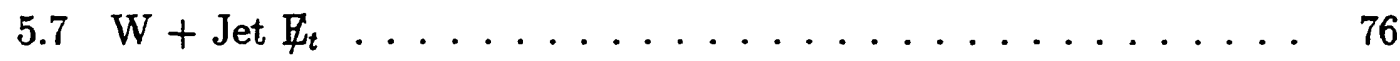

$5.8 \mathrm{~W}+$ Jet Transverse Mass $\ldots \ldots \ldots \ldots \ldots \ldots \ldots$

6.1 Underlying Event $\sum \mathrm{E}_{t} \ldots \ldots \ldots \ldots \ldots \ldots \ldots$

6.2 Electron $\mathrm{P}_{t} \ldots \ldots \ldots \ldots \ldots \ldots \ldots \ldots \ldots \ldots$

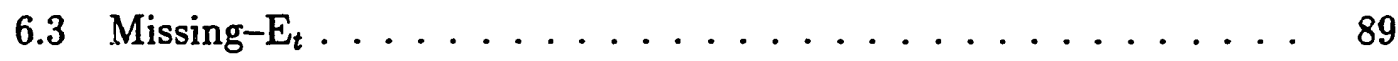

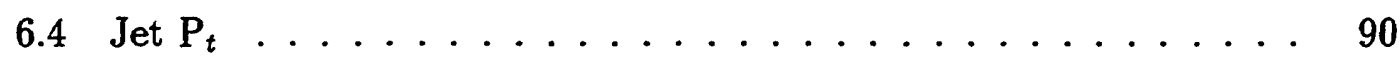

$6.5 W$ Boson $\mathrm{P}_{t} \ldots \ldots \ldots \ldots \ldots \ldots \ldots \ldots \ldots \ldots$

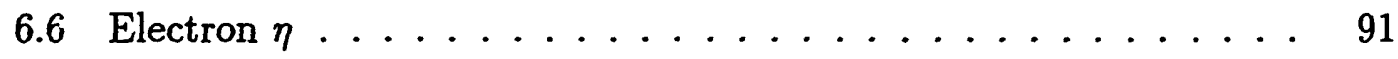

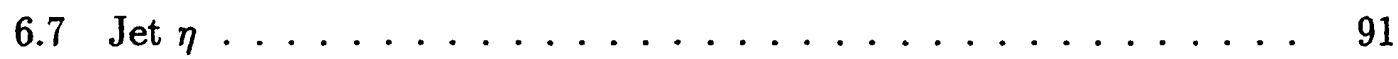

6.8 Underlying Event $P_{t} \ldots \ldots \ldots \ldots \ldots \ldots \ldots \ldots \ldots$

$6.9 \mathrm{~W}+$ Jet Transverse Momentum . . . . . . . . . . . . 92

$6.10 \mathrm{~W}$ Transverse Mass . . . . . . . . . . . . . . . . . 93

6.11 Neutrino $\mathrm{P}_{z} \ldots \ldots \ldots \ldots \ldots \ldots \ldots \ldots \ldots$

7.1 True Minus False $\mathrm{P}_{z}^{\nu}$ (Constraint Failures) $\ldots \ldots \ldots \ldots$

7.2 Maximum $\cos \theta_{s}^{*}$ Cut . . . . . . . . . . 100

7.3 Minimum Jet $P_{t}$ vs. $\cos \theta_{s}^{*} \ldots \ldots \ldots \ldots \ldots \ldots 102$

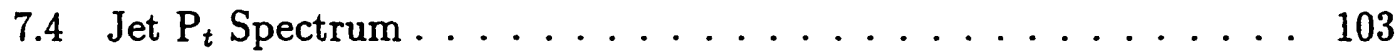


xiv

$7.5 \cos \theta_{s}^{*}$ Acceptance . . . . . . . . . . . . . 105

$7.6 \cos \theta_{s}^{*}$ Acceptance without Constraint Failures . . . . . . . 106

7.7 Simulated versus Generated $\cos \theta_{s}^{*} \ldots \ldots \ldots 10 . . \ldots 1$

$7.8 \cos \theta_{s}^{*}$ Resolution . . . . . . . . . . . . . 108

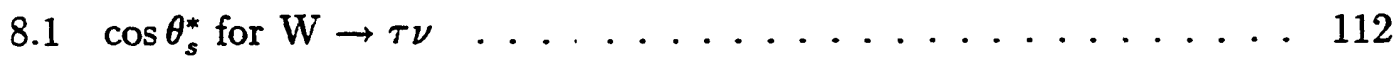

$8.2 \cos \theta_{s}^{*}$ for $\mathrm{Z} \rightarrow \mathrm{e}^{+} \mathrm{e}^{-} \ldots \ldots \ldots \ldots \ldots \ldots \ldots$

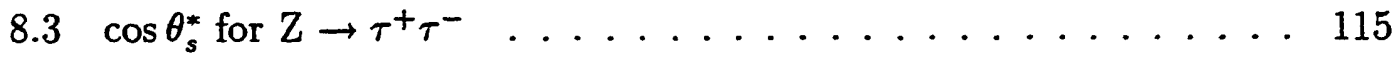

8.4 Transverse Mass Compared to Backgrounds . . . . . . . . . 117

$8.5 \cos \theta_{s}^{*}$ for QCD background . . . . . . . . . . . . 118

$8.6 \cos \theta_{s}^{*}$ for thie Top quark .................. 120

$9.1 \cos \theta_{s}^{*}$ Variations with Absolute Jet Energy Scale . . . . . . . 123

$9.2 \cos \theta_{s}^{*}$ Variations with Absolute Jet Energy Scale . . . . . . . . 124

$9.3 \cos \theta_{s}^{*}$ Variations with Background Contamination . . . . . . . 126

$9.4 \cos \theta_{s}^{*}$ Distribution with and without the Top Quark . . . . . . 127

9.5 Variations in $\cos \theta_{s}^{*}$ Acceptance with PDF . . . . . . . . 129

9.6 Variations in $\cos \theta_{s}^{*}$ Acceptance with Scale . . . . . . . . 130

$9.7 \cos \theta_{s}^{*}$ Distribution for $\mathrm{W}^{+}$and $\mathrm{W}^{-}$Separately . . . . . . . 132

10.1 Final $\cos \theta_{s}^{*}$ Distribution . . . . . . . . . . . . 137

$10.2 \cos \theta_{s}^{*}$ Compared to Spin-1 Theory . . . . . . . . . . . . 138

10.3 Final $\left|\cos \theta_{s}^{*}\right|$ Distribution . . . . . . . . . . . . 139

11.1 Electron Had/EM Efficiency . . . . . . . . . . . . . . . 147

$11.2 \Delta \phi_{\nu J}$ for the 1992 Muon Events . . . . . . . . . . . . . 149

11.3 Selected $M^{*}$ Distribution . . . . . . . . . . . . . 151

11.4 Selected $M^{*}$ Distributions for Each Data Sample . . . . . . . . 152

11.5 Event Display for High-mass Event I . . . . . . . . . . . 153

11.6 Event Display for High-mass Event II . . . . . . . . . . . . . . 154

11.7 Likelihood Function (No Systematics) . . . . . . . . . . . . 156

11.8 Monte Carlo Limits for $500 \mathrm{GeV} q^{*} \ldots$. . . . . . . . . . . . . 159

11.9 Transverse Mass for $q^{*}$ Event Sample . . . . . . . . . . . . . 164

11.10Background $\mathrm{M}_{s}^{*}$ Shape ... . . . . . . . . . . 164

11.11Likelihood Functions Including Systematics . . . . . . . . . . 166 
11.12Limits on $\sigma_{q^{*}} \cdot B_{W J}$ vs Excited Quark Mass $\ldots \ldots \ldots \ldots 168$

11.13Excluded Region in Coupling vs Mass . . . . . . . . . 168 


\section{Chapter 1}

\section{Introduction}

Our understanding of the fundamental nature of the universe has increased immensely in the last century. Special and General Relativity describe space itself and how objects move through it. Quantum mechanics has given us insight into the nature of how objects interact with one another. The Standard Model, although still incomplete, reflects our current knowledge of the objects that exist, or can exist, in the universe, and the forces that govern their interactions.

\subsection{The Standard Model}

The Standard Model is the name given, by High Energy Physics, to a model that describes the known particles and forces. This section describes this model. Tables 1.1 and 1.2 summarize the description, and are a useful reference while reading this section.

The Standard Model represents the results of decades of research by thousands of physicists. The description given here is only very brief. A good historical presentation of the development of the Standard Model is given in [4].

\subsubsection{The Known Forces}

There are three forces known to govern the interaction of particles. The electroweak force ${ }^{1}$, the strong force, and the gravitational force.

Gravity is the weakest of all the forces; therefore, it is the most difficult to study. Little is known about the quantum-mechanical properties of gravity, and the Standard Model includes little more than a recognition of the existence of

\footnotetext{
${ }^{1}$ There are actually two coupling constants associated with the electroweak force; therefore, the Standard Model is sometimes referred to as consisting of four forces.
} 


\begin{tabular}{||c|c|c|c||}
\hline Force & Gauge Boson(s) & Coupling Strength & Boson Mass(GeV) \\
\hline Strong & Gluon & $\alpha_{s} \sim 1$ & 0 \\
Electroweak & Photon & $\alpha \approx 1 / 137$ & 0 \\
& $Z$ & $10^{-5}$ & 91.16 \\
& $W^{ \pm}$ & $10^{-5}$ & 80.0 \\
Gravity & Graviton & $G \approx 10^{-38}$ & $0(?)$ \\
\hline
\end{tabular}

Table 1.1: A summary of the known forces that make up the Standard Model. The couplings shown for the $\mathrm{W}$ and $\mathrm{Z}$ are for low-energy interactions, at high energy they are the same as the photon-1/137.

\begin{tabular}{||ccc|ccl||}
\hline \multicolumn{3}{|c|}{ Leptons } & \multicolumn{3}{c||}{ Quarks } \\
\hline Flavor & Charge $(|e|)$ & Mass $(\mathrm{GeV})$ & Flavor & Charge(|e|) & Mass(GeV) \\
\hline $\mathrm{e}$ & -1 & $5.11 \times 10^{-4}$ & $\mathrm{~d}$ & $-1 / 3$ & 0.01 \\
$\nu_{e}$ & 0 & $<7 \times 10^{-9}$ & $\mathrm{u}$ & $+2 / 3$ & 0.005 \\
\hline$\mu$ & -1 & 0.106 & $\mathrm{~s}$ & $-1 / 3$ & 0.15 \\
$\nu_{\mu}$ & 0 & $<3 \times 10^{-4}$ & $\mathrm{c}$ & $+2 / 3$ & 1.5 \\
\hline$\tau$ & -1 & 1.784 & $\mathrm{~b}$ & $-1 / 3$ & 5.0 \\
$\nu_{\tau}$ & 0 & $<4 \times 10^{-2}$ & $\mathrm{t}$ & $+2 / 3$ & $>113$ \\
\hline
\end{tabular}

Table 1.2: A summary of the known particles that make up the Standard Model. The masses were taken from $[1,2,3]$. The quark masses should be taken as approximate. Since the quarks are always QCD confined, their masses must be inferred from indirect measurements. 
gravity. This is not a problem in current High Energy Physics experiments, because the force is so weak that it does $\mathrm{n}$.st affect the experimental results. The "charge" for the gravitational force (that is the quantity which determines how strongly a given particle will couple to this force) is mass. The gauge boson (an integer-spin particle that mediates a force), which mediates gravity is thought to be spin- 2 and is called the graviton.

The electroweak force was once thought to be two different forces: electromagnetism and the weak force. One of the greatest strides of High Energy Physics in the last two decades was the discovery that these two forces, although vastly different in apparent behavior, are in fact two aspects of the same force. The electroweak force is mediated by four gauge bosons (all spin-1), the photon, $\mathrm{W}^{+}$, $\mathrm{W}^{-}$, and the $\mathrm{Z}$. The $\mathrm{W}$ and $\mathrm{Z}$ bosons, as shown in table 1.1, are very massive, and the photon is massless. In low-energy interactions, the massiveness of the $W$ and $\mathrm{Z}$ bosons causes their interactions to be weak and short ranged. The photon interacts much more strongly, at low energy, and has an infinite range. These differences are why these particles were not previously associated with each other. The "charge" associated with the photon is the electric charge; the $\mathrm{Z}$ boson also couples to electric charge. Particles are also assigned a weak charge which couples to the $\mathrm{W}$ bosons and to the $\mathrm{Z}$ boson.

The strong force is the strongest of the three sorces, and is mediated by the gluon, which is massless and spin-1. The "rinarge" for the strong force is called "color", of which there are three kinds: red, green, and blue. The strong force gauge boson is not only massless, but carries the charge associated with the force (i.e. the gluon is color-charged). This leads to an interesting property of colored objects called confinement. An object that carries a color charge cannot exist on it's own. If a colored object were to exist on its own, the field lines radiating from it would carry an infinite amount of energy (because the field lines are charged). Therefore, objects with color charge are always found bound to other color charged objects, in such a way that the total charge of the combination is zero. The quantum field theory associated with the strong force is known as "Quantum Chromodynamics", or QCD.

\subsubsection{The Known Particles}

The particles in the Standard Model fall into four categories-leptons, quarks, gauge bosons, and the Higgs.

The gauge bosons were discussed in the last section. These particles mediate the fundamental forces, and have integer spin.

The leptons are shown in table 1.2. They consist of the electron, muon, tau, electron neutrino, muon neutrino, and tau neutrino (and their antiparticles). These particles are all spin- $\frac{1}{2}$ and carry electroweak charge, but do not carry color 
charge. Therefore, they do not interact with gluons. Neutrinos only couple to the $\mathrm{W}$ and $\mathrm{Z}$ bosons, and only if they have left-handed helicity (i.e. their spin points opposite to their direction of motion). Antineutrinos only couple if they have right-handed helicity. Therefore, right-handed neutrinos do not exist (this is only strictly true if the neutrinos are massless, which is not yet known). This interesting property of the electroweak force causes parity violating interactions. The tau neutrino has not, yet, been directly observed (there is a particle known to come from tau decays, but it has never been produced in high enough quantities to verify that it is indeed the tau neutrino).

The quarks are also shown in table 1.2. Quarks carry fractional electric charge, and also carry color charge. Since they carry color charge, quarks have never been detected on their own. Quarks are always found in quark-antiquark bound states (known as mesons), or in quark-quark-quark bound states (known as baryons). These combinations of quarks have zero total color charge. As with the leptons, there are three "generations" of quarks: up/down, charm/strange, and top/bottom. The top quark has not, yet, been directly observed. Its mass is believed to be greater than $113 \mathrm{GeV}$, which makes it very difficult to produce this particle.

The final particle in the Standard Model is the Higgs. The Higgs is extremely hypothetical. It has never been observed. The Higgs is an attempt to explain particle masses, and is necessary in order to have the large mass differences between the photon and the $\mathrm{W} / \mathrm{Z}$ bosons. However, the Higgs is by no means the only possibility for explaining these phenomenon. It is, in some sense, the simplest explanation. Often, the Standard Model is referred to as the Minimal Standard Model, because of the inclusion of the minimal Higgs model.

\subsection{This Thesis}

The measurements presented in this thesis contribute two pieces of information to a better understanding of the Standard Model. A sample of events is used in which proton-antiproton collisions produce a $\mathrm{W}$ boson recoiling against a quark or a gluon.

The first measurement presented is the angle of production of the $W$ and quark/gluon. Precise theoretical calculations involving the strong force are difficult to make, because the strong force coupling is so large, and because the nature of the force is quite complicated (it is non-abelian, and has a non-trivial charge structure). This measurement of the scattering angle provides useful information for testing our ability to make predictions in these kinds of events.

The second measurement is of the invariant mass of the $W+$ quark/gluon system. This measurement tests the Standard Model assumption that quarks are 
fundamental particles. It is possible that quarks (and leptons) are actually bound states of some currently unknown particle(s). If quarks are bound states, then it is likely that excited states of the quarks will exist. This measurement searches for these excited quark states, and provides limits on their properties that must be met by any theoretical models for quark compositeness.

This thesis is organized in the following way. Chapter 2 describes the theory of proton-antiproton collisions. Chapter 3 presents an overview of the analysis method used. Chapters 4-9 describe the experimental apparatus and the details of the analysis of the scattering angle. Chapter 10 presents the final scattering angle measurement and compares it with current theoretical predictions. Chapter is describes the search for excited quark states. Concluding remarks are made in Chapter 12. 


\section{Chapter 2}

\section{Theory}

This chapter begins with a description of the theory of proton-antiproton collisions. A discussion then follows of the basic calculational techniques of QCD that are needed for this thesis. Finally $\mathrm{W}$ production, with associated jets, will be discussed.

\subsection{Proton-Antiproton Collisions}

The Fermilab collider provides proton-antiproton collisions with a center-of-mass energy of $1.8 \mathrm{TeV}$ (the proton and antiproton each have momentum $900 \mathrm{GeV}$ ). In collisions such as these, the energy available for producing new particles can be as high as $1.8 \mathrm{TeV}$. The interactions of the fundamental particles can also be studied at very high energies. However, there are some complications associated with using protons. Protons are composite particles; they consist of three valence quarks (uud, and antiprotons are $\bar{u} \bar{u} \bar{d}$ ) as well as gluons and quark-antiquark pairs.

A model has been developed for predicting the behavior of proton collisionsthe parton model. In the parton model, the proton is considered to be a collection of independent quarks and gluons (called partons). When a collision takes place it involves one parton from each of the protons; this interaction is known as the "hard scattering". Figure 2.1 shows a diagram of a generic proton-antiproton collision.

The partons that did not participate in the hard scattering are referred to as the "spectator partons". The interactions among the spectator partons are relatively low energy; therefore, it is difficult to calculate their behavior (this will be explained more in section 2.3, where perturbation theory is discussed). The spectator parton interactions typically produce a large number of low-energy hadrons that are uniformly distributed over the detector. This energy deposition 


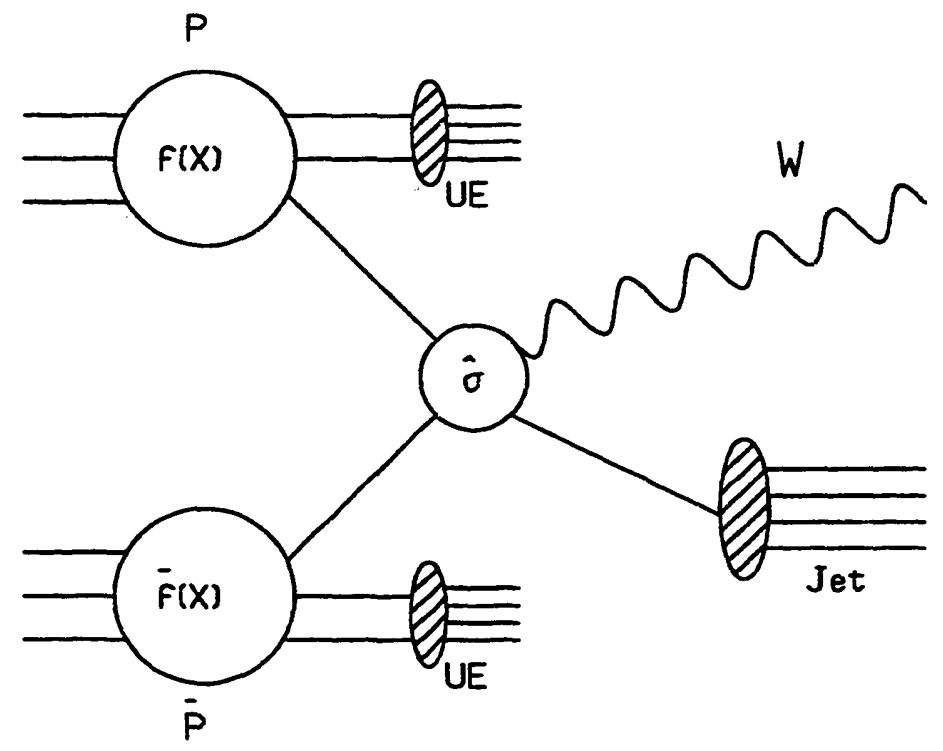

Figure 2.1: A schematic diagram of a hadronic collision, showing the partons from the incoming nucleons participating in the hard scattering. The remaining partons form the underlying event (UE). In this example a $W$ boson and a Jet are produced in the hard scattering. 
will be referred to as the "underlying event". The underlying event does not, typically, interfere significantly with measurements made using the hard scattering system.

The momentum of the partons that produce the hard scattering can vary from nearly zero to nearly the full momenturn of the proton. The hard scattering system, therefore, is not usually at rest in the lab frame. In fact it can have large boosts (i.e. Lorentz transformations with large $\gamma \beta$ ) along the beam directions in the lab frame. The center-of-mass energy of the hard scattering system can, therefore, also vary from nearly zero to nearly the energy of the proton-antiproton system.

The momentum distribution of the partons is predicted using proton "parton distribution funciions" (pdf's). Thesf functions represent the probability that a given type of parton (gluon, up quark, down quark, etc.) will carry a given fraction $(x)$ of the proton's momentum. Calculating the cross section for protonantiproton collisions resulting in a given final state $(X)$ involves convoluting the pdf's with the cross section for the hard scattering (partons $\rightarrow X$ ). That is, the cross section for proton-antiproton going to $X, \sigma(p \vec{p} \rightarrow X)$ is:

$$
\sigma(p \bar{p} \rightarrow X)=\sum_{i j} \int_{0}^{1} \int_{0}^{1} d x_{i} d x_{j} f\left(x_{i}\right) \bar{f}\left(x_{j}\right) \hat{\sigma}\left(p_{i} p_{j} \rightarrow X\right)
$$

where the indices $i$ and $j$ refer to the type of parton (up, down, charm, strange, top, bottom, or gluon). The functions $f$ and $\bar{f}$ are the parton distribution functions. $f\left(x_{i}\right)$ is the probability density for a parton of type $i$ to be found in the proton with momentum $x_{i}$ times the momentum of the proton $\left(0<x_{i}<1\right) . \bar{f}\left(x_{j}\right)$ is the corresponding structure function for the antiproton. $\hat{\sigma}\left(p_{i} p_{j} \rightarrow X\right)$ is the cross section for partons of type $i$ and $j$, with momentum fractions $x_{i}$ and $x_{j}$, to produce the final state $X$. Figure 2.2 shows a typical set of parton distribution functions.

The parton distribution functions are measured in experiments in which high energy leptons (electrons and neutrinos) are used to probe the structure of the proton[5]. It is difficult to measure the gluon content of the proton using these types of experiments; therefore theoretical models must be used to extrapolate the gluon fractions in some $x$ ranges. Using different models for this extrapolation (and to some extent using different types of experimental data) results in slightly different sets of parton distribution functions. This uncertainty usually leads to small uncertainties in measurements made using proton-antiproton collisions.

\subsection{Coordinate System and Units}

In this section the coordinate system used to describe the proton-antiproton collisions is described. The protons and antiprotons collide, from opposite directions, 


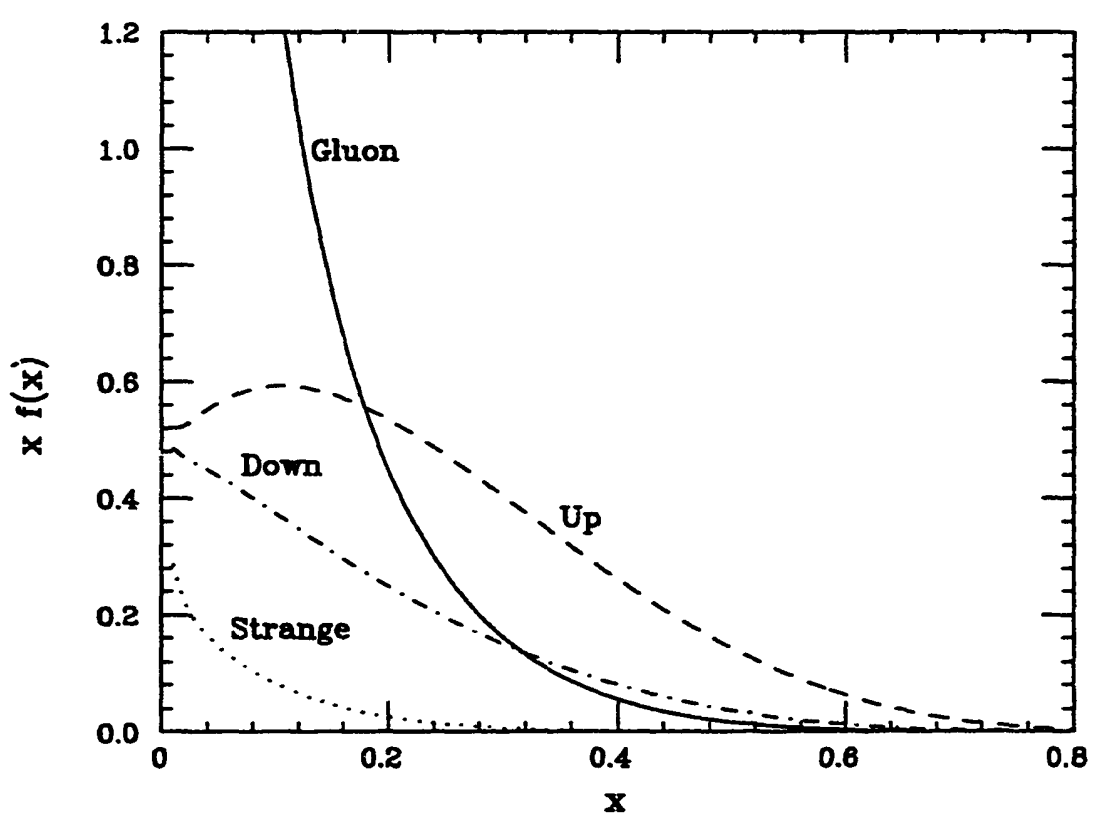

Figure 2.2: HMRSB parton distribution functions as a function of $x$ (the fraction of the proton's momentum carried by the parton). The quantity plotted is $x \cdot f(x)$, which is the pdf weighted by the momentum fraction itself. The plot shows the up quark, down quark, strange quark, and gluon components of the proton. 
along a single line. This line is taken as the $\mathrm{z}$-axis. The protons are taken as traveling in the positive $z$-direction, and $z=0$ is taken to be the center of the CDF detector. A standard spherical coordinate system is then used. $\theta$ is the polar angle from the positive $z$-axis, and $\phi$ is the azimuthal angle around the $z$-axis $(\phi=0$ is defined with respect to the CDF detector, and is irrelevant for this analysis).

Since the hard scattering system typically has very large boosts along the $z$ direction, the particles produced in the collisions are not distributed uniformly in $\cos (\theta)$. Instead, they tend to be uniformly distributed in $\eta$ (pseudo-rapidity), where

$$
\eta=-\ln \left(\tan \frac{\theta}{2}\right) .
$$

Another concept that will be used throughout this thesis is that of "transverse momentum", $\mathrm{P}_{t}$. This simply refers to the components of the momentum in the $x-y$ plane. Transverse momentum is invariant under boosts (of the hard scattering system) along the $z$ direction; therefore, it is a very useful quantity in protonantiproton collisions.

\section{Units}

Energy, momentum, and mass will all be referred to in this thesis in units of $\mathrm{eV}$ (electron volts). This is a widely used convention in which the speed of light, $c$, is taken to be identically equal to 1 . This system is used for its notational simplicity (i.e. momentum does not have to be given in units of $\mathrm{eV} / c$, and mass in units of $\left.\mathrm{eV} / \mathrm{c}^{2}\right)$.

\subsection{QCD Perturbation Theory}

Perturbation theory is used, with very few excepuions, for calculating the hard scattering cross sections. The following sections describe some of the issues that arise as the result of using perturbation theory to predict high-energy hard scatterings.

\subsubsection{Momentum Scale}

Figure 2.3 shows the leading order Feynman diagrams for the scattering of two partons into a $\mathrm{W}$ and a parton. These diagrams are first order in the electroweak coupling, $\alpha$, and first order in the strong coupling, $\alpha_{s}$. Since gluons carry color charge, they can couple to each other. This greatly increases the difficulty in carrying out perturbation theory calculations higher than leading order in $\alpha_{s}$. 
Periurbation theory calculations carried out only to leading order contain a parameter, $Q^{2}$, which represents the momentum scale at which the coupling constant should be evaluated. $Q^{2}$ is essentially arbitrary; however, it only enters the cross sections as a factor of $\ln \left(Q^{2}\right)$. The $Q^{2}$ scale is usually taken as some typical momentum transfer in the event. In the $W+$ parton case it is usually taken as the transverse momentum of the $\mathrm{W}$. As an estimate of the uncertainty due to this approximation, the $Q^{2}$ scale is usually varied by a factor of 4 . The total cross section can vary significantly when $Q^{2}$ is varied, but the kinematic properties of the events (e.g. the scattering angle distribution) are much less sensitive to the choice of scale.

Parton distribution functions also depend on the $Q^{2}$ scale. They are measured in experiments where $Q^{2}$ is relatively small compared to the $Q^{2}$ scales at the Fermilab collider. Therefore, they must be "evolved" up to high $Q^{2}$ values using the Altarelli-Parisi equations[6]. The same $Q^{2}$ scale is used for evaluating the parton distribution functions that is used to evaluate the hard scattering cross section. For a leading order hard scattering prediction, leading order pdf's should be used. For a next-to-leading order hard scattering prediction, next-to-leading order pdf's should be used. In this way, the calculations used to evolve the pdf's will not contain diagrams that are already included in the hard scattering calculation.

\subsubsection{Singularities}

Leading order calculations are also plagued by divergencies; however, these can be dealt with. If any two parton lines are parallel, or if any of the partons is too low in energy, the cross section becomes infinite. These divergencies ("collinear" and "infrared") would not exist if higher order diagrams were included. As long as the calculations only involve regions of phase space in which lines are not too parallel or too low-energy, then the predictions are expected to be reasonable. The cuts made in the theoretical calculations are often chosen to match cuts that can be made in the experimental situations. If the partons are required to have a reasonably large transverse momentum, then the infrared divergence is avoided. A transverse momentum cut is needed in experimental measurements, anyway, because detecting low transverse momentum partons is very difficult (discussed in section 2.3.3). The collinear divergence is avoided by requiring the partons to be separated from one another in $\eta-\phi$ space. This is also needed in experimental situations, because partons tirat are too close together will not be distinguishable. 


\subsubsection{Parton Fragmentation}

Color confinement prevents quarks and gluons from existing on their own for more than a very short time. As the partons travel away from the hard scattering, they will be acted on by strong attractive QCD forces. Quark-antiquark pairs will be created from the color force lines joining all the partons in the event. The result is that a quark/gluon, produced in the hard scattering, will result in a collimated "jet" of hadrons and baryons exiting from the collision. Throughout this thesis, the word "jet" will be used to generically refer to quarks or gluons.

This process, known as "fragmentation", involves very low momentum transfers. The coupling constant in QCD is very large for low momentum transfers (approximately 1), which makes perturbation theory u eless for describing fragmentation. Empirical models must be used to predict the properties of jets. As long as the jets can be required to be well separated from one another, and from any other objects in an event, uncertainties in the details of the fragmentation process will not affect experimental results significantly. In this thesis, the jets are required to be well separated from other particles in the event, so the analysis is insensitive to the details of jet fragmentation.

\subsubsection{Intrinsic $\mathrm{K}_{t}$}

The diagrams in figure 2.3 represent events in which the $W+$ jet system has no net transverse momentum. In a real event, the hard scattering system would have many low-energy gluons emitted from the quark and gluon lines, which would give a small transverse momentum to the $\mathrm{W}+$ jet system. This transverse momentum will also be referred to as a " $\mathrm{K}_{t}$ kick". It is very difficult to theoretically predict the $K_{t}$ kick. What is usually used is an empirical model based on the $K_{t}$ kick measured in the collisions. The $\mathrm{K}_{t}$ has little effect on the analysis in this thesis.

\subsection{W Production at Hadron Colliders}

In this thesis, the properties of $\mathrm{W}+$ jet events will be studied. Figure 2.3 shows the leading order (LO) Feynman diagrams for the production of $\mathrm{W}+$ jet events. As examples, the two most likely reactions are $\mathrm{d} \overline{\mathrm{u}} \rightarrow \mathrm{gW}^{-}$and $\mathrm{g} \overline{\mathrm{u}} \rightarrow \overline{\mathrm{d}} \mathrm{W}^{-}$ (similarly for the $\mathrm{W}^{+}$boson).

These diagrams involve a spin- $\frac{1}{2}$ propagator (a quark), and so the production angle of the $\mathrm{W}$ boson will be much less forward peaked than a Rutherford-type scattering involving a spin-1 photon or gluon (e.g. $q \bar{q} \rightarrow q \bar{q}$ or $g g \rightarrow g g$ ). Rutherford scattering has a $\sin (\theta / 2)^{-4}$ dependence at large production angles, while a spin- $\frac{1}{2}$ propagator has a $\sin (\theta / 2)^{-2}$ dependence. 

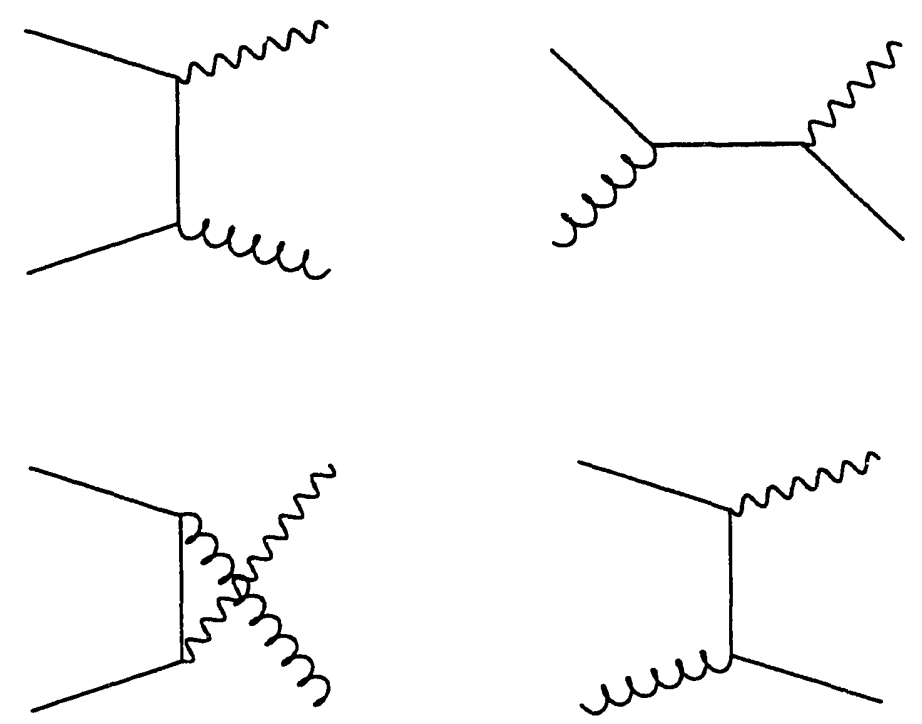

Figure 2.3: The leading order Feynman diagrams for $\mathrm{W}+$ jet production. 


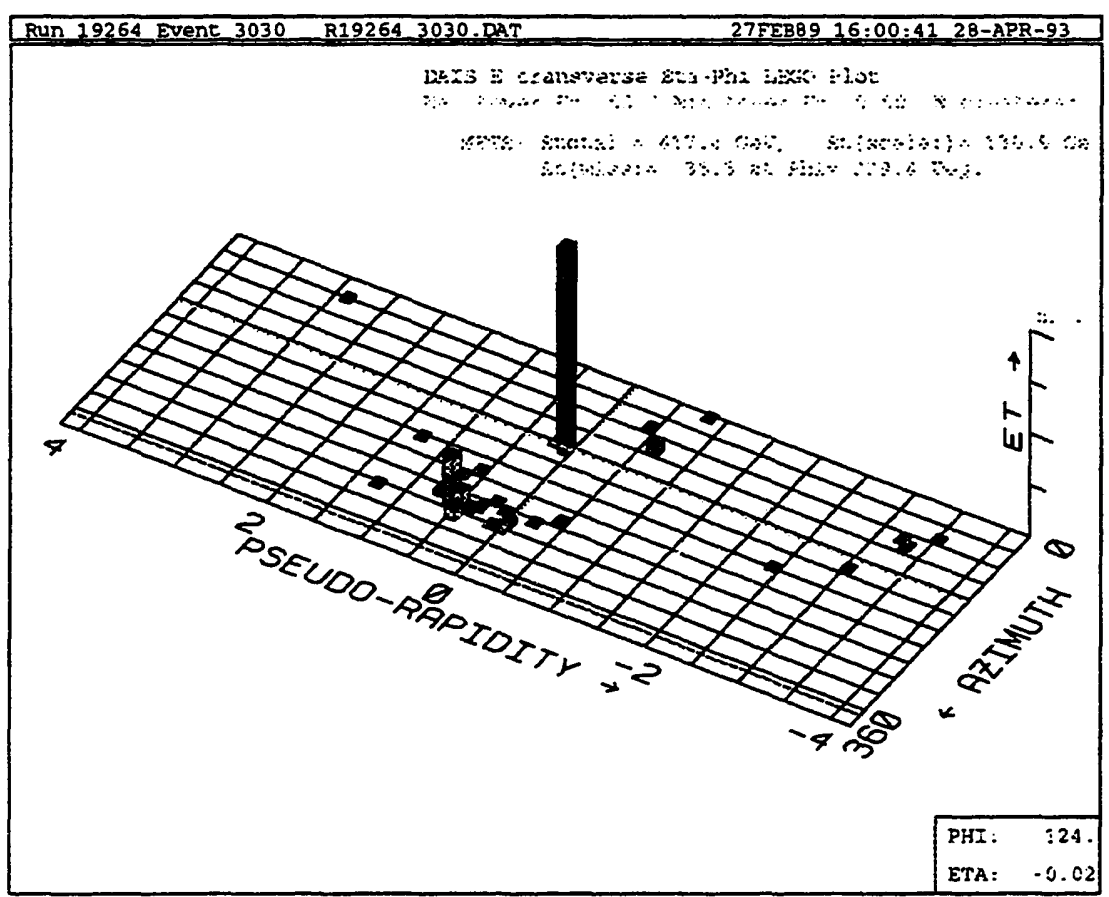

Figure 2.4: The CDF calorimeter energy deposition for a typical $W+$ jet event. The calorimeter has been "unrolled" to make this lego plot. Energy in the electromagnetic calorimeters is shown darker than the hadronic energy. The tower heights are proportional to the transverse energy in each tower. The tracking information for this particular event is shown in figure 4.6.

Approximately $25 \%$ of the $\mathrm{W}+$ jet events will contain more than one jet. Roughly speaking, every additional jet in an event adds of factor of $\alpha_{s}$ to the cross section calculation. At the energies occurring in the events used in this analysis, $\alpha_{s}$ is typically $\leq 0.2$. The data shows that approximately $1 / 5$ of the events contain a second jet, and $1 / 5$ of those contain a third jet, etc. In this thesis, the highest $\mathrm{P}_{t}$ jet will be grouped with the $\mathrm{W}$ to form the $\mathrm{W}+$ jet system. This is essentially a convention for tivacing events that have multiple jets in them. Since the leading order theory diverges when jets become collinear, it is difficult to make predictions for events with multiple jets. The effects of the other jets in the event will be shown to be small.

Figure 2.4 shows a display of a typical $\mathrm{W}+$ Jet event produced in the Fermilab Tevatron and recorded by the CDF detector. The electron and the jet are clearly seen as large clusters of energy in the calorimeters. The underlying event, and radiated low- $\mathrm{P}_{t}$ gluons manifest themselves as the small number of low-energy towers that are scattered about the detector.

Chapter 3 continues the discussion of the properties of $W+$ jet events produced in $\mathrm{p} \overline{\mathrm{p}}$ collisions. 


\section{Chapter 3}

\section{Analysis Overview}

This chapter is an overview of the analysis method used in this thesis. Without getting "bogged down" in the details of the experiment, it describes the philosophy and the underlying theory of the analysis.

For the purpose of illustration, in this section, a simple Monte Carlo program is used to simulate $p \bar{p} \rightarrow W+$ jet events. The program, called VECBOS [7], is based on a tree-level calculation of the Feynman diagrams shown in figure 2.3. For the purposes of this section VECBOS should be considered a "black-box" program that produces events with a jet (either a quark or a gluon) and a $\mathrm{W}$ boson-which is decayed to an electron and a neutrino. It will be shown later in this thesis that the properties of these Monte Carlo events closely approximate those of real events recorded by $\mathrm{CDF}$, and therefore this is an adequate model for illustrating the analysis method.

\subsection{Properties of $\mathbf{W}+$ Jet Events}

This section describes the properties of the Monte Carlo W + Jet events. Assume in this section that the momenta of all the particles are known-recall that the outgoing particles are the electron, neutrino, and the jet. The calculation is only leading order therefore all of the exiting particles are zero mass (a good approximation given the high momentum of the particles).

\subsubsection{Collins-Soper Reference Frame}

Given that the 4-momenta of the particles are known, one can boost from the lab frame into the $\mathrm{W}+$ jet center-of-mass frame, which is shown in figure 3.1. In the figure, $P_{p}^{\prime}$ is the 4-vector that results from boosting a massless particle which, in the lab frame, is travelling in the proton direction into the $\mathrm{W}+$ Jet center-of-mass 
frame. In the same way, $P_{\bar{p}}^{\prime}$ represents the antiproton's direction. The z-axis in this frame is then defined such that the positive $z$-axis bisects the angle between $P_{p}^{\prime}$ and $-P_{\bar{p}}^{\prime}$. This definition of the z-axis was first proposed by Collins and Soper [8]; therefore, this frame is usually referred to as the "Collins-Soper frame" or the "CS frame".

In the simple VECBOS Monte Carlo, the hard-scattering system will not have any net transverse momentum (i.e. the boost into the center-of-mass system will be entirely along the $z$-axis). However, in a real collision (or in an extended version of VECBOS to be described later) there may be a small transverse component. The CS frame was first introduced[8] in order to minimize the effects of this transverse momentum. It also serves as a "conventional" frame in which most experimental measurements and most theoretical calculations are presented.

A convenient method of boosting into the CS frame is to do the boost in two steps. First, the lab particles are boosted purely along the $z$-direction so that the $z$-momentum of the hard scattering system is zero. Then the particles are boosted perpendicular to the $z$-axis into the center-of-mass frame. Boosting in this way will result in a frame in which the CS z-axis is parallel to the lab zaxis (a convenient property when one is dealing with these frames in a computer program). This procedure will be referred to as boosting using the "Collins-Soper prescription".

\subsubsection{Center-of-mass Variables}

Figure 3.2 illustrates the definition of $\cos \theta^{*}$, which is the cosine of the angle between the $\mathrm{W}^{-}$and the positive $\mathrm{z}$-axis. In $\mathrm{W}^{+}$events the sign of the z-component of the momentum of each particle is inverted and the event is then treated as if it were a $\mathrm{W}^{-}$(this is equivalent to inverting $\cos \theta^{*}$ for $\mathrm{W}^{+}$'s). This treatment of the $\mathrm{W}^{+}$events preserves any asymmetries that would otherwise be hidden. These events should be CP invariant; therefore, the inverted $\mathrm{W}^{+}$distributions should be identical to the $\mathrm{W}^{-}$distributions.

This analysis is an attempt to measure a variable (to be defined later) whose distribution is sensitive to the $\cos \theta^{*}$ distribution. One cannot measure $\cos \theta^{*}$ directly because contrary to the assumption of this section, the neutrino's momentum is not directly measurable. This problem will be discussed in the next section. This section will continue to assume that $\cos \theta^{*}$ is measurable in order to illustrate the properties of $\mathrm{W}+$ jet events.

Before plotting the distribution of $\cos \theta^{*}$, one cut needs to be applied to these events. The leading order diagrams for this process diverge when the transverse momentum $\left(\mathrm{P}_{t}\right)$ of the jet is small; therefore, a cut was made to require the $\mathrm{P}_{t}$ to be greater than $15 \mathrm{GeV}^{1}$. The $\mathrm{P}_{t}$ cut on the jet will distort the distribution

\footnotetext{
${ }^{1}$ This requirement on the jet is also needed in the experiment, because jets with less than 15
} 


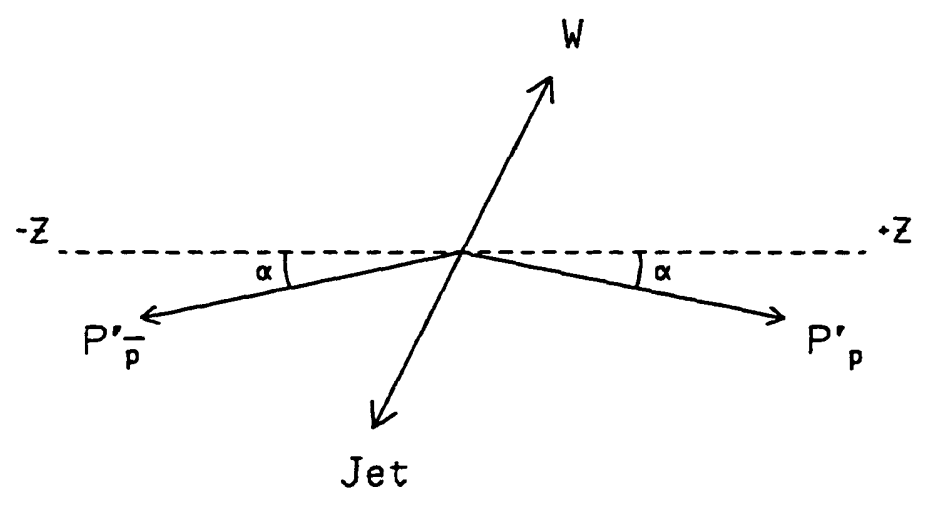

Figure 3.1: The Collins-Soper reference frame. The $z$ axis is defined such that the two angles shown as $\alpha$ are equal. $P_{p}^{\prime}$ and $P_{\bar{p}}^{\prime}$ represent the proton and antiproton massless momenta (described in the text).

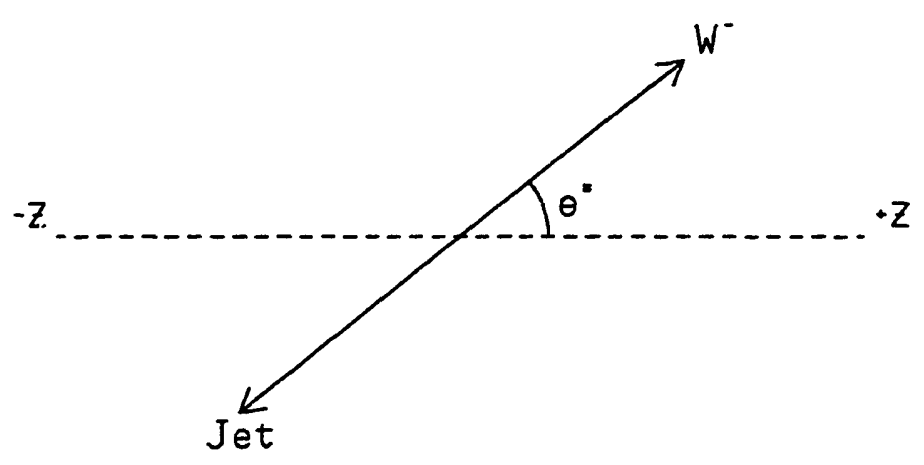

Figure 3.2: The $W+$ Jet center-of-mass frame. The Collins-Soper definition of the $z$-axis is used. 
of $\cos \theta^{*}$, by preferentially cutting out events with large $\left|\cos \theta^{*}\right|$. This acceptance effect can be removed by placing a cut on the range of $\cos \theta^{*}$ to be studied and a cut on the total energy $\left(\mathrm{M}^{*}\right)$ in the center-of-mass frame. For this analysis $\cos \theta^{*}$ is required to be in the range $-0.9<\cos \theta^{*}<0.9$. The $\mathrm{M}^{*}$ cut needed is then determined by the extreme case of an event with $P_{t}$ at the cutoff and $\cos \theta^{*}$ at the cutoff; in this configuration the total energy is

$$
\mathrm{M}_{\text {cut }}^{*}=\frac{P_{t}^{\text {cut }}}{\sin \theta_{\text {cut }}^{*}}+\sqrt{\left[\frac{P_{t}^{\text {cut }}}{\sin \theta_{c u t}^{*}}\right]^{2}+M_{w}^{2}} .
$$

With a $15 \mathrm{GeV} \mathrm{P}_{t}$ cut and the 0.9 cut on $\left|\cos \theta^{*}\right|$ the requirement on $\mathrm{M}^{*}$ is $M^{*}>121.5 \mathrm{GeV}$. With this $\mathrm{M}^{*}$ cut, no combination of $\mathrm{M}^{*}$ and $\cos \theta^{*}$ can result in a jet with $\mathrm{P}_{t}$ less than $15.0 \mathrm{GeV}$. Figure 3.3 shows the $\cos \theta^{*}$ distribution with these cuts applied.

The $\cos \theta^{*}$ distribution shown in figure 3.3 is slightly asymmetric (as described in the figure caption, the sign of $\cos \theta^{*}$ is defined differently for $\mathrm{W}^{+}$and $\mathrm{W}^{-}$, so this asymmetry does not demonstrate $\mathrm{CP}$ violation in the Monte Carlo program). This asymmetry is caused by events in which the initial state is a collision between a quark and a gluon $(q+g \rightarrow W+q)$. In these events, the $W$ tends to be produced in the direction of the incoming quark. Consider the $\mathrm{W}^{-}$(the same argument applies to $\mathrm{W}^{+}$, with different quark flavors), it can be produced by a $d$ quark from the proton (and a gluon from the antiproton) or by a $\overline{\mathrm{u}}$ quark from the antiproton. If these two contributions were equal, then there would be no asymmetry in $\cos \theta^{*}$; however, it is known that the $u$ quarks tend to carry more of the proton momesitum than do the $\mathrm{d}$ quarks (so the $\mathrm{u}$ quarks contribute more to $\mathrm{W}$ production). Since the $\overline{\mathrm{u}}$ process dominates, the $\mathrm{W}^{-}$tends to be produced more in the negative- $z$ direction (the asymmetry seen in figure 3.3). Unfortunately, this asymmetry is too small to measure using the $\cos \theta^{*}$ distribution (using the data currently recorded by CDF).

Figure 3.4 shows the distribution of $\mathrm{M}^{*}$. The cross section falls off rapidly with mass; however, it does not "blow up" at small values because it must turn over and go to zero at roughly the mass of the $\mathrm{W}$ boson $(80 \mathrm{GeV})$.

Figure 3.5 shows the distribution of $Y_{\text {Boost }}$ (the rapidity of the boost, along the $\mathrm{z}$-axis, into the $\mathrm{W}+$ Jet COM frame). The distribution, like $\cos \theta^{*}$, is asymmetric due to the fact that the up-quarks tend to carry more of the proton momentum than do the $d$ quarks.

\subsubsection{W Rest Framie}

Another interesting property of the $W$ events is the polarization state of the $W$. This can be studied by boosting into the rest frame of the $W$, using the Collins

$\mathrm{GeV}$ transverse momentum are difficult to measure. 


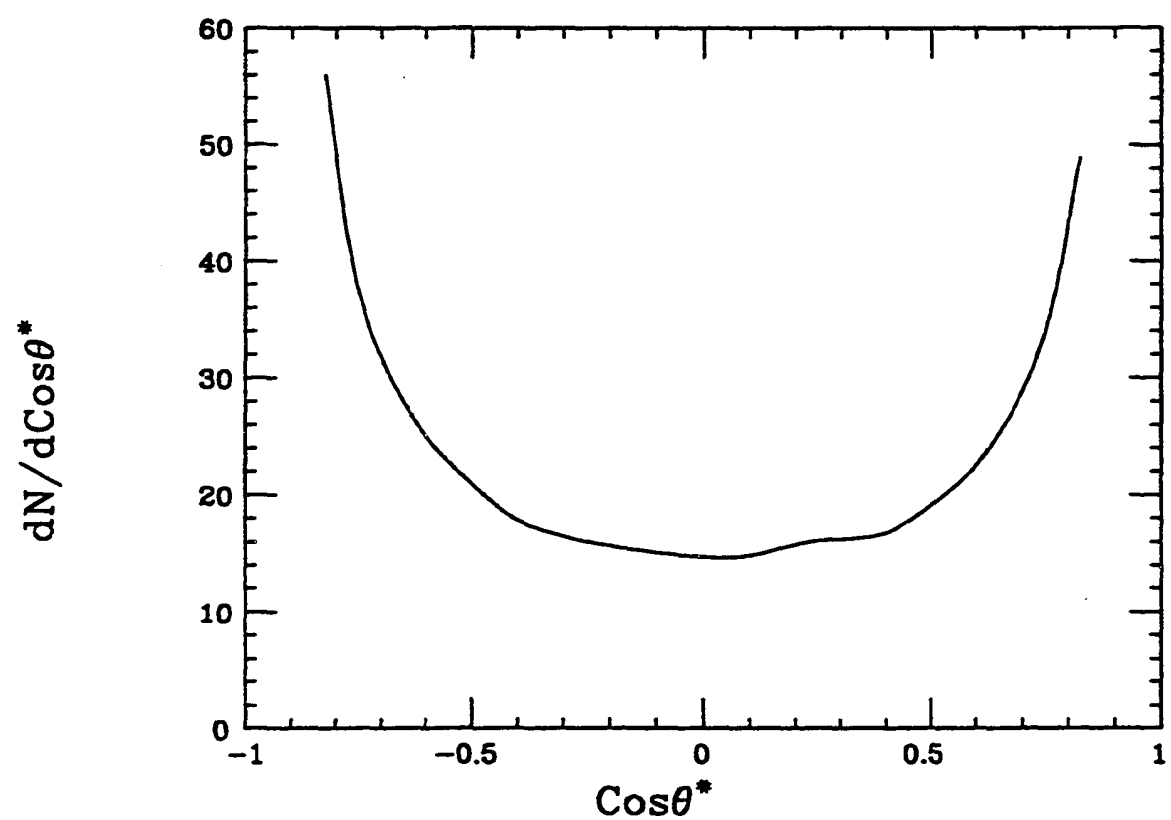

Figure 3.3: $\cos \theta^{*}$ distribution for the vecBos Monte Carlo sample, where $\cos \theta^{*}$ is the angle between the $\mathrm{W}^{-}\left(\mathrm{W}^{+}\right)$and the $z^{+}\left(z^{-}\right)$direction. HMRSB parton distribution functions were used with the scale set to $1 / 2$ of the jet's $P_{t}$. 


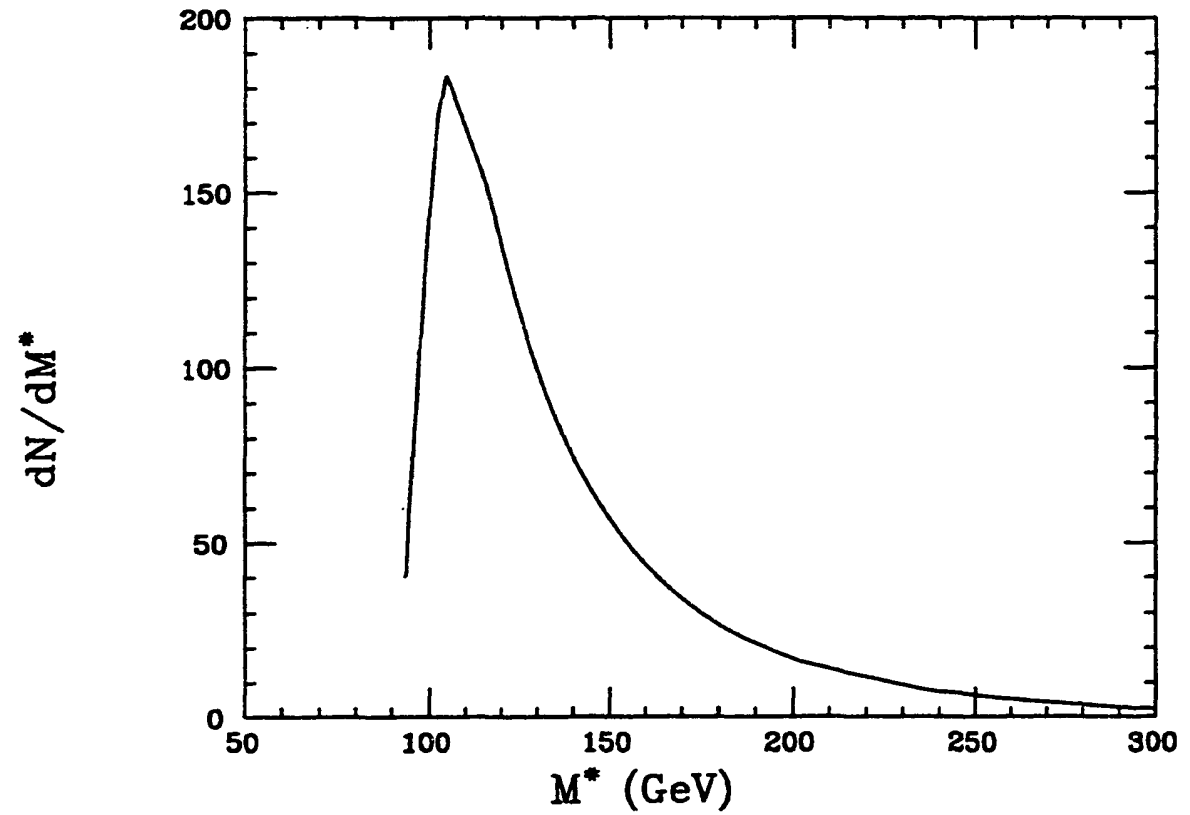

Figure 3.4: $M^{*}$ distribution for the vecbos Monte Carlo sample, where $M^{*}$ is the total energy in the center-of-mass frame. HMRSB parton distribution functions were used with the scale set to $1 / 2$ of the jet's $P_{t}$. 


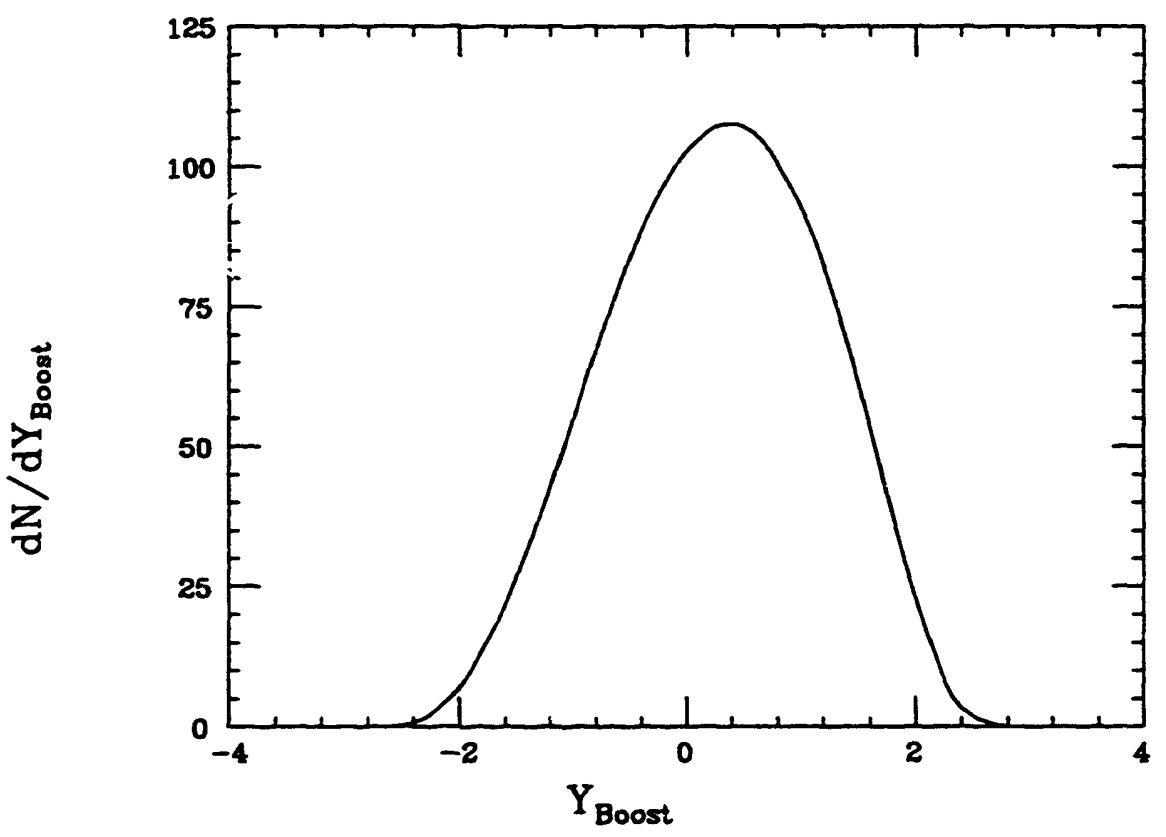

Figure 3.5: $Y_{\text {Boost }}$ distribution for the vecBos Monte Carlo sample, where $Y_{\text {Boost }}$ is the change in rapidity in going from the lab frame to the center-of-mass frame. HMRSB parton distribution functions were used with the scale set to $1 / 2$ of the jet's $P_{t}$. 
and Soper prescription. W production is dominated by initial states that involve at least one valence quark. Any initial state involving a valence quark will produce a $\mathrm{W}^{-}$which tends to be polarized along the negative $\mathrm{z}$ direction, while the $\mathrm{W}^{+} \dot{\mathrm{s}}$ will tend to be polarized along the positive $\mathrm{z}$ direction. The $\mathrm{W}^{-}$only couples to negative helicity electrons, therefore the polarization of the $W$ will result in an asymmetric decay distribution. Figure 3.6 shows the $W$ rest frame and defines the angle $\alpha_{C S}$ as the angle between the electron and the positive z-axis. Figure 3.7 shows the distribution of $\cos \alpha_{C S}$. The polarization of the $\mathrm{W}$ causes the $\cos \alpha_{C S}$ distribution to be very asymmetric. If the $W$ were polarized completely along the negative $\mathrm{z}$ axis, $\cos \alpha_{C S}$ would have the distribution:

$$
\frac{d \sigma}{d \cos \alpha_{C S}} \propto\left(1+\cos \alpha_{C S}\right)^{2} \text {. }
$$

The actual distribution differs from this for two reasons. The sea-quark component of the nucleons can produce $\mathrm{W}^{-}$polarized in the positive $z$ direction. The other reason is that the W's are produced with another particle (sometimes a quark and sometimes a gluon) and, therefore, the polarization of the $W$ is not exactly along the $\mathrm{z}$ direction.

The other variable describing the decay of the $W$ is the azimuthal angle, around the z-axis, of the electron: $\phi_{C S}$. The angle $\phi_{C S}$ is defined to be zero in the plane of $P^{\prime}{ }_{p}$ and $P_{\bar{p}}^{\prime}$ and on the opposite side of these two vectors (i.e. $\phi_{C S}$ is zero in the direction of the lab W momentum). Figure 3.8 shows the distribution of $\phi_{C S}$. The distribution is quite flat, which indicates that the $W$ polarization does tend to be along the z-axis.

\subsection{The Undetected Neutrino}

The biggest complication for this analysis is the fact that the neutrino interacts so weakly that it passes through the CDF detector leaving no trace of its passage.

This section describes how the neutrino momentum can be almost fully reconstructed from other information available in the event. It also discusses the problems that arise due to the lack of information about the neutrino momentum, and the method used to deal with these problems.

\subsubsection{Determination of the Neutrino Momentum}

Most of the particles that are produced in the proton-antiproton collision reach the CDF detector. Only those particles that exit very close to the beamline $(|\eta|>3.6)$ will be missed. The missed particles, therefore, will not carry away much transverse momentum. So, the $\mathrm{x}$ and $\mathrm{y}$ components of the neutrino's momentum can be determined by summing the $x$ and $y$ components of all the particles 


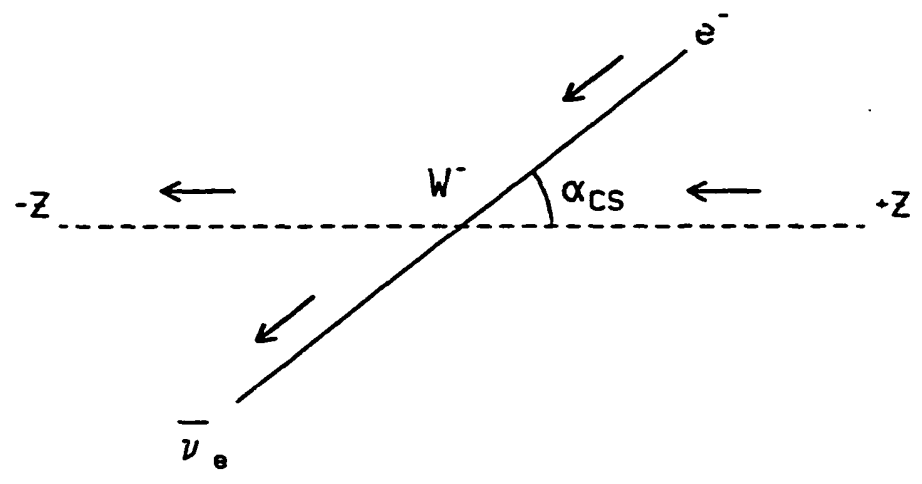

Figure 3.6: The Collins-Soper rest frame of the $\mathrm{W}$ boson. The angle $\alpha_{C S}$ is the angle between the outgoing electron(positron) and the positive(negative) $\mathrm{z}$-axis. The arrows show the helicity states of the incoming and outgoing particles in a typical event.

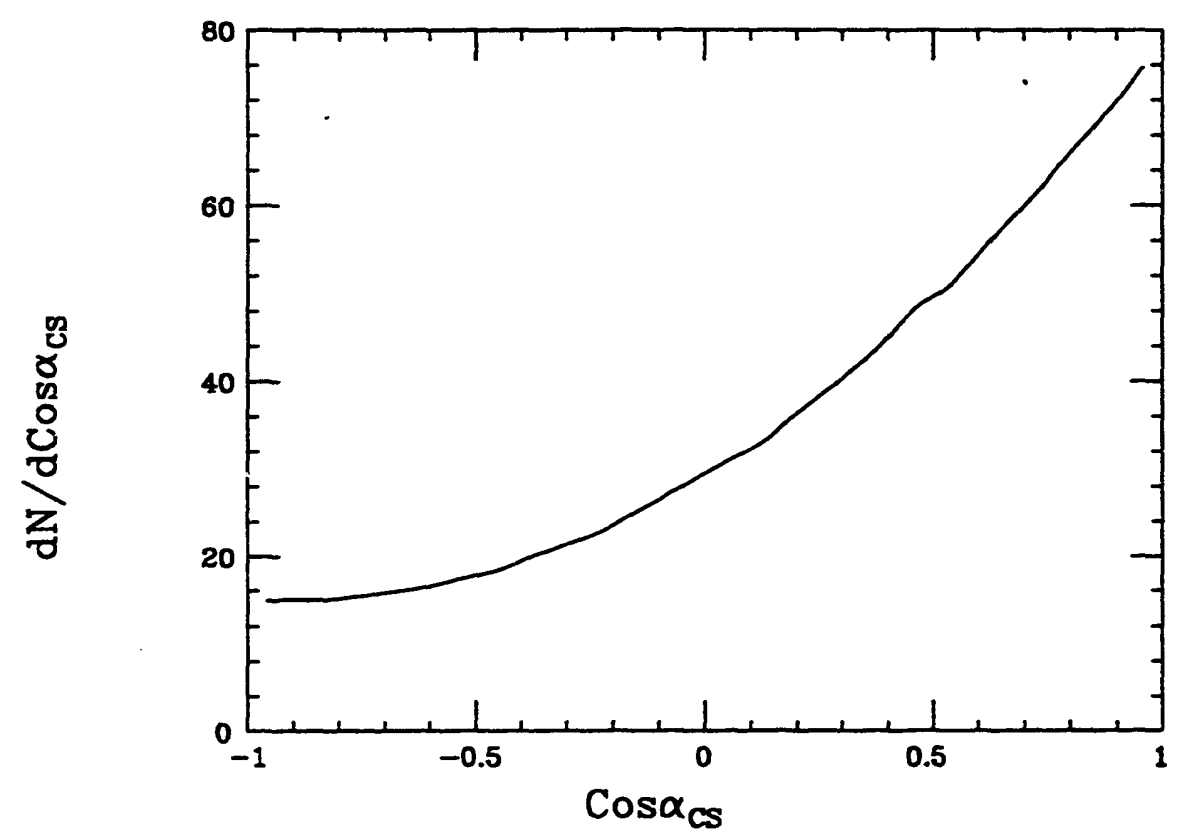

Figure 3.7: The distribution of $\cos \alpha_{C S}$ for the VECBOS parton model. HMRSB parton distribution functions were used with the scale set to $1 / 2$ of the jet's $P_{t}$. 


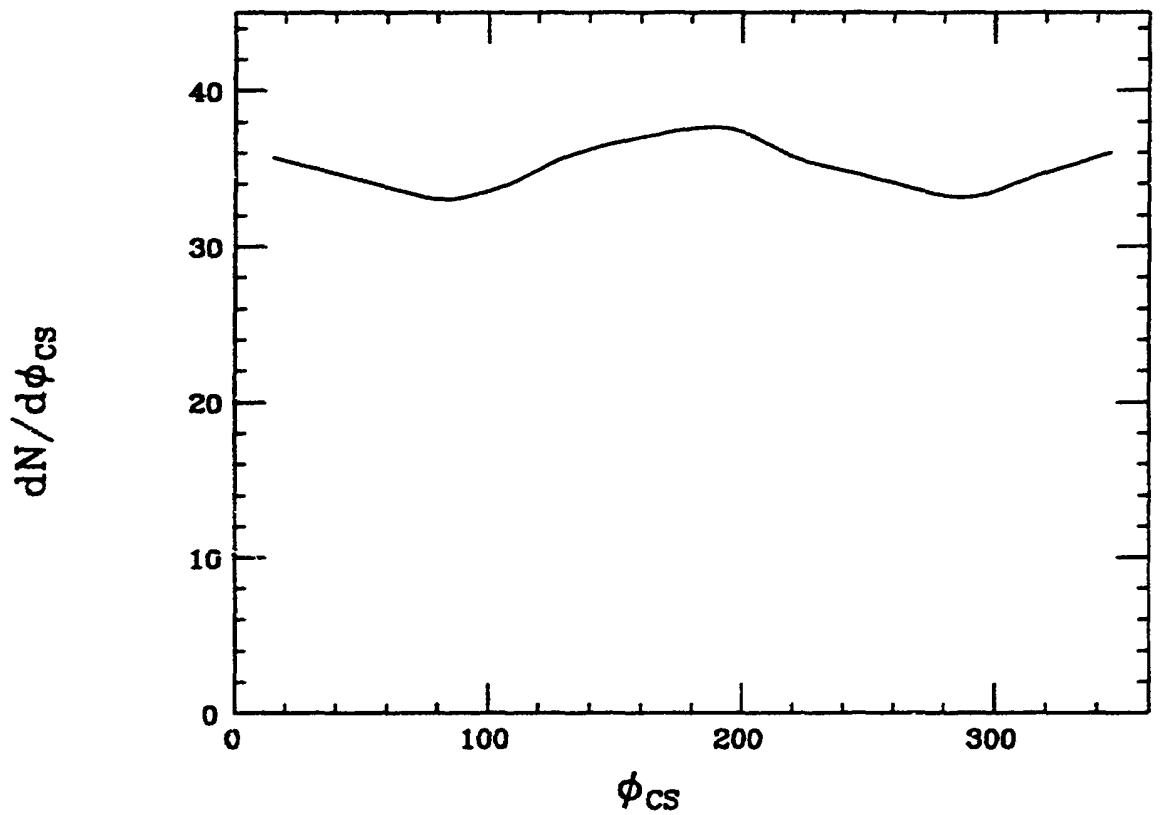

Figure 3.8: The distribution of $\phi_{C S}$ for the VECBOS parton model. HMRSB parton distribution functions were used with the scale set to $1 / 2$ of the jet's $P_{t}$. 
measured in the detector. Any imbalance in transverse momentum is assuciated with the neutrino.

This method cannot be used to determine the z-component of the neutrino's momentum $\left(\mathrm{P}_{z}^{\nu}\right)$. The hard scattering systeni can have a significant boost along the $z$ direction (which varies greatly from event to event). Unfortunately, particles exiting very close to the beamline can carry away large amounts of $z$-momentum, so $\mathrm{P}_{z}^{\nu}$ cannot be determined from the measured information.

If one assumes, however, that the neutrino and the electron are the decay products of a $\mathrm{W}$ boson, then $\mathrm{P}_{z}^{\nu}$ can be constrained. If the invariant-mass of the electron-plus-neutrino system is required to be the mass of the $\mathrm{W}\left(\mathrm{M}_{W}\right), \mathrm{P}_{z}^{\nu}$ is constrained to two values:

$$
\mathrm{P}_{z}^{\nu}=\frac{1}{2 P_{t e}^{2}}\left[A P_{z e} \pm E_{e} \sqrt{A^{2}-4 P_{t e}^{2} P_{t \nu}^{2}}\right]
$$

where

$$
A=1+P_{t W}^{2}-P_{t e}^{2}-P_{t \nu}^{2} \text {. }
$$

The transverse momenta of the W, electron, and neutrino are $P_{t W}, P_{t e}$, and $P_{t \nu}$ respectively, and $P_{z e}$ and $E_{e}$ are the electron $z$ momentum and energy.

Some events will produce imaginary (or complex) solutions for $\mathrm{P}_{z}^{\nu}$; section 7.1.1 describes the treatment of these constraint failures. Another small complication is that the $\mathrm{W}$ has a natural width, so the constraint cannot be made to the actual $W$ mass in each event, but only to the average value of the mass $-80 \mathrm{GeV}$. This produces a smearing in the final distribution, but the smearing is small and has a negligible effect (this is discussed in more detail in section 7.8).

\subsubsection{Selection of One Neutrino Solution}

The two-fold ambiguity in $\mathrm{P}_{z}^{\nu}$ causes a two-fold ambiguity in the variables $\cos \theta^{*}$, $\mathrm{M}^{*}, Y_{\text {Boost }}$, and $\cos \alpha_{C S}$ (all described in the previous section). One of the solutions is the "true" event solution (corresponding the real $\mathrm{P}_{z}^{\nu}$ ); the other is a false-or "ghost"-solution introduced by the quadratic constraint equation. The procedure used in this analysis is to select one of the two solutions as that most likely to be the true solution. This "selected" event solution is then used for making cuts and histograms for the analysis.

The solution selection is based on the variable $\cos \alpha_{C S}$. In the Collins-Soper rest frame of the $W$ the two solutions for $\cos \alpha_{C S}$ are $e^{2} \pm\left|\cos \alpha_{C S}\right|$. The distribution of true $\cos \alpha_{C S}$ is peaked near positive 1 (figure 3.7), therefore the solution with positive $\cos \alpha_{C S}$ will be correct more often. The solution with positive $\cos \alpha_{C S}$

\footnotetext{
${ }^{2}$ The two solutions for $\phi_{C S}$ are degenerate in the CS frame. The Collins-Soper frame is unique in these properties [9].
} 
is defined to be the "selected" solution and the negative $\cos \alpha_{C S}$ solution the "rejected". The VECBOS Monte Carlo predicts that this choice is correct $73 \%$ of the time.

There is an unavoidable bias introduced by this selection method. The Monte Carlo shows that when selecting the larger value of $\cos \alpha_{C S}$ as the selected solution the smaller value of $\cos \theta^{*}$ is also chosen. There is a small region of phase space in which this isn't true; however, the Monte Carlo predicts that only one in $10^{10}$ events will fall in this category. Figure 3.9 shows the true, false, selected, and reject solutions for $\cos \theta^{*}$. The selected- $\cos \theta^{*}\left(\cos \theta_{s}^{*}\right)$ is smeared slightly to smaller values due to the selection bias.

Although the distribution of $\cos \theta_{s}^{*}$ does not match that of $\cos \theta^{*}$ it is a well defined variable. It is also sensitive to the shape of the true distribution of $\cos \theta^{*}$. As a test of this, the true $\cos \theta^{*}$ distribution generated by the VECBOS Monte Carlo was altered by re-weighting the events based on $\cos \theta^{*}$. Figure 3.10 shows the selected and true distributions for several input true $\cos \theta^{*}$ distributions. The upper plot in the figure shows the result of a flat (isotropic) $\cos \theta^{*}$ distribution. The distribution of selected $\cos \theta^{*}$ is smeared towards values of -1 ; however, it is much flatter than the distribution shown in figure 3.9. The lower plot in figure 3.10 shows the result of using a true $\cos \theta^{*}$ distribution that matches that of a spin-1 propagator (i.e. a Rutherford-type scattering distribution). These plots demonstrate that the selected $\cos \theta^{*}$ distribution tracks variations in the true $\cos \theta^{*}$ very well.

It is also interesting to look at the distribution of the variable $\left|\cos \theta^{*}\right|$. Figure 3.11 shows the true and selected $\left|\cos \theta^{*}\right|$ distributions for the same three input distributions. The selected $\left|\cos \theta^{*}\right|$ distribution matches that of the true solution quite well. This property of $\left|\cos \theta_{s}^{*}\right|$ allows it to be compared directly with $\cos \theta^{*}$ distributions measured for photon+jet and jet +jet events (which is done later in this thesis).

\subsection{Acceptance Issues}

Electrons are best measured if they go into the central part of the CDF detector; therefore, a cut is placed on the laboratory pseudo-rapidity of the electron, $\left|\eta_{\text {Lab }}\right|<$ 0.95 (for the same reason, the trigger used to accept high $\mathrm{P}_{t}$ electrons only looks in the central calorimeter. The trigger is described in section 4.7). In order to reject non- $W$ backgrounds, the electron and neutrino are required to liave transverse momentum $\left(\mathrm{P}_{t}\right)$ greater than $20 \mathrm{GeV}$. All of these cuts can cause an acceptance effect in the $\cos \theta_{s}^{*}$ distribution. The $\eta_{\text {Lab }}$ cut on the electron is the largest of these effects.

No correction will be made to the measured $\cos \theta_{s}^{*}$ distribution to remove the 

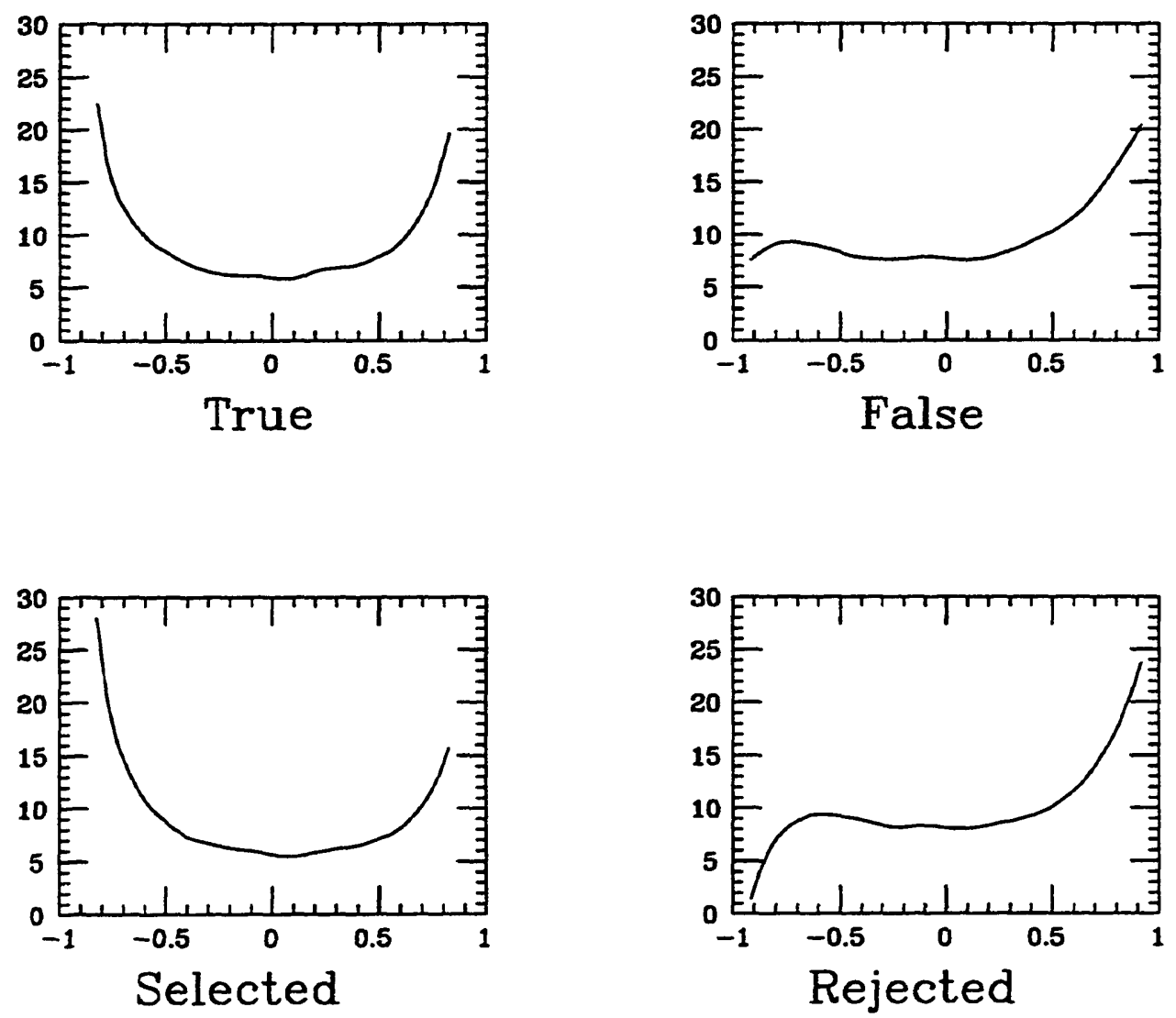

Figure 3.9: The four different distributions of $\cos \theta^{*}$ (true, false, selected, and rejected). 

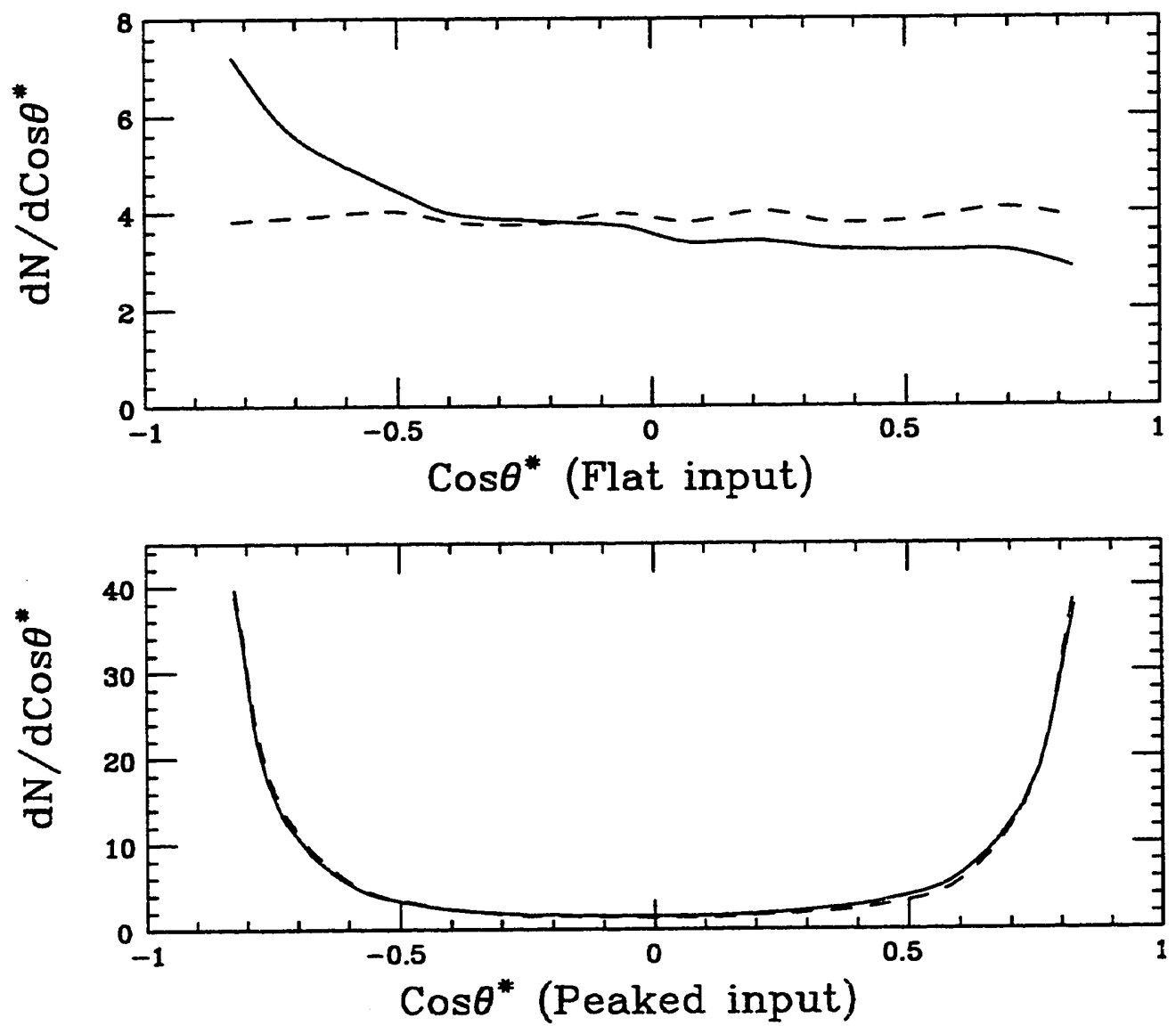

Figure 3.10: Selected and true $\cos \theta^{*}$ distributions for two altered input true $\cos \theta^{*}$ distributions. In the upper plot the input $\cos \theta^{*}$ distribution is flat. In the lower plot it is much more peaked (a spin-1 propagator). In both plots, the solid line is the selected $\cos \theta^{*}$ distribution and the dashed line is the true $\cos \theta^{*}$. 

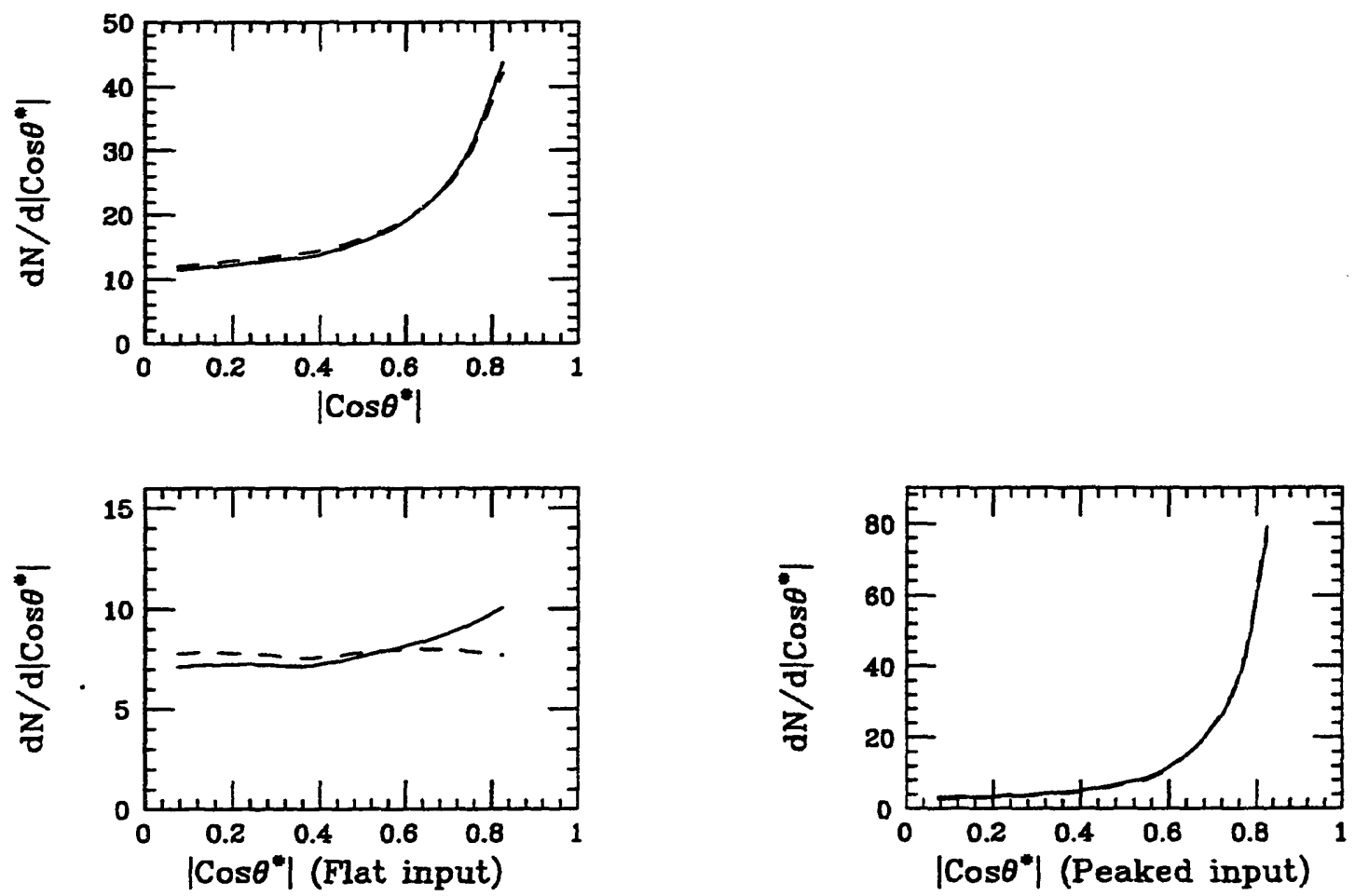

Figure 3.11: Selected and true $\left|\cos \theta^{*}\right|$ distributions. The upper plot shows $\left|\cos \theta^{*}\right|$ distributions for the VECBOS Monte Carlo. In the lower-left plot the input $\cos \theta^{*}$ distribution is flat. In the lower right plot it is much more peaked (a spin-1 propagator). In all three plots, the solid line is the selected $\left|\cos \theta^{*}\right|$ distribution and the dashed line is the true $\left|\cos \theta^{*}\right|$. 
acceptance effects from the $\eta_{\text {Lab }}$ cut. There are two reasons for not making this correction. The first is that the acceptance is predicted to be nearly flat by the VECBOS Monte Carlo, which would mean that making an acceptance correction would change the result very little. The second is that the acceptance is not flat for one of the sub-processes in VEcBOS. When the $W$ is produced by an initial state containing a down quark and a gluon the acceptance effect in $\cos \theta_{s}^{*}$ is much larger than for the other processes (the $d+g$ events only account for a small fraction of the entire sample). An acceptance correction would then be sensitive to the fraction of each sub-process that is actually being produced in the real data.

In order to compare a theoretical prediction to the final $\cos \theta_{s}^{*}$ plot, then, the prediction must include the effects of the $\eta_{\text {Lab }}$ cut on the electron. This is commented on in more detail in the concluding section of this chapter.

It is interesting to study how the $\eta_{\text {Lab }}$ cut on the electron causes an acceptance effect in $\cos \theta^{*}$. Understanding this leads to more of an understanding of why it is undesirable to attempt to correct for the acceptance. The rest of this section discusses these issues.

The $\eta_{\text {Lab }}$ of the electron is a function of several of the center-of-mass variables. The electron will tend to have a large positive $\eta_{\text {Lab }}$ (and hence be outside the calorimeter coverage) when $\cos \alpha_{C S}$ is large and positive, $Y_{\text {Boost }}$ is large and negative, and $\cos \theta^{*}$ is large and positive. Figure 3.12 shows the acceptance region in the $\cos \alpha_{C S}-Y_{\text {Boost }}$ plane for $\cos \theta^{*}=-0.9$ and for $\cos \theta^{*}=+0.9$.

If acceptance corrections were to be made, one way of doing it would be to weight each event according to an acceptance function $f\left(\cos \theta^{*}, \mathrm{M}^{*}, Y_{\mathrm{Boos}}\right)$. To construct this function, would require assuming specific distributions for $\cos \alpha_{C S}$ and $\phi_{C S}$; that is, assuming that the polarization state of the $\mathrm{W}$ is known. It can also be seen from figure 3.12 that corrections for large negative values of $Y_{\text {Boost }}$ could become very large, because only a small fraction of $\cos \alpha_{C S}$ is accepted in that range. In fact, the sub-process containing a down quark and a gluon in the initial state populates the region with $\cos \alpha_{C S}$ near 1 and $Y_{\text {Boost }}$ large and negative (this is why it's acceptance is worse than the other subprocesses, as was mentioned above).

\subsection{Transformation to True $\operatorname{Cos} \theta^{*}$}

Another correction that could be made to the final $\cos \theta_{s}^{*}$ distribution would be to unfold it, in some way, to obtain the true $\cos \theta^{*}$ distribution. Again, this correction is not applied in this analysis. This section discusses the reasons for not attempting to make this unfolding.

One method for doing this unfolding would be to develop a matrix representing 


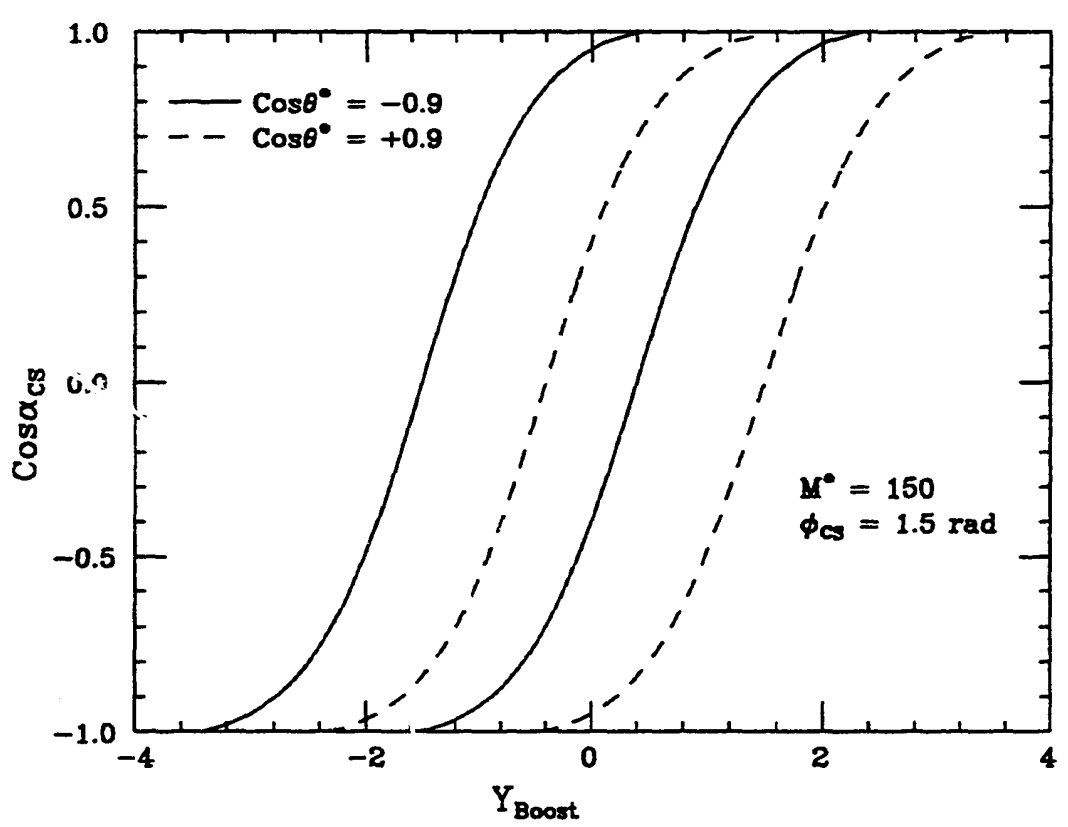

Figure 3.12: Acceptance region in the $\cos \alpha_{C S}-Y_{\text {Boost }}$ plane. The two lines represent a lab eta of -0.95 and +0.95 ; therefore the region between the lines represents events in which the electron hits the central calorimeter. 
the transformation of bins in $\cos \theta^{*}$ into bins in $\cos \theta_{s}^{*}$. This could be done using a Monte Carlo event sample that has been divided-up into bins in true $\cos \theta^{*}$. This matrix could be inverted and then multiplied onto the bins in $\cos \theta_{s}^{*}$ to get the true $\cos \theta^{*}$ distribution that produced them. One disadvantage to this method is that the unfolding matrix would have many assumptions built into it about the kinematics of the $W+$ Jet events. Comparison with a new theoretical prediction would then require first producing the selected $\cos \theta^{*}$ distribution and then operating on it with the unfolding matrix in order to compare with the data (in this case the comparison might as well be done with the selected $\cos \theta^{*}$ distribution).

It is very informative, however, to make a direct comparison between the CDF $\cos \theta^{*}$ measurement for photon + jet events and the $W+$ jet events (as well as with the jet+jet events). The comparison would be easy if the true $\cos \theta^{*}$ distribution could be obtained for the $\mathrm{W}+$ jet events. Fortunately, as was mentioned above, the distribution of $\left|\cos \theta_{s}^{*}\right|$ for the $W+$ jet events is very similar to the true $\left|\cos \theta^{*}\right|$ distribution (for the Monte Carlo events). The $\left|\cos \theta_{s}^{*}\right|$ distribution from this analysis is compared directly to the photon + jet and jet+jet results in Chapter 10 .

\subsection{Normalization of the $\operatorname{Cos} \theta^{*}$ Distribution}

The final $\cos \theta_{s}^{*}$ distribution will be presented with an arbitrary normalization factor. This emphasizes that the measurement is of the shape of the $\cos \theta_{s}^{*}$ distribution and not of the absolute cross section. The normalization scheme is chosen to be similar to CDF's previous $\cos \theta^{*}$ measurements for dijets $[10,11]$ and photon+jets[12, 13].

The normalization region is chosen to be the range from -0.6 to 0.6 . The $\cos \theta_{s}^{*}$ distribution is then normalized so that the average value of all the bins in the normalization region is 1 . One of the reasons for looking at the $W+-$ jet $\cos \theta^{*}$ distribution is to compare it with the more peaked distribution of the dijet events; normalizing in a region around zero is the best way to show how peaked the outer bins are as compared to the inner ones.

\subsection{Summary}

This chapter has presented a rough outline of the analysis method used in this thesis. It describes the properties of $W+$ Jet events, and attempts to justify the measurement of the selected $\cos \theta^{*}$ distribution.

The acceptance effects on the $\cos \theta_{s}^{*}$ distribution are small, and the folded $\left(\left|\cos \theta_{s}^{*}\right|\right)$ distribution approximates the true $\cos \theta^{*}$ distribution very well. Both of 
these facts make it fair to compare the result of this analysis directly with those of the photon + jet and jet + jet $\cos \theta^{*}$ measurements.

In order to compare a theoretical prediction precisely to the results of this thesis, it is necessary to calculate the selected $\cos \theta^{*}$ distribution including a small number of kinematic cuts (e.g. the $\eta_{\text {Lab }}$ cut on the electron). In order to do this it is essentially necessary to do the calculation using Monte Carlo techniques. Monte Carlo calculations also make it easy to calculate the two neutrino solutions and to pick the selected solution (something quite difficult to do analytically). The decision to present a result that necessitates a Monte Carlo calculation for comparison was based on the fact that nearly all theoretical predictions being made at this time are, in fact, in the form of Monte Carlo calculations (that is, Monte Carlo calculations are the only practical way of doing the predictions anyway). A good example of this is the recent next-to-leading order $W+$ jet calculation released in the Monte Carlo program DYRAD[14] (the predictions from this Monte Carlo program will be compared to the data in Chapter 10). It should be emphasized that the Monte Carlo calculations, needed to compare with the results presented in this thesis, do not require any special knowledge of the CDF detector, and do not involve any energy smearing (i.e. detector resolution). 


\section{Chapter 4}

\section{CDF Detector}

CDF (Collider Detector at Fermilab) is a large multi-purpose detector that surrounds the $\mathrm{B} 0$ interaction region of the Fermilab Tevatron. It has good electron identification abilities out to a pseudo-rapidity $(\eta)$ of 1.0 , good muon identification out to $\eta$ of 0.6 , and nearly hermetic calorimetry to $\eta$ of 4.2 . This chapter will describe those components of the detector used in this analysis. The detector is described in greater detail in [15] and its references.

CDF was designed for testing many different predictions of the Standard Mode! and also to search for processes beyond the Standard Model. In this spirit the detector was designed to measure the properties of a wide range of possible finalstate particles. Figure 4.1 shows a cut-away view of the detector. CDF provides tracking and momentum measurement for charged particles. It has calorimetry for the energy measurement of leptons, hadrons, photons, and jets. Combinations of the different detector components can be used quite efficiently for particle identification; CDF routinely identifies electrons, muons, photons, and (much less efficiently) taus. Due to the hermetic calorimeter coverage it is even possible to detect the presence of large transverse-momenta neutrinos (from the transverse momentum imbalance that results in the calorimeters).

In order to provide such general-purpose measurement, CDF is a combination of several detector components. Starting at the collision point (of the proton and anti-proton) and moving outward, the final-state particles first traverse a thin beryllium window in the Tevatron beam-pipe. They then traverse tracking chambers that measure the position and momentum of the charged particles. Outside the tracking chambers is a superconducting magnet which immerses the tracking chambers in a 1.5 Tesla solenoidal magnetic field. Surrounding the magnet are calorimeters for measuring the energy of the particles. Beyond the calorimeters are muon detectors.

The following sections describe the Fermilab collider and each of the CDF detector components in rnore detail. 


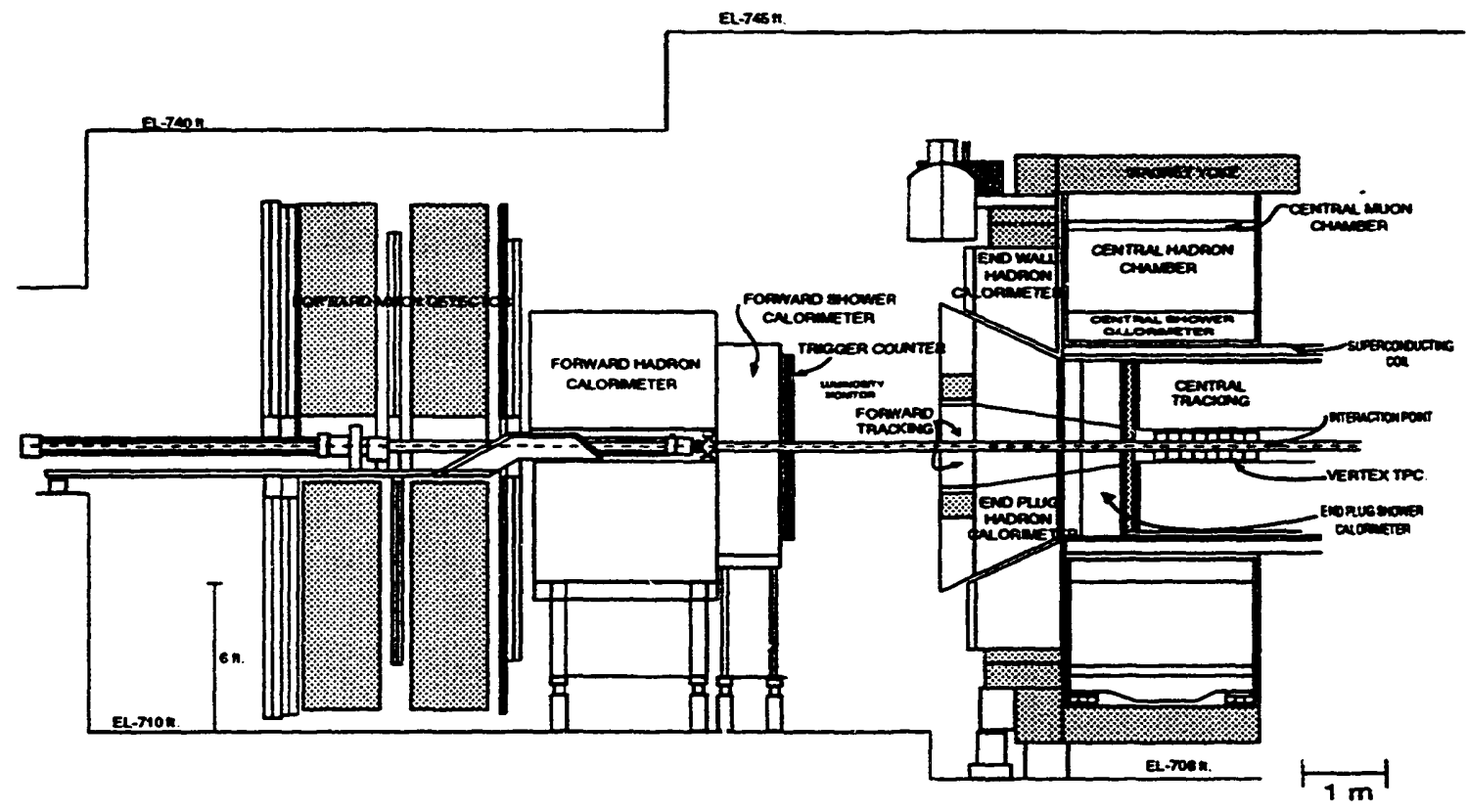

Figure 4.1: A cut-away view of the forward half of the CDF detector. The detector is forward/backward symmetric. 


\subsection{Fermilab Collider}

A schematic diagram of the Tevatron is shown in figure 4.2. This section is a very schematic description of the operation of the collider.

The acceleration process begins by accelerating ionized hydrogen atoms (one electron is added) in a linear accelerator to $200 \mathrm{MeV}$ (in the process the electrons are stripped from the proton). The Booster Ring then accelerates the protons to 8 $\mathrm{GeV}$ and injects them into the Main Ring. The main ring accelerates the protons to $150 \mathrm{GeV}$. The protons can then be transferred to the super-conducting Tevatron (which lies beneath the Main Ring). The Tevatron accelerates the protons to their final energy of $900 \mathrm{GeV}$.

Protons from the Main Ring can be sent to collide with a metal target. In these collisions many anti-protons are produced. These are collected and stored in the anti-proton storage ring. The storage ring is used to stochastically cool the anti-protons and then store them until enough have been accumulated for injection into the Tevatron.

The protons and anti-protons are injected into the Tevatron in 6 bunches. Quadrapole magnets on each side of CDF focus the beams at the center of the detector. The distribution of the collision point in $z$ is gaussian with a width of $35 \mathrm{~cm}$.

\subsection{Vertex Time Projection Chamber}

Surrounding the beam-pipe are the vertex time projection chambers (VTPC). As mentioned in the last section, the proton anti-proton collisions can occur along a large section of the beam-line. The VTPC provides tracking information that gives a good $\mathrm{z}$ and theta measurement, which is then used to determine the position of the interaction point (or points in the case of multiple collisions).

Another important property of the VTPC system is that it provides efficient tracking information at a point where there is very little material between the beam and the measurement. Photons produced at the collision point can convert into electron pairs when passing through detector material. An efficient method for removing photons which convert outside the VTPC is to look for the electron tracks in the VTPC.

The VTPC consists of eight TPC modules placed end-to-end along the beam line. Figure 4.3 shows a schematic drawing of two of the VTPC modules. Each of the modules is $35 \mathrm{~cm}$ long. They are divided in half by a high-voltage screen which creates two $15.25 \mathrm{~cm}$ long drift regions. The modules are filled with an argon-ethane mixture; when a charged particle passes through a module it leaves 


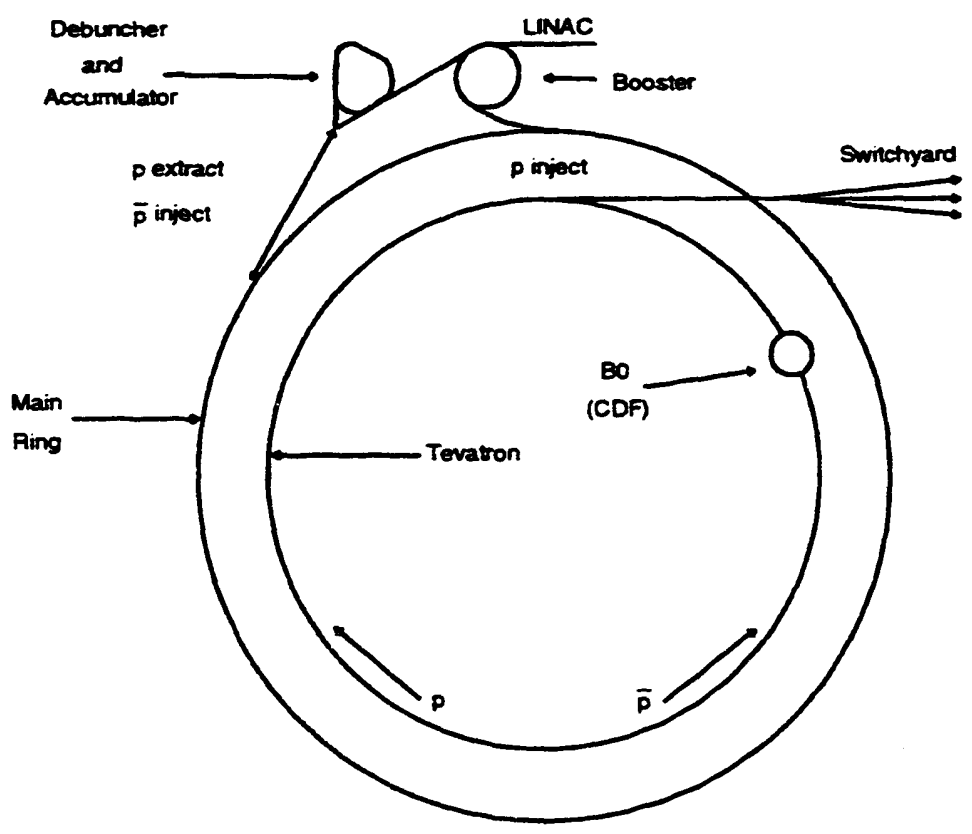

Figure 4.2: A schematic diagram of the Fermilab accelerator complex. 
an ionization trail that then drifts away from the central HV screen to the ends of the module.

Each chamber's endcap is divided into multi-wire proportional chamber octants; see figure 4.3. The arrival time of the track ionization at each of the octant's sense wires determines the track in $r-z$ space ( $r$ being the radius from the beam).

It is possible to get some $\phi$ information from the VTPC modules for low angle tracks $\left(5^{\circ}\right.$ to $\left.25^{\circ}\right)$. Some of the VTPC modules' cathode pads are equipped with ADC's and can be read out. Also, adjacent VTPC modules are rotated by $11.31^{\circ}$ in $\phi$ so that tracks passing through two modules will have some $\phi$ information.

Finally, it should be noted that the construction of the VTPC was done with low mass materials wherever possible. This reduces the photon conversions and multiple scattering caused by the VTPC. The VTPC is constructed mainly of Rohacell foam, Kapton film, and epoxy-glass or epoxy-graphite laminates.

Table 4.1 shows a summary of the VTPC parameters. Figure 4.4 shows a graphic display of the VTPC output for a typical W + jet event.

\subsection{Central Tracking Chamber}

It is important in hadron collisions to be able to reconstruct charged particle tracks. At a very basic level tracks show the history of a particle. We are usually only interested in particles that come directly from the primary event vertex. Counter examples are cosmic ray particles or particles that result from the decay of another long lived particle.

In combination with a magnetic field, a particle track determines the charge and momentum of the particle (assuming, of course, that it has a charge $\pm e$ ). Knowing the charge of particles is extremely important for many physics analyses. For example a top quark decay can produce two leptons with opposite charge, and is much less likely to produce two leptons with the same charge. The presence of a track with momentum consistent with calorimeter energy deposition can distinguish an electron from a photon (electron identification will be discussed in detail later).

The central tracking chamber (CTC) is a cylindrical drift chamber 2.6 meters in diameter and 3.2 meters in length. It provides three-dimensional tracking and high-resolution momentum measurement for the angular region $-1<\eta<1$. The resolution for the CTC is $\delta p_{T} / p_{T}^{2}=0.0011$ for tracks constrained to come from the known $\mathrm{x}$-y position of the Tevatron beam $\left(\delta p_{T} / p_{T}^{2}=0.002\right.$ without the beam constraint).

The chamber consists of 84 layers of sense wires. The layers are grouped into 9 "superlayers". Layers $1,3,5,7$, and 9 have 12 axial sense wires. Layers $2,4,6$, and 


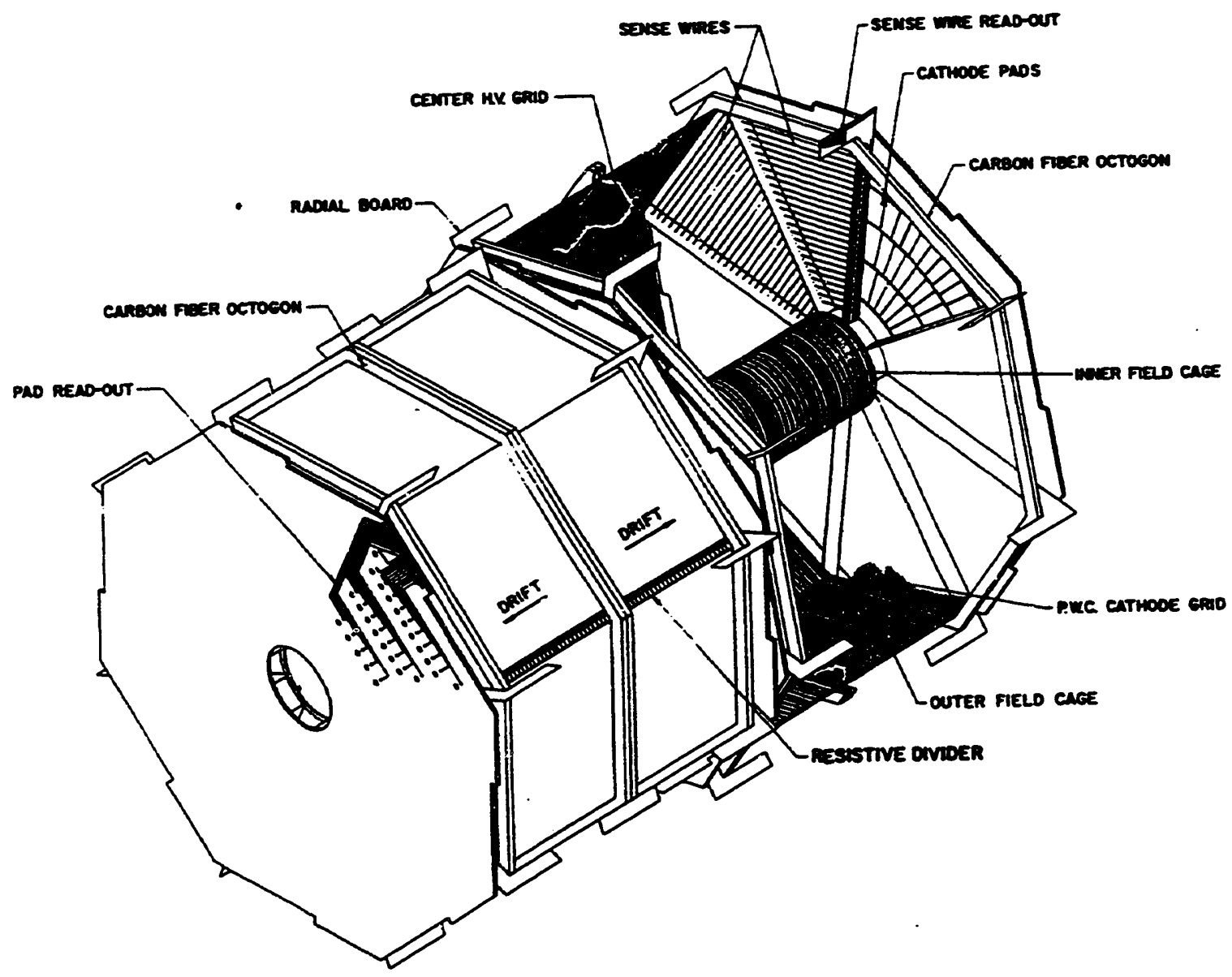

Figure 4.3: An isometric view of two VTPC modules. 


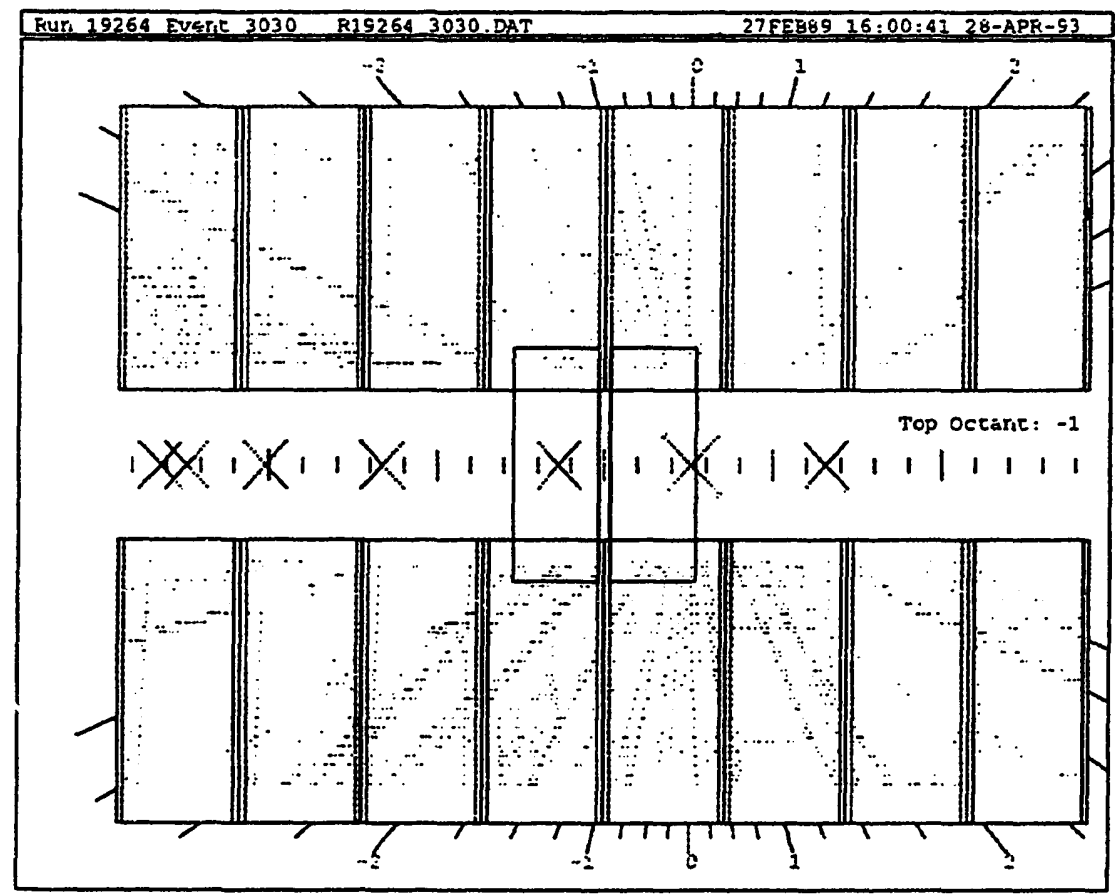

Figure 4.4: An event display showing an $\mathrm{r}-\mathrm{z}$ projection of the VTPC wire hits for a typical $W+$ jet event. The large crosses along the beamline mark vertices (most of which are based on only one track). The vertex for the $W+$ Jet event is shown by the cross with bars on the end and is at $z=25.9 \mathrm{~cm}$. The tic marks along the outside of the modules show pseudo-rapidity. 


\begin{tabular}{||lc||}
\hline Angular coverage: & $3.5^{\circ}<\theta<176.5^{\circ}$ \\
Inner & $-3.5<\eta<3.5$ \\
& $8.7^{\circ}<\theta<171.3^{\circ}$ \\
Outer & $-2.6<\eta<2.6$ \\
\hline Mechanical parameters: & \\
Number of modules & 8 \\
Octants per module & 16 \\
Wires per octant & 24 \\
Pads per octant & 24 \\
Module length & $35.3 \mathrm{~cm}$ \\
Module spacing & $35.94 \mathrm{~cm}$ \\
Outer active radius & $21 \mathrm{~cm}$ \\
Inner active radius & $7 \mathrm{~cm}$ \\
Drift length & $15.25 \mathrm{~cm}$ \\
Drift field & $256 \mathrm{~V} / \mathrm{cm}$ \\
Magnetic field & $1.5 \mathrm{~T}$ \\
Gas & $50 / 50 \mathrm{Argon}-\mathrm{ethane}$ \\
Drift velocity & $42 \mu \mathrm{m} / \mathrm{ns}$ \\
Sense wires & $6.336 \mathrm{~mm}$ \\
Sense wire spacing & $200-500 \mu \mathrm{m}$ \\
\hline Resolution: & $6 \mathrm{~mm}(\mathrm{r}-\mathrm{Z}), 3 \mathrm{~cm}(\phi)$ \\
Spatial &
\end{tabular}

Table 4.1: Vertex Time Projection Chamber (VTPC) Specifications 
8 have 6 sense wires tilted by an angle of $\pm 3^{\circ}$ from the $z$-axis; these layers are called the "stereo superlayers" because they provide a $\mathrm{z}$ position measurement at 4 points along the track.

Figure 4.5 shows a diagram of the CTC endplate showing the angle of each sense wire cell (what's shown is actually the slots cut in the aluminum end-plate). The sense wire cells are tilted at an angle of $45^{\circ}$ to allow for the drift direction induced by the 1.5T B field.

Figure 4.6 shows a graphics display of the CTC information for a typical CDF $W+$ jet event.

\subsection{Magnet}

The VTPC and the CTC are immersed in a 1.5 Tesla solenoidal magnetic field (directed along the $\mathrm{z}$ axis). This field is provided by a superconducting solenoidal coil on the outside of the CTC. The magnet is 3 meters in diameter and 5 meters long, and consists of 1164 turns of aluminum-stabilized $\mathrm{NbTi} / \mathrm{Cu}$.

The solenoid is supported on the outside by a thin aluminum-alloy cylinder (this support structure is chiefly to counteract the outward magnetic force). The support cylinder has an aluminum tube welded to it through which two-phase helium is forced to cool the magnet. The combined magnet and support structure is 0.85 radiation lengths thick.

\subsection{Calorimetry}

Outside the tracking chambers, out to an $\eta$ of 4.2 (hermetic to $\eta=3.6$ ) are the calorimeters. Closest to the interaction point are electromagnetic calorimeters that measure the energy of electrons and photons. Behind the EM calorimeters are the hadron calorimeters. All of the calorimeters are segmented into projective towers which point back to the nominal interaction point. The towers are approximately 0.1 units wide in pseudo-rapidity. In the central region $(|\eta|<1.1)$ the towers are $15^{\circ}$ wide in $\phi$, and in the plug $(1.1<|\eta|<2.4)$ and forward $(2.4<|\eta|<4.2)$ regions they are $5^{\circ}$ wide in $\phi$. The following sections describe the calorimetry in each of these $\eta$ regions.

\subsubsection{Central Calorimeters}

The central calorimeters are constructed from "wedges", each of which covers the $\eta$ range from 0 to 1.1 (or -1.1 to 0 ) and $15^{\circ}$ in $\phi$. The wedges are assembled into 


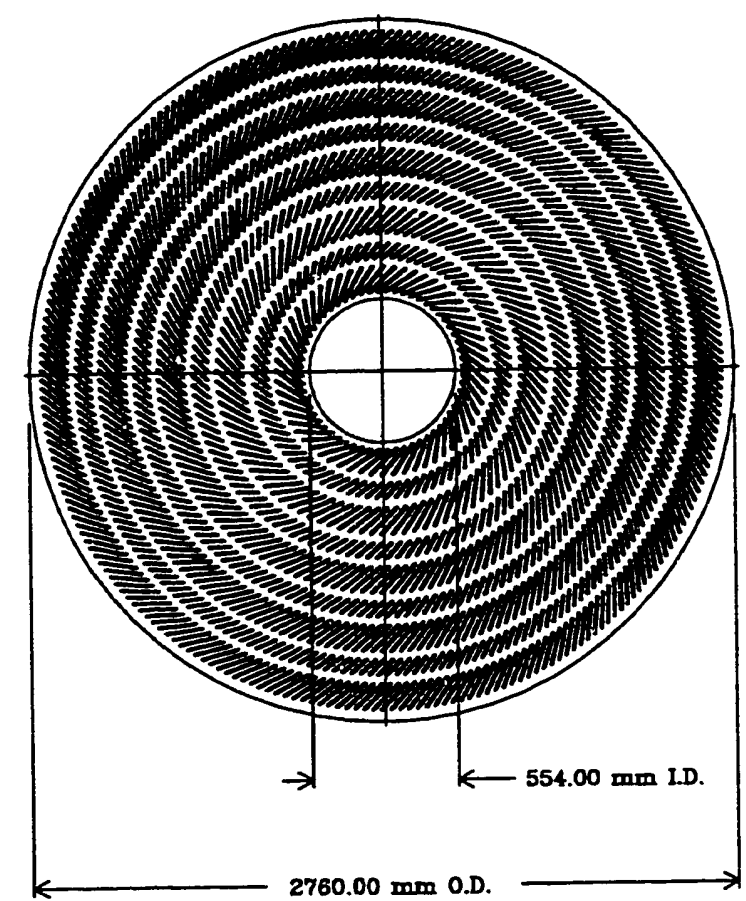

Figure 4.5: End view of the CTC endplate showing the wire slots.

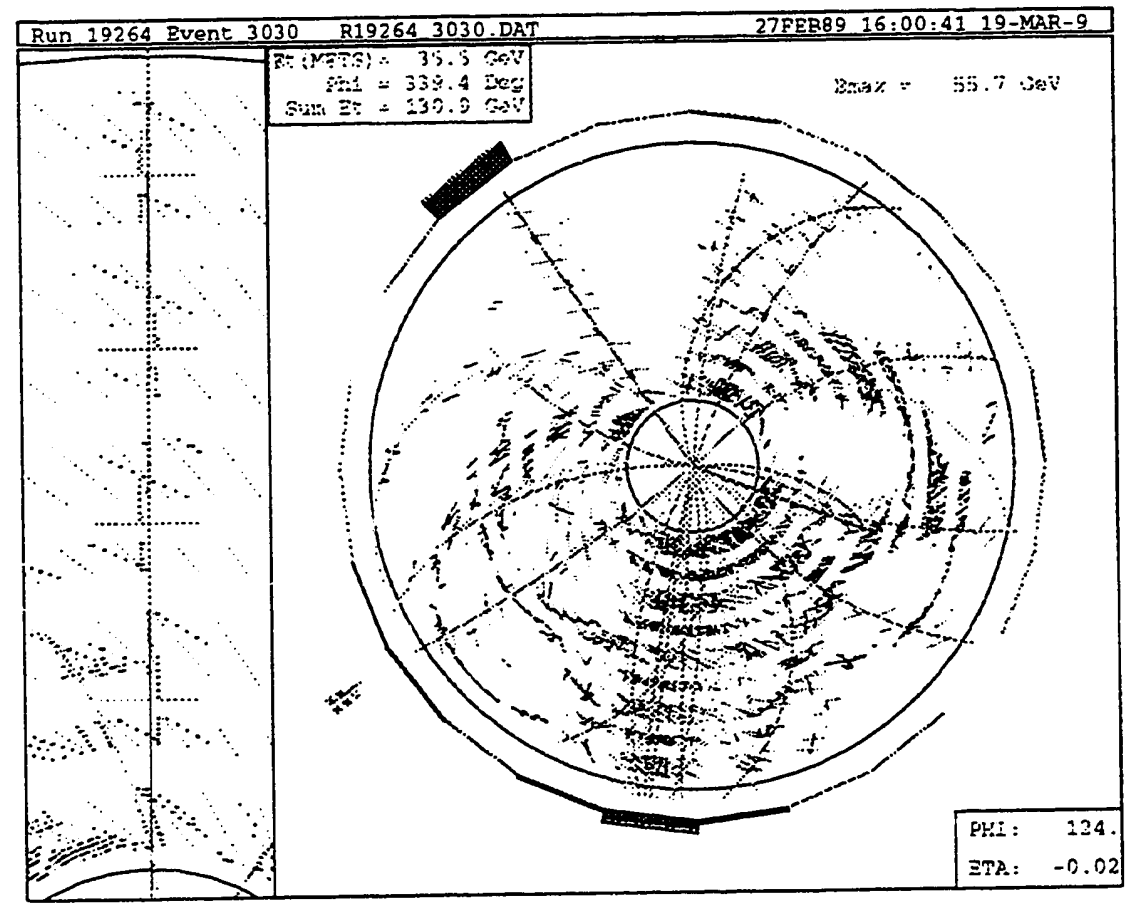

Figure 4.6: An event display showing a typical $W+$ jet event's hits in the CTC. The reconstructed tracks are also shown. The electron is in the upper left. The box on the left shows an enlarged view of the electron's track (the $x$ and $y$ scales are not the same in this magnified view). 


\begin{tabular}{|lc||}
\hline Angular coverage: & $15^{\circ}<\theta<165^{\circ}$ \\
Inner & $-2.0<\eta<2.0$ \\
& $40^{\circ}<\theta<140^{\circ}$ \\
Outer & $-1.0<\eta<1.0$ \\
\hline Mechanical Parameters: & 84 \\
Number of layers & 9 \\
Number of superlayers & 12 \\
Layers/axial superlayer & 6 \\
Layers/stereo superlayer & $0^{\circ},+3^{\circ}, 0^{\circ},-3^{\circ}, 0^{\circ},+3^{\circ}, 0^{\circ},-3^{\circ}, 0^{\circ}$ \\
Stereo angle & $30,42,48,60,72,84,96,108,120$ \\
Number of supercells/layer & $309 \mathrm{~mm}$ \\
Radius inner & $1320 \mathrm{~mm}$ \\
Radius outer & $3214.0 \mathrm{~mm}$ \\
Wire length & $1.5 \mathrm{Tesla}$ \\
Mag field & $1350 \mathrm{~V} / \mathrm{cm}$ \\
Drift field & $50 / 50 \mathrm{Argon}-\mathrm{ethane}$ \\
Gas & $40 \mathrm{~mm}$ \\
Maximum drift distance & $>0.98 \mathrm{per} \mathrm{point}$ \\
Efficiency & $<P_{t} / P_{t}^{2}=0.0011$ \\
\hline Resolution: & $3.5 \mathrm{~mm}$ \\
Spatial r- $\phi$ & $200 \mu \mathrm{m} /$ wire \\
Spatial Z & $4 \mathrm{~mm}$ \\
Momentum & \\
Two track & \\
\hline
\end{tabular}

Table 4.2: Central Tracking Chamber (CTC) Specifications 
4 self-supporting "arches" (each 1.1 units in $\eta$ and $180^{\circ}$ in $\phi$ ) which can be pulled apart for servicing or access to the inner detector components.

\section{Central Electromagnetic Calorimster}

The central electromagnetic calorimeter (CEM) consists of alternating layers of lead and a polystyrene scintillator. Electrons and photons that interact with the lead will cause an electromagnetic shower (an exponential growth of conversion pairs and bremsstrahlunged photons). The total number of particles created in this shower will be proportional to the energy of the incoming particle; therefore the number of photons coming out of the scintillator will be proportional to the initial particle's energy.

The light from the scintillator is captured in wave-shifter plates mounted on the sides of the calorimeter. Figure 4.7 shows a schematic drawing of the CEM which emphasizes the light-gathering system. The wave-shifter plates redirect the light into acrylic light-guides which carry the light past the hadronic part of the calorimeter to photomultiplier tubes at the end of the calorimeter. The calorimeter is sectioned into ten projective towers, each 0.1 units in $\eta$. Each of these towers has two PMT's (one on each side).

To further aid in identifying electrons and photons a high-resolution gas multiwire proportional chamber (CES \{Central Electron Strips\}) is positioned 6 radiation lengths into the CEM. The CES provides a measurement of the transverse shape of the electromagnetic shower (which is very narrow for electrons) and also provides an accurate measurement of its position. The CES cathode consists of strips that run orthogonal to the wires; therefore both $\phi$ and $\mathrm{Z}$ information are available for the shower. Figure 4.8 shows a schematic diagram of a CES module.

\section{Central Hadronic Calorimeter}

The central hadronic calorimeter ( $\mathrm{CHA}$ ) consists of alternating layers of steel and acrylic scintillator. The calorimeter is sectioned into ten $\eta$ towers that match the CEM towers. The light from the scintillator is carried to PM tubes by wave-shifter strips along the length of each scintillator layer. Figure 4.9 shows how the light is collected from each layer. Figure 4.10 shows a schematic drawing of one CHA wedge.

\section{Wall Hadronic Calorimeter}

The wall hadron calorimeter (WHA) is constructed using the same methods and materials as the CHA. The WHA is needed, because of the constraints imposed by 


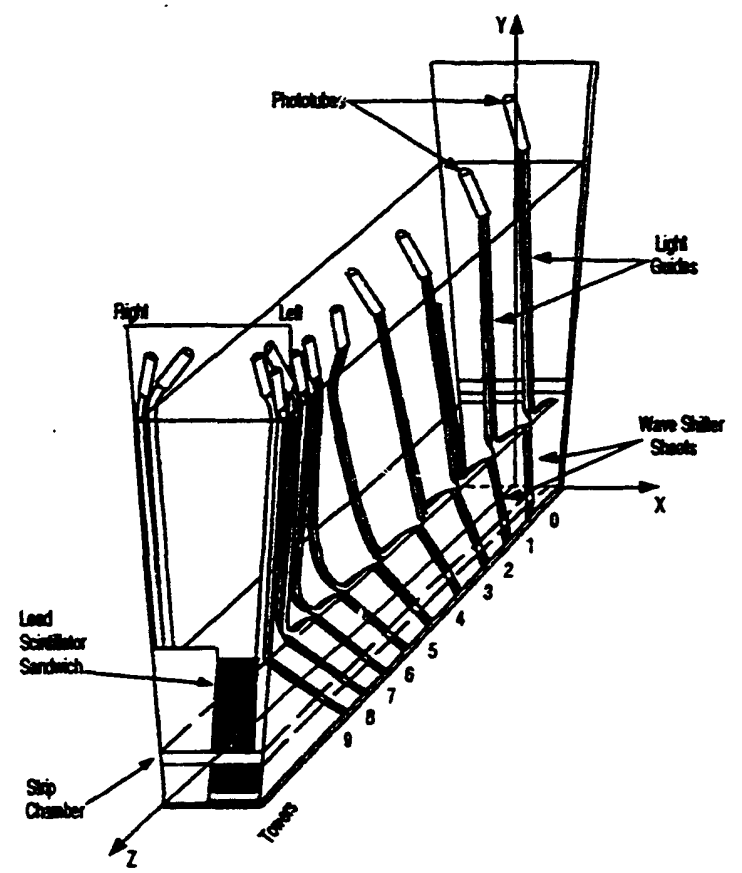

Figure 4.7: Layout of the light-gathering system for the CEM.

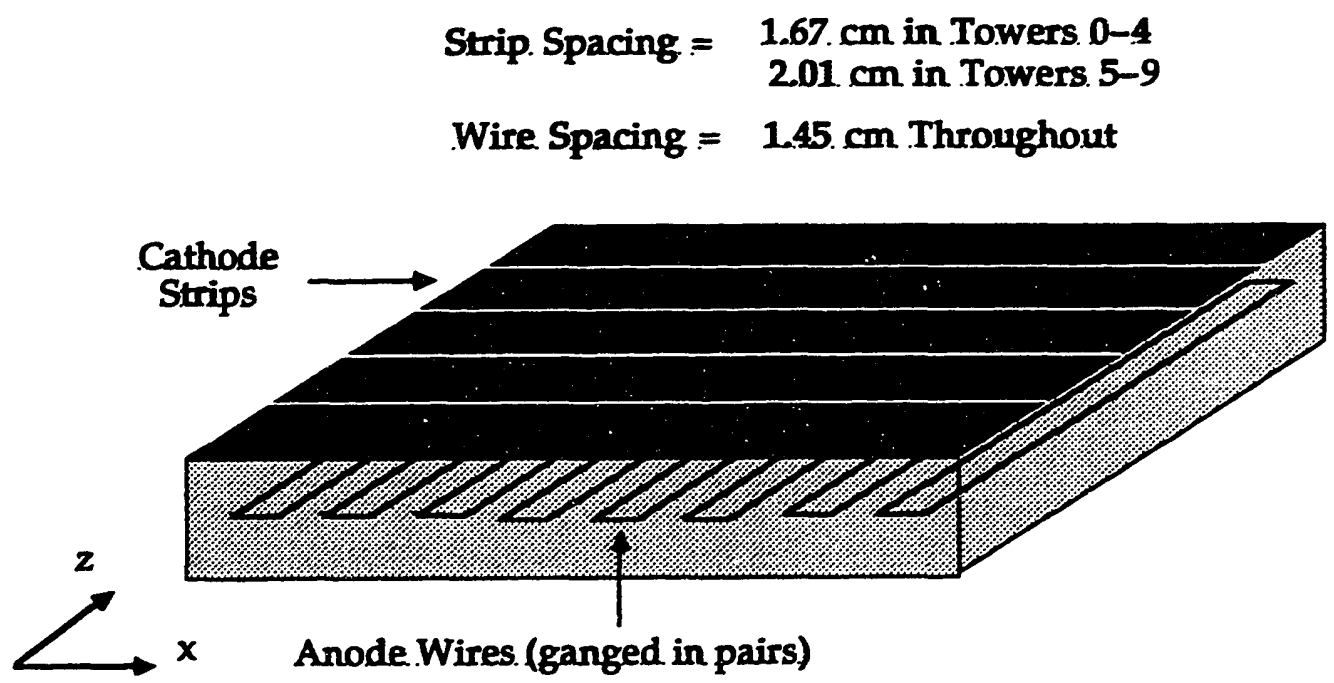

Figure 4.8: A schematic diagram of a CES chamber. 


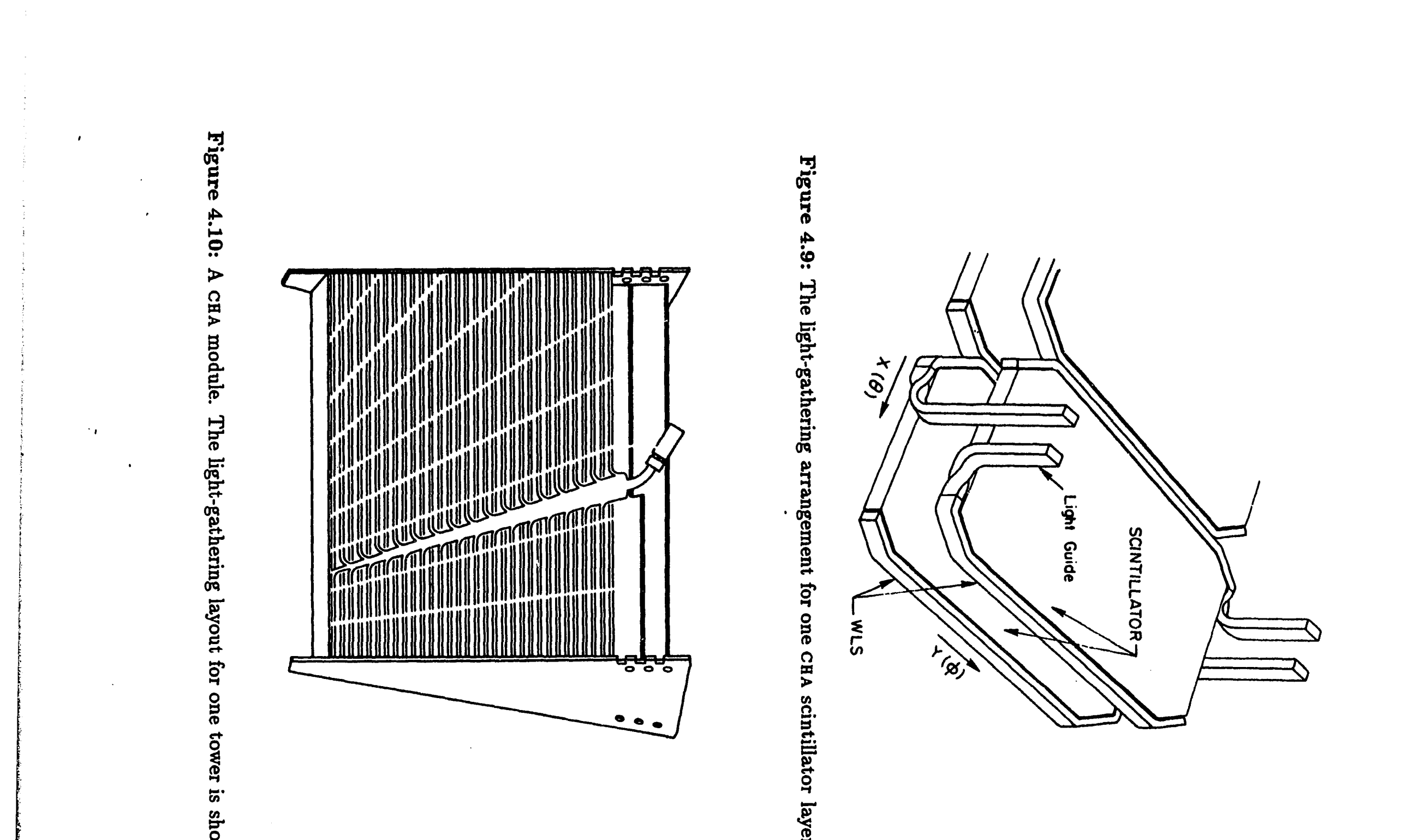


the solenoid, to complete the hadronic coverage for both the CHA and the plug hadronic calorimeter (to be discussed in the next section), see figure 4.1.

One of the central wedges has a hole in it to allow for cryogenic plumbing to the solenoid. The hole covers the $\eta$ range from approximately 0.7 to 0.8 . This wedge is termed the "chimney" module.

The central calorimeters were calibrated in a fixed-target test beam at Fermilab. The test beam provides electrons or pions tunable over a wide range of energies. The CEM has a built-in remote-controlled radiation source and two different light sources all of which provide in situ calibration monitoring.

Table 4.3 is a summary of the central calorimeter specifications.

\subsubsection{Plug Calorimeters}

The plug calorimeters are so named because they "plug" the ends of the superconducting magnet (the steel in the hadronic calorimeter serves as the return yoke for the magnetic field). They cover the $\eta$ range from 1.1 to 2.4. Both calorimeters employ gas multi-wire proportional chambers as the shower counting material. This calorimeter is much closer to the beamline, therefore a plastic scintillator could not be used as the active material-it would quickly be damaged by the high radiation levels.

Both calorimeters use multi-wire proportional chambers (MWPC) constructed from conductive rectangular plastic tubes (the plastic is impregnated with finegrained carbon). Each wire runs through the center of a tube which is sandwiched between two copper planes. One of the copper planes is grounded, the other is divided into pads ( 0.09 units in $\eta \times 5^{\circ}$ in phi) that are connected to ground through charge-integrating amplifiers. Figure 4.11 shows the proportional tube assembly for the electromagnetic calorimeter.

\section{Plug Electromagnetic Calorimeter}

The plug electromagnetic calorimeter (PEM) is cylindrical in shape (see figure 4.1) with a conical hole in the center corresponding to an $\eta$ of $2.4\left(10^{\circ}\right)$. It consists of 34 layers of lead and gas MWPC's. The cathode pads all form projective towers that point back to the nominal vertex. The pad signals from the first 5 layers are ganged together (summed) and read out, the signal from layers 6-29 are also ganged as are layers 30-34. Having these three samplings provides information about the longitudinal development of the electromagnetic shower (which is useful for rejecting non-electron/photon events).

The PEM boards are assembled in $90^{\circ}$ quadrants; see figure 4.12. The wires in each $90^{\circ}$ layer are electrically connected and the energy in the full $90^{\circ}$ is read out. 


\begin{tabular}{|c|c|c|c|}
\hline & CEM & CHA & WHA \\
\hline Coverage $(|\eta|)$ : & $0.0-1.1$ & $0.0-0.9$ & $0.7-1.3$ \\
\hline Tower Size $(\Delta \eta \times \Delta \phi)$ & $\sim 0.1 \times 15^{\circ}$ & $\sim 0.1 \times 15^{\circ}$ & $\sim 0.1 \times 15^{\circ}$ \\
\hline Active Medium & $\begin{array}{l}\text { SCSN-38 } \\
\text { polystyrene }\end{array}$ & $\begin{array}{l}\text { Doped PMMA } \\
\text { acrylic }\end{array}$ & $\begin{array}{l}\text { Doped PMMA } \\
\text { acrylic }\end{array}$ \\
\hline Thickness & $0.5 \mathrm{~cm}$ & $1.0 \mathrm{~cm}$ & $1.0 \mathrm{~cm}$ \\
\hline Layers & $21-31$ & 32 & 15 \\
\hline Absorber & $\mathrm{Pb}$ & $\mathrm{Fe}$ & $\mathrm{Fe}$ \\
\hline Thickness & $0.32 \mathrm{~cm}$ & $2.5 \mathrm{~cm}$ & $5.1 \mathrm{~cm}$ \\
\hline Layers & $20-30$ & 32 & 15 \\
\hline Number of modules & 48 & 48 & 48 \\
\hline Module length $(Z)$ & $250 \mathrm{~cm}$ & $250 \mathrm{~cm}$ & $110 \mathrm{~cm}$ \\
\hline Module width $(\phi)$ & $45.5 \mathrm{~cm}(\mathrm{r}=173 \mathrm{~cm})$ & $133 \mathrm{~cm}$ & $35-78 \mathrm{~cm}$ \\
\hline Module depth(r) & $34.5 \mathrm{~cm}$ & $131 \mathrm{~cm}$ & $110-170 \mathrm{~cm}$ \\
\hline $\begin{array}{l}\text { Energy Resolution } \\
\left(\frac{\sigma}{E(G e V)}\right)\end{array}$ & $\frac{13.5 \%}{\sqrt{E}} \oplus 2 \%$ & $\frac{30 \%}{\sqrt{E}} \oplus 3 \%$ & $\frac{80 \%}{\sqrt{E}} \oplus 3 \%$ \\
\hline
\end{tabular}

Table 4.3: Central Calorimeter Specifications

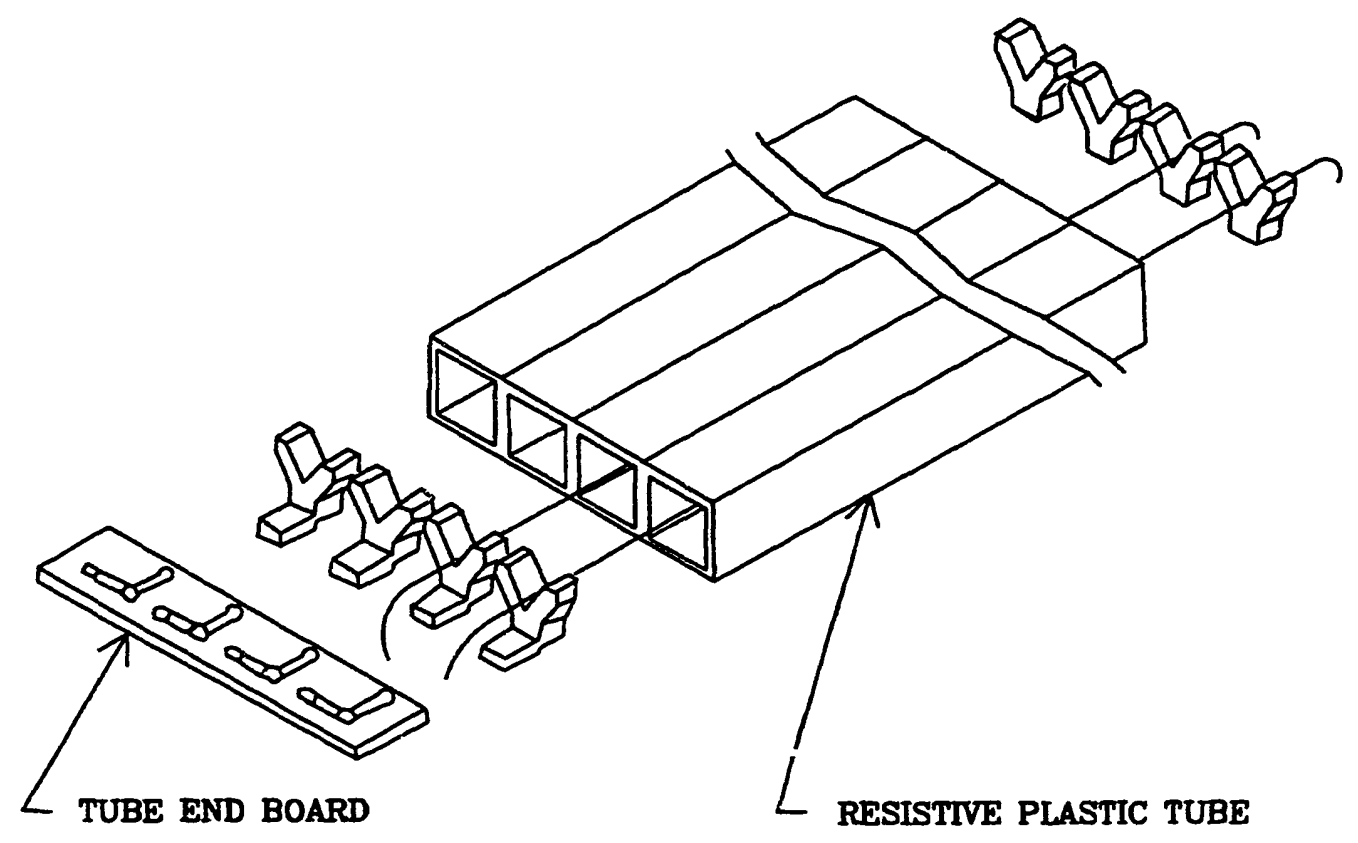

Figure 4.11: Construction of the plug proportional chambers. The PEM is shown; however, the PHA has a similar construction. 
If the electron/photon candidate accounts for most of the energy in the quadrant, then the wire signals provide a detailed longitudinal profile of the shower.

The PEM also has a high-resolution strip chamber (PES) positioned near the expected maximum of an electromagnetic shower. The PES is not used in this analysis, and the reader should refer to the reference for more detail.

\section{Plug Hadronic Calorimeter}

The plug hadronic calorimeter (PHA) consists of 20 layers of steel and gas MWPC's. All 20 cathode pads in each of the PHA towers are ganged together. The layers are constructed in $30^{\circ}$ sectors, and one wire signal is read out for each layer. Again, if the energy in one $30^{\circ}$ sector is isolated then the wire signals provide an excellent longitudinal profile of the hadronic shower. The wire signals are useful for removing large single-layer energy depositions that can occur if a proton (created by a neutron from the hadronic shower) is scattered parallel to one of the wires (referred to as "Texas towers").

Table 4.4 gives a summary of the plug calorimeter specifications.

\subsubsection{Forward Calorimeters}

The forward calorimeters are shown in figure 4.1. They are positioned approximately 6.5 meters from the nominal interaction point, and cover the $\eta$ range from 2.2 to 4.2 . Both calorimeters are gas multi-wire proportional chambers. Their construction is similar in philosophy to the plug calorimeters, the main difference being that the tubes enclosing the wires are made of aluminum. The aluminum tubes are glued to the pads with a conductive epoxy. Figure 4.13 shows the construction of the electromagnetic calorimeter's wire chambers.

\section{Forward Electromagnetic Calorimeter}

The forward electromagnetic calorimeter (FEM) is rectangular in shape and consists of 30 layers of lead and gas MWPC's. The cathode pads are ganged into two depth segments, each consisting of 15 layers. The layers are constructed in $90^{\circ}$ quadrants; figure 4.14 shows the pad layout for one of the chambers.

\section{Forward Hadronic Calorimeter}

The forward hadronic calorimeter (FHA) is also rectangular in shape and consists of 27 layers of steel and MWPC's. The pad signals for all 27 layers in each tower are ganged together. The chambers are constructed in $90^{\circ}$ quadrants; figure 4.15 shows the pad layout for one of the FHA chambers. 


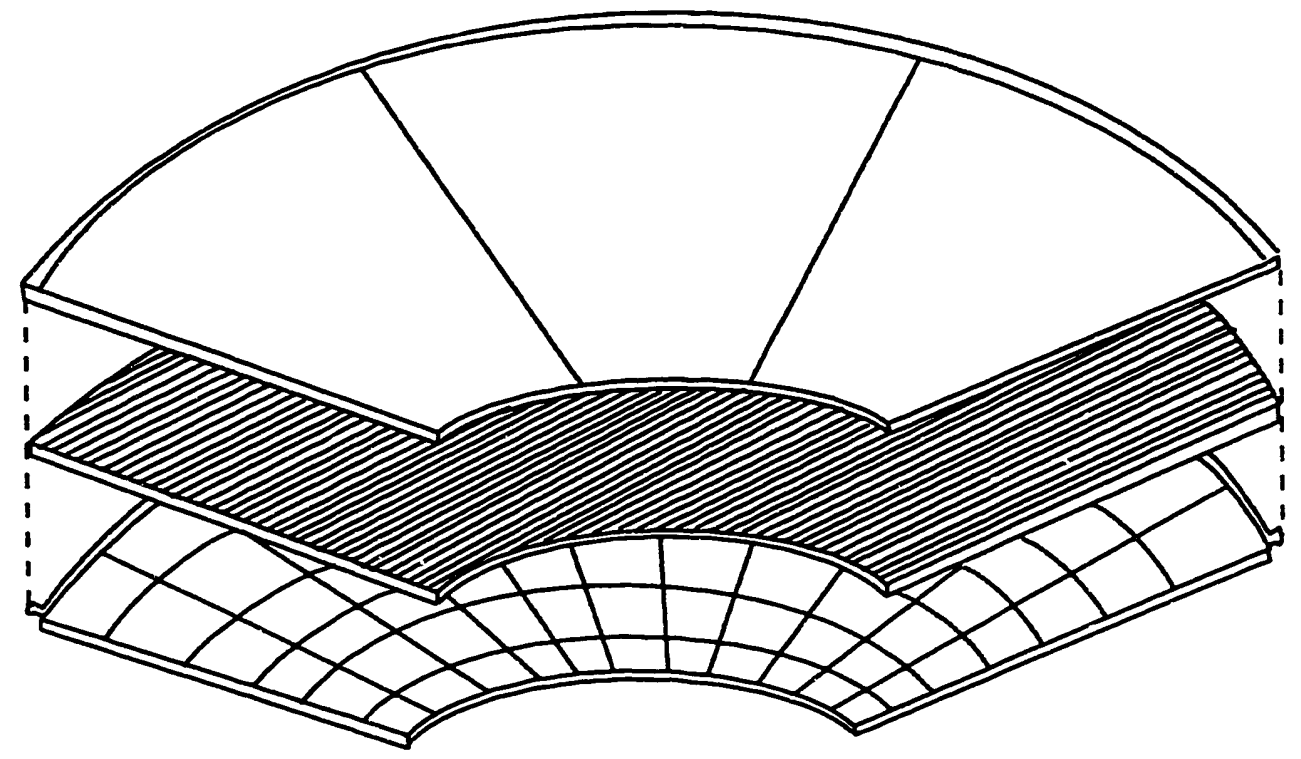

Figure 4.12: Exploded view of a PEM layer showing the proportional tubes, the pad layer, and the ground plane.

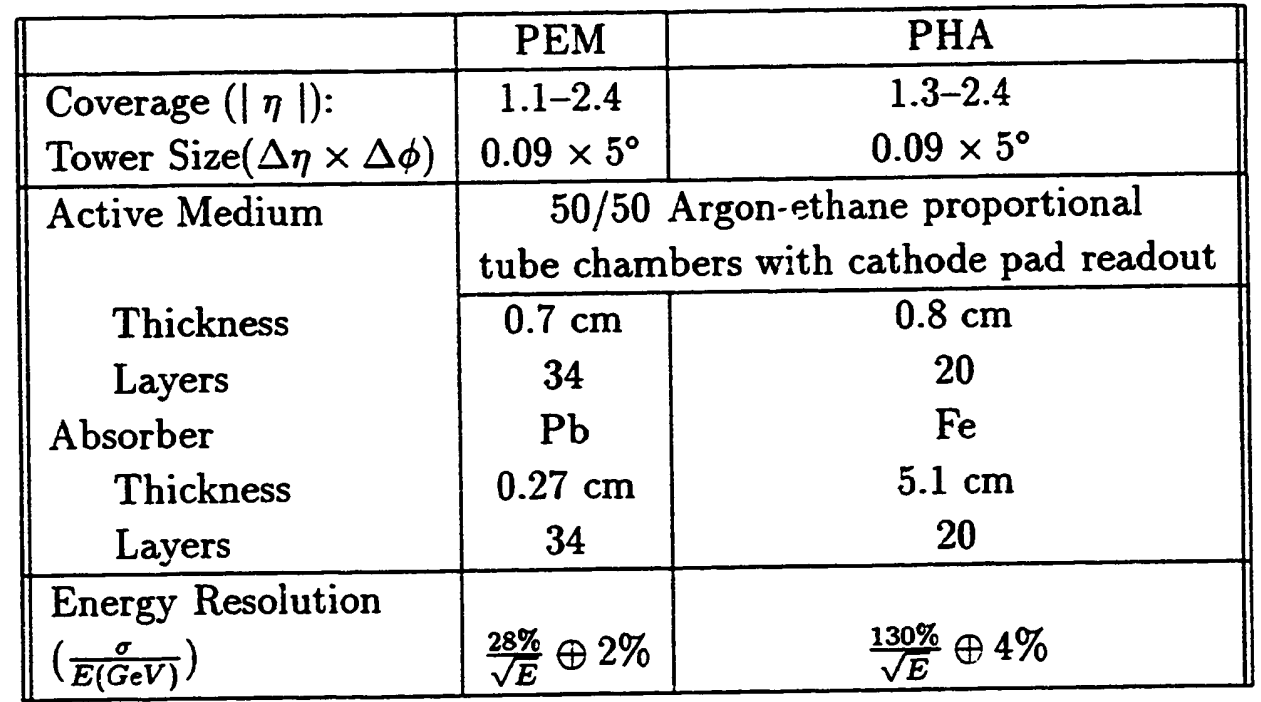

Table 4.4: Plug Calorimeter Specifications 


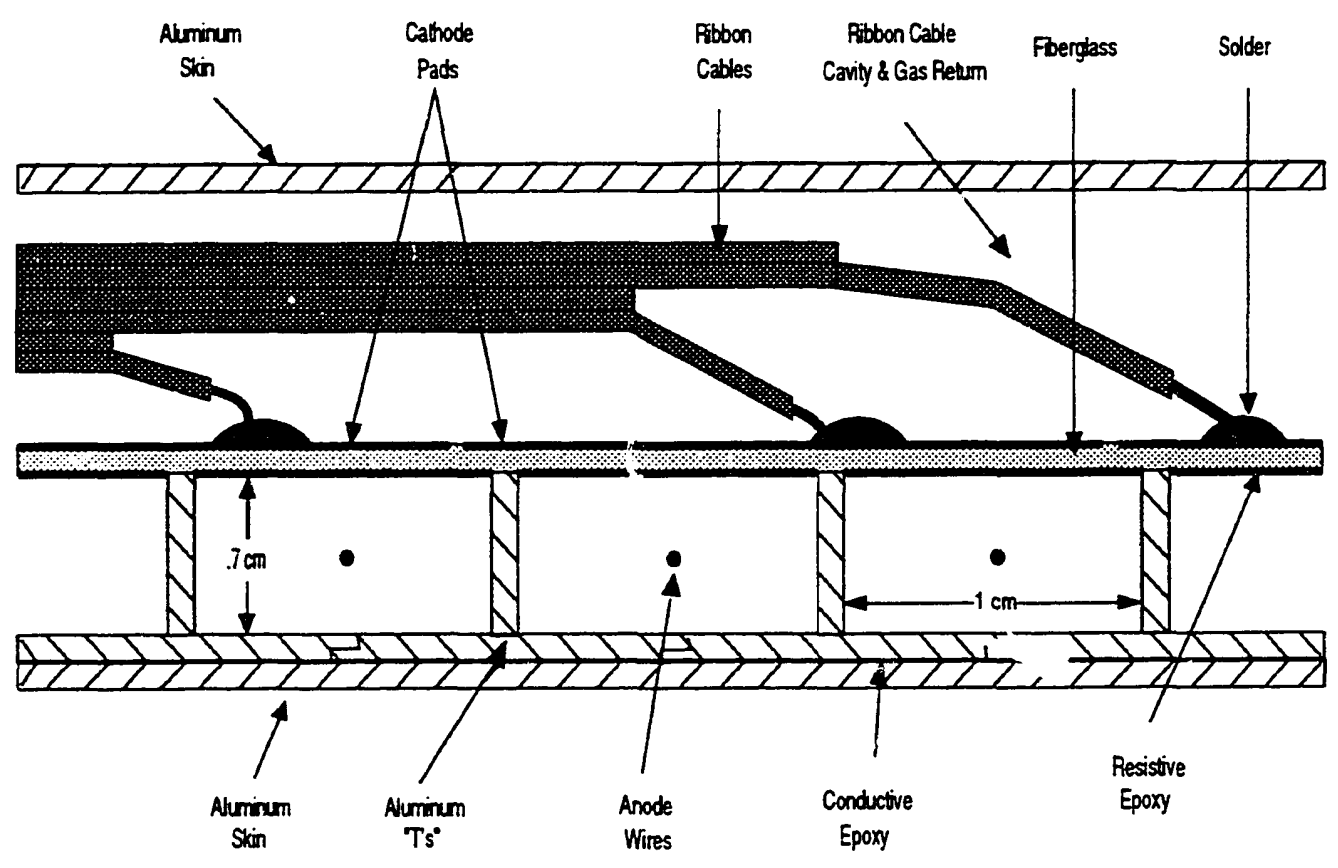

Figure 4.13: Cross section of a typical FEM chamber.

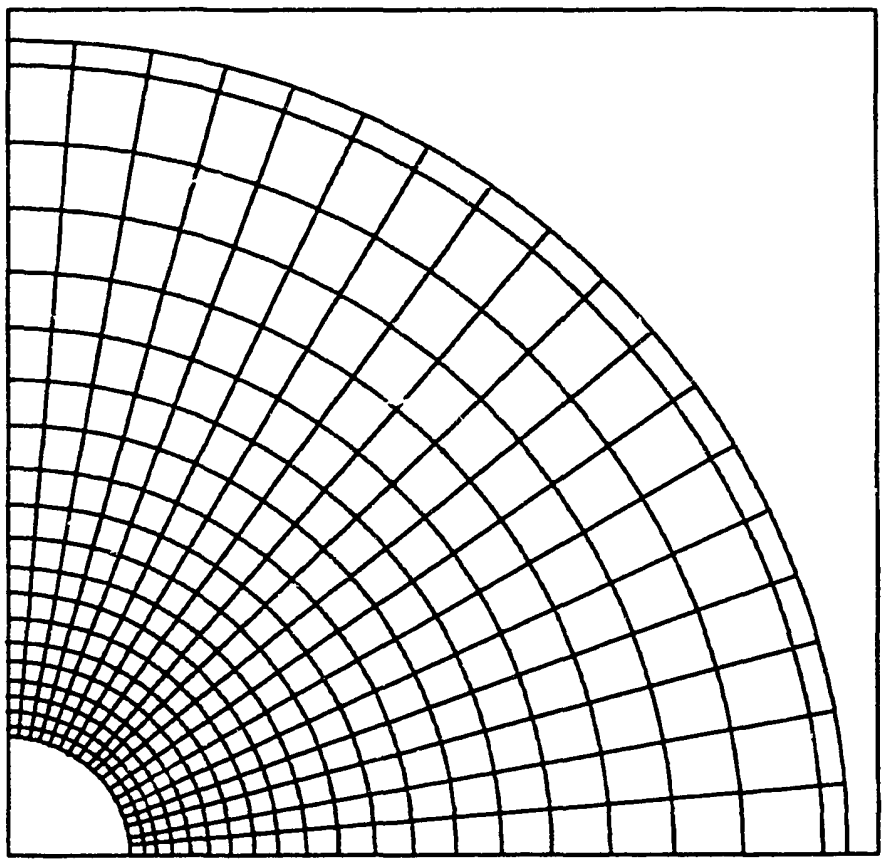

Figure 4.14: Pad layout of FEM layer 10. 


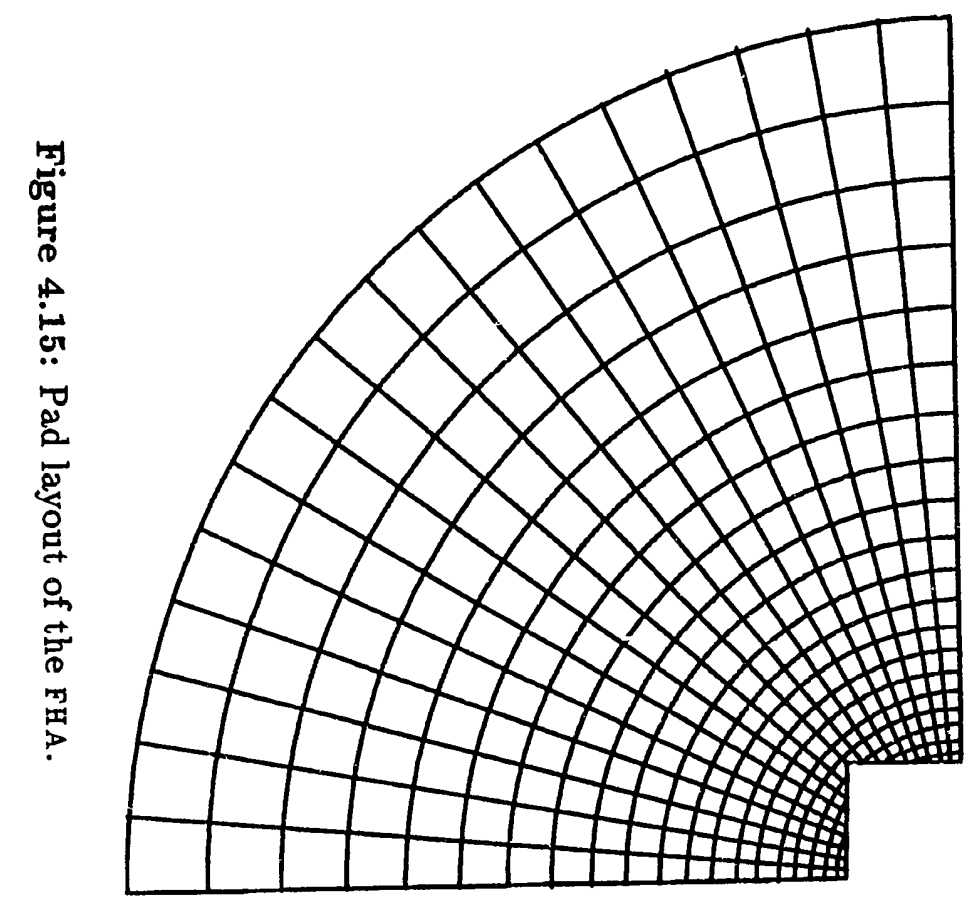


It can be seen in figure 4.14 and figure 4.15 that the calorimeter has a hole cut out of the center of it to make room for the low-beta quads. The calorimeter does not, therefore, cover the complete $2 \pi$ range in $\phi$ all the way to $\eta=4.2$. The calorimeter is hermetic out to $\eta=3.6$.

Table 4.5 summarizes the specification of the forward calorimeters.

\subsection{Central Muon Chambers}

Muons will leave a track in the tracking chambers but are too massive to shower in the calorimeters. The central muon chambers (CMU) are positioned on the outside of the central calorimeter wedges; see figure 4.16. The CMU is basically a tracking chamber; the assumption being that any particle that can make it through the 4.9 absorption lengths that precede the CMU is most probably a muon. Muon identification will be described in detail in a later section.

The CMU is constructed from rectangular streamer chambers (drift tubes) 226 $\mathrm{cm}$ long; figure 4.17 shows the cross section of one of the drift tubes. Each module of the CMU consists of 4 layers of 4 drift tubes. Three of these modules are combined on each wedge. The $\phi$ coverage of the CMU is $12.6^{\circ}$ out of each of the $15^{\circ}$ wedges, and the $\eta$ coverage is from 0.03 to 0.63 .

A charged particle passing though one of the drift tubes will leave an ionization trail that then drifts to the sense wire. A TDC on each wire records the arrival time of the ionization. The sense wires are offset in two of the four layers, therefore it is possible to unambiguously determine the path of the muon through the chamber (in the $\mathrm{r}-\phi$ plane); figure 4.18 shows a cross section of one of these muon towers.

In addition to the TDC readout for each wire, a charge division measurement is also recorded. By comparing the amount of charge arriving at both ends of the sense wire it is possible to determine the $z$ position of the track.

Table 4.6 summarizes the specifications of the central muon chambers.

\section{Forward Muon Chambers}

For completeness it should be mentioned that CDF has a muon detection system behind the forward calorimeters as well (FMU). These muon detectors are not used in this analysis. 


\begin{tabular}{||l|c|c||}
\hline & FEM & FHA \\
\hline Coverage $(|\eta|):$ & $2.2-4.2$ & $2.3-4.2$ \\
Tower Size $(\Delta \eta \times \Delta \phi)$ & $0.1 \times 5^{\circ}$ & $0.1 \times 5^{\circ}$ \\
\hline Active Medium & \multicolumn{2}{|c||}{$50 / 50$ Argon-ethane proportional } \\
& tube chambers with cathode pad readout \\
\cline { 2 - 3 } Thickness & $0.7 \mathrm{~cm}$ & $1.0 \mathrm{~cm}$ \\
Layers & 30 & 27 \\
Absorber & $\mathrm{Pb}$ & $\mathrm{Fe}$ \\
Thickness & $0.48 \mathrm{~cm}$ & $5.1 \mathrm{~cm}$ \\
Layers & 30 & 27 \\
\hline Energy Resolution & & $\frac{130 \%}{\sqrt{E}} \oplus 4 \%$ \\
$\left(\frac{\sigma}{E(G e V)}\right)$ & $\frac{25 \%}{\sqrt{E}} \oplus 0.5 \%$ &
\end{tabular}

Table 4.5: Forward Calorimeter Specifications

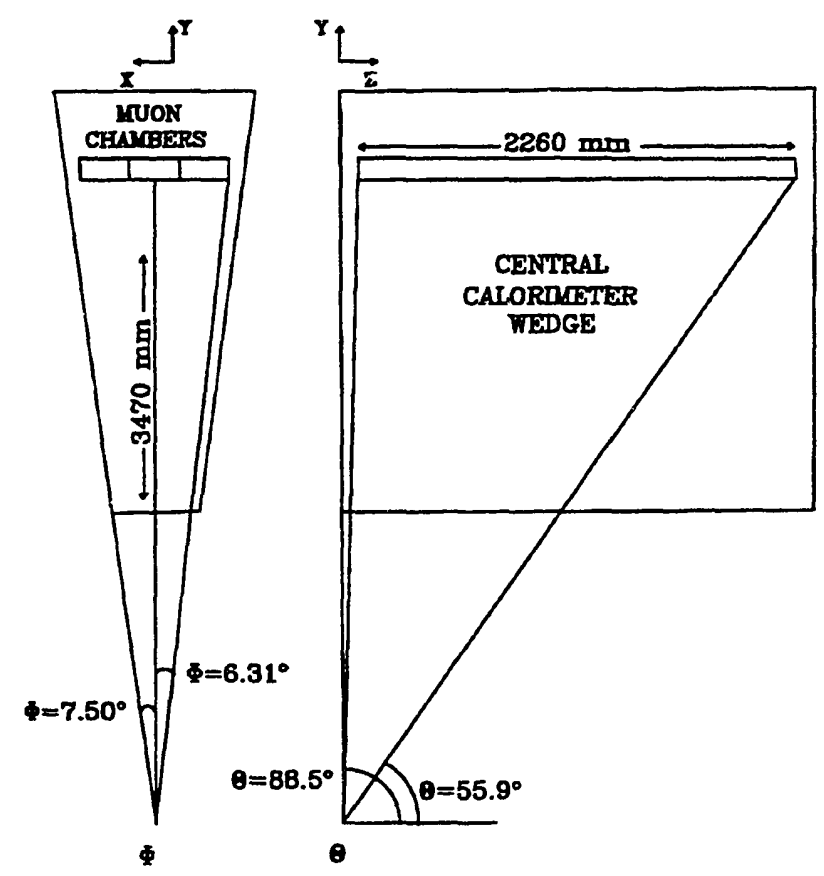

Figure 4.16: Location of the $\mathrm{CMU}$ chambers within the central calorimeter. 


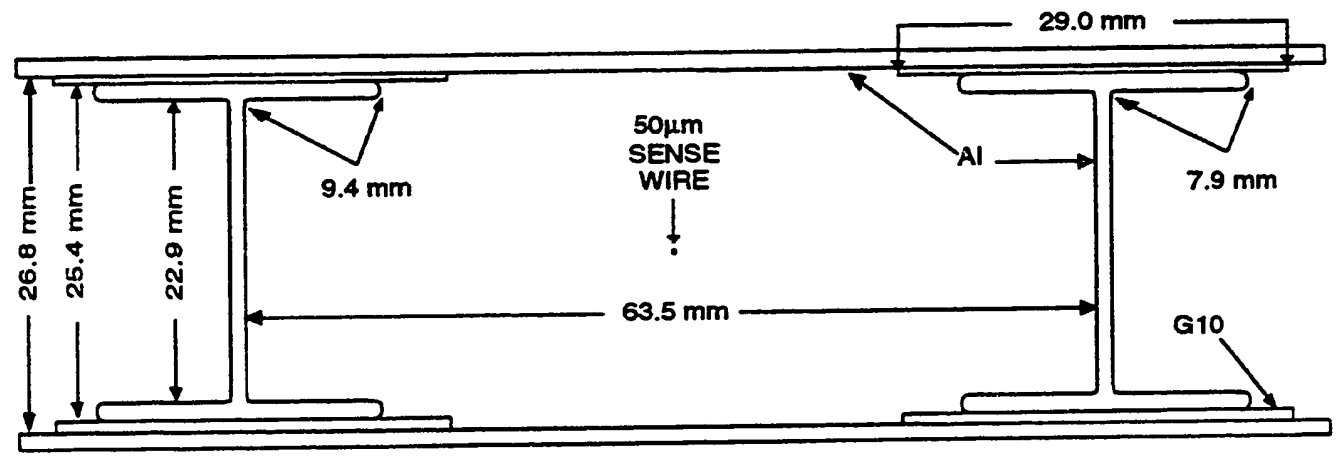

Figure 4.17: CMU drift cell.

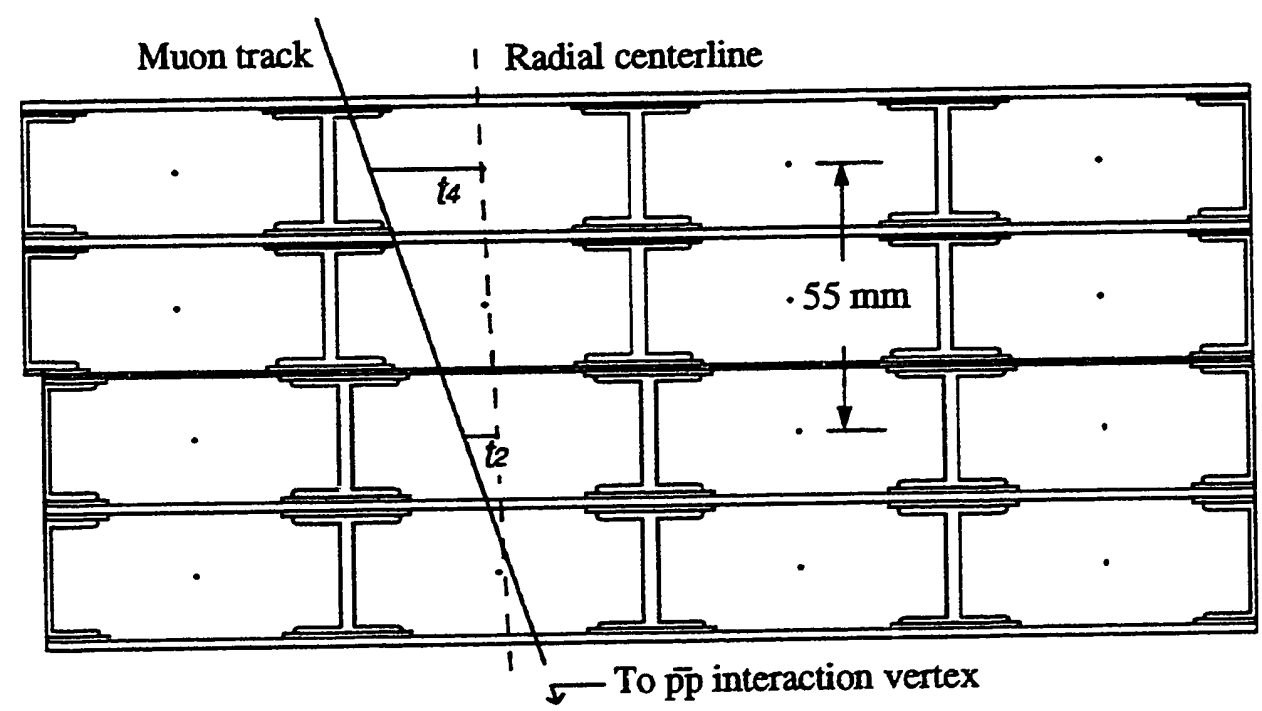

Figure 4.18: A CMU tower. A hypothetical muon track is drawn through the 4 layers. 


\begin{tabular}{||lc||}
\hline Angular coverage & $55.9^{\circ}<\theta<124.1^{\circ}$ \\
& $-0.63<\eta<0.63$ \\
\hline Modules per wedge & 3 \\
Layers & 4 \\
Drift tubes per module & 16 \\
Drift tube length & $226.1 \mathrm{~cm}$ \\
Drift tube width & $6.35 \mathrm{~cm}$ \\
Drift tube height & $2.68 \mathrm{~cm}$ \\
Gas & $50 / 50$ argon-ethane \\
Distance from beam & $347 \mathrm{~cm}$ \\
\hline Resolution: & \\
Drift direction & $250 \mu \mathrm{m}$ \\
Z (charge division) & $1.2 \mathrm{~mm}$ \\
\hline
\end{tabular}

Table 4.6: Central Muon Chamber (CMU) Specifications 


\subsection{Data Acquisition System}

The Tevatron produces roughly 50,000 collisions per second; however, the CDF data is stored on magnetic tapes at a maximum rate of about 2 events per second. In order to accomplish this event rate reduction, CDF has a programmable triggering system to filter out only those events that are of interest. The trigger can look for events with high $\mathrm{P}_{t}$ electrons, photons, muons, jets, tracks, missing transverse momentum, or various combinations of these objects.

Once a decision has been made to keep an event a full readout of the detector is initiated; the process of reading out the entire detector takes approximately $10 \mathrm{mS}$. In order to reduce the dead-time involved in making a trigger decision, each of the detector components is equipped with a "fast-out" signal. This fastout signal is used by a multi-level triggering system to reduce the $50 \mathrm{kHz}$ rate of the Tevatron down to approximately $100 \mathrm{~Hz}$, so that detector readout does not impose a large dead-time. The CDF trigger has 4 levels (0-3); below is a description of the information available at each level and a description of the electron (and muon) triggers used to gather the $\mathrm{W}$ boson candidates for this analysis (there were many other triggers running in parallel with the lepton triggers, but they are not particularly relevant to this analysis).

\section{Level 0 Trigger}

A plane of scintillation counters is installed on the front face of each of the forward calorimeters. These scintillators-called the beam-beam counters (BBC)are used to provide a "minimum-bias" trigger. The scintillators have excellent timing characteristics ( $\sigma<200 \mathrm{ps}$ ), and the level 0 trigger simply requires hits in coincidence in the two BBC's (within 100 ns of the expected beam crossing time). The decision time for level 0 is less than the $3.5 \mu \mathrm{S}$ interval between beam crossings and so did not introduce any dead-time.

\section{Level 1 Trigger}

If the level 0 trigger is satisfied then the level 1 trigger makes its decision. The following information is available to the level 1 trigger. The calorimeter information is ganged into "trigger towers" each of which is 0.2 units wide in $\eta$ and $15^{\circ}$ wide in $\phi$. The central muon chamber readout is also available to level 1 . This analysis uses a trigger designed to find high $\mathrm{Pt}$ leptons. For the case of electrons the level 1 requirement was a trigger tower with at least $6 \mathrm{GeV}$ of transverse electromagnetic energy. For the muon case the requirement was that there be hits in coincidence in at least 2 of the 4 layers in a given tower, and that the timing be consistent with a muon with at least $3 \mathrm{GeV} \mathrm{P}_{t}$. 
On average, one beam crossing was missed while the level 1 trigger decision was being made. The level 1 triggers can be prescaled (accepting only a fraction of the events satisfying the trigger requirements) so that high-rate events (e.g. low $\mathrm{P}_{t}$ dijets) could be accepted along with low-rate events (e.g. W events). The level 1 trigger was tuned to accept events at a rate of a few $\mathrm{kHz}$.

\section{Level 2 Trigger}

Events passing the level 1 trigger were then presented to the level 2 trigger. The level 2 trigger has a hardware cluster finder to search for clusters of energy in the calorimeters. There also exists a hardware track processor that can identify tracks in the CTC with $\mathrm{P}_{t}>6 \mathrm{GeV}$ and very roughly measure their $\mathrm{P}_{t}$; this information is also presented to the level 2 trigger. The level 2 trigger can also match the calorimeter clusters with tracks from the fast CTC hardware track finder. The level 2 requirement for electrons is a cluster in the central calorimeter with $\mathrm{P}_{t}>$ $12 \mathrm{GeV}$ matched -in $\phi$ - to a track with $\mathrm{P}_{t}>6 \mathrm{GeV}$. The cluster is also required to have a ratio of hadronic energy to electromagnetic energy of less than 0.125 . The requirement for muons was a level 1 muon matched to a track with $\mathrm{P}_{t}>9.2$ $\mathrm{GeV}$.

The level 2 decision was made in $20 \mathrm{uS}$, and it's acceptance rate was tuned to be 3-4 Hz. A level 2 trigger accept initiated a full detector readout.

\section{Level 3 Trigger}

The final trigger decision was made by a bank of 60 independent processors running FORTRAN event reconstruction code (very similar to the offline reconstruction code). The level 3 trigger had access to the full detector readout. The level 3 requirement for electrons was the same as the level 2 requirement. The level 3 requirement for muons is a muon stub with a matching CTC track with $\mathrm{P}_{t}>11$ $\mathrm{GeV}$.

The level 3 triggers were tuned to provide a $1-2 \mathrm{~Hz}$ acceptance rate. 


\section{Chapter 5}

\section{Event Reconstruction and Selection}

This chapter describes the offline reconstruction of electrons, muons, jets, and missing $E_{t}$. Each of these objects leaves a very distinct signature in the CDF detector, and cuts are described which are designed to separate these objects from backgrounds that can fake similar signatures.

\subsection{Electrons}

Electrons will manifest themselves in the detector as a cluster of electromagnetic energy whose characteristics are consistent with testbeam electrons. They will also leave a CTC track whose momentum matches the energy measured in the calorimeter.

The following sections describe how the EM clusters are found and the cuts that are applied to them in order to remove as much non-electron background as possible (without being too inefficient at identifying real electrons). The response of the CDF detector to electrons varies across the face of the detector and the following sections describe the energy/position corrections that are applied to the electron candidate.

\subsubsection{Electron Clustering}

The first step in identifying electrons is to search the electromagnetic calorimeters for energy "clusters". First, a list of seed towers is made from all towers with at least $3 \mathrm{eV}$ of $\mathrm{E}_{t}$. Electron showers are approximately the size of one tower in the central calorimeter, therefore they should only deposit energy in at most 3 towers in $\eta$. Electron showers do not extend across the $\phi$ boundaries of the CEM because 
there is a lot of material in the regions between the wedges. Towers adjacent to the seed towers (in $\eta$ ) are added to the cluster if they have $\mathrm{E}_{t}>0.1 \mathrm{GeV}$. If an adjacent tower has more energy than the seed tower it becomes the seed tower and the clustering is started over again on that tower. Finally, clusters were kept only if the sum of the $E_{t}$ 's of all towers in the cluster was at least $5 \mathrm{GeV}$, and the ratio of the hadronic to electromagnetic energy (in the cluster's towers) was less than $12.5 \%$.

\subsubsection{Electron Identification Cuts}

Electrons from $\mathrm{W}$ decays should have a large transverse momentum and be quite isolated from any other energy in the event. The following cuts were applied to the electron candidates (the energy clusters described above) in order to retain only electrons from W's (and Z's, which will be removed later). See figures 5.1 and 5.2 for the distribution of each of the quantities cut on.

1. The electron was required to be in the fiducial regions of the central electromagnetic calorimeter. The last tower on each side of the CEM is excluded, which confines the electron to the region $-1.0<\eta<1.0$ (where $\eta$ is from $z=0$, not the event vertex). The position of the electron within the tower was obtained using the information from the central electron strips (CES). The electron was required to be within $21 \mathrm{~cm}$-in the $r \phi$ direction-of the center of the tower (this prevents electrons from going into the region between the wedges). Furthermore, the electron was required to be at least $9 \mathrm{~cm}$ away from the $\eta=0$ crack in the detector. One of the CEM wedges has a hole in it for the magnet cryogenics; the electron was not allowed to be in the range $0.82<\eta<0.92$ in that wedge. Finally, the electron was excluded from a short list of towers that were known to have hardware problems.

2. The electron is required to have $|\eta|<0.95$. This $\eta$ is measured from the $z$ vertex of the event (not necessarily $z=0$ ). The cut represents the point at which the acceptance from the fiducial cuts, described above, drops below about $50 \%$. Figure 5.4 shows the electron acceptance as a function of $\eta$. Corrections for this acceptance effect are discussed in section 5.1.4.

3. After all corrections the electron was required to have $\mathrm{P}_{t}>20 \mathrm{GeV}$. The corrections applied to the electron are described in the next section.

4. Electrons should deposit most of their energy in the electromagnetic calorimeter. The following cut made on the ratio of the hadronic to the electromagnetic energy in the cluster:

$$
\frac{\text { Hadronic }}{E M}<0.055+0.045 * \frac{E_{\text {clus }}}{100},
$$



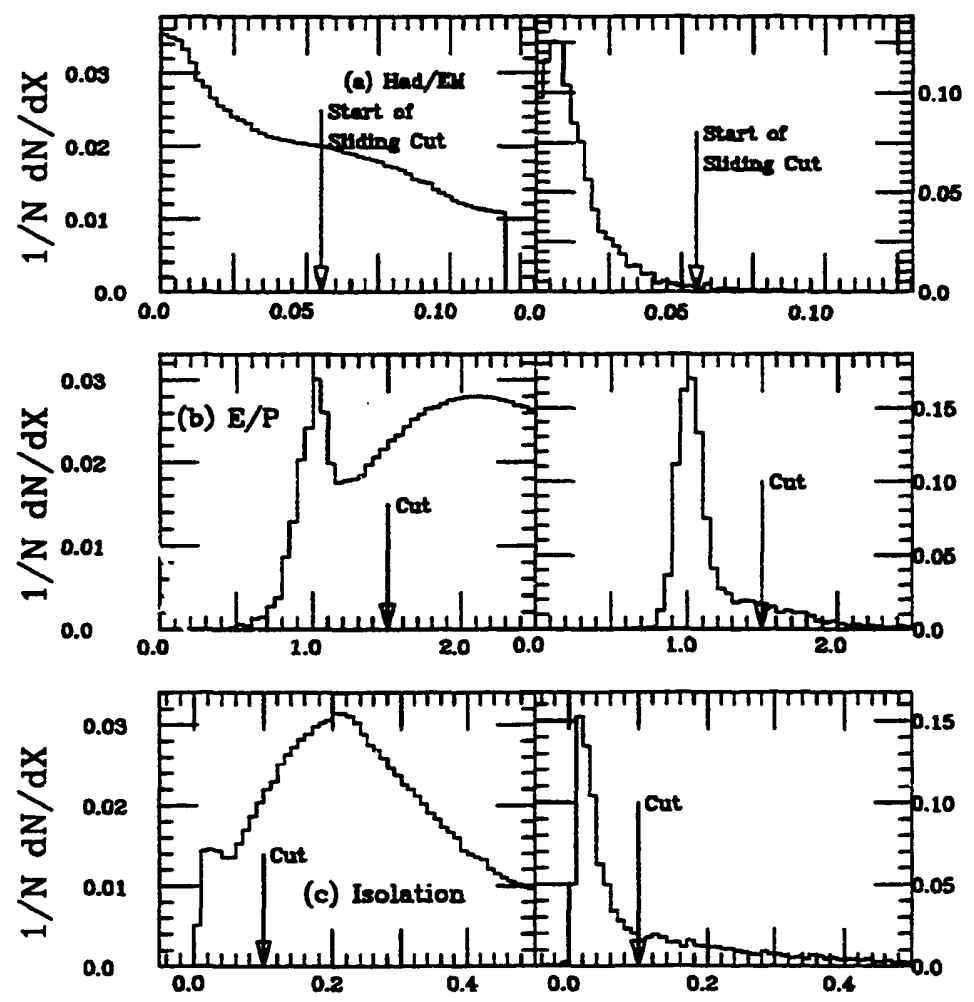

Figure 5.1: Distributions of the electron identification cuts $\mathrm{Had} / \mathrm{EM}, \mathrm{E} / \mathrm{P}$, and the Isolation. The plot on the left shows the distribution without any of the other ID cuts applied, except for the $20 \mathrm{GeV} P_{t}$ cut and those cuts intrinsic to the trigger. The plot on the right shows the distribution with all other ID cuts applied. This figure was taken from [16]. 

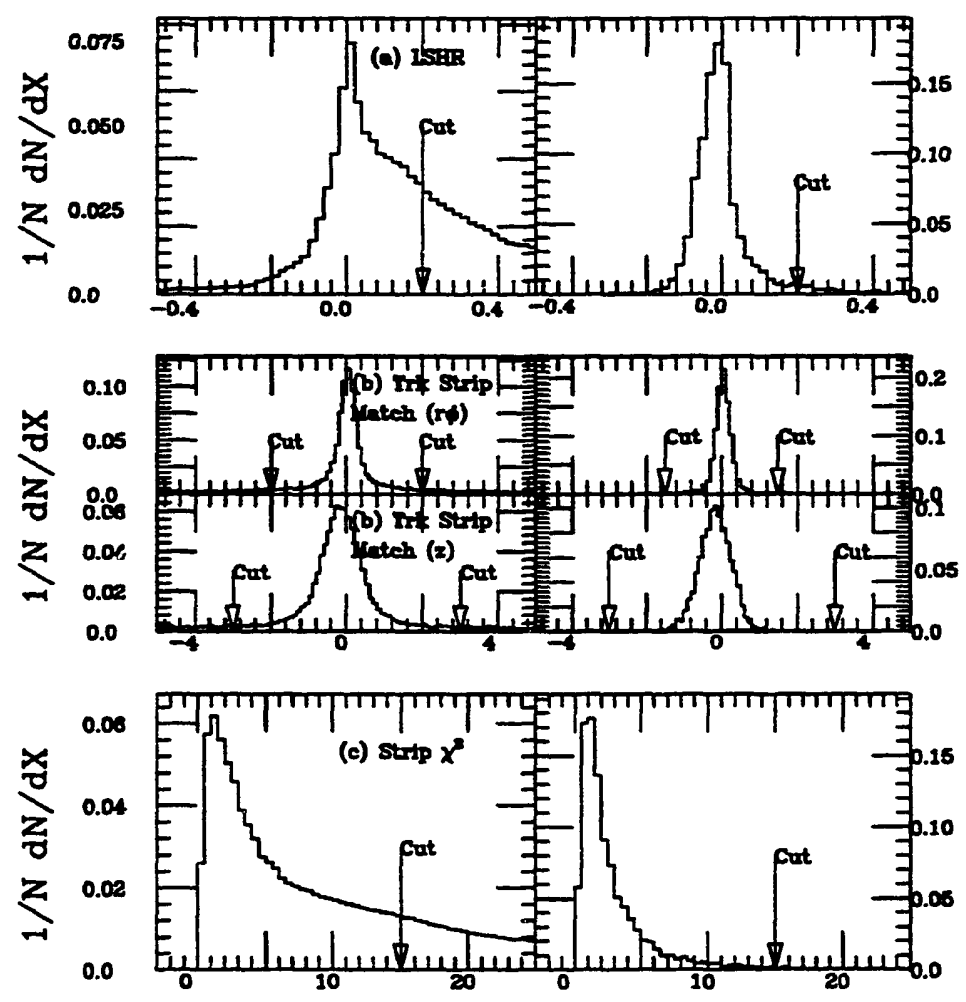

Figure 5.2: Same as figure 5.1 for the electron id cuts LSHR, $r \phi$ track match, $\mathrm{Z}$ track match, and strip chamber $\chi^{2}$. This figure was taken from [16]. 
where $E_{\text {clus }}$ is the uncorrected EM energy of the electron cluster. Higher energy electrons tend to leak more of their energy into the hadronic compartment of the calorimeter; the energy dependance of the Had/EM cut takes this into account so that the efficiency of the cut is not energy dependent.

5. The transverse shape of the electron's signal in the CES must match the shape measured using testbeam electrons. The profile of the electron signal in the strips is compared to the standard electron profile and a $\chi^{2}$ is computed. The $\chi^{2}$ is required to be less than 15 (the exact definition of this variable requires much detail to explain, see [13] for a detailed description).

6. The transverse shape of the electron's energy deposition in the CEM towers is also compared to that expected from testbeam electrons. The quantity LSHR is defined as:

$$
L S H R=A \sum_{i} \frac{F_{i}-T_{i}}{\sigma_{i}},
$$

where $i$ runs over all towers in the cluster, $F_{i}$ is the ratio of the energy in the tower to the energy in the seed tower, $T_{i}$ is the same ratio measured for testbeam electrons, $\sigma_{i}$ is the standard deviation of the testbeam measurement, and $\mathrm{A}$ is a normalization constant $(\mathrm{A}=0.14)$. Electrons are expected to have a very narrow shower and, therefore, small values of LSHR. The electron is required to have LSHR $<0.2$.

7. Electrons from W's should be isolated from other energy in the detector (electrons from b quark decays, as a counter example, will be very close to the energy deposits from the other $b$ decay products). The "isolation" of the electron is defined as:

$$
I=\frac{E_{t}^{0.4}-\mathrm{E}_{t}}{\mathrm{E}_{t}},
$$

where $E_{t}$ is the uncorrected transverse energy of the electron cluster, and $E_{t}^{0.4}$ is the sum of the transverse energy in all towers within a cone of radius 0.4 , in $\eta-\phi$ space, centered on the electron. The isolation is required to be less than 0.1 .

8. The electron is required to have left a track in the CTC. The track is constrained to come from the known $x-y$ position of the beam (which improves the momentum and direction resolution of the track); and is then required to meet the following requirements: 
(a) The momentum of the track- $\mathrm{P}$-and the energy of the electron cluster (after energy corrections)-E-must satisfy:

$$
\frac{E}{P}<1.5 \text {. }
$$

The cut is set at 1.5 to allow for some photon radiation from the electron.

(b) When the track is extrapolated through the CDF detector to where it intersects the CES, it must match the position of the CES cluster within $1.5 \mathrm{~cm}$ in the $r \phi$ direction and within $3.0 \mathrm{~cm}$ in the $z$ direction.

(c) The point of closest approach of the track to the beamline must within $60 \mathrm{~cm}$, in $z$, of the center of the detector.

\subsubsection{Electron Corrections}

The response of the CDF detector is not completely uniform over the fiducial region allowed for electrons. Two different corrections are made in order to remove these non-uniform effects.

Although the cuts on the electrons keep them away from the edges of the CEM towers, the CEM response varies over the allowed region. This response was measured in the testbeam and is shown in figure 5.3. The response was measured for each of the nine towers in $\eta$. The electron energy is corrected for this effect. These response maps are normalized so that the value is 1.0 at the center of each tower.

There are small variations in materials, and electronics for the readout, therefore the response of every tower in the CEM will be slightly different. The relative response of each tower was measured by looking at the average E/P (where $P$ is the track momentum) for the entire sample of electron triggers taken during the collider run. The CTC was calibrated by carefully studying the magnetic field inside the tracking chamber and by studying the tracks from $\psi \rightarrow \mu^{+} \mu^{-}$and $\Upsilon \rightarrow \mu^{+} \mu^{-}$decays.

The electron energy was typically changed by less than $4 \%$ by the two corrections described above.

The full electron 4-vector was defined as follows. The electron energy is the energy of the corrected electron cluster. The direction of the electron is taken as the direction of the track at the point of closest approach to the beamline (this removes the small change in the electron direction incurred as it traveled through the magnetic field). The absolute value of the momentum part of the 4-vector was normalized to give the electron a zero mass (the true mass of the electron is much smaller that the detector's mass resolution). 


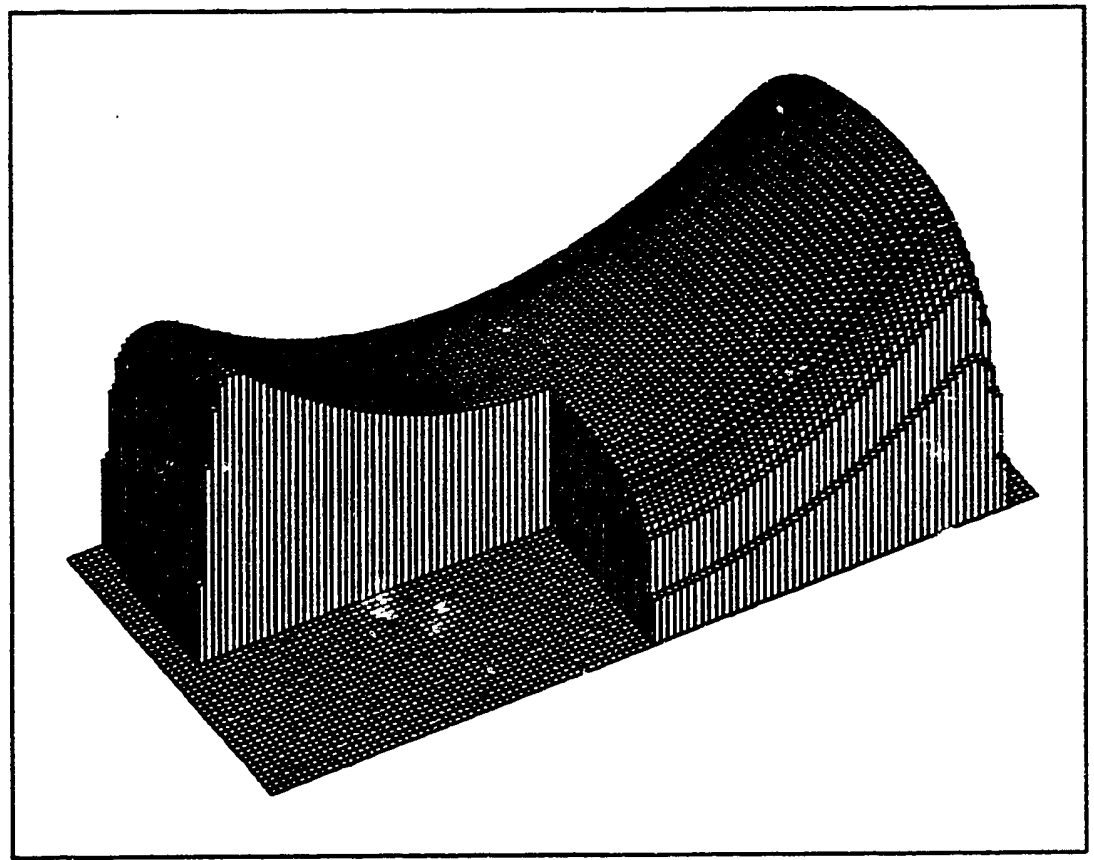

Figure 5.3: The response of a typical CEM tower as measured over the face of the tower. The lower left section has been cut out for clarity. The map is normalized to be 1.0 in the center of the tower. The lowest value shown is 0.9 . The shorter axis is $z$ (the direction along the beamline), and the longer axis is $\mathrm{x}$ (the $r-\phi$ direction). 


\subsubsection{Electron Eta Acceptance Correction}

Figure 5.4 shows the electron acceptance as a function of $\eta_{\text {Lab }}$. The shape of the acceptance is caused by the fiducial cuts that were applied to the electron. The variations in the acceptance, within the allowed region $\left(\left|\eta_{\mathrm{Lab}}\right|<0.95\right)$, are corrected for by weighting the events with the inverse of the acceptance. This acceptance correction was not applied in the excited quark searck: 'which is described in Chapter 11).

\subsection{Muons}

The muonic decays of the $\mathrm{W}$ are not used in the measurement of the production angle of the W; however, they are used in Chapter 11 to search for excited quark states decaying into a $\mathrm{W}$ and a jet, so their reconstruction is described in this section.

Muons leave a very distinctive signature in the detector. A CTC track pointing to hits in the muon chambers (CMU) with very little energy deposited in the traversed calorimeter tower.

Muon reconstruction starts by looking for track stubs in the muon chambers. For each siub found the following requirements are made:

1. The track associated with the muon must have $P_{t}>18 \mathrm{GeV}$ before being constrained to the known beam position in the $x-y$ plane. The track is then constrained to come from an $x-y$ position consistent with the known beam position and the track $\mathrm{P}_{t}$ was then required to be greater than 20 $\mathrm{GeV}$.

2. The muon trajectory is then extrapolated, from the point where it left the CTC, through the detector until it hits the muon chambers. This projected point is required to be in the fiducial region of the CMU chambers. The muon must satisfy $0.04<|\eta|<0.61$ (where $\eta$ is determined from $z=$ 0 ). The muon must lie within $6^{\circ}$, in $\phi$, of the center of the wedge (see figure 4.16). The muon must not have $\eta>0.5$ in the wedge pierced by the superconducting magnet cryogenics plumbing. Finally the muon was excluded from chambers that were known to have some kind of hardware problem (for most of the run this was only one chamber).

3. The muon is required to have $|\eta|<0.6$. This $\eta$ is measured from the $z$ vertex of the event (not necessarily $z=0$ ). The cut represents the point at which the acceptance from the fiducial cuts, described above, drops below about $50 \%$. 


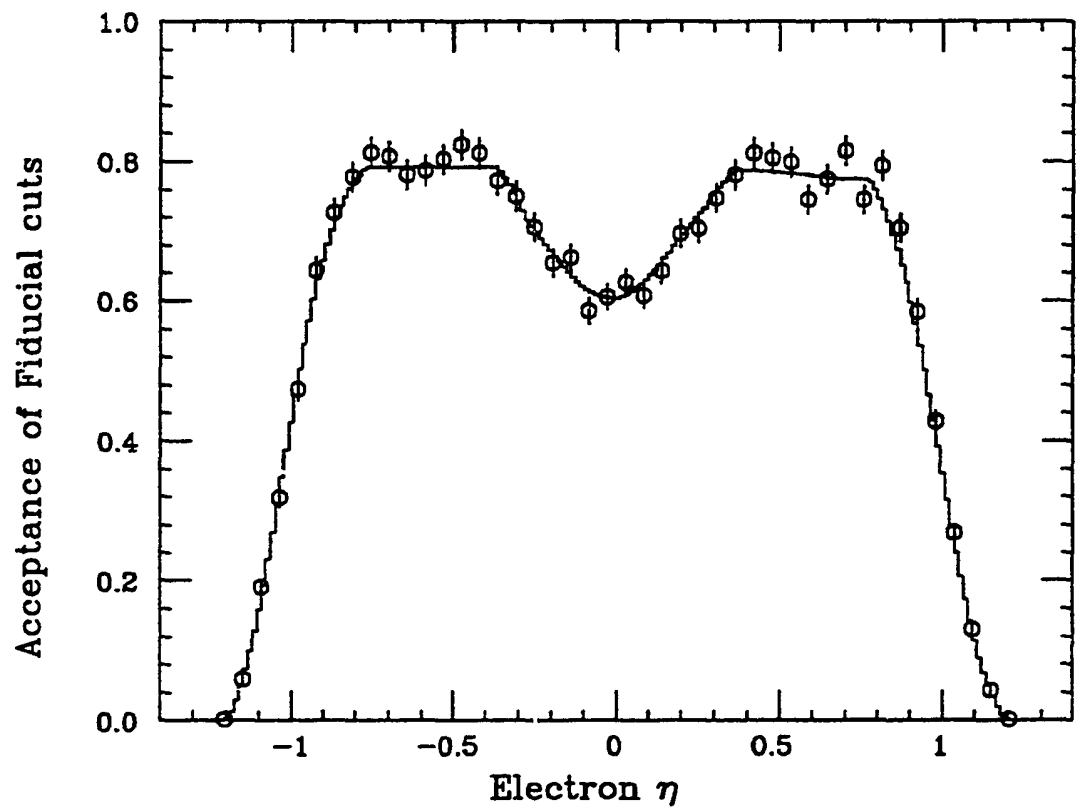

Figure 5.4: Electron acceptance as a function of pseudo-rapidity. The points are the result of using a detector simulation, and the line is what is expected from the fiducial cuts. 
4. The point of closest approach of the track to the beamline was required to have a $z$ value within $5 \mathrm{~cm}$ of an event vertex (as measured in the VTPC) with $|z|<60.0 \mathrm{~cm}$.

5. The central calorimeter tower traversed by the muon should only measure enerzy consistent with the passage of a minimum-ionizing particle. The electromagnetic tower was required to have less than $2.0 \mathrm{GeV}$ of energy in it and the hadronic tower less than $6.0 \mathrm{GeV}$. This cut also removes events in which the muon is not isolated from other energy in the event (e.g. the muon is part of a hadronic jet).

6. The projected intercept point for the track is required to match the position of the muon chamber track. The two tracks were required to be within $2.0 \mathrm{~cm}$ of each other in the $r \phi$ direction.

7. Muons from the decay of the $\mathrm{W}$ boson should be isolated from the other energy in the event. Muons that are near other energy in the event are more likely to have come from inside a jet or are not really muons but particles coming out the back of the central hadronic calorimeter as the result of a jet (the process known as "punch through"). The isolation of the muon is defined as:

$$
I=\frac{\mathrm{E}_{t}^{0.4}-E_{\text {tower }}}{\mathrm{P}_{t}^{\text {track }}},
$$

where $E_{t}^{0.4}$ is the total $E_{t}$ in a cone of radius 0.4 around the muon, $E_{\text {tower }}$ is the energy in the tower traversed by the muon, and $P_{t}{ }^{t r a c k}$ is the $P_{t}$ of the associated track. The isolation was required to be less than 0.1 .

The momentum of the muon was defined as that of the beam-constrained track and the energy of the muon was set to give the muon a zero mass (the mass of the muon is small compared to the tracking resolution).

\subsection{Jets}

High $\mathrm{P}_{t}$ quarks and gluons produced in the collision result in a spray, or "jet", of hadrons (see section 2.3.3). These jets will show up as clusters of energy in the calorimeters. CDF uses a fixed-cone (in $\eta-\phi$ space) clustering algorithm to find jets [17].

The jet clustering algorithm begins by choosing seed towers as any tower with $\mathrm{E}_{t}>1 \mathrm{GeV}$ (electromagnetic $\mathrm{E}_{t}+$ hadronic $\mathrm{E}_{t}$ ). When choosing these seeds the towers in the plug and forward regions were grouped into $\Delta \phi=15^{\circ}$ "towers" in order to match the central segmentation (this prevents any bias by uniformly segmenting the detector). 
The next step was to "pre-cluster" these seed towers. Seed towers were combined if they touched on a side or a corner, and they were required to fall monotonically in energy going out from the highest energy tower in the pre-cluster. The $\mathrm{E}_{t}$-weighted centroid (in $\eta-\phi$ space) of the pre-cluster was used as the starting point for the fixed-cone clustering.

The full CDF segmentation was used for the fixed-cone clustering. All towers with $\mathrm{E}_{t}>100 \mathrm{MeV}$ and inside a circle of radius 0.7 were associated with the cluster. The cluster's $\mathrm{E}_{t}$-weighted centroid was re-calculated using all of the towers in the cluster. This process was iterated until the towers associated with the cluster remained unchanged. The uriginal seed towers were always included in order to keep the clusters from wandering too far from their original starting point.

Each cluster was iterated independently; therefore, some clusters may have towers in common. To distribute the shared towers between two clusters the $\mathrm{E}_{t}$ of all the shared towers were added together. If the common towers accounted for more than half the total $E_{t}$ of either of the two clusters then the two clusters were merged together into one cluster; otherwise the two clusters were separated. To separate the two clusters, the shared towers were assigned to the nearest (in $\eta-\phi$ ) cluster. The centroids were re-calculated and the original set of shared towers was again distributed to the closest cluster. This procedure was iterated until the distribution of the towers was unchanged.

The momentum of the jet was determined by adding together each tower in the cluster as if it were a zero-mass particle. For this analysis, the energy of the jet was then chosen so that the mass of the jet was zero. Zeroing the jet mass was done for two reasons. It is difficult to apply corrections to the measured jet mass, and, as mentioned in section 2.3.3; the leading order theories do not predict the jet mass. Choosing to use the jet mass or not to use it has little effect on the results of this analysis.

\subsubsection{Jet Corrections}

The response of the CDF detector to jets is a non-linear function of the jet's $\mathrm{P}_{t}$, because the detector's response to low energy hadrons is non-linear. The response to low energy hadrons was measured in the testbeam, and by studying low $\mathrm{P}_{t}$ tracks in minimum-bias events (events that are only required to pass the level 0 trigger, see section 4.7). Figure 5.5 shows the response of the central calorimeter to low energy hadrons. A detector simulation, which included the measured response to low energy hadrons, was then used to develop jet correction factors as a function of the jet's $P_{t}$. The jet corrections take the $P_{t}$ measured in the cluster and attempt to correct back to the $\mathrm{P}_{t}$ of all particles hitting the calorimeter within the 0.7 radius cone of the cluster.

The response of the CDF detector to jets is also a function of $\eta$-due to the large 


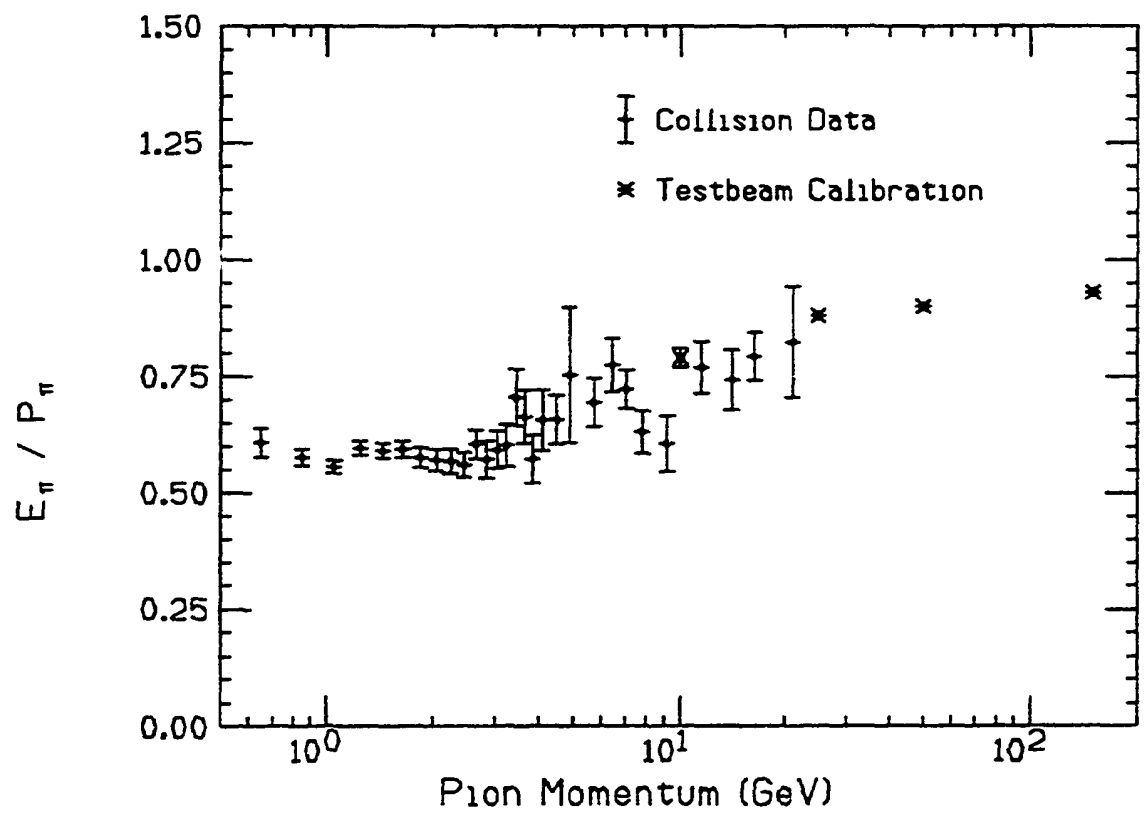

Figure 5.5: The response of the central calorimeter to low energy charged pions. Measurements made in the testbeam are shown, as well as the in situ measurements made using minimum-bias events. 
crack regions between the central, plug, and forward calorimeters. The relative response of the detector, as a function of $\eta$, was measured using a sample of events collected with a single jet trigger. Events with two jets were used, and one jet was required to be well within the central region of the detector $(0.2<|\eta|<0.7)$. The scalar sum of the two jets' $P_{t}$ was required to be more than 2.0 times the single-jet trigger threshold (to remove any bias introduced by triggering on events in which the jet energy measurement fluctuated high). The second jet was then used to probe the response of the detector as a function of $\eta$ (because any $\eta$ dependance of the $\mathrm{P}_{t}$ of the second jet should be due to the detector). Figure 5.6 shows the correction function applied to the jets, as a function of $\eta$, for two different measured jet $P_{t}$ 's (the boundary regions between the detectors are very clear in this plot). In this way an $\eta-P_{t}$ response map for jets was determined.

One final correction is applied to the jet clusters. The underlying event tends to deposit energy uniformly in $\eta-\phi$ space. By studying the energy flow in minimumbias events it can be seen that the underlying event deposits, on average, 1.13 $\mathrm{GeV}$ of $\mathrm{P}_{t}$ in a 0.7 cone. This is subtracted from the jet $\mathrm{P}_{t}$.

\subsection{Missing $\mathrm{E}_{t}$}

The neutrino, from the $\mathrm{W}$ decay, will pass through the detector without leaving any measurable signal; however, neutrino production can be detected by looking for an imbalance in the transverse momentum measured in the detector. CDF has nearly hermetic coverage in the region $-3.6<\eta<3.6$. Many particles from the collision will leave the detector beyond this value in $\eta$; however, at these very small angles the particles cannot carry away much transverse momentum (e.g. the ratio of momentum to transverse momentum at $\eta=3.6$ is 18.3). Note that the low angle particles can carry away significant momentum in the $\mathrm{z}$ direction and so the $z$ component of the neutrino's momentum is not "measurable".

The missing transverse momentum in an event is estimated by the "missing transverse energy" vector $\left(\overrightarrow{\mathrm{E}}_{t}\right)$, defined as:

$$
\overrightarrow{\mathrm{E}}_{t}=-\sum_{i} E_{i} \sin \theta_{i} \hat{n}_{i}
$$

where $i$ runs over all calorimeter towers with $|\eta|<3.6 ; E_{i}$ is the energy in the tower, $\theta_{i}$ is the angle between the $z$ axis and the line from the event vertex to the tower, and $\hat{n}_{i}$ is the unit vector in the transverse plane pointing to the tower. The sum includes only those towers with $E_{i} \sin \theta_{i}>100 \mathrm{MeV} . \overrightarrow{\mathrm{H}}_{t}$ approximates the missing transverse momentum; it assumes that the mass of the energy deposited in each tower is zero-which may not be true, especially if two or more particles hit that tower. This assumption is why the quantity has been named the missing 


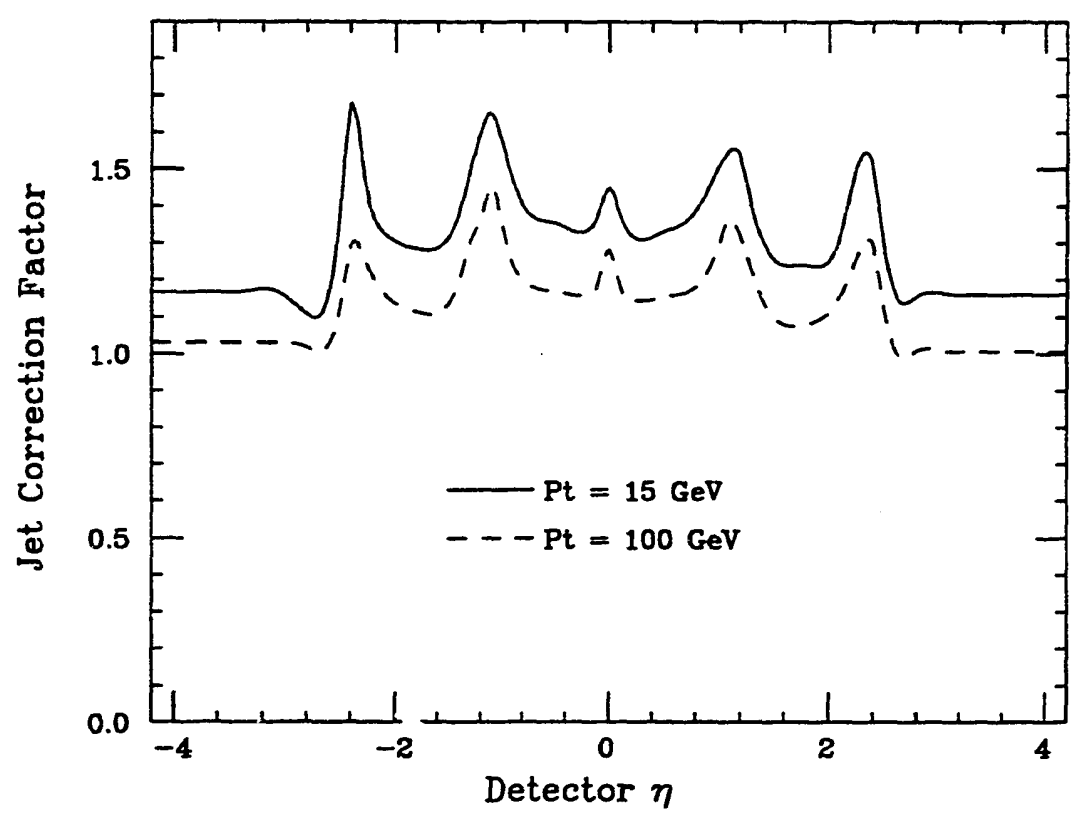

Figure 5.6: The multiplicative correction factor applied to the measured jet $P_{t}$, shown as a function of $\eta$. The solid line is for jets which have an uncorrected $P_{t}$ of $15 \mathrm{GeV}$. The dashed line shows the corrections for jets which have an uncorrected $\mathrm{P}_{t}$ of $100 \mathrm{GeV}$. 
transverse energy (because it sums the transverse energy in each tower). The absolute value of the vector $\overrightarrow{\mathrm{H}}_{t}$ will be referred to as $\mathbb{Z}_{t}$.

\subsubsection{Missing $\mathrm{E}_{t}$ Corrections}

The $\mathbb{Z}_{t}$ is a sum over all calorimeter towers; therefore, this sum will include the $W$ electron, any jets in the event, and the remaining particles (the underlying event). Any corrections applied to these quantities should also be reflected in corrections to $\overrightarrow{\mathrm{E}}_{t}$.

The procedure described in this section for correcting the $\mathbb{Z}_{t}$ in $\mathrm{W}$ events was developed in [16] for CDF's measurement of the transverse momentum distribution of the $\mathrm{W}$ boson.

\subsubsection{Electron energy corrections to $\overrightarrow{\mathrm{H}}_{t}$}

The electron corrections are applied to the $\mathbb{Z}_{t}$ by first removing the electron's contribution to the $\mathrm{Z}_{i}$. This is done by adding each tower in the electron clusterover the $\mathbb{Z}_{t}$ tower threshold-to $\overrightarrow{\mathrm{H}}_{t}$, that is:

$$
\overrightarrow{\mathrm{E}}_{t} \rightarrow \overrightarrow{\mathrm{E}}_{t}+\sum_{i} E_{i} \sin \theta_{i} \hat{n}_{i}
$$

where $i$ runs over all electron towers above the threshold. Then the corrected electron momentum is subtracted from $\overrightarrow{\mathrm{H}}_{t}$ :

$$
\overrightarrow{\mathrm{E}}_{t} \rightarrow \overrightarrow{\mathrm{H}}_{t}-\vec{P}_{\text {electron }}
$$

\subsubsection{Jet energy corrections to $Z_{t}$}

The jet cluster corrections are applied in the same manner as the electron corrections-first remove uncorrected towers and then add the fully corrected jet vector. The tower thresholds used in the jet clustering algorithm are identical to the thresnolds used in the $\mathbb{Z}_{t}$ summation; therefore, no double counting of towers will occur. The underlying event correction was not applied to the jets when correcting the $\mathrm{F}_{t}$ (again, this is done to insure that no energy is unaccounted for or double counted). (Only clusters with uncorrected $E_{t}$ greater than $10 \mathrm{GeV}$ were used; clusters with le.ss $\mathrm{E}_{t}$ are grouped in with the unclustered energy correction. 


\subsubsection{Muon corrections to $\mathbb{Z}_{t}$}

Muon corrections were only made for the muon from the W decay in W $\rightarrow \mu \nu$ events; no corrections were made for the $\mathrm{W} \rightarrow e \nu$ events ${ }^{1}$.

To correct for the muon the $E_{t}$ in the calorimeter tower traversed by the muon is removed from the $\overrightarrow{\mathrm{H}}_{t}$ sum, then the momentum of the muon (as measured by the track) is subtracted from $\overrightarrow{\mathrm{\psi}}_{t}$.

\subsubsection{Unclustered-energy corrections to $\mathbb{Z}_{t}$}

Any energy in the calorimeters not included in the above categories is termed the "unclustered energy". This energy will tend to be undermeasured because of detector cracks and calorimeter nonlinearities. The correction factor for the unclustered energy was determined using Monte Carlo $W$ events and studying the relationship between the measured vector for the unclustered energy and that obtained from the known particles that were generated by the Monte Carlo. On average the net observed transverse momentum of the underlying event was $50 \%$ of the generated value. To correct the underlying event, its net transverse momentum is multiplied by 2 . The components of the unclustered energy vector both parallel and perpendicular to the $\mathrm{W}$ direction were studied and the correction factor was consistent with 2.0 in both directions[16].

\section{5 $\mathbf{W}+$ Jet $\rightarrow \mathrm{e}+\nu+$ Jet Event Selection}

At this point the data sample consists of events accepted by the electron trigger online, and then required to have a $20 \mathrm{GeV} \mathrm{P} \mathrm{P}_{t}$ electron candidate offline. In order to get a sample of $\mathrm{W}+$ jet events in which the $\mathrm{W}$ decayed into an electron and a neutrino, the following cuts are applied to the events:

1. High $\mathrm{P}_{\ell}$ electrons can come from many sources in proton-antiproton collisions. W events, however, contain a neutrino which usually has high $P_{t}$ as well. The corrected missing $E_{t}\left(Z_{t}\right)$ is required to be greater than $20 \mathrm{GeV}$. The distribution of $\mathrm{Z}_{t}$ is shown in figure 5.7 ; this plot actually shows the $\mathbb{Z}_{t}$ after all other cuts in this list have been applied except for the transverse-mass cut. The events at low $耳_{t}$ are coming from $\mathrm{Z}$ bosons, QCD dijets, photon conversions, and heavy-quark production.

2. The events are required to have at least one jet in them with a corrected $\mathrm{P}_{t}$ greater than $15 \mathrm{GeV}$ (the electron will produce a jet cluster; therefore, care is taken to remove this cluster before making the jet requirement).

\footnotetext{
${ }^{1}$ High $\mathrm{P}_{t}$ muons are not expected in $\mathrm{W} \rightarrow e \nu$ events; the effect of any muons resulting from jets or the underlying event would be included in their respective corrections
} 

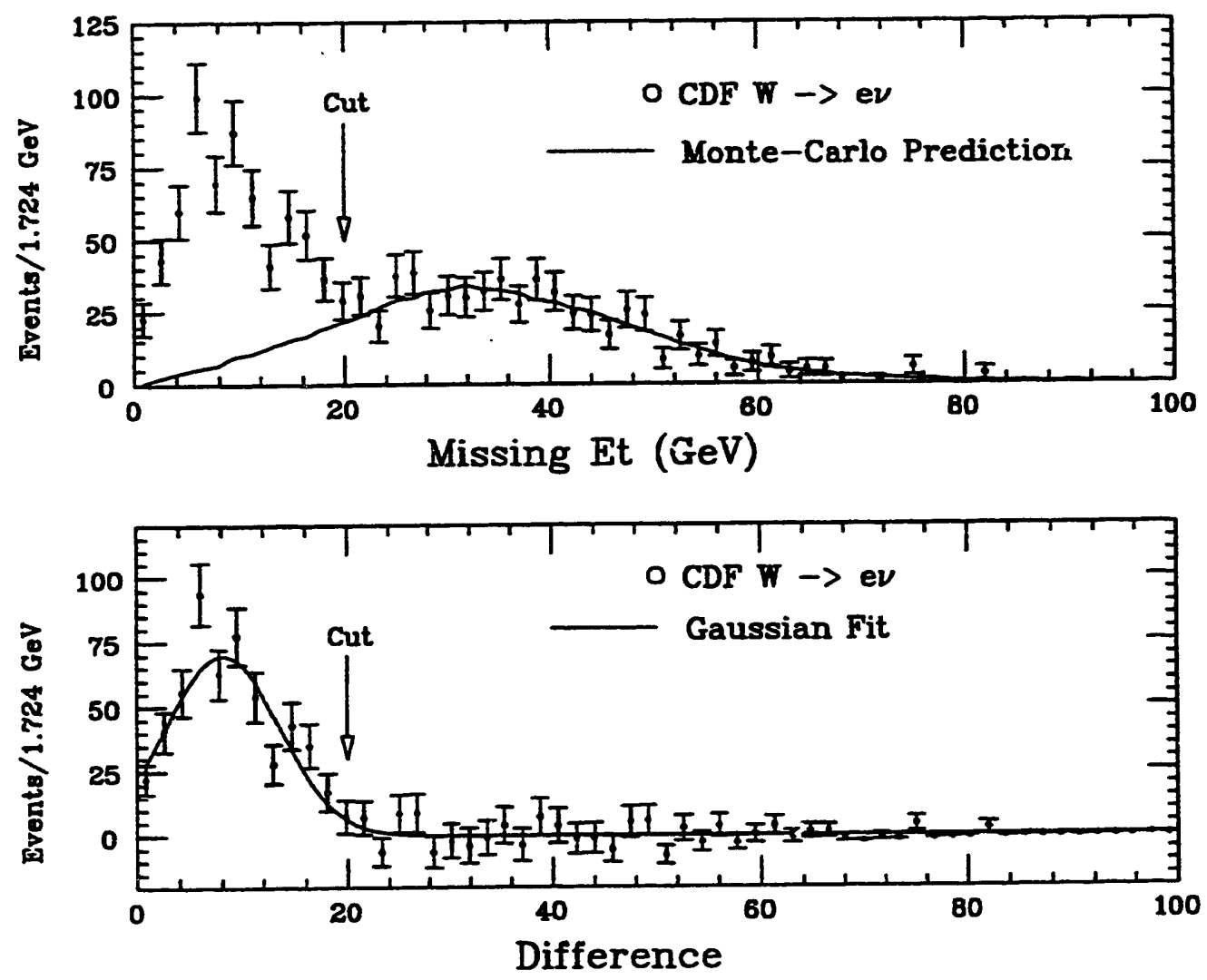

Figure 5.7: The upper plot shows the missing- $E_{t}$ for the $W+$ jet candidate events with the $20 \mathrm{GeV}$ cut removed. A prediction, using VECBOS and detector simulation, is shown for comparison. The Monte Carlo is normalized to the data above $30 \mathrm{GeV}$. The lower plot shows the result of subtracting the Monte Carlo prediction from the data. The solid curve is a gaussian fit (notice that the $y$-axis of the plot extends below zero). 
3. The electron and the jet are required to be separated by more than 0.9 in $\eta-\phi$ space (i.e. $\sqrt{\left(\eta_{\text {Jet }}-\eta_{e}\right)^{2}+\left(\phi_{\text {Jet }}-\phi_{e}\right)^{2}}>0.9$ ). This cut removes only $1.2 \%$ of the data, because the energy isolation cut, already applied to the electron, implicitly removes events in which the jet is close to the electron.

4. A possible background is $\mathrm{Z}$ boson + jet events in which the $\mathrm{Z}$ decays to $e^{+} e^{-}$. If one of the electrons is measured with too much or too little energy it could fake the missing $E_{t}$ signature of the neutrino. To reduce this background a cut is made to remove events in which a second electron candidate-defined with much less stringent identification cuts-forms an invariant mass, with the main electron candidate, that is between 70 and $110 \mathrm{GeV}$ ( $20 \mathrm{GeV}$ around the $\mathrm{Z}$ boson mass). The loose cuts on the second electron candidate are:

- All second electron candidates (regardless of which calorimeter they enter) are required to meet the following

- Ratio of hadronic to electromagnetic energy $\mathrm{H} / \mathrm{E}<0.1$

- Isolation (defined in section 5.1.2) $<0.1$

- Cluster is in the fiducial region of the calorimeter

- Transverse energy of the cluster is greater than $0 \mathrm{GeV}$

- If the electron cluster is in the central calorimeter

- Cluster is required to have an associated track with $\frac{E}{P}<2$

- Charge of the track should be the opposite sign of the main electron's charge.

- If the electron cluster is in the plug calorimeter

- If the cluster has an associated track (the tracking efficiency drops off quickly in the region $1<\eta<2$ ) then it should be opposite in sign to the main electron candidate.

- There should be hits in the VTPC that are consistent with a track pointing at the electron cluster (the VTPC is efficient in the plug region but cannot determine momentum, nor measure the $\phi$ coordinate of the track).

- If the electron cluster is in the forward calorimeter, no further cuts are applied.

5. Another background expected to be in the sample is photon + jet events in which the photon converts in the detector material (e.g. the beam pipe or the VTPC) into two electrons. This background is reduced by making two cuts. First, the electron track is projected backwards into the VTPC. 
It is then required that there be at least $20 \%$ of the possible VTPC hits be found for this track This cut rejects photons that convert on the outside of the VTPC(there is a relatively large amount of material at the outside of the VTPC). Second, the event is searched for any tracks that have a charge opposite to that of the main electron's, which are within $5^{\circ}$, in $\theta$, of the electron track, and which have a distance of closest approach to the electron track less than $2 \mathrm{~cm}$. Any tracks passing these cuts are assumed to be the other electron from the photon conversion, so the main electron would be rejected.

6. If the electron and neutrino come from the decay of a $\mathrm{W}$ boson, then the invariant mass of the two combined should be large. The "transverse mass" $\left(\mathrm{M}_{t}\right)$ of the electron and neutrino system is defined to be the invariant mass that results when the z-components of the electron and neutrino are both taken as zero. Figure 5.8 shows the $\mathrm{M}_{t}$ distribution for the data compared to a Monte Carlo prediction; the figure shows an excess of events at small values of $M_{t}$. The transverse mass is required tc be greater than $20 \mathrm{GeV}$.

The $\mathrm{W}(\rightarrow e \nu)+$ Jet candidates sample, as described so far, consists of 411 events. The backgrounds remaining in sample are discussed in detail in Chapter 8; the total background contamination is estimated there to be $7.4 \%$.

\subsection{W + Jet $\rightarrow \mu+\nu+$ Jet Event Selection}

Again, the muonic decay mode of the $\mathrm{W}$ is not used in the $\cos \theta_{s}^{*}$ measurement, but is used in Chapter 11 to search for excited quark states decaying into a W and a jet. The selection of muonic $\mathrm{W}+$ Jet events is very similar to the selection of the electron events. The requirements are:

\section{Corrected $\mathrm{B}_{t}>20 \mathrm{GeV}$}

2. At least one jet with corrected $\mathrm{P}_{t}>15 \mathrm{GeV}$

3. The muon and the jet are required to be separated by more than 0.25 in $\eta-\phi$ space. This cut is smaller than the cut applied to electrons, because the muon isolation cuts accept muons closer to jets. These lepton-jet separation cuts are applied, not to reduce backgrounds in the data, but to make the treatment of Monte Carlo and data events more uniform (the Monte Carlo dues not simulate the necessary information to make the lepton isolation cuts). 


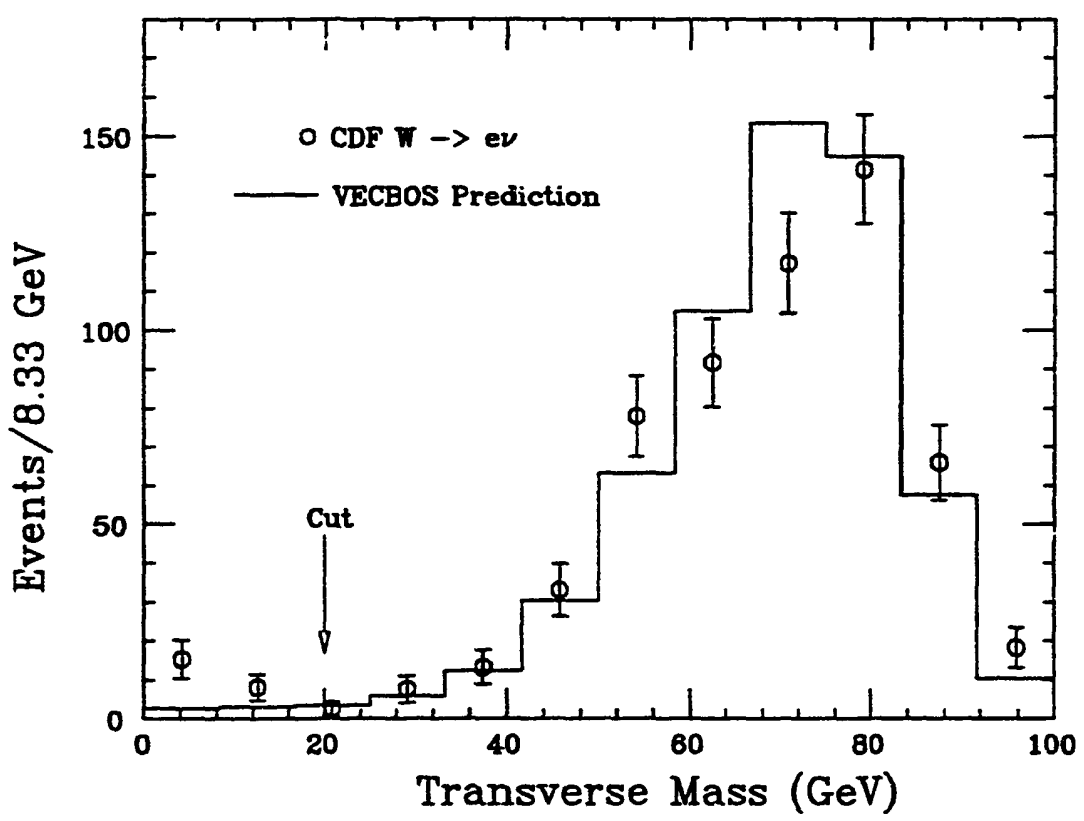

Figure 5.8: The transverse-mass distribution for the $W+$ jet candidate events. The solid line is a Monte Carlo prediction using vесвos and a detector simulation. The $20 \mathrm{GeV}$ cut was removed in order to make this plot; a small background contamination can be seen below the cut. 

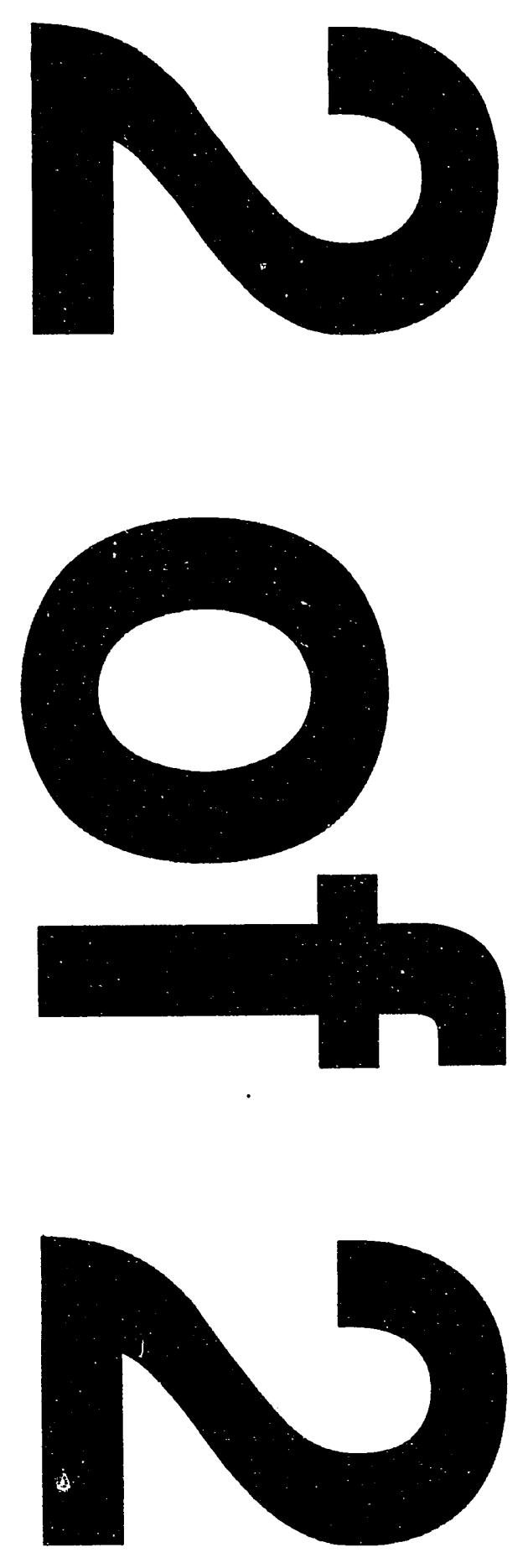
4. $\mathrm{Z}$ boson background is reduced by requiring that there be no other muon candidate in the event, passing less restrictive identification cuts, and having $\mathrm{P}_{t}>20 \mathrm{GeV}$. The second muon candidate is required to meet all normal muon identification cuts except:

- The $r \phi$ matching of the muon stub with the CTC track is required to be within $10.0 \mathrm{~cm}$ (the normal cut is $2.0 \mathrm{~cm}$ )

- No isolation cut is used

The second muon candidate can also be what is known as a "central minimum ionizing object". These objects are selected in the same way as the normal muon candidates except that no signal is required in the central muon detector (i.e. essentially a high $P_{t}$ track pointing to a tower in the calorimeter with very little energy in it).

5. Cosmic ray muons are removed by looking at the timing information from the central hadronic calorimeter, and requiring the muon's energy deposition to be within a time window consistent with the time of the protonantiproton collision (as measured by the beam-beam counters).

6. The transverse mass is required to be greater than $20 \mathrm{GeV}$.

The $\mathrm{W}(\rightarrow \mu \nu)+$ Jet candidates sample, as described so far, consisis of 278 events. The backgrounds in this sample have not been directly estimated, because the search for heavy particles does not require this information. The backgrounds in the inclusive muonic $W$ events (i.e. no specific jet requirements) have been estimated[18] and are small (a few percent). The number of events in the muonic $\mathrm{W}+$ jet sample is about $60 \%$ of the number of events in the electronic $\mathrm{W}+$ jet sample; this indicates that the sample is relatively free of backgrounds (because the tighter $\eta$ cut on the muon should make the ratio roughly $60 \%$ ). 


\section{Chapter 6}

\section{Simulation Tools and Comparison with Data}

The $\mathrm{W}+$ jet candidate sample has now been defined. Before the further analysis of these event is discussed, it is necessary to describe the Monte Carlo programs that will be used to simulate these events. Being able to simulate the $\mathrm{W}+$ jet events is necessary in order to understand the effects of the CDF detector on the $\cos \theta_{s}^{*}$ distribution. Event simulation is also necessary in order to make predictions about the expected backgrounds in the $W+$ jet candidate sample.

This chapter describes each of the simulation programs used for the analysis, and concludes by showing comparisons between the CDF data and the Monte Carlo predictions (which is also appropriate now in order to demonstrate that the $\mathrm{W}+$ jet sample is well understood).

The Monte Carlo generators used for this analysis simulate proton-antiproton collisions at $1.8 \mathrm{TeV}$. The output of the generators is a list of the particles produced in the interaction along with the 4-momentum of each particle. This particle list can then be used on its own for studying the properties of the events, or it can be fed into a detector simulation program. The detector simulation programs take the list of particles from the generator, simulate the response of the detector to these particles, and produce an output in the same format as the real CDF data.

\subsection{Parton Level Simulation}

This section describes the VECBOS event generator and the QDJTMC detector simulation. These two programs were used for all Monte Carlo studies done in this analysis, except for the estimates of the background contamination. 


\subsubsection{VECBOS Event Generator}

The VECBOS generator[7], already discussed in Chapter 3, is a parton level generator. This means that the list of outgoing particles that it produces contains quarks and gluons. Having this simple output format makes it easy to study the properties of the events before detector simulation. For example, in order to study the effects of the detector, the $\cos \theta_{s}^{*}$ distribution before and after the detector simulation can be compared. This is one of the reasons that a parton level generator was chosen.

The output from the VECBOS generator was used in Chapter 3 to illustrate the analysis method used.

VECBOS is a leading order Monte Carlo (see section 2.3), which generates events according to matrix elements of the type shown in figure 2.3. The parton distribution functions (described in section 2.1) used are the HMRSB[19] set. The momentum scale (see section 2.3.1) for evaluating the pdf and the strong coupling constant, $\alpha_{s}$, was chosen to be $1 / 2$ of the $\mathrm{P}_{t}$ of the jet (or equivalently: of the W).

Another parton level generator, called PAPAGENo[20], will be used in section 8.2 to estimate the QCD dijat contamination in the event sample. PAPAGENO is a parton level generator just like VECBOS, except that PAPAGENO can simulate many kinds of physics (VECBOS only simulates $\mathrm{W}$ and $\mathrm{Z}$ boson production).

\subsubsection{QDJTMC Detector Simulation}

VECBOS outputs the momentum of the electron and neutrino from the $\mathrm{W}$ boson decay as well as the momentum of the quark (or gluon). The CDF collaboration has developed a program, called QDJTMC, that takes the output of generators like VECBOS and simulates the effect of the CDF detector. The following sections describe how QDJTMC simulates the events produced by VECBOS.

\section{Intrinsic $\mathrm{K}_{t}$}

The first step in the QDJTMC simulation is to read in the information output from VECBOS, and apply a small transverse boost to the event. This boost simulates the "intrinsic $\mathrm{K}_{t}$ " discussed in section 2.3.4. Intrinsic $\mathrm{K}_{t}$ is a result of the emission of many low- $P_{t}$ gluons and quarks in the interaction. QDJTMC gives the entire $W+$ jet system a transverse momentum chosen from a double-gaussian distribution$75 \%$ of the events have their $\mathrm{K}_{t}$ chosen from a gaussian of width $9.5 \mathrm{GeV}$, and $25 \%$ of them from a gaussian of width $4.0 \mathrm{GeV}$. The transverse momentum was applied in a random $\phi$ direction.

These numbers for the applied $K_{t}$ come from a fit to the measured transverse momentum in CDF dijet data. The momentum of the $\mathrm{W}+$ jet system in the CDF 
data will be compared to the VECBOS-QDJTMC prediction in section 6.3 .

\section{Electrons}

QDJTMC simulates electrons by smearing their transverse momentum with a gaussian resolution:

$$
\sigma_{\mathrm{P}_{t}}=\sqrt{\left(0.135 \sqrt{\mathrm{P}_{t}}\right)^{2}+\left(0.02 \mathrm{P}_{t}\right)^{2}}
$$

The electron is also projected through the CDF detector to where it would hit the central electron strips (this position is used to define the fiducial region of the detector).

These two properties of the electron are all that are needed by the analysis. The analysis includes a cut separating the electron from the jet, so that QDJTMC does not have to simulate the effect of the jet Jverlapping the electron.

\section{Jets}

QDJTMC simulates jets by applying the inverse of the jet corrections described in section 5.3.1. The jet is also smeared using a gaussian resolution as measured from the data (the smearing used is approximately $\sigma_{\mathrm{P}_{t}}=\mathrm{P}_{t}$, but varies depending on what part of the detector the jet goes into). The $\eta$ and $\phi$ of the jet are also smeared with gaussian resolutions, as determined from Monte Carlo studies which used more detailed jet fragmentation simulation (the $\eta$ and $\phi$ resolutions are both approximately 0.1 , but vary significantly with $\mathrm{P}_{t}$-becoming smaller at higher $\left.\mathrm{P}_{t}\right)$.

Many comparisons between the QDJTMC jet simulation and CDF multijet data[21, 22] have been made, and the comparisons are very good.

\section{Missing $\mathrm{E}_{t}$}

The missing- $\mathrm{E}_{t}$ vector $\left(\overrightarrow{\mathrm{E}}_{t}\right)$ was defined in section 5.4 ; it is used to estimate the momentum of the neutrino. Several steps are involved in the QDJTMC simulation of $\overrightarrow{\mathrm{H}}_{t}$ :

1. $\overrightarrow{\mathbb{E}}_{t}$ is initialized to zero.

2. The jet momentum, after simulation, is subtracted from $\overrightarrow{\mathrm{H}}_{t}$

3. The electron, after simulation, is subtracted from $\overrightarrow{\mathrm{H}}_{t}$

4. The intrinsic $K_{t}$ boost applied to the event was assumed to be caused by recoiling low-energy particles. These recoiling particles have total momentum $\mathrm{R}_{t}=-\mathrm{K}_{t}$, and should deposit some energy in the calorimeter. 
To simulate this energy deposition, $R_{t}$ is first smeared, to account for detector resolution, with a gaussian of width $\sigma_{R_{t}}=0.85 \sqrt{R_{t}}$ (this resolution was determined using Monte Carlo detector simulations). The resulting vector is then divided by 2.0 , to account for non-linear detector response and detector cracks. This vector is then subtracted from $\overrightarrow{\mathrm{H}}_{t}$. This method for simulating the $K_{t}$ was used in CDF's measurement of the mass of the $\mathrm{W}$ boson[18]. Comparisons of the $\mathrm{K}_{t}$ for the data and the Monte Carlo will be shown in section 6.3 .

5. The underlying event was described in section 2.1 ; it is the particles originating from the partons that did not participate in the hard scattering. The underlying event is simulated by first generating a total scalar $E_{t}$, $\sum E_{t}$, which is the scalar sum of the transverse energy in all calorimeter towers. The $\sum E_{t}$ was generated using a gaussian distribution with average $27 \mathrm{GeV}$ and width $18 \mathrm{GeV}$ (these numbers are a fit to the data, shown in figure 6.1). This $\sum E_{t}$ is assumed to be distributed uniformly in $\phi$, and so its contribution to $\overrightarrow{\mathrm{E}}_{t}$ will be due to its fluctuations. This is simulated by generating an underlying event vector, in a random $\phi$ direction, with magnitude chosen using a gaussian distribution with average zero and width $\sigma=0.67 \sqrt{\sum \mathrm{E}_{t}}$ (this resolution was determined using Monte Carlo detector simulations). This vector is divided by 2.0 to account for nonlinearities and cracks, and then added to $\overrightarrow{\mathrm{H}}_{t}$. This method for simulating the underlying event contribution to $\overrightarrow{\mathrm{H}}_{t}$ was also used in the CDF W mass measurement[18].

\section{Event Vertex}

QDJTMC also simulates the distribution of the $z$ position of the proton-antiproton collisions. This is done by generating a gaussian with average $4.5 \mathrm{~cm}$ and width $35 \mathrm{~cm}$.

\section{Muons}

Although muons are not used for the $\cos \theta_{s}^{*}$ measurement, they will be used in the excited quark search in Chapter 11. QDJTMC simulates muons by smearing their momentum according to the tracking resolution, $\sigma_{\mu}=0.0011 \mathrm{P}_{t}{ }^{2}$. The muon is also projected through the CDF detector to determine where it hit the central muon chambers (in order to make fiducial cuts on the muon). Note that nothing needs to be done with muons in the $\overrightarrow{\mathrm{H}}_{t}$ calculation, because they don't deposit energy in the calorimeters (actually muons can deposit a small amount of energy, but this level of detail is unnecessary for this analysis). 
There are many advantages to using the parton level simulation just described, not the least of which is that the QDJTMC simulation requires very little CPU time in order to simulate the detector ( therefore, large numbers of event can be generated).

\subsection{Hadron Level Simulation}

In order to estimate the backgrounds contaminating the $\mathrm{W}+$ jet sample (which is described in Chapter 8), a Monte Carlo package capable of generating the events and simulating the detector in much more detail is necessary. This section describes the Monte Carlo programs that were used to estimate the backgrounds in the $\mathrm{W}+$ jet sample. The generator is called Pythia, and the detector simulation is called QFL.

\subsubsection{Pythia Event Generator}

The output from the Pythia[23] generator does not contain quarks or gluons, instead the program produces a list of real particles that exit the interaction region (i.e. mesons and baryons). Pythia uses a "string fragmentation" model to transform quarks and gluons into hadrons, this was mentioned in section 2.3.3. Pythia also has a model for simulating the intrinsic $K_{t}$ of the hard scattering system (by actually radiating many low energy quarks and gluons). Pythia also has a model for the underlying event. These models used by Pythia are not much more than "hand waving" arguments based on our limited understanding of lowenergy QCD; however, it has been extensively tuned to agree well with data from many experiments (including $e^{+} e^{-}$collisions).

Another Monte Carlo generator, called HERWIG[24], will also be used in calculating the backgrounds. HERWIG is very similar to Pythia; however, it uses different models for the parton fragmentation and the underlying event (which include quantum interference effects between low $\mathrm{P}_{t}$ gluons). HERWIG was used to estimate the top quark background that might be present in the data sample (which is treated as a systematic uncertainty in the $\cos \theta_{s}^{*}$ measurement).

\subsubsection{QFL Detector Simulation}

The Pythia events are run through the QFL detector simulation. QFL simulates each particle from Pythia's output list by propagating it through the detector and depositing its energy appropriately in the detector. For electrons, an electromagnetic shower model is used to simulate the energy deposition in the calorimeters and strip chambers. For hadrons, an hadronic shower model is used to simulate 
the energy deposition in the hadronic calorimeters. The simulation models used by QFL have several parameters which have been carefully tuned to match the CDF testbeam data, and have been shown to do a good job of simulating the detector's response to leptons, photons, and hadrons.

The output from QFL is in a form that can be input to the electron and jet clustering algorithms (discussed in sections 5.1.1 and 5.3), as well as the algorithm that calculates the missing- $\mathrm{E}_{t}$ (section 5.4).

The detailed simulation of the detector response provided by QFL, and the ability to use the clustering algorithms, is necessary for estimating the background contamination in the data sample.

QFL takes much more CPU time per event than does QDJTMC which is one reason it was not used instead of VECBOS-QDJTMC (two million VECBOS events were generated and run through QDJTMC).

\subsection{General Comparison with CDF Data}

Figures 6.2 to 6.11 show comparisons, for various kinematic distributions, of the VECBOS-QDJTMC simulation with the CDF data. The Monte Carlo predictions were normalized to have the same number of events as the data. The rest of this section discusses these figures in more detail.

Figures 6.2, 6.3,6.4, and 6.5 show the transverse momentum distribution for the electron, the neutrino, the jet, and the W. The predictions of the VECBOSQDJTMC combination agree very well with the data.

Figure 6.6 shows the $\eta$ distribution of the electron. As was discussed in section 3.1.2, the $\mathrm{W}^{+}$events have been inverted in making this plot. As a result, the Monte Carlo prediction is slightly asymmetric (the distribution would be symmetric if the $\mathrm{W}^{+}$events were not inverted). The data seems to be slightly lower than the Monte Carlo near $\eta=1$. The difference is small enough to be a statistical fluctuation. The Pythia-QFL Monte Carlo predicts the same $\eta$ spectrum as VECBOS-QDJTMC does. In any case, the $\cos \theta_{s}^{*}$ distribution is very insensitive to any $\eta$ dependent detector effects that might be causing this (as will be discussed in later chapters).

Figure 6.7 shows the $\eta$ distribution for the leading jet in the event. The Monte Carlo agrees well with the data. This distribution is also inverted for $\mathrm{W}^{+}$events, and is slightly asymmetric (this asymmetry is due to the fact that up-quarks have a harder momentum spectrum than do down-quarks, so the $\mathrm{W}+$ jet system tends to be boosted more along the up-quark direction).

Figure 6.8 shows the $\mathrm{P}_{t}$ distribution of the underlying event. The underlying event is defined similarly to the "unclustered energy" defined in section 5.4.1.4, except that if the leading jet had an uncorrected $P_{t}$ of less than $10 \mathrm{GeV}$ it was not 


\begin{tabular}{||c|c|c||}
\hline Number of Jets & Number of Events & Number of Events in Final Sample \\
\hline 1 & 324 & 117 \\
2 & 70 & 37 \\
3 & 14 & 8 \\
4 & 3 & 3 \\
\hline
\end{tabular}

Table 6.1: The number of events containing $n$ jets with corrected $P_{t}>15 \mathrm{GeV}$ (the W + jet sample was defined to have at least one jet with corrected $P_{t}>15$ ). The second column shows the number of events in the $W+$ jet sample described so far. The third column shows the number of events in the final sample used for the $\cos \theta_{s}^{*}$ measurement.

included in the underlying event (it was included in the unclustered energy). The agreement with the data is very good, which is not entirely unexpected since this is the result of the artificial $\mathrm{K}_{t}$ kick introduced by QDJTMC-which was tuned to match other CDF data samples.

Figure 6.9 shows the transverse momentum distribution of the $\mathrm{W}+$ jet system. This quantity should be the same as the underlying event, unless there is more than one jet present in the event. The figure shows that the data has a. harder spectrum than the Monte Carlo predicts. This is because VECBOS only generates one-jet events, and the data does contain multi-jet events (Table 6.1 shows the distribution of the number of jets in each event). The $\cos \theta_{s}^{*}$ measurement is very insensitive to the $P_{t}$ of the $W+$ jet system, because $\cos \theta_{s}^{*}$ is defined in the center-of-mass of the system.

Figure 6.10 shows the transverse-mass distribution. This distribution is sensitive to the details of the missing- $\mathrm{E}_{t}$ simulation. The plot shows good agreement between the data and the Monte Carlo.

Figure 6.11 shows the distribution of the $\mathrm{z}$-component of the neutrino, $\mathrm{P}_{z}^{\nu}$. This quantity can only be obtained after constraining the neutrino-electron system to the $\mathrm{W}$ mass (as discussed in section 3.2.1 and section 7.1). Since $\mathrm{P}_{z}^{\nu}$ is dual-valued, the selected solution is plotted. This distribution is also inverted for $\mathrm{W}^{+}$events. The distribution is very asymmetric which is mostly an artifact of the definition of the selected solution (the method used to choose the selected solution tends to choose the more negative solution for $\mathrm{P}_{z}^{\nu}$ ).

The figures shown in this chapter demonstrate that the Monte Carlo is doing an excellent job of representing the data. Therefore, we can be confident in using the Monte Carlo prediction to study the effect of the detector on the $\cos \theta_{s}^{*}$ distribution. The Monte Carlo will also be used, in Chapter 9, to study the effects, on $\cos \theta_{s}^{*}$, of systematic uncertainties. 


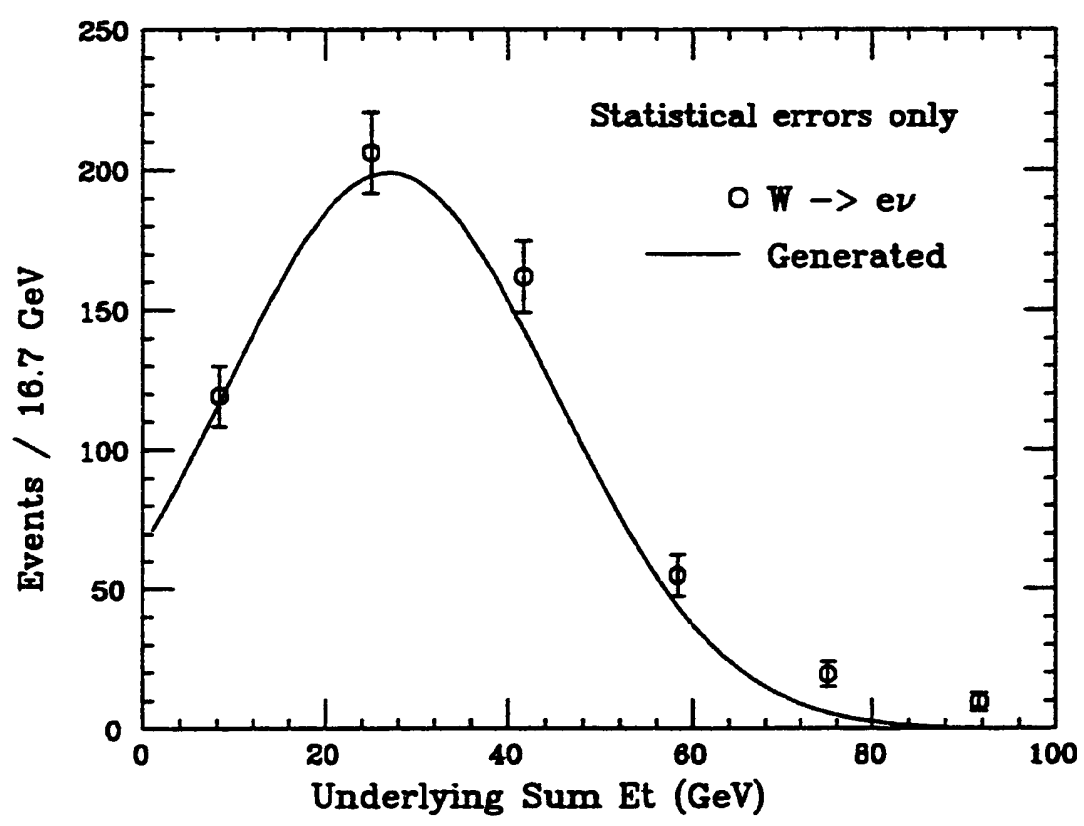

Figure 6.1: Underlying Event $\sum E_{t}$, in the W + Jet sample, compared to the VECBOSQDJTMC generation. The underlying $\sum \mathrm{E}_{t}$ is the total scalar $\mathrm{E}_{t}$ in the detector after removing all contributions attributed to the hard scattering. The curve shows the fit used to generate the events, not the output of the Monte Carlo (not enough information was stored to reconstruct this quantity from the Monte Carlo events). 


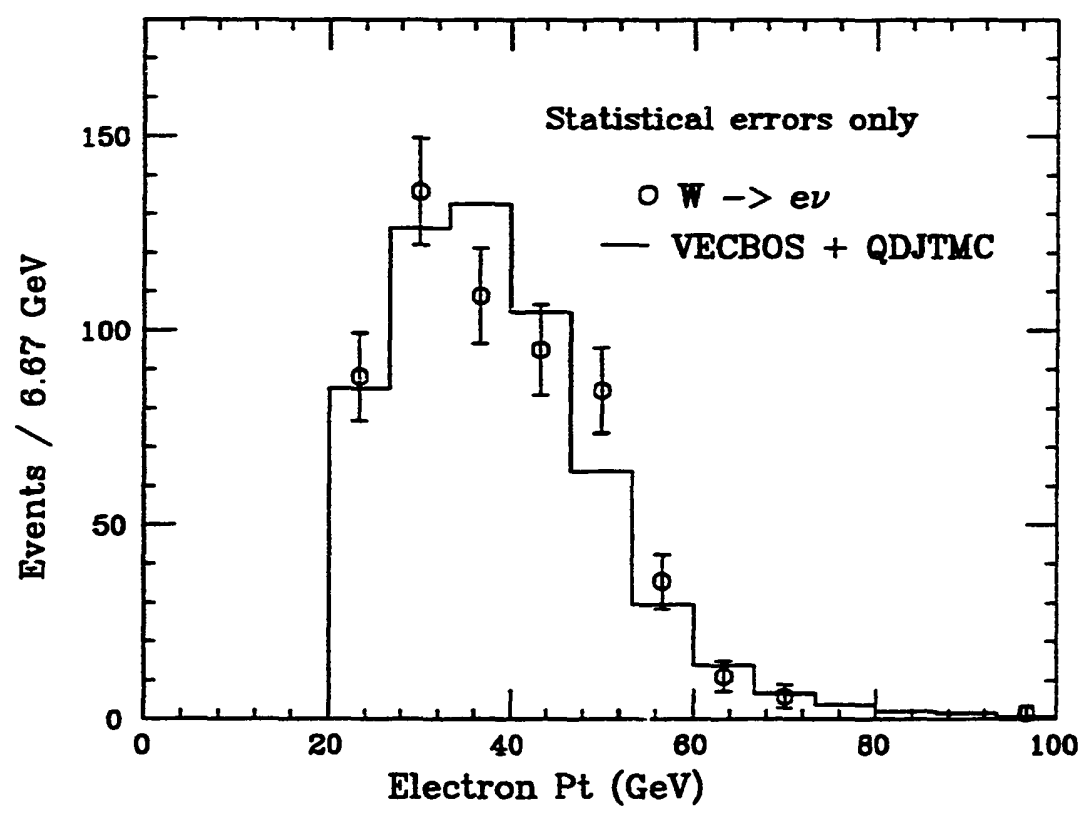

Figure 6.2: Electron transverse momentum, in the $W+$ Jet sample, compared to the VECBOS-QDJTMC prediction. This quantity was cut at $20 \mathrm{GeV}$.

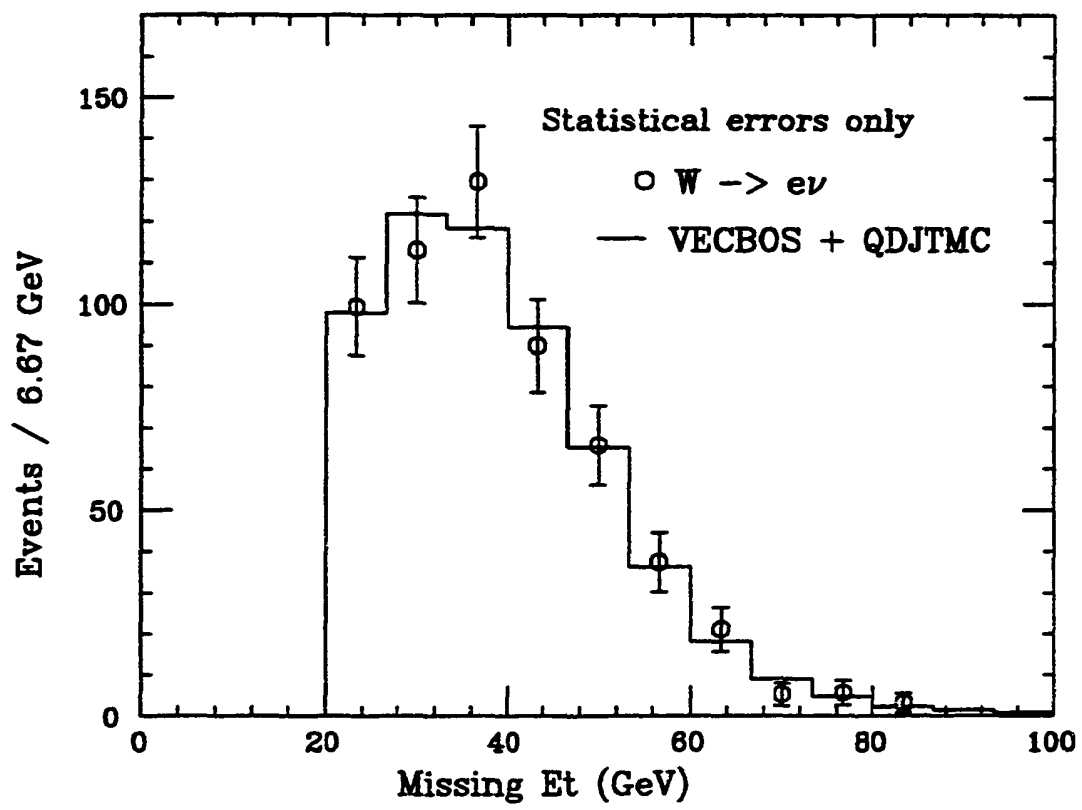

Figure 6.3: Missing- $\mathrm{E}_{t}$, in the $\mathrm{W}+$ Jet sample, compared to the VECBOS-QDJTMC prediction. This quantity was cut at $20 \mathrm{GeV}$. 


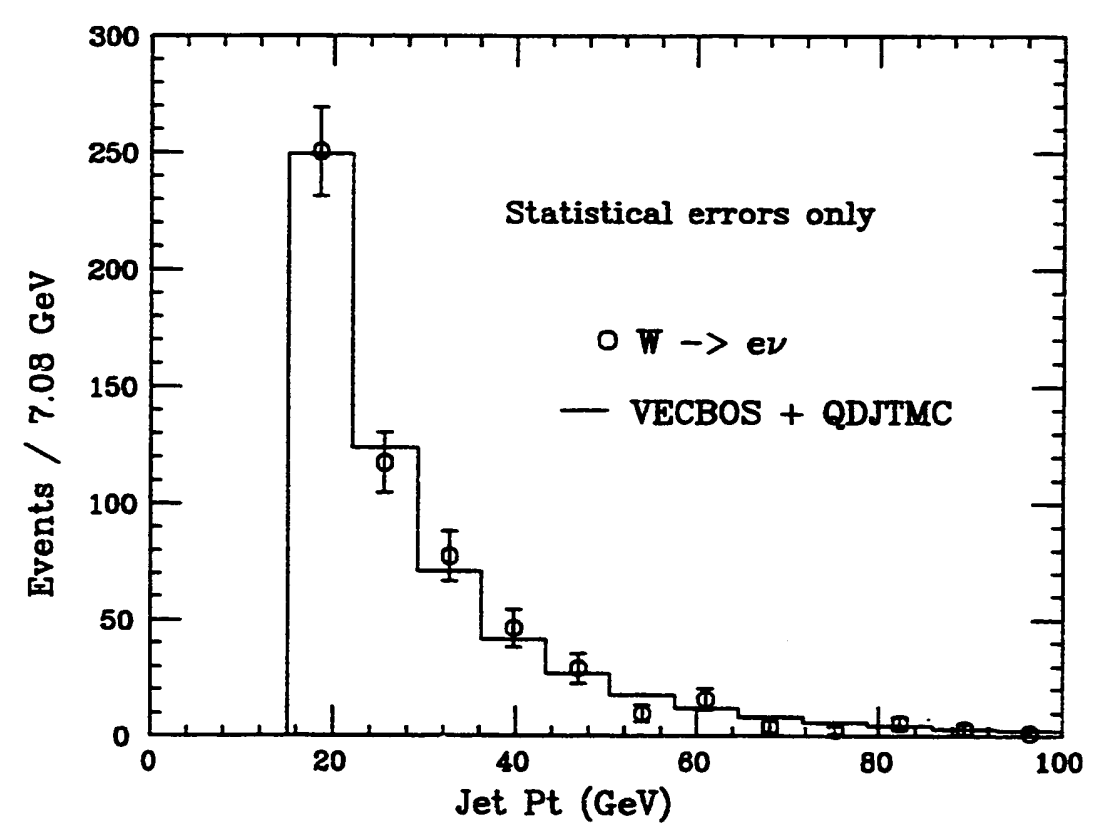

Figure 6.4: Leading-jet transverse momentum, in the $W+$ Jet sample, compared to the VECBOS-QDJTMC prediction. This quantity was cut at $15 \mathrm{GeV}$.

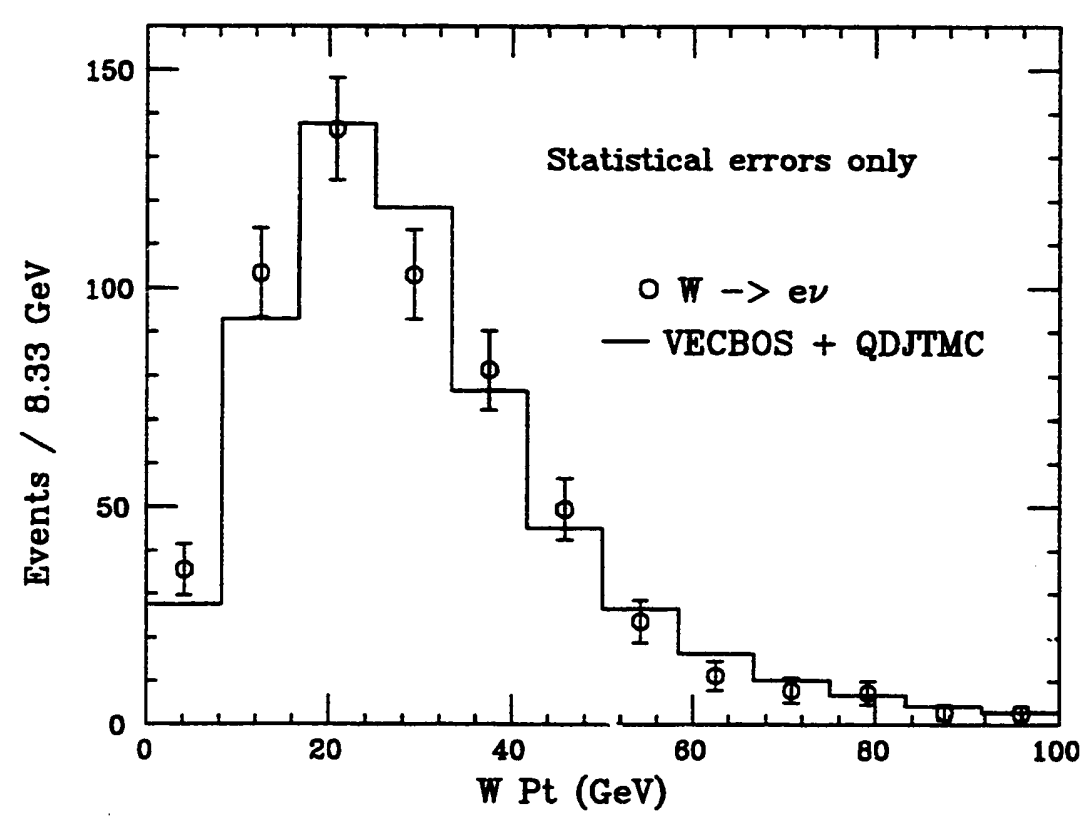

Figure 6.5: $W$ transverse momentum, in the $W+$ Jet sample, compared to the VECBOS-QDJTMC prediction. 


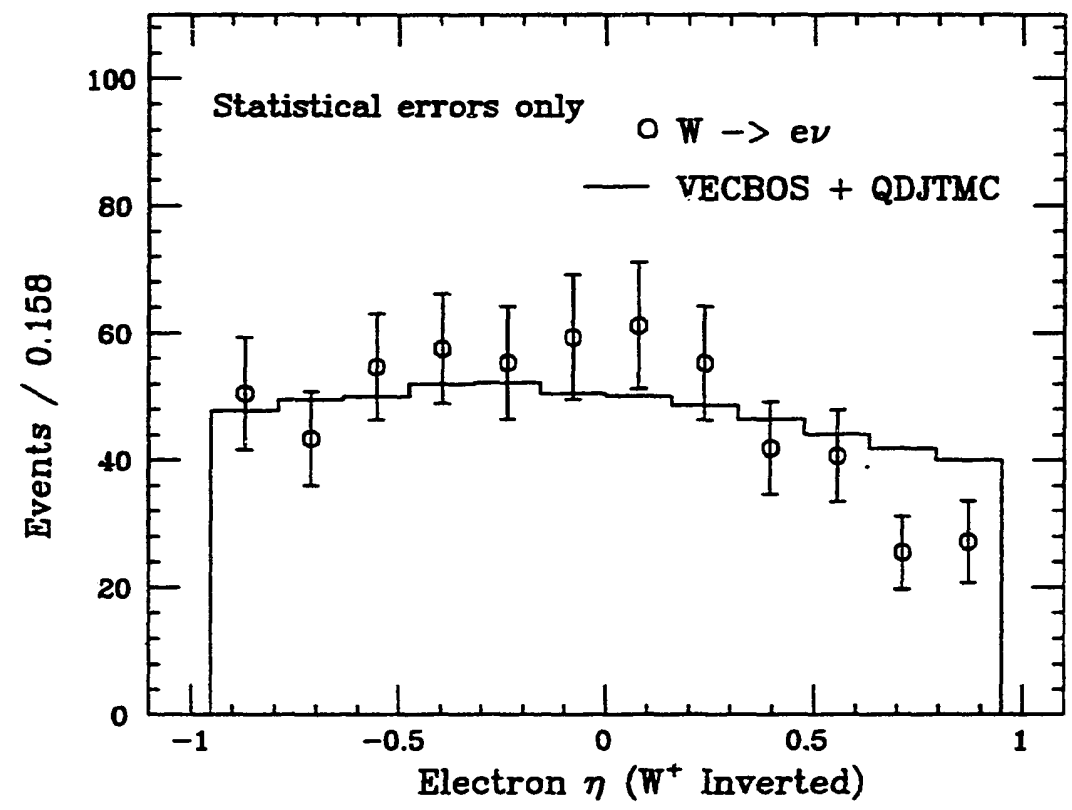

Figure 6.6: Electron pseudo-rapidity, in the $W+$ Jet sample, compared to the VECBOS-QDJTMC prediction. $\mathrm{W}^{+}$events are inverted in this distribution. This quantity was cut at \pm 0.95 .

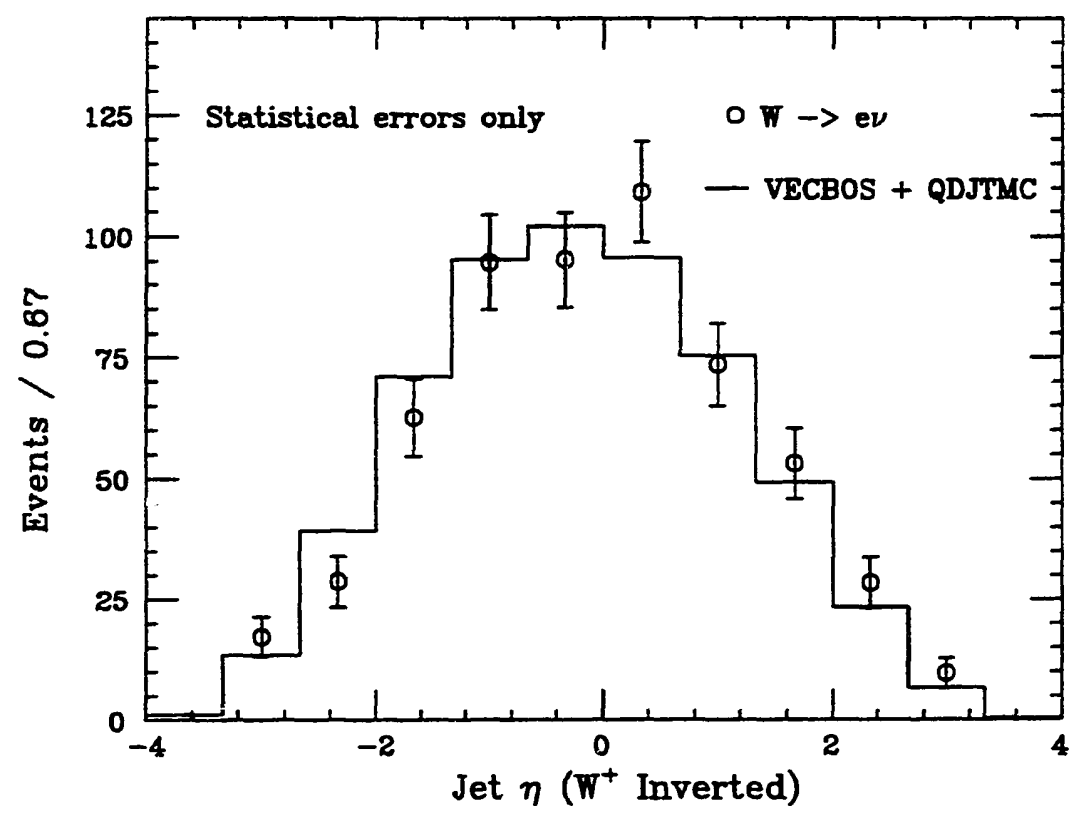

Figure 6.7: Jet pseudo-rapidity, in the $W+$ Jet samples, compared to the VECBOSQDJTMC prediction. $\mathrm{W}^{+}$events are inverted in this distribution. 


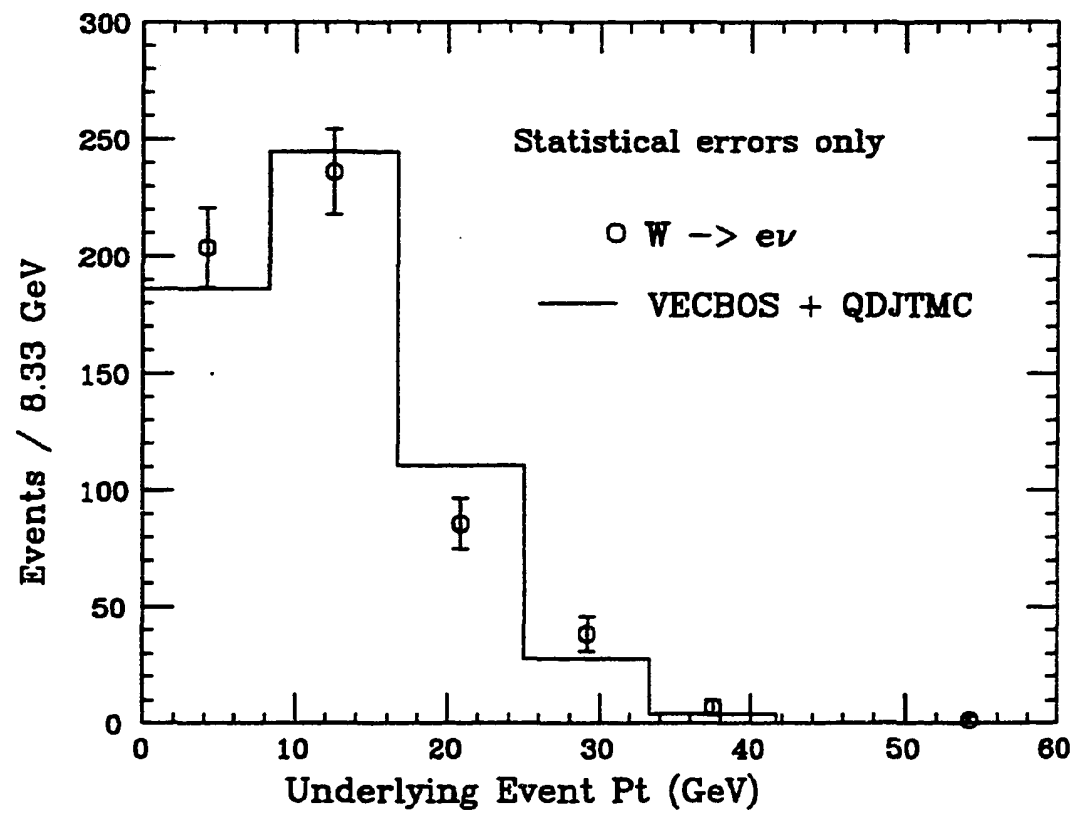

Figure 6.8: Transverse momentum of the underlying-event, in the $\mathrm{W}+$ Jet sample, compared to the VECBOS-QDJTMC prediction.

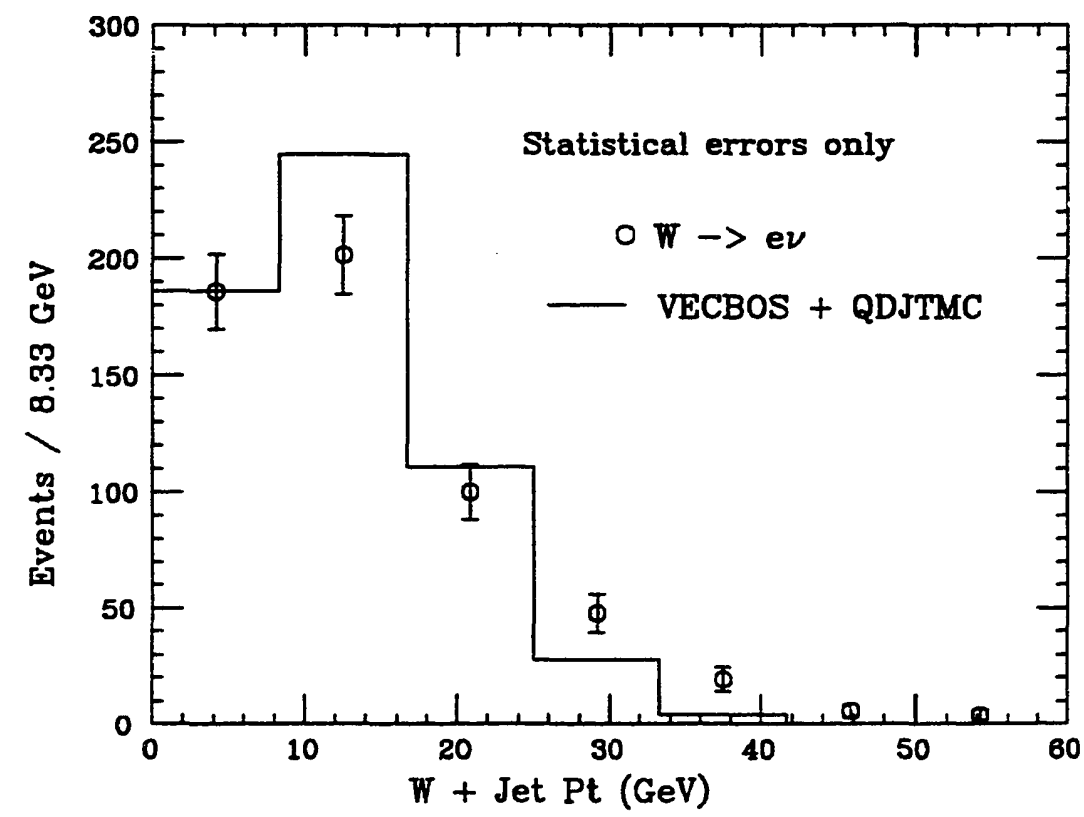

Figure 6.9: Transverse momentum of the $\mathrm{W}+$ jet system, compared to the VECBOSQDJTMC prediction. 


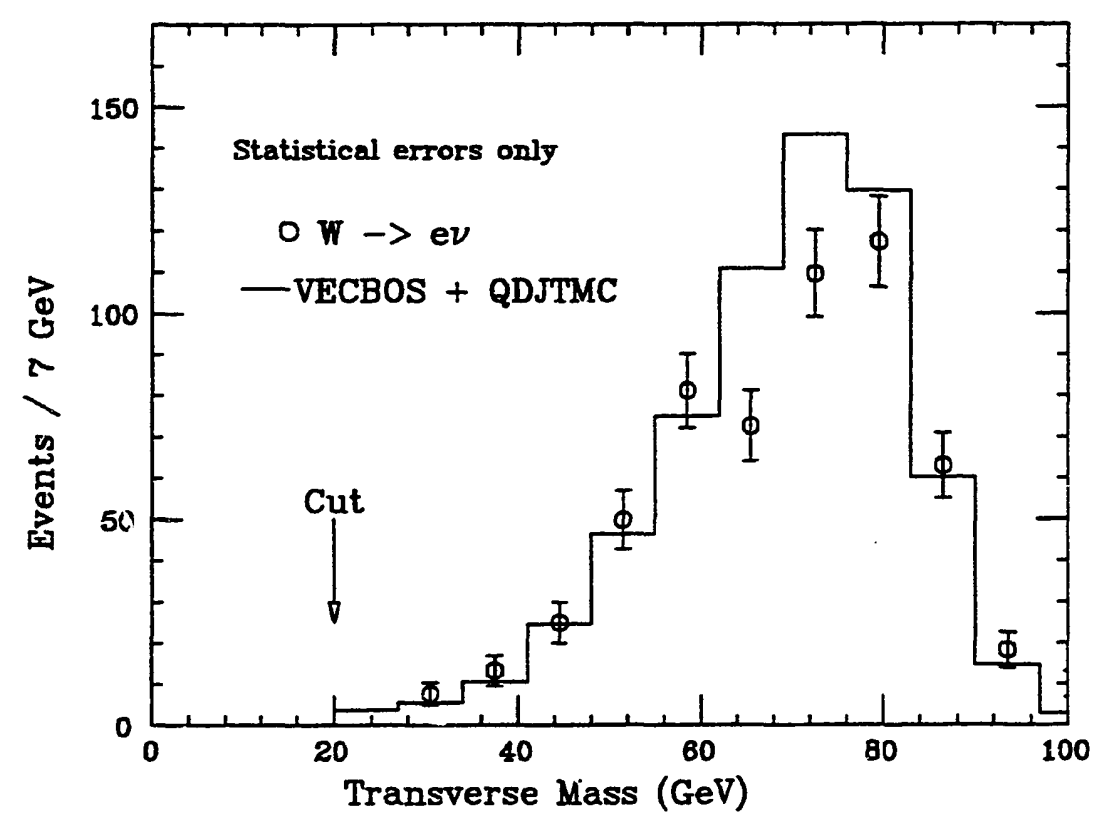

Figure 6.10: $\mathrm{W}$ transverse mass, in the $\mathrm{W}+$ Jet sample, compared to the VECBOSQDJTMC prediction.

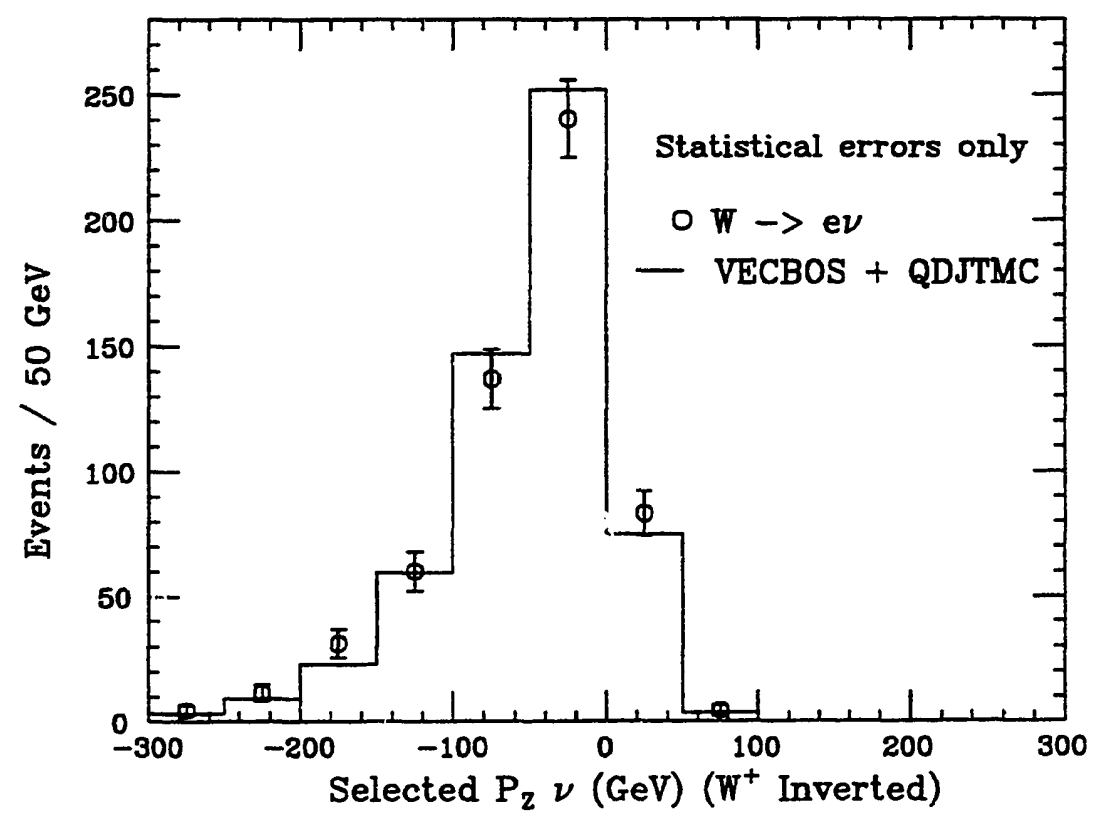

Figure 6.11: The selected solution for the $\mathrm{z}$-momentum of the neutrino, in the $\mathrm{W}+$ Jet sample, compared to the VECBOS-QDJTMC prediction. $\mathrm{W}^{+}$events are inverted in this distribution. 


\section{Chapter 7}

\section{Analysis}

The previous chapters have defined the $W+$ Jet sample, which contains 411 events (of which 31 are estimated to be background; discussed in Chapter 8 ). This chapter describes the analysis method used to extract the selected $\cos \theta^{*}$ distribution from these events. Much of what is presented in this chapter has already been discussed in Chapter 3; however, the presentation here is more detailed and discusses the properties of the CDF detector (which was avoided, if possible, in Chapter 3).

\subsection{W Mass Constraint}

The first step in the analysis of the $W+$ jet events is the determination of the $z$-component of the neutrino momentum $\left(\mathrm{P}_{z}^{\nu}\right)$. As discussed in section 3.2.1, if the invariant mass of the electron-neutrino pair is required to be $80 \mathrm{GeV}$, then $\mathrm{P}_{z}^{\nu}$ is constrained to two possible values:

$$
\mathrm{P}_{z}^{\nu}=\frac{1}{2 P_{t e}^{2}}\left[A P_{z e} \pm E_{e} \sqrt{A^{2}-4 P_{t e}^{2} P_{t \nu}^{2}}\right]
$$

where

$$
A=\mathrm{M}_{W}^{2}+P_{t W}^{2}-P_{t e}^{2}-P_{t \nu}^{2}
$$

$P_{t W}, F_{t e}$, and $P_{t \nu}$ are the transverse momenta of the W, electron, and neutrino respectively, and $P_{z e}$ and $E_{e}$ are the electron z momentum and energy. $\mathrm{M}_{W}$ is set to $80 \mathrm{GeV}$ (the current CDF measurement of the W mass[18]).

\subsubsection{Events Failing the W Mass Constraint}

Some of the events will not be constrainable to $80 \mathrm{GeV}$, that is, they will produce complex solutions for $\mathrm{P}_{z}^{\nu}$. The VECBOS Monte Carlo in combination with the QDJTMC detector simulation predicts that $21 \%$ of the events will fail the constraint. 
The fraction failing the constraint in the CDF data is $24 \pm \% \%$.

It is easier to understand the conditions under which the events cannot be constrained if equation 7.1 is rewritten in terms of the transverse-mass. The transverse-mass is defined to be the invariant mass of the electron and neutrino pair that results when the $z$-components of both particles are taken to be zero. That is, the transverse-mass $\left(M_{t}\right)$ is:

$$
\mathrm{M}_{t}=\sqrt{2 P_{t e} P_{t \nu}\left[1-\cos \Delta \phi_{e \nu}\right]},
$$

where $\Delta \phi_{e \nu}$ is the angle between the electron and the neutrino in the transverse plane (the $x-y$ plane). Expressed in terms of $M_{t}$ the part of equation 7.1 inside the radical becomes:

$$
A^{2}-4 P_{t e}^{2} P_{t \nu}^{2}=\left(\mathrm{M}_{W}^{2}-\mathrm{M}_{t}^{2}\right)\left[\mathrm{M}_{W}^{2}+2 P_{t e} P_{t \nu}\left(1+\cos \Delta \phi_{e \nu}\right)\right] .
$$

The quantity can only be negative if the transverse-mass is greater than $M_{W}$ (which is $80 \mathrm{GeV}$ ). Therefore, the events will fail the mass constraint if, and only if, the transverse-mass is greater than $80 \mathrm{GeV}$.

Figure 5.8 shows the transverse-mass distribution for the data compared with the Monte Carlo prediction. The distribution peaks near $80 \mathrm{GeV}$ and has a small tail that is smeared above $80 \mathrm{GeV}$. There are two factors contributing to the tail above $80 \mathrm{GeV}$. The dominant effect is the detector energy resolution on the electron and the neutrino. This will cause the sharp drop off above $80 \mathrm{GeV}$ to become more smeared out (which is what is seen). The other effect is the natural width of the $\mathrm{W}$ boson itself; that is, some events will be produced in which the W's mass is greater than $80 \mathrm{GeV}$. If there were no detector resolution effects, the Monte Carlo predicts that the fraction of events with $\mathrm{M}_{t}>80 \mathrm{GeV}$ would be about half of what it is with the detector resolution.

The method used for dealing with these constraint failures is to force them to constrain by using the value of the transverse-mass for $\mathrm{M}_{W}$ instead of using $80 \mathrm{GeV}$. Since $\mathrm{M}_{t}$ is, typically, only smeared a few $\mathrm{GeV}$ above 80 , this treatment of the events is only very slightly discontinuous from the treatment of the events that can be constrained to $80 \mathrm{GeV}$.

Another method was considered. The constraint failures could be removed from the event sample. This method not only reduces the statistical power of the $\cos \theta_{s}^{*}$ measurement (due to the reduced number of events), but it also causes a bias in the $\cos \theta_{s}^{*}$ distribution. One reason that this is a bias is that throwing these events out means throwing out events in which the detector simulation has caused $M_{t}$ to fluctuate high, but keeping events in which $M_{t}$ has fluctuated low.

In section 7.8 , the residual acceptance in the $\cos \theta_{s}^{*}$ distribution will be examined using both methods for the treatment of the constraint failures. Retaining the events and constraining them to $M_{W}=M_{t}$ results in a flat $\cos \theta_{s}^{*}$ acceptance, whereas throwing out the events causes a clear bias in the $\cos \theta_{s}^{*}$ distribution. 
In the events constrained to $\mathrm{M}_{W}=\mathrm{M}_{t}$, the two solutions for $\mathrm{P}_{z}^{\nu}$ will be identical (because the radical in equation 7.1 has been forced to be zero). Even without detector smearing, these events would have a transverse-mass close to $80 \mathrm{GeV}$ (or above); therefore, they would have two solutions for $\mathrm{P}_{z}^{\nu}$ that were close together anyway. Figure 7.1 shows the difference between the true and false $\mathrm{P}_{z}^{\nu}$ solutions for Monte Carlo events before detector simulation. The narrower peak represents the events that failed the $80 \mathrm{GeV}$ mass constraint after they were detector simulated (however, the plot is showing pre-simulated quantities). The wider peak represents those events that were successfully constrained to $80 \mathrm{GeV}$ after detector simulation. The plot shows that the events which fail to constrain to $80 \mathrm{GeV}$ do indeed have two solutions that are almost identical; therefore it is not a problem (or a bias) to force them to constrain in such a way that the two solutions are identical.

\section{2 $\mathrm{W}^{+}$Event Inversion}

Many of the variables that describe the $W+$ Jet system have asymmetric distributions (e.g. $\left.\cos \theta^{*}\right)$. These asymmetries are "washed out" when $\mathrm{W}^{+}$and $\mathrm{W}^{-}$events are added together. These asymmetries can be preserved if the distributions are inverted (for example, $\cos \theta^{*} \rightarrow-\cos \theta^{*}$ ) for one sign of the $\mathrm{W}$ charge ${ }^{1}$. From this point on in this chapter, $\mathrm{W}^{+}$events have been inverted. Instead of inverting each distribution as it occurs, the events are inverted at this point in the analysis. Inverting the events means inverting the $z$-component of the momentum for each particle (e.g. $\mathrm{P}_{z}^{\nu} \rightarrow-\mathrm{P}_{z}^{\nu}$ ) in the $\mathrm{W}^{+}$events, and treating them in the same way as the $\mathrm{W}^{-}$events. The reader will be reminded, occasionally, that the $\mathrm{W}^{+}$events have been inverted.

\subsection{Solution Selection}

One of the two event solutions must now be chosen as the "selected" solution. One might hope that, in some fraction of the events, one of the event solutions would represent an unphysical situation (e.g. a solution in which the total energy is greater than the beam energy-1.8 TeV). Unfortunately, this almost never happens in $1.8 \mathrm{TeV}$ proton-antiproton collisions. The VECBOS Monte Carlo, with the detector simulation, predicts that $0.0003 \%$ of the events will have an unphysical solution. None of the CDF data events has an unphysical solution. As discussed in section 3.2 .2 , the fact that the $W$ is expected to be strongly polarized

\footnotetext{
${ }^{1}$ This is true because these events are expected to be invariant under the application of the $\mathrm{CP}$ (charge-conjugation and parity) operator. Inverting $\mathrm{W}^{+}$events and then treating them as $\mathrm{W}^{-}$events is equivalent to the application of CP).
} 


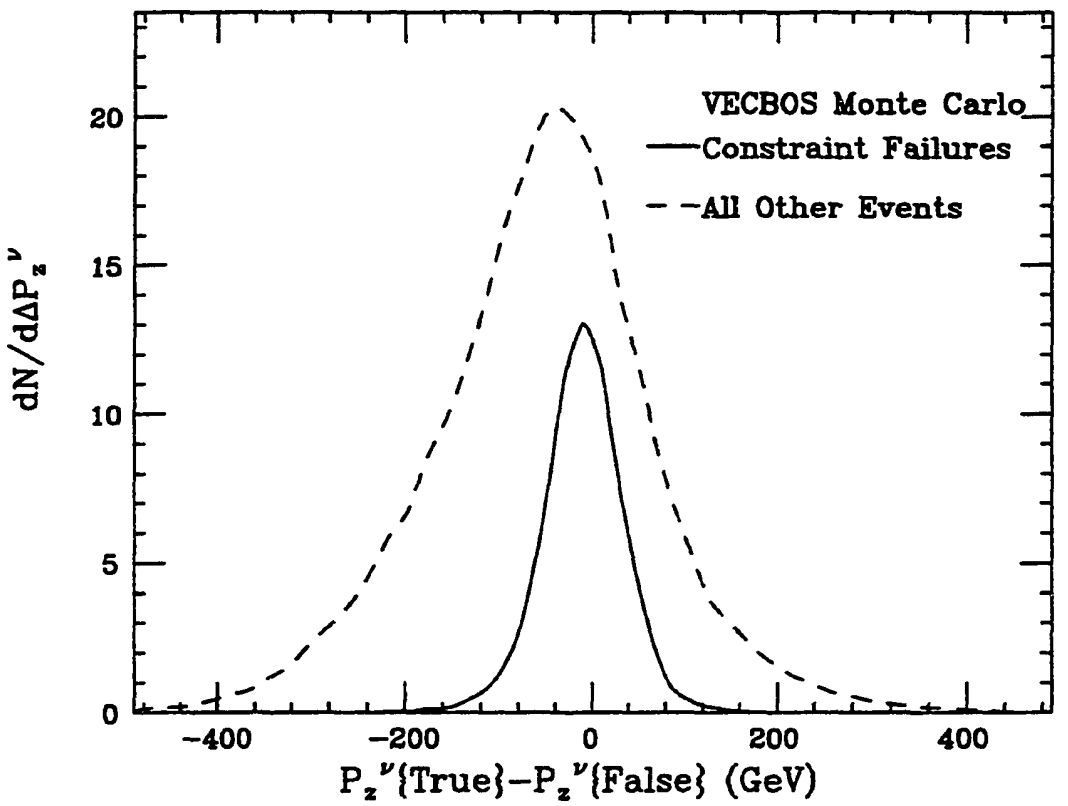

Figure 7.1: True minus false values of the neutrino's z-momentum as generated by the VEcBos Monte Carlo. The narrower (solid) curve shows the events that fail to constrain to $80 \mathrm{GeV}$ after the detector simulation. The wider (dashed) curve shows all events except those in the narrower curve. 
in the $\mathrm{W}+$ jet events is used to choose one of the even ii solutions as being more likely.

First, a Lorentz boost is made into the rest frame of the $W$. The boost is done along the $\mathrm{z}$ direction first, and then in the transverse direction (this is the Collins-Soper prescription described in section 3.1.1). Then, in the W rest frame, the variable $\cos \alpha_{C S}$ is defined as the cosine of the angle between the electron and the positive $z$-axis (note that this is the $z$-axis in the rest frame of the $\mathrm{W}$, and not the $\mathrm{z}$-axis in the lab frame, see section 3.1.3). The $\mathrm{W}$ tends to be polarized along the negative $\mathrm{z}$-axis in this frame and therefore the distribution of $\cos \alpha_{C S}$ will be peaked near 1.0 (see figure 3.7). Note that $\cos \alpha_{C S}$ has been inverted for $\mathrm{W}^{+}$events as a result of the $\mathrm{W}^{+}$event inversion described in section 7.2.

The "selected" solution is defined to be the solution with the larger value of $\cos \alpha_{C S}$. This choice is predicted to be correct, $b ;$ the VECBOS-QDJTMC Monte Carlo, in $73 \%$ of the events.

From this point on in the analysis, only the selected solution for dual-valued variables will be used. For example, when a cut is applied to $M^{*}$, the selected solution will be used for the cut.

\subsection{Center-of-mass Variables}

The next step in the analysis is to boost into the center-of-mass frame of the $\mathrm{W}+$ jet system. This is done, again, by boosting first along the z-axis and then in the transverse plane. The center-of-mass variables were defined in section 3.1.2. The boost, along the $\mathrm{z}$-axis, from the lab frame, expressed as the change in rapidity is $Y_{\text {Boost }}$. In the center-of-mass frame $\cos \theta^{*}$ is defined to be the cosine of the angle between the $W$ and positive $z$-axis (recall that the $W^{+}$events have been inverted), and $M_{s}^{*}$ is the total energy of the $W+$ jet system.

\section{5 $\operatorname{Cos} \theta_{\mathrm{s}}^{*}$ Region Measured}

In this thesis, the selected $\cos \theta^{*}\left(\cos \theta_{s}^{*}\right)$ distribution will be measured between -0.9 and 0.9 . This value was chosen by using the Monte Carlo events to optimize the cut. Figure 7.2 shows the statistical significance of the largest bin in the $\cos \theta_{s}^{*}$ distribution as a function of the maximum $\cos \theta_{s}^{*}$ cut. The statistical significance of the bin is defined to be the ratio of the statistical error on the bin over the number of events in the bin. The largest bin was defined to cover $10 \%$ of the allowed region (e.g. for a 0.9 cut on $\cos \theta_{s}^{*}$ the lower bin would go from -0.9 to -0.72 ). The error on the bin included the uncertainty from normalizing the $\cos \theta_{s}^{*}$ distribution to have an average value of 1.0 in the inner $60 \%$ of the allowed region 
(the normalization, and its effect on the errors, will be discussed in more detail in section 10.2).

The plot shows less statistical significance at smaller values of the $\cos \theta_{s}^{*}$ cut, because the $\cos \theta_{s}^{*}$ distribution becomes less peaked as the cut is made smaller. The normalization region is also becoming smaller (which makes the statistical errors larger). The plot shows less statistical significance again at large values of the cut, because the larger the $\cos \theta_{s}^{*}$ cut gets the larger the $\mathrm{M}^{*}$ cut gets, which throws out more of the events (the $\mathrm{M}_{s}^{*}$ cut will be described in section 7.7).

The plot shows that a $\cos \theta_{s}^{*}$ cut somewhere between 0.8 and 0.9 would be best, so the cut was chosen to be 0.9 in order to measure as much of the $\cos \theta_{s}^{*}$ distribution as possible.

\section{6 $Y_{\text {Boost }}^{s}$ Cut}

A cut is placed on the z-component of the boost from the lab frame into the $W$ + jet center-of-mass frame:

$$
\left|Y_{\text {Boost }}^{s}\right|<1.5 .
$$

This corresponds to a cut of 0.905 times the speed of light on the boost from the lab frame into the center-of-mass frame. This cut serves two purposes. In combination with the maximum $\cos \theta_{s}^{*}$ cut, it serves to keep the jet well within the CDF calorimetry (it is not desirable to have to express cuts in terms of detector coordinates). The second purpose of the $Y_{\text {Boost }}^{s}$ cut is that it helps to remove events in which the electron is not in the central calorimeter (recall that there is a cut on the electron to be central in the lab frame: $\left|\eta_{\text {Lab }}\right|<0.95$, see section 5.1.2). This helps to reduce the acceptance effect in the $\cos \theta_{s}^{*}$ distribution caused by the central-electron requirement. It also helps to reduce the uncertainties on this acceptance due to the uncertainties in the parton distribution functions (see section 9.3).

\section{7 $\quad \mathrm{M}_{s}^{*}$ Cut}

As discussed in section 3.1 .2 , the $\mathrm{P}_{t}$ cut of $15 \mathrm{GeV}$ on the jet flattens the $\cos \theta_{s}^{*}$ distribution by preferentially throwing out events at large values of $\left|\cos \theta_{s}^{*}\right|$. The $\cos \theta_{s}^{*}$ distribution can be restored by making a cut on the total energy of the $W$ + jet system, $M_{s}^{*}$. The variables $M^{*}$ and $\cos \theta^{*}$ are approximately independent (that is, in the matrix elements for $\mathrm{W}+$ jet production), and so cutting on $\mathrm{M}_{s}^{*}$ is an excellent way to remove this acceptance effect.

With the 0.9 cut on $\left|\cos \theta_{s}^{*}\right|$, and a cut of $121.5 \mathrm{GeV}$ on $\mathrm{M}_{s}^{*}$ (see equation 3.1), the jet $P_{t}$ cannot be less than $15 \mathrm{GeV}$. This removes the jet $P_{t}$ acceptance effect 


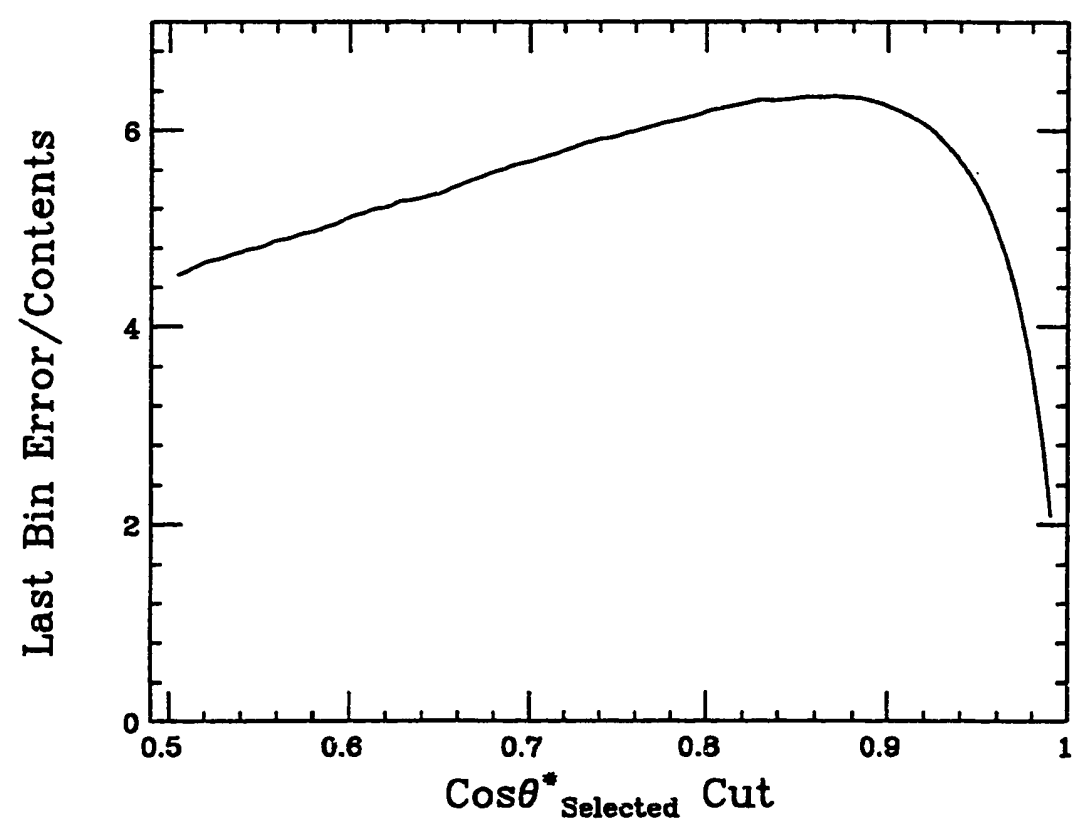

Figure 7.2: The statistical significance of the last bin in $\cos \theta_{s}^{*}$ as a function of the maximum cut on $\cos \theta_{s}^{*}$. The normalization region is taken to be within $60 \%$ of the cut. The last bin is defined to extend from the lower cut and cover $10 \%$ of the allowed region. 
on $\cos \theta_{s}^{*}$.

By cutting on $M_{s}^{*}$, the minimum allowed jet $P_{t}$ will be a function of $\cos \theta_{s}^{*}$. Figure 7.3 shows the minimum $P_{t}$ that a jet can have and still pass the $M_{s}^{*}$ cut, as a function of $\cos \theta_{s}^{*}$. Another advantage of the $M_{s}^{*}$ cut is that most of the low- $\mathrm{P}_{t}$ jets are removed. Figure 7.4 shows the jet $P_{t}$ spectrum before and after the $M_{s}^{*}$ cut is applied. The figure shows that most of the jets with $P_{t}$ less than $25 \mathrm{GeV}$ are removed frum the sample. This is an advantage because many of the systematic uncertainties associated with low-energy jets are done-away with. This is also an advantage because the steeply falling $\mathrm{P}_{t}$ spectrum is flattened, so the distribution will not be changed as much by detector resolution effects.

\subsection{Residual Detector Effects and $\operatorname{Cos} \theta_{\mathrm{s}}^{*}$ Reso- lution}

All of the cuts placed on the data have now been described. The final event sample contains 165 events. Many of the cuts have been applied in order to have an acceptance that is flat in $\cos \theta_{s}^{*}$ and in order to make the analysis as insensitive to the detector smearing effects as possible.

In order to look for any residual effects of the CDF detector on the $\cos \theta_{s}^{*}$ distribution (i.e. through acceptance effects, or through detector resolution effects), the $\cos \theta_{s}^{*}$ distributions before and after the detector simulation will be compared.

The $\cos \theta_{s}^{*}$ distribution with detector simulation was made by generating VECBOS events and running then through the QDJTMC detector simulation. The events were then analysed in the same way as the data.

In order to make the $\cos \theta_{s}^{*}$ distribution without the detector simulation, VECBOS events were generated and used directly to make the $\cos \theta_{s}^{*}$ distribution. The following cuts were applied to the events in order to compare them with the detector simulated Monte Carlo events:

- Electron $\mathrm{P}_{t}>20 \mathrm{GeV}$

- Neutrino $\mathrm{P}_{t}>20 \mathrm{GeV}$

- Electron $|\eta|<0.95$

- Jet $P_{t}>15 \mathrm{GeV}$

- $\Delta R($ electron - jet $)>0.9$

- W Transverse Mass $>20 \mathrm{GeV}$

- $\left|Y_{\text {Boost }}^{s}\right|<1.5$ 


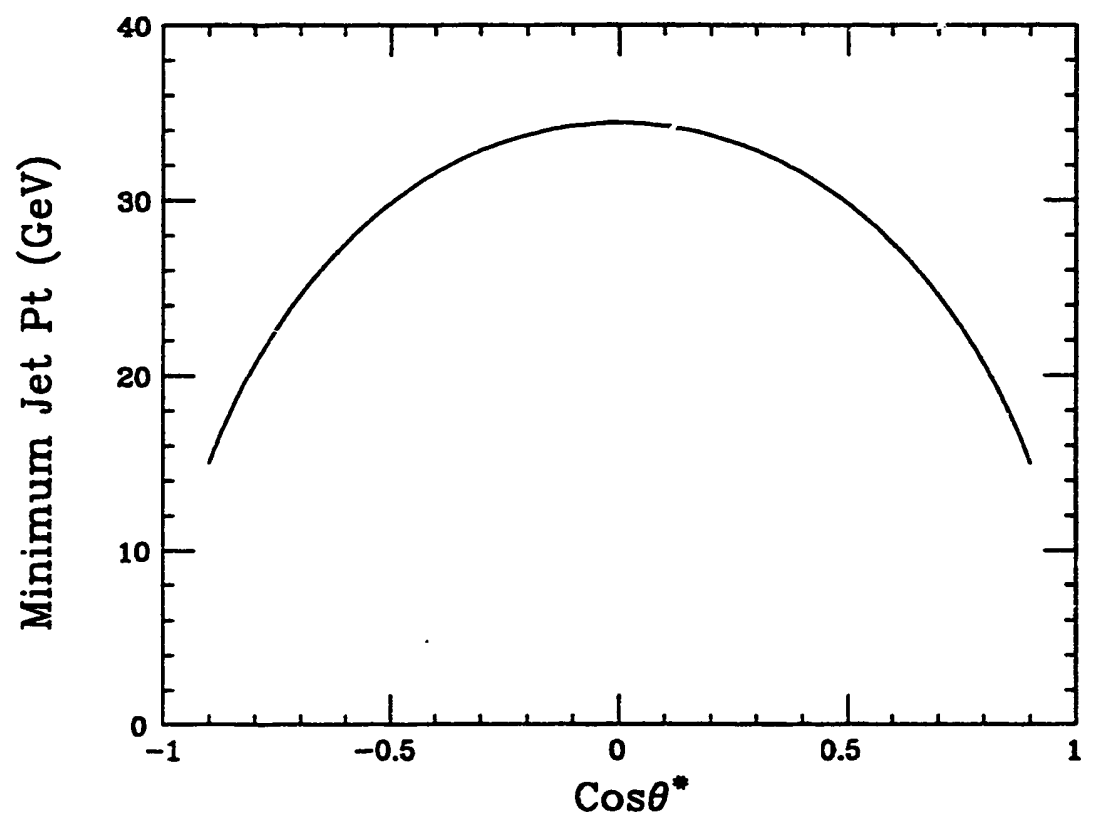

Figure 7.3: The minimum jet $P_{t}$, as a function of $\cos \theta^{*}$, that will pass the $M^{*}$ cut. Inverting equation 3.1 gives the analytic expression used to make this curve: $\mathbf{P}_{t_{\min }}=$ $\frac{1}{2}\left(M_{\text {cut }}^{* 2}-M_{W}^{2}\right) \sin \left(\theta^{*}\right)$. 


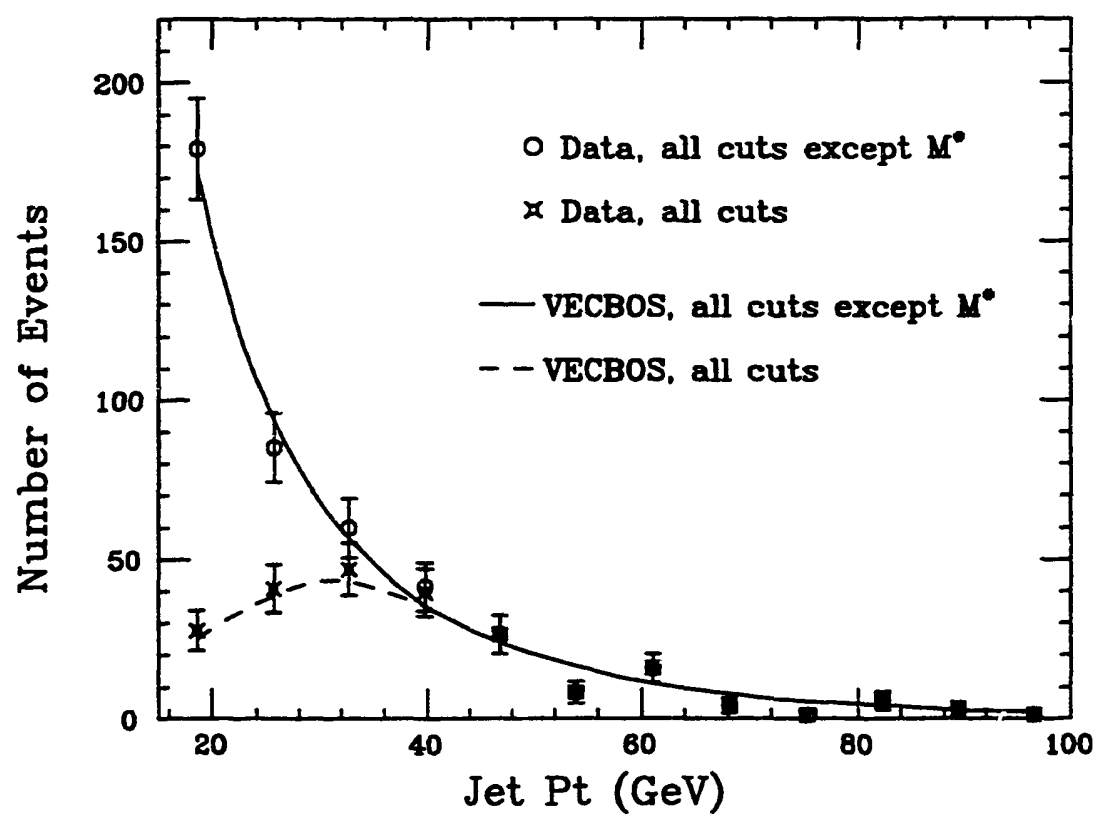

Figure 7.4: The $\mathrm{P}_{t}$ spectrum of the jet. The circles are the CDF data with all cuts applied except the $M_{s}^{*}$ cut. The stars are the data with all cuts applied. The solid curve is the Monte Carlo prediction without the $M_{s}^{*}$ cut, and the dashed curve has the $M_{s}^{*}$ cut applied. 


$$
\begin{aligned}
& \text { - } \mathrm{M}_{s}^{*}>121.5 \mathrm{GeV} \\
& \text { - }\left|\cos \theta_{s}^{*}\right|<0.9
\end{aligned}
$$

The z-component of the neutrino momentum was obtained, for these events, by constraining the neutrino to the $\mathrm{W}$ boson mass generated by the Monte Carlo. This means that one of the solutions was exactly equal to the true $\mathrm{P}_{z}^{\nu}$ generated by the Monte Carlo; however, the other solution was needed because it was the selected solution in roughly $30 \%$ of the events. Using the known $\mathrm{W}$ boson mass instead of constraining to a fixed $80 \mathrm{GeV}$ is done in order to make it simple to compare other theories with the results of this analysis. The effect of using the fixed $80 \mathrm{GeV}$ constraint is considered to be included in the "detector effects" $\mathrm{nd}$ is a small resolution effect on $\cos \theta_{s}^{*}$ ).

Figure 7.5 shows the ratio of the $\cos \theta_{s}^{*}$ distribution with the detector simulation to the $\cos \theta_{s}^{*}$ distribution without detector simulation. This plot is flat, within the statistical errors of the Monte Carlo sample, which demonstrates that any residual detector effects are small.

The average value of the ratio is 1.06 , which means that the detector simulation allows $6 \%$ more events to smear into the sample. This can be understood by looking at the $\mathrm{M}^{*}$ distribution in figure 3.4 . The $\mathrm{M}^{*}$ distribution is falling rapidly near the $121.5 \mathrm{GeV}$ cut, so the effect of detector energy smearing will be to smear more events up from below the cut than it smears down from above the cut. The net effect is a slight increase in the number of events. However, the shape of the $\cos \theta_{s}^{*}$ distribution does not change rapidly with $\mathrm{M}^{*}$ (i.e. they are relatively independent variables); therefore, the smearing of the $\mathrm{M}^{*}$ events into the sample does not change the shape of $\cos \theta_{s}^{*}$. Since the final $\cos \theta_{s}^{*}$ distribution will be normalized, between -0.6 and 0.6 , the slight increase in the total number of events has no effect on the final result.

As was promised in section 7.1.1, figure 7.6 shows the residual detector effects on the $\cos \theta_{s}^{*}$ distribution when events unconstrainable to $80 \mathrm{GeV}$ are rejected. Throwing away the constraint failures clearly distorts the $\cos \theta_{s}^{*}$ distribution.

The residual detector effects include the measurement resolution of $\cos \theta_{s}^{*}$. Figure 7.7 shows a scatter plot of the detector-simulated value of $\cos \theta_{s}^{*}$ versus the generated value of $\cos \theta_{s}^{*}$. The plot only contains the events which passed the analysis cuts in both the simulated and generated event samples. Figure 7.8 shows the root-mean-square resolution on $\cos \theta_{s}^{*}$. The dashed line shows the mathematical relation $d \cos \theta^{*} / d \eta$, which, because it is similar to the Monte Carlo prediction, demonstrates that the resolution is approximately flat in $\eta$. The $\cos \theta_{s}^{*}$ resolution is slightly smaller than the bin width used in the final $\cos \theta_{s}^{*}$ plots. The resolution has two, approximately equal, components: the resolution of the CDF detector, and the natural width of the $\mathrm{W}$ boson (which was discussed in section 3.2.1). 


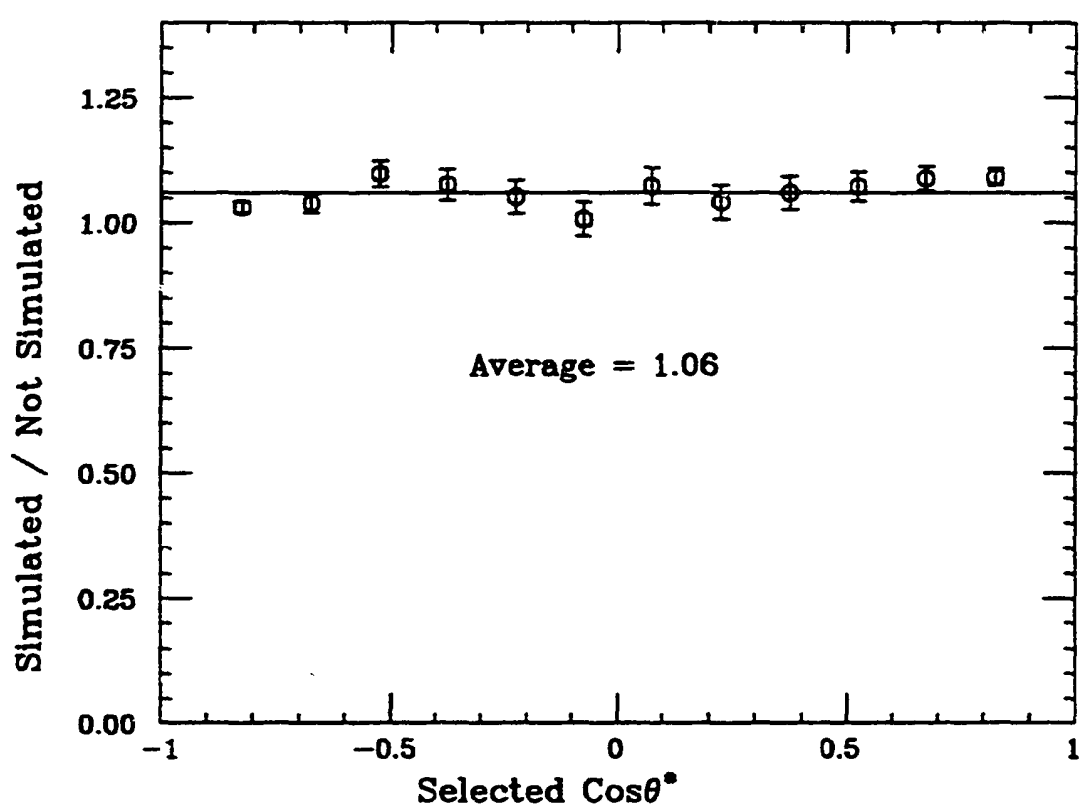

Figure 7.5: Residual detector effects in $\cos \theta_{s}^{*}$. The curve shows the ratio of the $\cos \theta_{s}^{*}$ distribution after QDJTMC simulation to that before the simulation. The flat line is drawn at the average value of the points. 


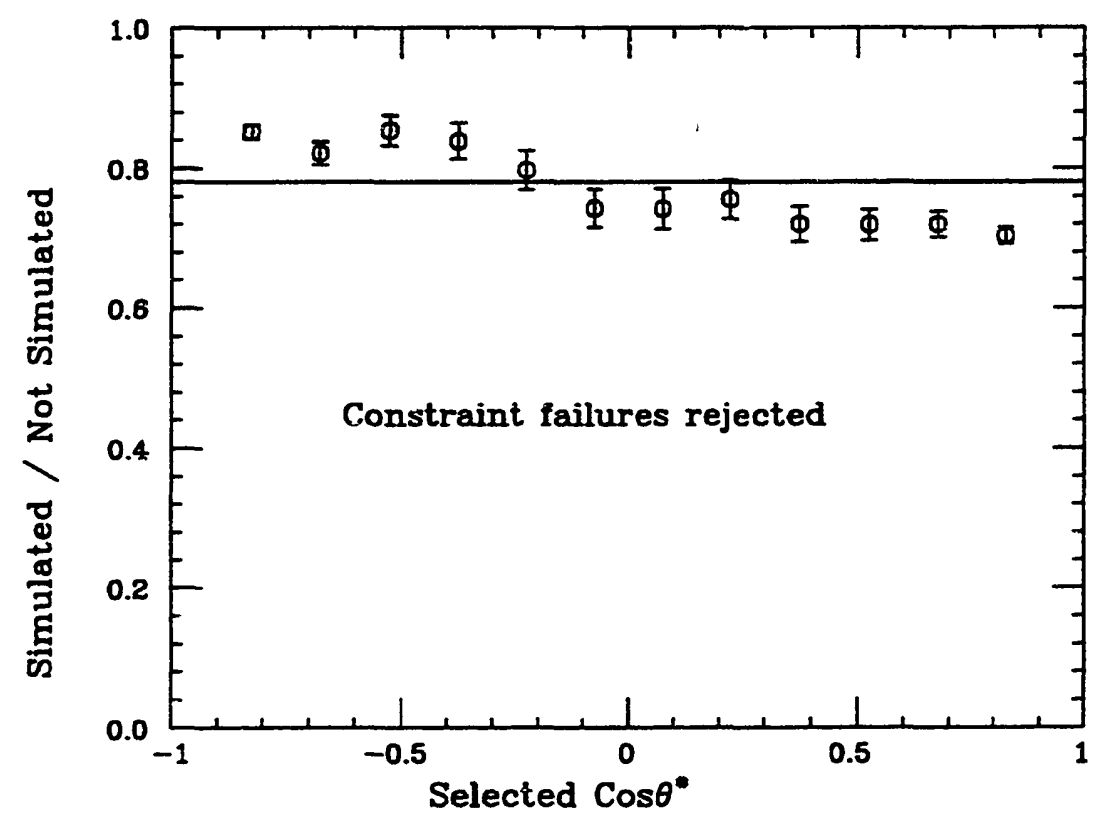

Figure 7.6: Residual detector effects in $\cos \theta_{s}^{*}$. The curve shows the ratio of the $\cos \theta_{s}^{*}$ distribution after QDJTMC simulation to that before the simulation. In making this plot events were discarded after simulation if they failed to constrain to an $80 \mathrm{GeV}$ W mass. The fiat line is drawn at the average value of the points. 


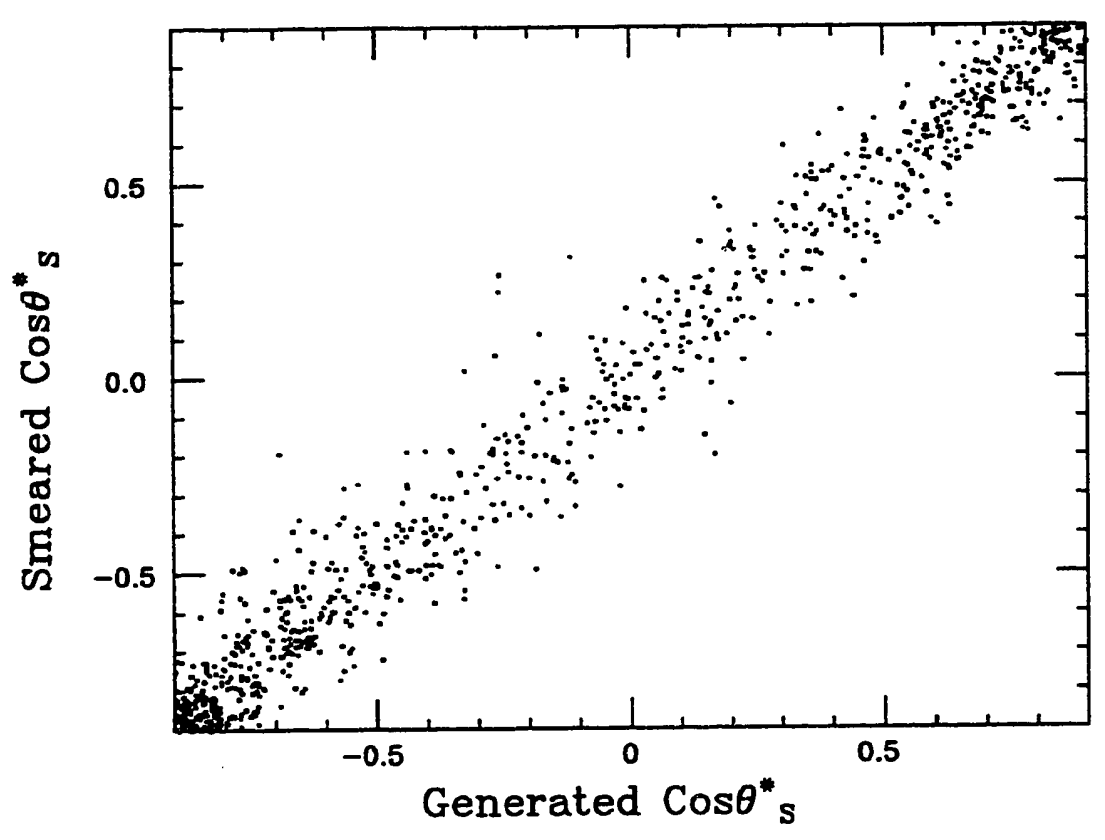

Figure 7.7: A scatter plot of selected $\cos \theta^{*}$. The $\mathrm{x}$-axis is the generated value of $\cos \theta_{s}^{*}$, and the $y$-axis is $\cos \theta_{s}^{*}$ after detector simulation. Events were entered in this plot if they passed all of the analysis cuts for both the smeared and generated samples. 


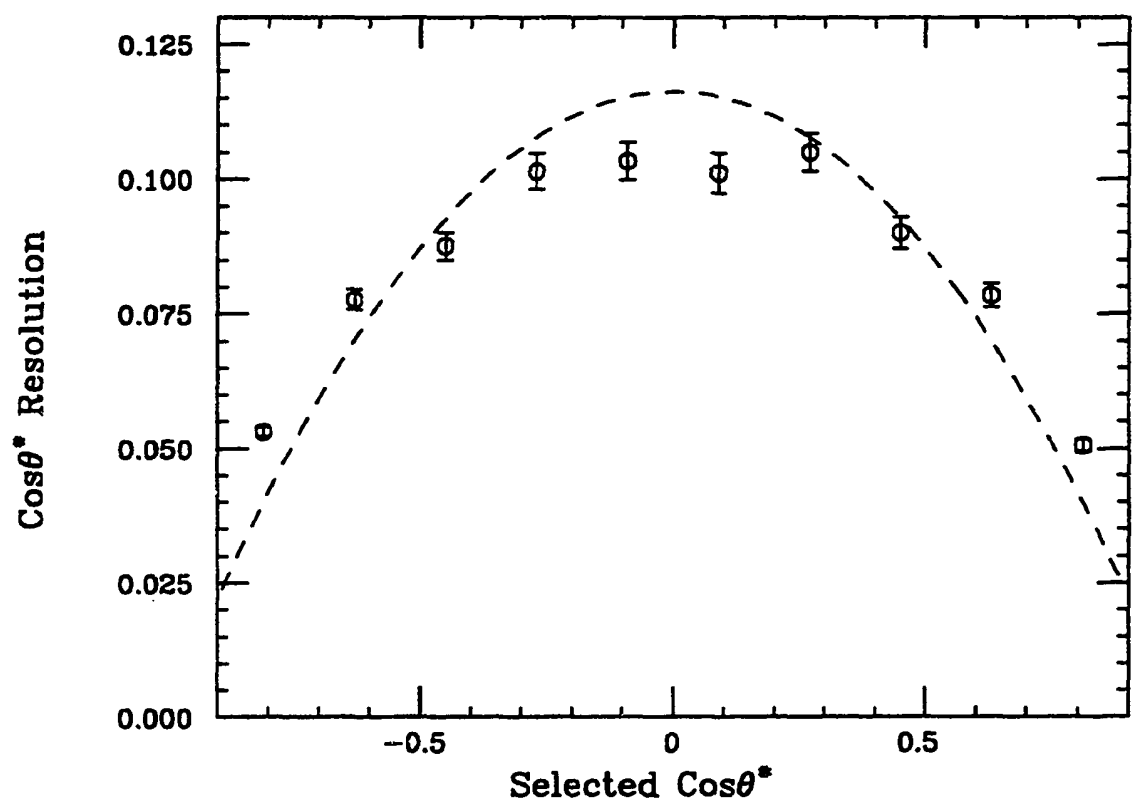

Figure 7.8: The root-mean-square resolution of $\cos \theta_{s}^{*}$ as determined from figure 7.7. The dashed line shows the mathematical relation $d \cos \theta^{*} / d \eta$, which demonstrates that the resolution is approximately flat in $\eta$. 
In the following chapters, the backgrounds in the event sample will be subtracted from the $\cos \theta_{s}^{*}$ distribution, and the systematic uncertainties on the measurement will be discussed. In Chapter 10 the final $\cos \theta_{s}^{*}$ distribution will be compared to the theoretical predictions. 


\section{Chapter 8}

\section{Backgrounds}

The identification cuts placed on the electron along with the kinematic cuts on the events provide a sample of $\mathrm{W} \rightarrow e \nu$ events that has very little background contamination. There are, however, several sources of background events that are expected to remain in the sample. Each of the expected backgrounds is discussed in this chapter.

For each background, an estimate is made of the number of events in the $\mathrm{W}$ + Jet sample. The background contribution is then subtracted from the selected $\cos \theta^{*}$ distribution, using the $\cos \theta_{s}^{*}$ distribution predicted for each background.

It is important to note that most of the backgrounds considered have a $\cos \theta_{s}^{*}$ distribution that is very similar to the $\cos \theta_{s}^{*}$ for the signal events. Systematic uncertainties due to background subtraction are estimated by varying the number of events subtracted (see section 9.2 ). The final $\cos \theta_{s}^{*}$ plot has an arbitrary normalization (see section 3.5); therefore, the background subtraction will only have a significant effect on the final $\cos \theta_{s}^{*}$ plot if the background shape is significantly different than that of the signal events.

\subsection{Electroweak Backgrounds}

One source of backgrounds to the $\mathrm{W}+$ Jet signal events are the production of $\mathrm{W}$ and $\mathrm{Z}$ bosons. The $\mathrm{W}$ 's produced can decay into tau leptons which can then decay into an electron; these events are very similar to the direct $W \rightarrow e \nu$ decays. When Z's decay into $e^{+} e^{-}$or $\tau^{+} \tau^{-}$they can produce events that mimic $W \rightarrow e \nu$ decays; this requires that the other lepton from the $\mathrm{Z}$ escape the detector unmeasured.

Each of these three electroweak backgrounds are discussed below. 


\subsection{1 $W \rightarrow \tau \nu \rightarrow e \nu \nu \nu$}

W bosons that decay into a tau-which subsequently decays into an electron and neutrinos-are a background to the direct $W \rightarrow e \nu$ decays. The electron quality cuts do not remove these events because they produce a real electron.

$\mathrm{W}$ boson plus one jet events were generated with the Pythia [23] Monte Carlo program, which was discussed in section 6.2.1. The $W$ was forced to decay to a tau, and the tau was forced to decay to $e \overline{\nu_{e}} \nu_{\tau}$. A tau resulting from the decay of a $\mathrm{W}$ will be predominately in a single helicity state (negative helicity for a $\tau^{-}$) and this will affect the distribution of the electron from the tau decay. PYTHIA does not include this effect, so, after each event was generated by PYTHIA, the decay products of the tau were rotated-in the tau rest frame-to get the correct distribution. In the rest frame of the tau the distribution should be

$$
\frac{d \sigma}{d \Omega}=1+\frac{1}{3} \cos \theta
$$

where $\theta$ is the angle between the electron (positron) and the direction of the original tau momenta (the axis of the helicity).

The events were then run through the QFL detector simulation (see section 6.2.2) and through the same analysis as the signal events. Figure 8.1 shows the distribution of $\cos \theta_{s}^{*}$ for these events and compares it to the $W \rightarrow e \nu$ signal shape. In order to estimate the number of these events in the final data sample, $\mathrm{W}+$ jet events were generated with Pythia in which the $\mathrm{W}$ decays to $e \nu$. These signal Monte Carlo events were then combined with all of the background predictions-electroweak and QCD (described in the next section). The fraction of this total Monte Carlo prediction represented by the $W \rightarrow \tau \nu$ prediction forms the estimate for the fraction of $W \rightarrow \tau \nu$ events in the CDF data sample.

Based on this method, $3.5 \%$ of the final event sample is $W \rightarrow \tau \nu \rightarrow e \nu \nu \nu$.

\subsection{2 $Z \rightarrow \mathrm{e}^{+} \mathrm{e}^{-}$}

Another background to the $\mathrm{W}+$ jet signal are $\mathrm{Z}+$ Jet events in which the $\mathrm{Z}$ decays into an electron and a positron and one of these leptons is undermeasured by the detector. An electron can be undermeasured if it goes into a section of the detector that is not well instrumented (a boundary between two detector sections), or if it exits very close to the beamline.

This background was estimated by generating $\mathrm{Z}+$ jet events with Pythia in which the $Z$ was forced to decay to electrons. The events were then run through the QFL detector simulation. Figure 8.2 shows the $\cos \theta_{s}^{*}$ distribution for these events and compares it to the $W \rightarrow e \nu$ signal shape. Using the same method used for the $W \rightarrow \tau \nu$ background, it is estimated that $1.7 \%$ of the final event sample is $Z \rightarrow \mathrm{e}^{+} \mathrm{e}^{-}$. 


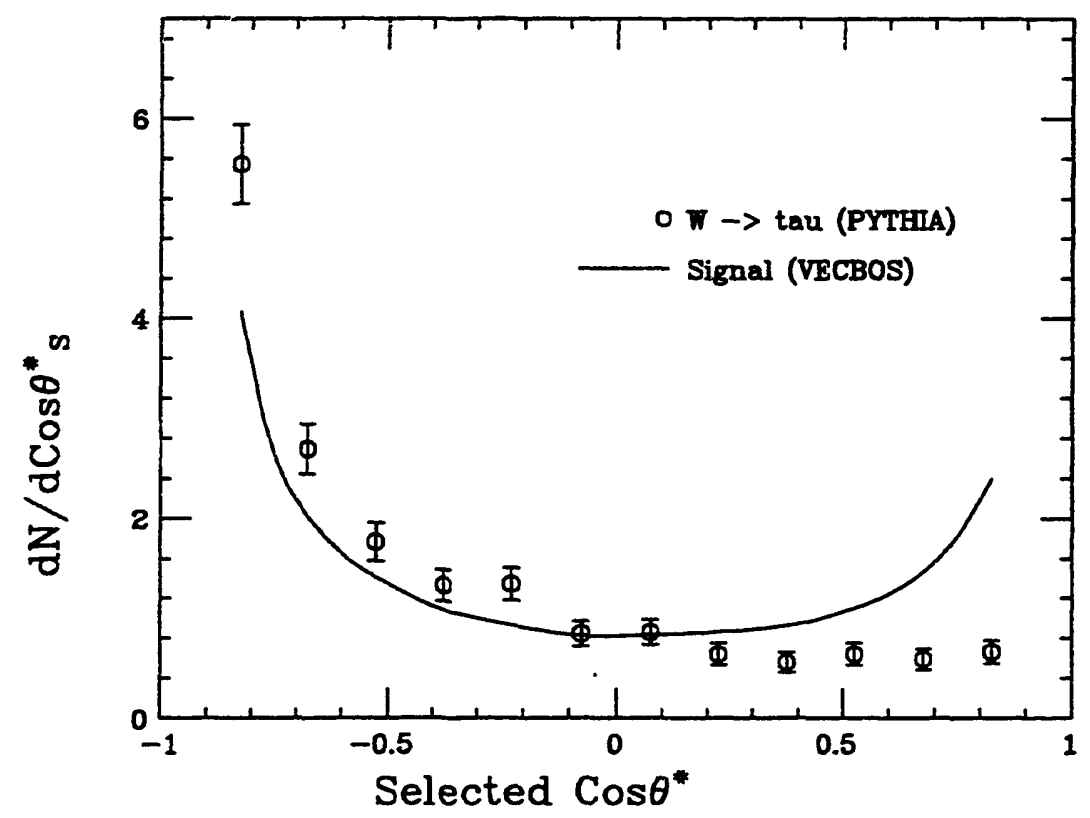

Figure 8.1: The $\cos \theta_{s}^{*}$ distribution for $\mathrm{W}+$ Jet events in which the $\mathrm{W}$ decays to $\tau$ and the $\tau$ to $e \overline{\nu_{e}} \nu_{\tau}$. The line is the VECBOs signal prediction. Both predictions are normalized to 1 between -0.6 and 0.6 . 


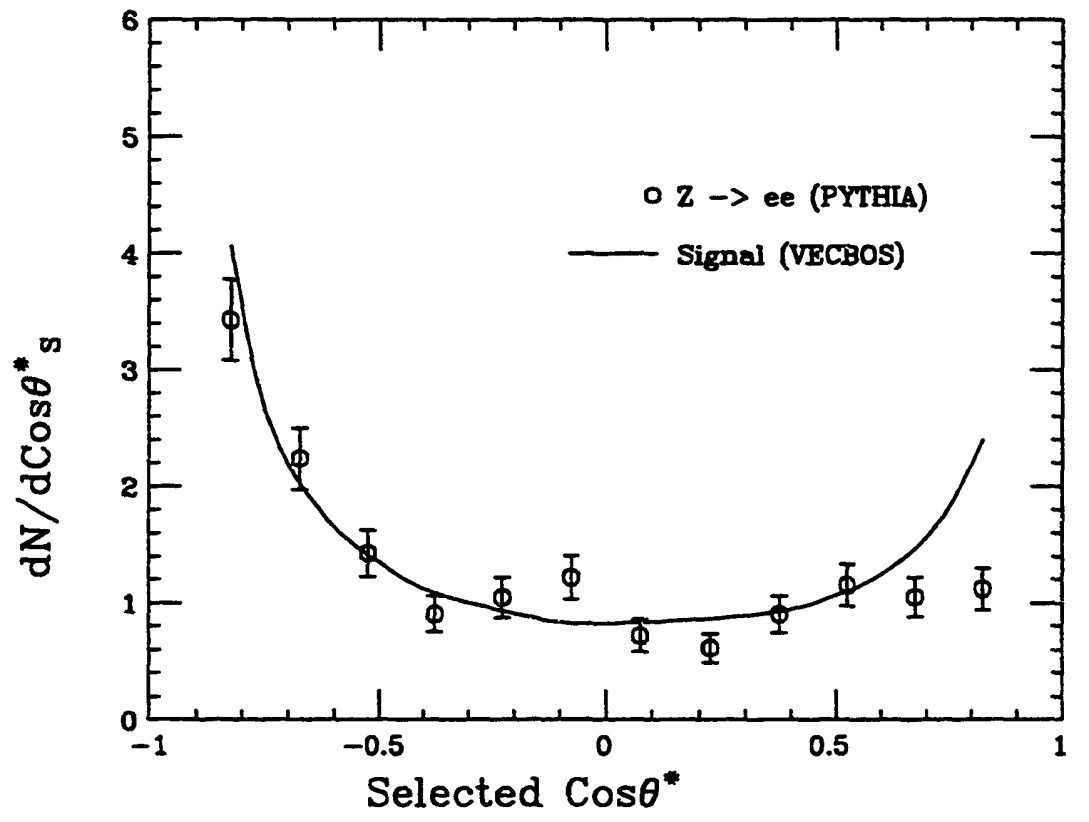

Figure 8.2: The $\cos \theta_{s}^{*}$ distribution for $\mathrm{Z}+$ Jet events in which the $\mathrm{Z}$ decays to electrons. The line is the VECBOS signal prediction. Both predictions are normalized to 1 between -0.6 and 0.6 . 
It is possible that $\mathrm{Z}$ bosons produced without an associated jet represent a background contamination. The mechanism for this would be that one electron is undermeasured but still leaves enough energy in the calorimeter to be identified as a jet (resulting in a $\mathrm{W}+$ Jet signature). This is unlikely, but was non-the-less checked. Pythia was used to generate inclusive $Z \rightarrow \mathrm{e}^{+} \mathrm{e}^{-}$events; that is $Z$ bosons are produced and a leading-log approximation is used to simulate quark and gluon production. After detector simulation, the final $\mathrm{W}+$ Jet cross section resulting from this inclusive sample was actually slightly smaller than the sample in which a jet was always generated. This implies that the leading-log approximation is not generating quite as many jets as it should. It also implies that $\mathrm{Z}$ bosons produced without a jet do not contribute significantly to the $W+$ Jet sample.

\subsection{3 $Z \rightarrow \tau^{+} \tau^{-}$}

$\mathrm{Z}+$ Jet events in which the $\mathrm{Z}$ decays into taus can fake $\mathrm{W}$ events if one of the taus decays to $e \nu \nu$.

This background was estimated by generating $\mathrm{Z}+$ jet events with Pythia in which the $\mathrm{Z}$ was forced to decay to taus. The taus were allowed to decay into all possible channels. The events were then run through the QFL detector simulation. Figure 8.3 shows the $\cos \theta_{s}^{*}$ distribution for these events. Using the same method used for the $W \rightarrow \tau \nu$ and $Z \rightarrow \mathrm{e}^{+} \mathrm{e}^{-}$backgrounds, it is estimated that $0.3 \%$ of the final event sample is $Z \rightarrow \tau^{+} \tau^{-}$.

Inclusive $Z \rightarrow \tau^{+} \tau^{-}$events were also generated using Pythia (the motivation for this was described in the previous section). Again, the final cross section was slightly smaller than the exclusive $Z+$ Jet cross section. This indicates that $\mathrm{Z}$ bosons produced without jets do not contribute significantly to the $\mathrm{W}+$ Jet sample.

\subsection{QCD Background}

It is possible for a QCD jet (i.e. quark or gluon originated) to fragment in such a way that it fakes an electron signature in the detector. One such possibility is a jet that fragments into a charged pion and - neutral pion (the neutral pion's photons provides the EM energy and the charged pion provides the track). Although this is a very rare fragmentation mode for a jet, the cross section for QCD dijets is many orders of magnitude greater than the $W+$ jets cross section.

In order to estimate this background contamination, a rough Monte Carlo simulation of the events was developed. Standard dijet events were generated with the PAPAgeno [20] Monte Carlo. One of the two jets was chosen at random to fragment into a fake electron. Faking the electron was done in two different ways. 


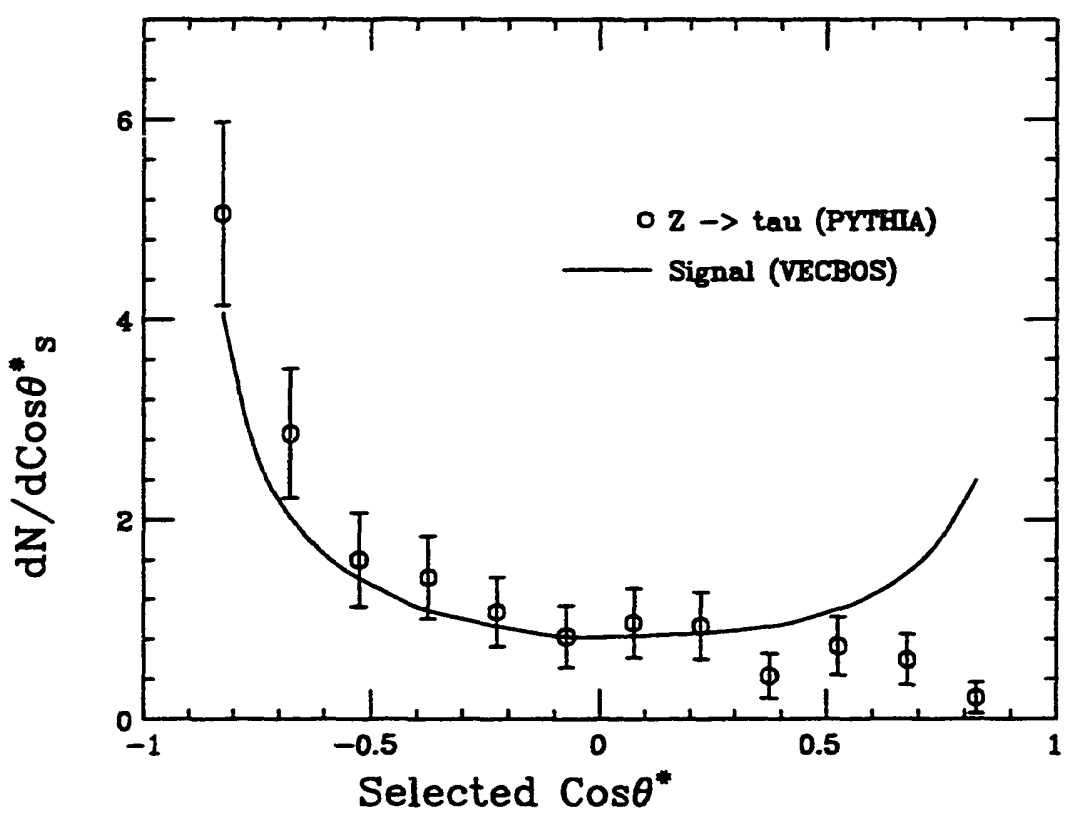

Figure 8.3: The $\cos \theta_{s}^{*}$ distribution for $\mathrm{Z}+$ Jet events in which the $\mathrm{Z}$ decays to taus. The line is the VECBOS signal prediction. Both predictions are normalized to 1 between -0.6 and 0.6 . 
In Method I the identification of the jet was changed to that of an electron (to be fed into the detector simulation) and the momentum of the electron candidate was divided by 2 . The factor of two assumes that the charged pion from the jet passes through the detector without showering, thereby carrying away $1 / 2$ of the jet energy ( $1 / 2$ because the momentum measured from its track must roughly match the energy in the electromagnetic calorimeter in order to pass the electron id cuts). In Method II the electron energy is not divided by two; this represents a jet fragmenting into a single charged pions which then deposits most of its energy in the electromagnetic calorimeter. This method also covers the possibility that the charged particle actually is an electron-perhaps from a photon + jet event in which the photon converted, or from a bottom quark decaying into an electron and a charm quark. In both methods the events are then run through the QDJTMC detector simulation and then through the same analysis as the data.

The number of these events present in the data is estimated by looking at the transverse-mass $\left(M_{t}\right)$ distribution. without the cut at $20 \mathrm{GeV}$. Figure 8.4 shows the $\mathrm{M}_{t}$ distribution of the data compared to the sum of the signal prediction and the other electroweak backgrounds already described. The data shows a slight excess at small values of $\mathrm{M}_{t}$. Fitting the $\mathrm{M}_{t}$ distribution of the QCD Monte Carlo events to this excess predicts that about $3.5 \%$ of the events in this plot are QCD background- $3.5 \%$ is for either Method I or Method II.

The $\mathrm{M}_{t}$ cut of $20 \mathrm{GeV}$ is then applied to the QCD Monte Carlo events. The estimated contamination in the final data sample from the Method I Monte Carlo is $0.01 \%$ (i.e. most of the events were removed by the $M_{t}$ cut). The contamination from the Method II Monte Carlo is estimated to be $1.0 \%$ after the $M_{t}$ cut; figure 8.5 shows the $\cos \theta_{s}^{*}$ distribution for these events. Since the Method I contamination is so small it is not used. The method II background is subtracted from the final $\cos \theta_{s}^{*}$ plot. In Chapter 9 the QCD background will be varied by $\pm 100 \%$ in order to estimate the systematic uncertainty associated with these estimates of the background.

It might seem somewhat unexpected that the $\cos \theta_{s}^{*}$ distribution for the QCD background looks similar to the signal $\cos \theta_{s}^{*}$ distribution; the QCD background events are produced with a much more peaked $\cos \theta^{*}$ distribution. Requiring the electron to be central flattens the $\cos \theta^{*}$ distribution, because this cut is applied directly to the jet that was identified as an electron (in the $W+$ Jet case the central electron cut is not a direct cut on the W). The $\cos \theta^{*}$ distribution is also distorted by making the assumption that the electron and $\mathrm{Z}_{t}$ come from a $\mathrm{W}$ decay (which means that the $\mathrm{P}_{z}^{\nu}$ constraint is completely unrealistic in these events). It is fortunate that the $\cos \theta_{s}^{*}$ distribution for the QCD background is so similar to the signal events; the final $\cos \theta_{s}^{*}$ piot is very insensitive to the predicted number of QCD events in the sample. 


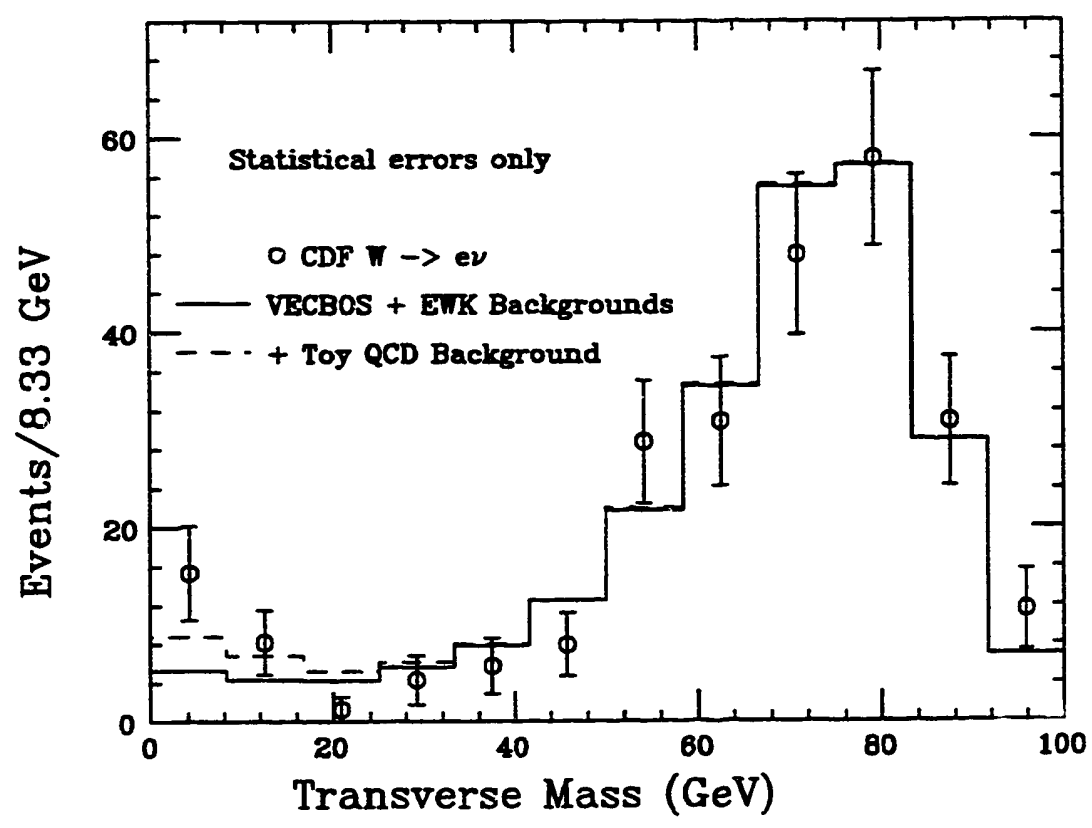

Figure 8.4: The transverse mass distribution for $W+$ Jet events. The points are the CDF data. The solid line is the VECBOs signal prediction with the Pythia electroweak backgrounds added in. The dashed line shows VECBOS+Pythia plus a $3.5 \%$ contribution of the Method II QCD background prediction. 


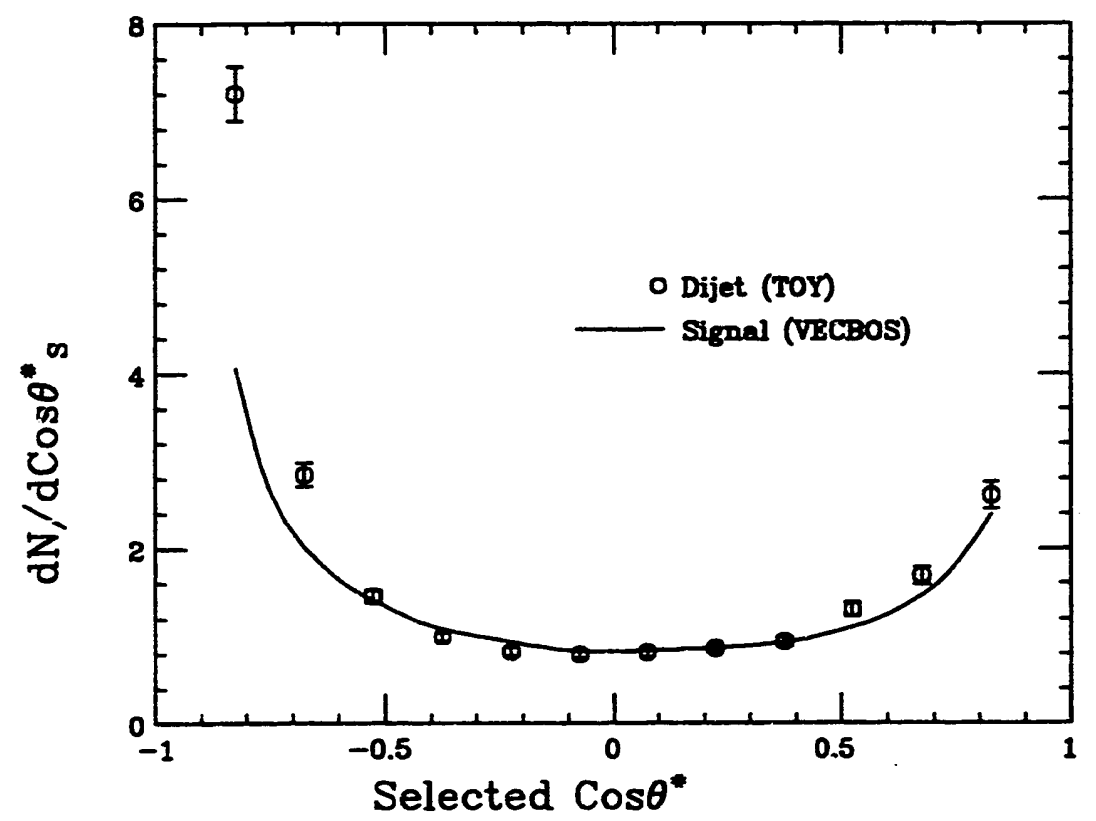

Figure 8.5: The $\cos \theta_{s}^{*}$ distribution for the Method II QCD Monte Carlo. The line is the VECBOS signal prediction. Both predictions are normalized to 1 between -0.6 and 0.6 . 


\subsection{Top Quark Background}

The top quark was discussed in section 1.1.2; the current limit on its mass comes from $\mathrm{CDF}$ and is $113 \mathrm{GeV}[2,3]$. The top quark is predominantly pair-produced and decays into a $\mathrm{W}$ and $\mathrm{a} b$ quark.

Top quark events were generated with the HERwIG[24] Monte Carlo (which was discussed in section 6.2.1) and run through the QFL detector siniulation and the same analysis as the data. Samples were generated using three different top masses: 110,130 , and $150 \mathrm{GeV}$. Figure 8.6 shows the $\cos \theta_{s}^{*}$ distribution for the three different masses. The top quarks decay roughly isotropically, therefore the $\cos \theta=$ distributions are almost flat.

The top quark contribution to the data sample was estimated by comparing the top quark cross section to the total cross section from Pythia for $W+J e t$ signal events and the other electroweak backgrounds. Based on this procedure, a $110 \mathrm{GeV}$ top quark would represent $12 \%$ of the event sample. A 130 (150) $\mathrm{GeV}$ top quark would represent $8.0 \%(2.4 \%)$ of the event sample.

No top quark background is subtracted from the data, because the mass is unknown. The systematic uncertainty is estimated (see section 9.2) by subtracting the $110 \mathrm{GeV}$ top quark. This is actually a large systematic uncertainty on the final result because the top quark $\cos \theta_{s}^{*}$ distribution is so different from the signal events.

\subsection{Summary of Backgrounds}

Table 8.1 summarizes the background estimates. The total estimated background is $6.4 \%$ (not including any top quark). These backgrounds are subtracted from the data before making the final $\cos \theta_{s}^{*}$ plot. Again, uncertainties in the backgrounds will be discussed in Chapter 9. 


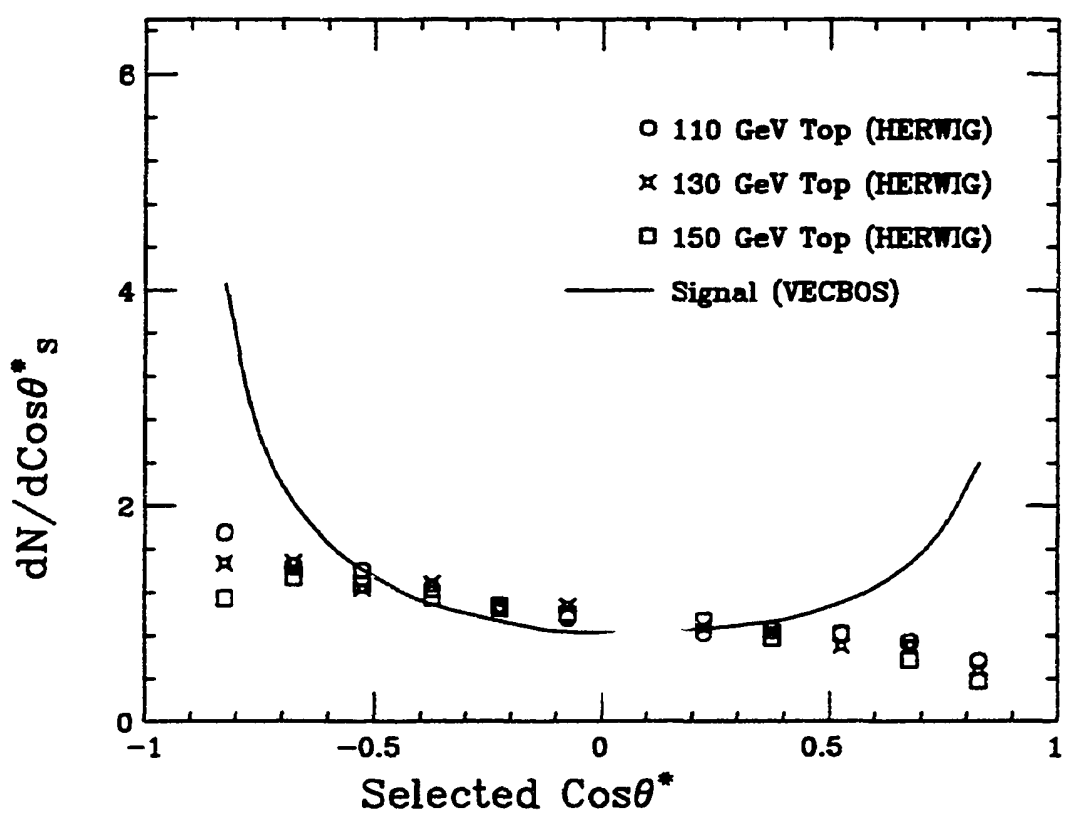

Figure 8.6: The $\cos \theta_{s}^{*}$ distribution for the top quark. The distribution is shown for three different masses: $110 \mathrm{GeV}, 130 \mathrm{GeV}, 150 \mathrm{GeV}$. The line is the vecBos signal prediction. All predictions are normalized to 1 between -0.6 and 0.6 .

\begin{tabular}{||l|r||}
\hline Background & Contamination \\
\hline$W \rightarrow \tau \nu \rightarrow e \nu \nu \nu$ & $3.5 \pm 1.8 \%$ \\
$Z \rightarrow e^{+} e^{-}$ & $1.7 \pm 0.85 \%$ \\
$Z \rightarrow \tau^{+} \tau^{-}$ & $0.3 \pm 0.15 \%$ \\
$\mathrm{QCD}$ & $1.0 \pm 1.0 \%$ \\
$110 \mathrm{GeV}$ Top & $12.0 \pm 6.0 \%$ \\
$130 \mathrm{GeV}$ Top & $8.0 \pm 4.0 \%$ \\
$150 \mathrm{GeV}$ Top & $2.4 \pm 1.2 \%$ \\
\hline
\end{tabular}

Table 8.1: A summary of the backgrounds contaminating the $W+$ Jet event sample. The uncertainties shown are discussed in section 9.2 (as part of the systematic uncertainty on the $\cos \theta_{s}^{*}$ measurement). 


\section{Chapter 9}

\section{Systematic Uncertainties}

There are several sources of systematic uncertainty on the measured $\cos \theta_{s}^{*}$ distribution; however, the uncertainties are all small compared to the statistical errors. This chapter discuses each source of systematic uncertainty and describe how the size of the effect on the $\cos \theta_{s}^{*}$ distribution is estimated.

\subsection{Jet Energy Scales}

The method that CDF used to calibrate the calorimeters' response to jets was described in section 5.3.1. There were two sources of uncertainty in this procedure.

\subsubsection{Absolute Jet Energy Scale}

The absolute calibration of the jet response has been estimated to be uncertain to $\pm 5 \%$ [25]. This is a combination of the uncertainty on the response measured to low energy pions and uncertainties introduced in using a Monte Carlo program to convert the low-energy pion response into a jet response. In order to evaluate the effect of the absolute energy scale on the $\cos \theta_{s}^{*}$ distribution, $\mathrm{W}+$ jet Monte Carlo events were generated and run through the detector simulation with the jet response scaled by $+5 \%$ - and another run with the response scaled by $-5 \%$. Figure 9.1 shows the variations in the $\cos \theta_{s}^{*}$ distribution, for the Monte Carlo events, in response to these scale changes.

The variation around the central value of each bin is used as the estimated systematic uncertainty on that bin. If both variations are on the same side of the central value, then the larger of the two variations is used. In order to indicate the size of the uncertainty: the ratio of the systematic uncertainty on the first (and largest) bin in $\cos \theta_{s}^{*}$ divided by the contents of the bin is $+0 \% /-4.1 \%$-that is, 
both variations lowered this bin (with respect to the normalization region) and the larger effect was $4.1 \%$.

Even though a very large Monte Carlo sample of events was used, the statistical error on the $\cos \theta_{s}^{*}$ bins (in the Monte Carlo sample) is approximately $1.1 \%$. The Monte Carlo predicted that decreasing the jet energy scale by $5 \%$ lowered the largest bin (with respect to the normalization region) by $1.9 \%$ (and increasing the scale by $5 \%$ lowered the bin by $4.1 \%$ ); however, $1.9 \%$ is at the level of the statistical uncertainty of the Monte Carlo events, so it's not clear if this is a real effect or not. The statistical error on the CDF data in the lower bin is $21.6 \%$; therefore, knowing the systematic uncertainties to within $2 \%$ is more than sufficient for this analysis.

\subsubsection{Relative Jet Energy Scale}

The uncertainty on the relative responses of the plug and forward calorimeters, as compared to the central have been estimated to be $\pm 2 \%$ [25]. This comes from the uncertainty in determining the plug and forward responses using the dijet balancing technique described in section 5.3.1-which is largely statistical in origin. In order to evaluate the effect of the relative energy scale on the $\cos \theta_{s}^{*}$ distribution, $\mathrm{W}+$ jet Monte Carlo events were generated and run through the detector simulation with the jet response for the plug and forward calorimeters scaled by $+2 \%$-and another run with the response scaled by $-2 \%$. Figure 9.2 shows the variations in the $\cos \theta_{s}^{*}$ distribution, for the Monte Carlo events, in response to these scale changes. The ratio of the systematic uncertainty on the first (and largest) bin in $\cos \theta_{s}^{*}$ divided by the contents of the bin is $+0 \% /-2.8 \%$.

\subsection{Backgrounds}

In order to estimate the systematic uncertainties introduced by the background subtraction, the number of background events subtracted from the sample was varied.

The electroweak backgrounds $(W \rightarrow \tau \nu, Z \rightarrow e e$, and $Z \rightarrow \tau \tau)$ represent about $5.5 \%$ of the event sample. The number of events in the final sample is 165; therefore, the statistical fluctuations on the electroweak backgrounds will be about $\pm 50 \%$. To estimate the systematic uncertainty due tr, the electroweak background subtraction, the number of background events subtracted was variedindependently for each of the three backgrounds-by $\pm 50 \%$. The uncertainties introduced from these background subtractions are smaller (by more than a factor of 2) than any of the other sources of systematic uncertainty discussed in this chapter. 


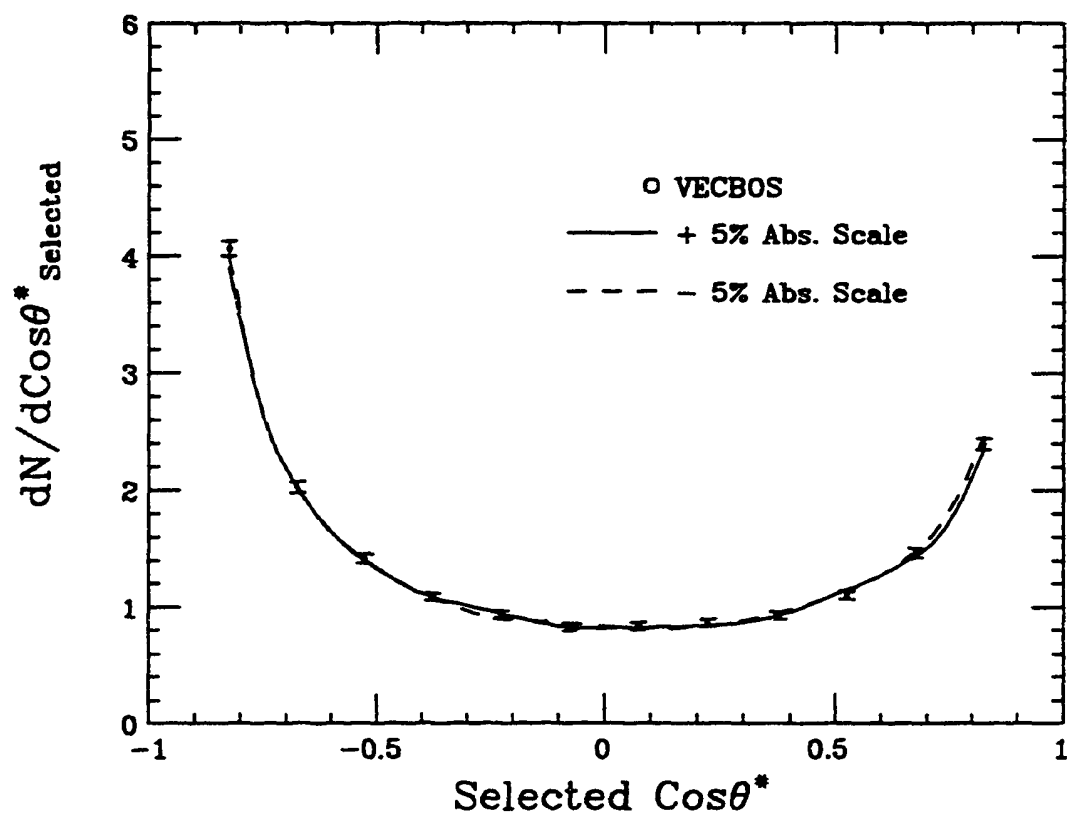

Figure 9.1: Variations in the $\cos \theta_{s}^{*}$ distribution with absolute jet energy scale. The points are the Monte Carlo prediction using the default scale. The solid line is the result of increasing the scale, and the dashed line from lowering the scale. The statistical errors on the curves were omitted, but are similar to the points'. 


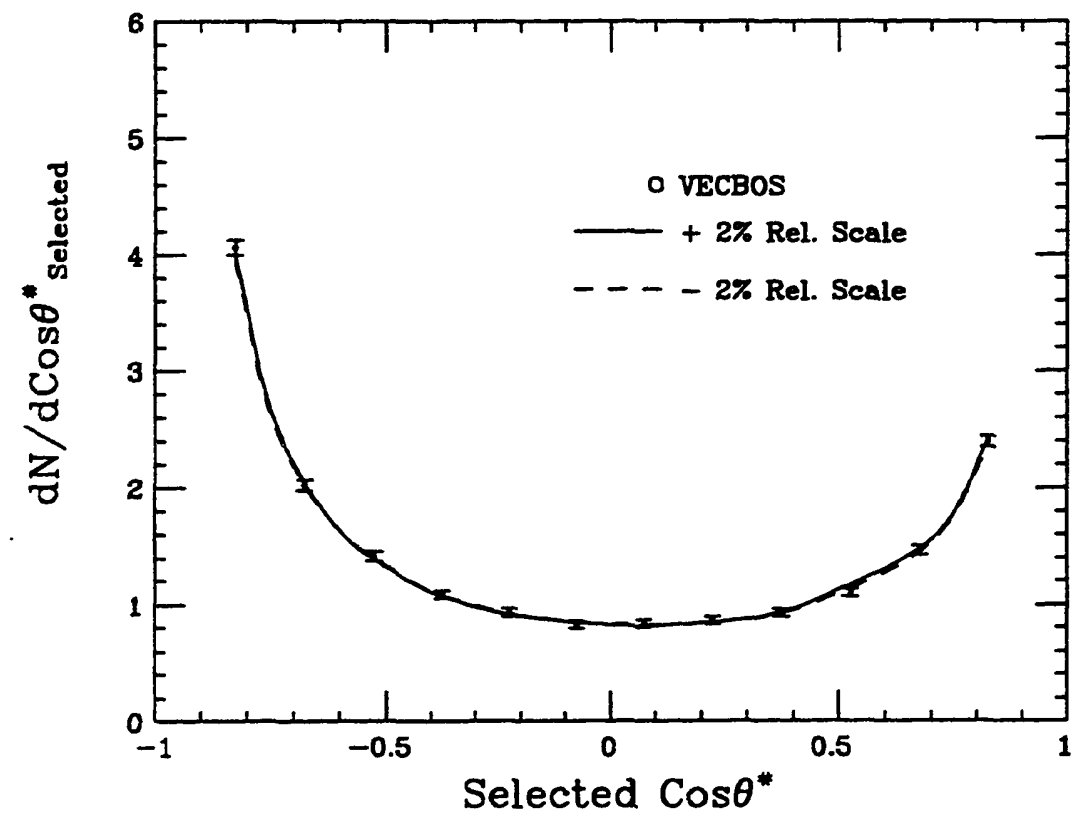

Figure 9.2: Variations in the $\cos \theta_{s}^{*}$ distribution with relative jet energy scale. The points are the Monte Carlo prediction using the default scale. The solid line is the result of increasing the scale, and the dashed line from lowering the scale. The statistical errors on the curves were omitted, but are similar to the points'. 
The background contamination from QCD (dijets, heavy quarks, photon conversions) was estimated to be $1 \%$. In order to be conservative, this background estimate was varied by $\pm 100 \%$.

Figure 9.3 shows the variations in the $\cos \theta_{s}^{*}$ distribution, for the Monte Carlo events, in response to varying the amount of background subtracted. The ratio of the systematic uncertainty on the first (and largest) bin in $\cos \theta_{s}^{*}$ divided by the contents of the bin is $+1.1 \% /-1.2 \%$.

As discussed in section 8.3 , if the top quark's mass is $110 \mathrm{GeV}$ (just above the current $\mathrm{CDF}$ limit) then it would be a $12 \%$ background in the final $\mathrm{W}+$ jet sample. The more massive the top quark is, the less of a background it would be. In order to convert the uncertainty on the top quark's mass into an uncertainty in the $\cos \theta_{s}^{*}$ distribution, the difference in the $\cos \theta_{s}^{*}$ distribution with no top quark background subtracted and with a $110 \mathrm{GeV}$ top quark subtracted was used.

Figure 9.4 compares the $\cos \theta_{s}^{*}$ distribution, for the Monte Carlo events, with and without a $110 \mathrm{GeV}$ top quark background subtracted. The ratio of the systematic uncertainty on the first (and largest) bin in $\cos \theta_{s}^{*}$ divided by the contents of the bin is $+13.2 \% /-0 \%$ (i.e. the subtraction causes the distribution to become more peaked, because the top quark produces a nearly flat $\cos \theta_{s}^{*}$ distribution). This systematic uncertainty is the largest of those described in this chapter.

\subsection{Parton Distribution Functions}

There are uncertainties on the measurements of the parton distribution functions ( $p d f$ 's, see section 2.1 for a discussion of pdf's). The uncertainty on the $\cos \theta_{s}^{*}$ distribution due to the pdf uncertainties was estimated by using four different sets of functions. The default set used in the Monte Carlo generator was HMRSB[19], the other three sets used to estimate the systematic uncertainties were HMRSE[19], KMRSB0-190[26], and MT-B2[27].

In evaluating the effect of the pdf choice on the $\cos \theta_{s}^{*}$ measurement, what needs to be considered is any change in the acceptance as a function of $\cos \theta_{s}^{*}$. The $\cos \theta_{s}^{*}$ distribution produced by the Monte Carlo may change significantly with the pdf choice, but this should not be considered part of the uncertainty on the measurement. Figure 9.5 shows the acceptance, in $\cos \theta_{s}^{*}$, for the four different sets of parton distribution functions. The acceptance is defined to be the ratio of the $\cos \theta_{s}^{*}$ distribution after detector simulation over the $\cos \theta_{s}^{*}$ distribution before detector simulation (the acceptance will be discussed in more detail in section 7.8). Statistical error bars are shown only on the default pdf set, the other curves have similar statistical errors. The differences between the curves are consistent with statistical fluctuations in the Monte Carlo samples; however, the differences will be used as a conservative estimate of the systematic uncertainty. The ratio of 


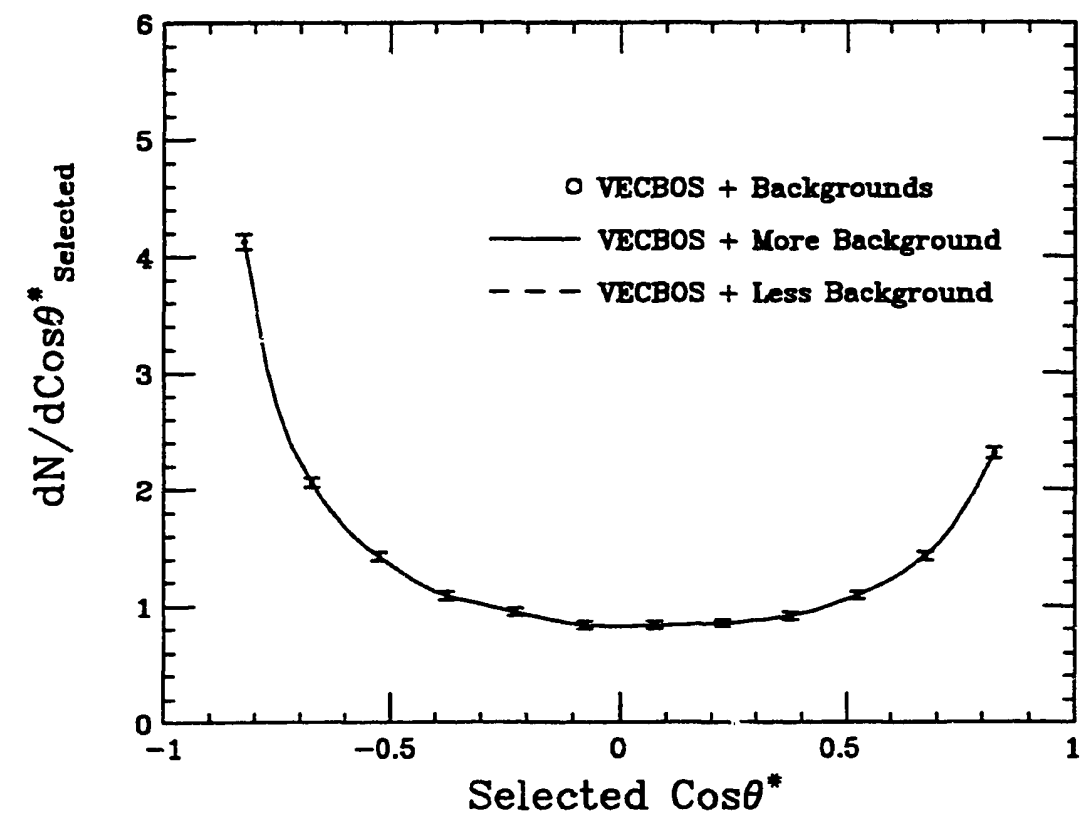

Figure 9.3: Variations in the $\cos \theta_{s}^{*}$ distribution with the amount of background subtracted. The points are the Monte Carlo prediction using the default background. The solid line is the result of subtracting the upper background estimate, and the dashed line of subtracting the lower estimate. The curves lie on top of each other. 


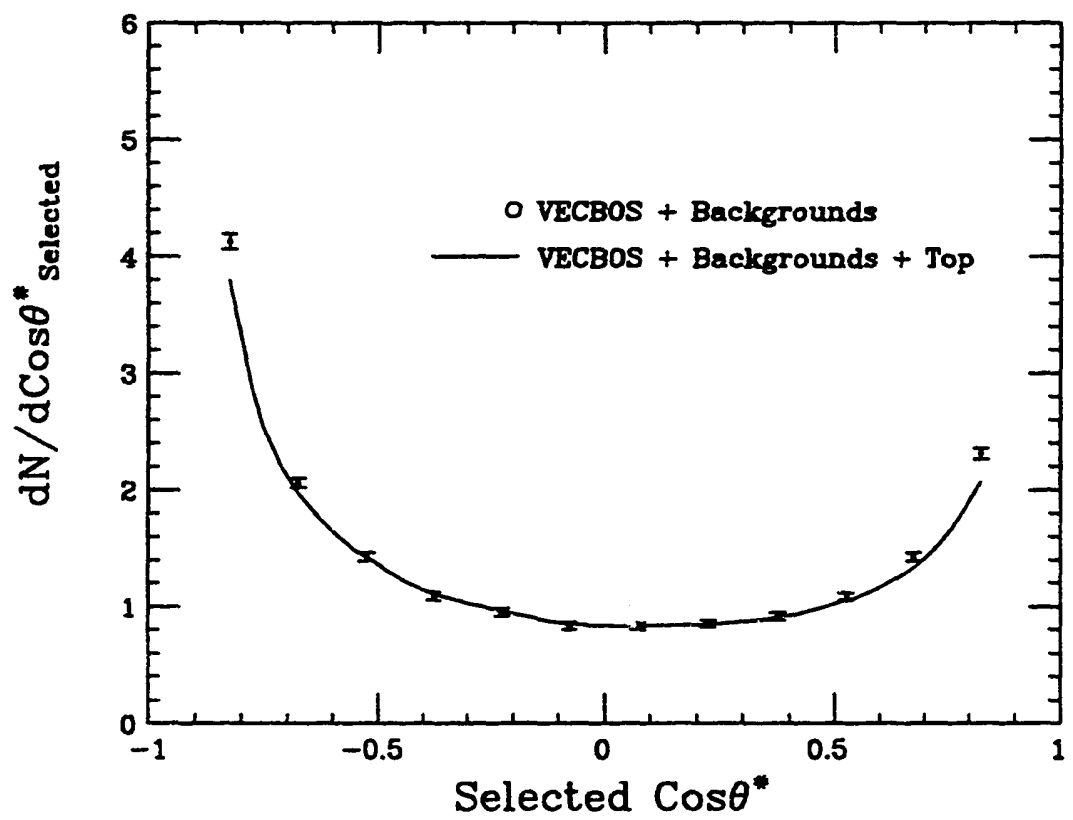

Figure 9.4: Variations in the $\cos \theta_{s}^{*}$ distribution when the top quark background is subtracted. The points are the Monte Carlo prediction using the default background. The solid line is the result of subtracting a $110 \mathrm{GeV}$ top quark signal as well. 
the systematic uncertainty on the first (and largest) bin in $\cos \theta_{s}^{*}$ divided by the contents of the bin is $+2.1 \% /-0.2 \%$.

\section{$9.4 Q^{2}$ Scale}

As discussed in section 2.3.1 there is a theoretical uncertainty in what scale should be used in a tree-level calculation. The default scale used in the Monte Carlo prediction is $1 / 2$ of the $P_{t}$ of the jet (and equivalently $1 / 2$ of the $P_{t}$ of the W). The systematic uncertainty on $\cos \theta_{s}^{*}$ was estimated by using $P_{t}$ and $P_{t} / 4$ for the scale.

As with the parton distribution functions, the scale can effect the initial $\cos \theta_{s}^{*}$ distribution. So, again, the change in $\cos \theta_{s}^{*}$ acceptance was used to estimate the systematic uncertainty. Figure 9.6 shows the acceptance, in $\cos \theta_{s}^{*}$, for the three different choices of scale. The statistical error bars are shown for the default choice of the scale, the other curves have similarly sized errors. The ratio of the systematic uncertainty on the first (and largest) bin in $\cos \theta_{s}^{*}$ divided by the contents of the bin is $+0 \% /-1.2 \%$.

\subsection{W Charge}

If the CDF detector responded differently to positive and negative electrons there would be very little effect in the $\cos \theta_{s}^{*}$ distribution.

The electron's $\eta$ distribution is different for positive and negative $W$ events, and the average $z$-position of the event vertex is displaced about $4.5 \mathrm{~cm}$. Both of these effects may cause a small acceptance difference in the $\cos \theta_{s}^{*}$ distribution for positively and negatively charged W's. However, both effects have been removed by the $\eta$ acceptance correction that was applied in section 5.1 .4 (because the correction was determined using an offset $z$-vertex position and removes known $\eta$ dependencies in the detector's acceptance). The energy corrections applied to the electron also remove any $\eta$-dependent energy response. The $\cos \theta_{s}^{*}$ distribution is very insensitive to any $\eta$ dependent electron efficiencies anyway. Each value of $\cos \theta_{s}^{*}$ is integrated over a large range of electron $\eta$ 's (because the W decay tends to decouple the two quantities).

It might be possible that the detection efficiency is different for position and negative electrons (possibly due to some charge-dependent tracking effect). Since the $\mathrm{W}^{+}$events were inverted, the $\cos \theta_{s}^{*}$ distribution will be insensitive to any differences in detection efficiency for electrons and positrons.

Figure 9.7 shows the $\cos \theta_{s}^{*}$ distribution, as measured using the data, for positive and negative charge events. There is no indication of any charge-dependent 


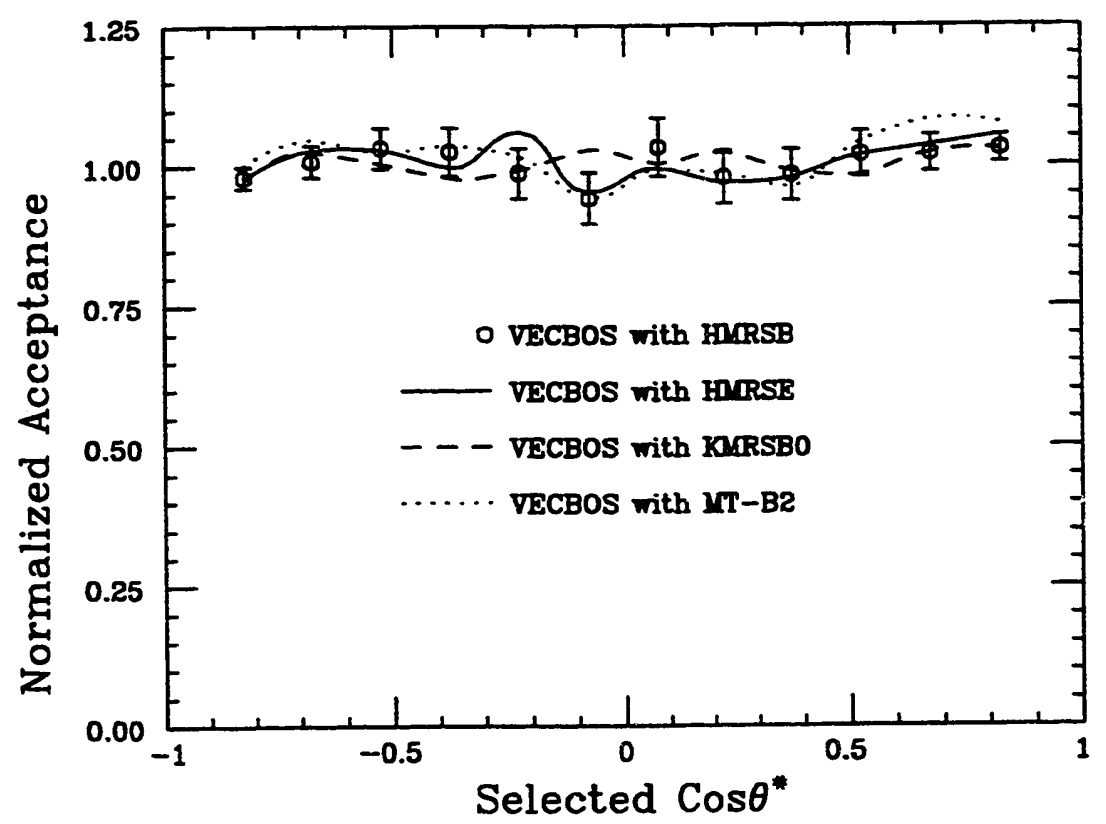

Figure 9.5: Variations in the $\cos \theta_{s}^{*}$ acceptance with the choice of parton distribution functions (pdf). The plot shows the ratio of the detector-simulated $\cos \theta_{s}^{*}$ distribution over the $\cos \theta_{s}^{*}$ distribution before simulation. The points are the default $\mathrm{pdf}$, and the curves show three other choices for the pdf. The statistical errors are shown for the default set only, but the curves have similar errors. The curves (and points) are all normalized to have an average of 1.0 . 


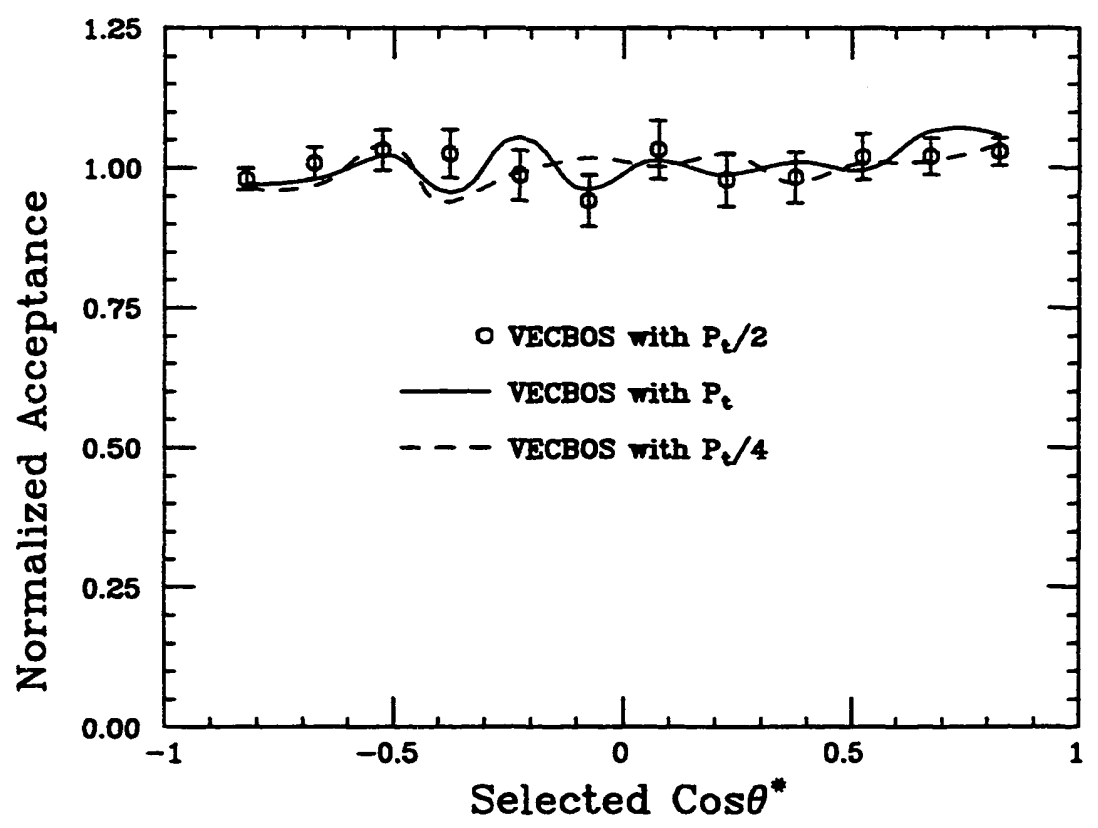

Figure 9.6: Variations in the $\cos \theta_{s}^{*}$ acceptance with the choice of momentum scale $\left(Q^{2}\right)$. The plot shows the ratio of the detector-simulated $\cos \theta_{s}^{*}$ distribution over the $\cos \theta_{s}^{*}$ distribution before simulation. The points are the default scale, and the curves show two other choices for the scale. The statistical errors are shown for the default points only, but the curves have similar errors. The curves (and points) are all normalized to have an average of 1.0 . 


\begin{tabular}{||l|c||}
\hline Effect & $\begin{array}{c}\cos \theta_{s}^{*} \\
\text { Uncertainty }\end{array}$ \\
\hline Statistical & $21.6 \%$ \\
\hline Energy Scale (Abs) & $+0.0 /-4.1 \%$ \\
Energy Scale (Rel) & $+0.0 /-2.8 \%$ \\
PDF & $+2.1 /-0.2 \%$ \\
$Q^{2}$ Scale & $+0.0 /-1.2 \%$ \\
Backgrounds (Not top) & $+1.1 /-1.2 \%$ \\
Top Quark & $+13.2 /-0.0 \%$ \\
\hline Total Systematic & $+13.4 /-5.2 \%$ \\
\hline Total Uncertainty & $+25.4 /-22.2 \%$ \\
\hline
\end{tabular}

Table 9.1: Shown in the table are the uncertainties on the lowest bin $(-0.9$ to -0.75$)$ of $\cos \theta_{s}^{*}$, as a percentage of the contents of the bin. The statistical uncertainty includes the normalization uncertainty (see section 3.5 ).

effect in the $\cos \theta_{s}^{*}$ distribut,ion.

\subsection{Intrinsic $\mathrm{K}_{t}$}

Intrinsic $K_{t}$ was discussed in section 2.3.4. Quickly, it is the small transverse momentum in the $\mathrm{W}+$ jet system caused by the emission of extra low- $\mathrm{P}_{t}$ quarks and gluons. The detector simulation attempts to mimic this effect (see section 6.1.2) by using the $\mathrm{K}_{t}$ distribution as measured from the data. Since the variable $\cos \theta^{*}$ is defined in the $W+$ jet center-of-mass frame, the $\cos \theta_{s}^{*}$ distribution should not be very sensitive to variations in the $K_{t}$. In order to estimate how sensitive $\cos \theta_{s}^{*}$ is to the $K_{t}$, the detector simulation was run without any $K_{t}$ being added to the event. There was no change in the $\cos \theta_{s}^{*}$ distribution, within the statistics available in the Monte Carlo sample, when the $K_{t}$ simulation was turned off.

\subsection{Summary of Systematics}

Each of the systematic uncertainties on the $\cos \theta_{s}^{*}$ distribution are added in quadrature (for each bin) and then added in quadrature with the statistical uncertainty. Table 9.1 summarizes the uncertainties on the $\cos \theta_{s}^{*}$ distribution. The uncertainties shown are for the $\cos \theta_{s}^{*}$ bin covering the range from -0.9 to -0.75 (the largest bin). The systematic uncertainties are all much smaller than the statistical uncertainty, even the top quark mass uncertainty-the largest of the systematic uncertainties. 


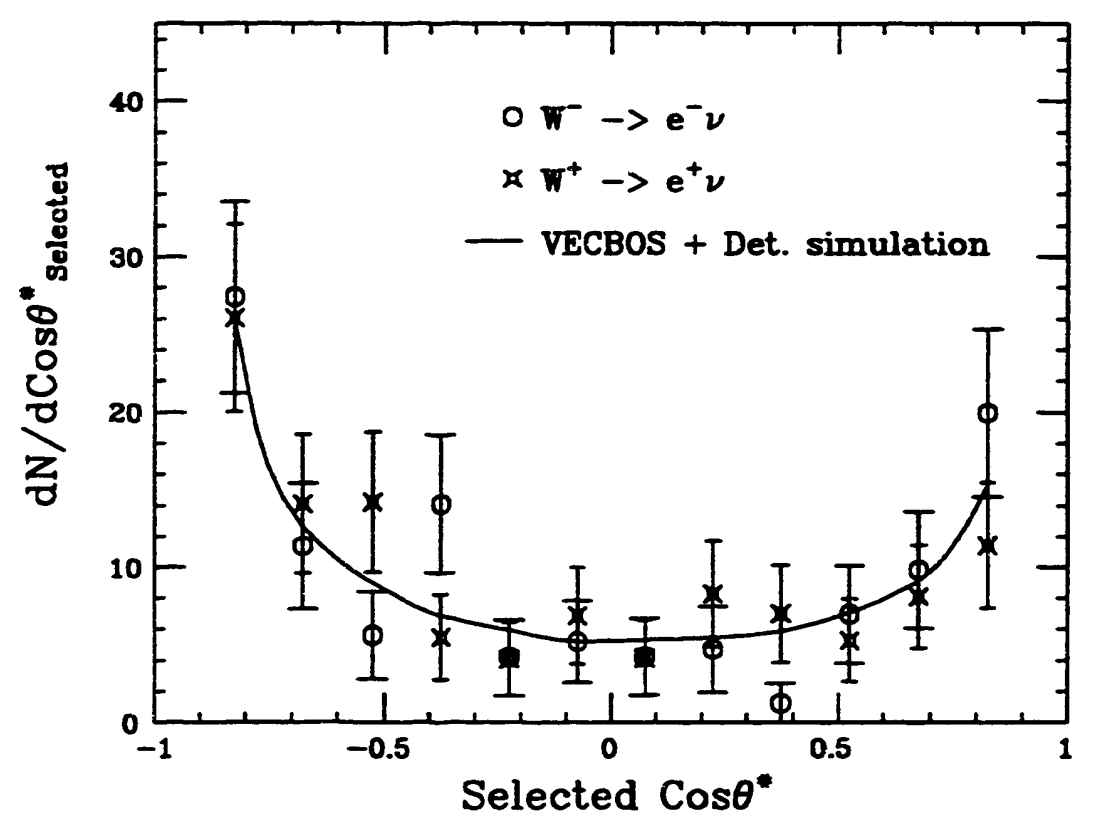

Figure 9.7: The $\cos \theta_{s}^{*}$ distribution shown separately for the $\mathrm{W}^{+}$and $\mathrm{W}^{-}$candidates. The curve is the Monte Carlo prediction normalized to $1 / 2$ the total number of $\mathrm{W}^{+}$ and $W^{-}$events. 


\section{Chapter 10}

\section{Results and Comparison with Theory}

This chapter presents the final $\cos \theta_{s}^{*}$ distribution, and compares it with the leading order (LO) prediction from VECBOs as well as a next-to-leading order (NLO) prediction. Before showing the comparisons, two final issues must be discussed.

\subsection{Methoả for Comparing with Theory}

This section describes the method used to make the $\cos \theta_{s}^{*}$ distribution from a given theory, for direct comparison to the results given here. The theoretical predictions used here are in the form of Monte Carlo programs; the next-to-leading order prediction is available in a program called DYRAD [14]. As was mentioned in section 3.6, this makes it simple to obtain the selected $\cos \theta^{*}$ distribution. It also allows one to make kinematic cuts on the Monte Carlo events in order to match cuts made on the data. This makes the comparisons free of assumptions that may not be satisfied by some theories.

Given a Monte Carlo program that generates events according to some theory, the following steps were used to make a $\cos \theta_{s}^{*}$ distribution for comparison to the data:

1. Make the following lab-frame kinematic cuts:

- Electron $\mathrm{P}_{t}>20 \mathrm{GeV}$, and $|\eta|<0.95$

- Neutrino $\mathrm{P}_{t}>20 \mathrm{GeV}$

- Electron-neutrino Transverse Mass $>20 \mathrm{GeV}$

- Jet $\mathrm{P}_{t}>15 \mathrm{GeV}$ (actualily redundant with the $\mathrm{M}_{s}^{*}$ cut)

- $\Delta R$ (electron - jet $)>0.9$ 
2. Constrain the electron and neutrino to the mass of the $\mathrm{W}$ boson. This gives the two solutions for $\mathrm{P}_{z}^{\nu}$. If the boson mass is not defined for the Monte Carlo, then the constraint was made to the smaller of $80 \mathrm{GeV}$ or the transverse mass (this is the case for DYRAD, see below).

3. Boost, using the Collins-Soper prescription, into the rest frame of the $W$ boson. Identify the selected solution as the one with positive $\cos \alpha_{C S}$ (as described in section 3.2.2).

4. Boost from the lab frame into the $W+$ Jet center-of-mass frame (using the Collins-Soper prescription).

5. Make the following cuts

- $\left|Y_{\text {Boost }}^{s}\right|<1.5$

- $\mathrm{M}_{s}^{*}>121.5 \mathrm{GeV}$

- $\left|\cos \theta_{s}^{*}\right|<0.9$

The DYRAD NLO Monte Carlo program had one other feature that had to be dealt with. Some of the events that it generated had two jets in them. The program merged these two jets if they were separated, in $\eta-\phi$ space, by less than 0.7 (a number chosen to a.pproximate the CDF clustering algorithm). If the jets were not merged, then the event was thrown out if the second jet had $\mathrm{P}_{t}>15$ $\mathrm{GeV}$ and $|\eta|<3.6^{1}$ If the second jet failed these cuts the event was kept, but this second jet was thrown out. The "neutrino" momentum output by DYRAD was the result of adding the electron and the jet. The result of all this is that if the second jet was thrown out, its effect was still seen in the missing- $E_{t}$ (i.e. the Monte Carlo models the $\mathrm{K}_{t}$ kick of the $\mathrm{W}+$ jet system).

None of the theoretical predictions shown in this thesis involve events with multiple jets in them (given that DYRAD only returns events with one jet). If a multi-jet Monte Carlo is to be compared with the data presented here, the following cuts should be applied to the events:

- The $\Delta R$ (electron - jet) $>0.9$ cut should be applied to all jets.

- The jets should be separated from one another by at least 0.7 in $\eta-\phi$ space. This cut only roughly approximates the behavior of the CDF clustering. If the theoretical prediction is sensitive to the specifics of handling the multi-jets, then a comparison with the data presented here may not be arcurate.

\footnotetext{
${ }^{1}$ Events with more than one jet were not removed from the CDF data sample. The DYRAD Monte Carlo program was supplied with this cut built into it; therefore, this cut was used on these Monte Carlo events.
} 


\subsection{Normalization of $\operatorname{Cos} \theta_{\mathrm{s}}^{*}$}

The last issue that must be discussed before the final $\cos \theta_{s}^{*}$ distribution is presented is its normalization. As mentioned in section 3.5 , the $\cos \theta_{s}^{*}$ distribution is normalized so that the average value of the distribution between \pm 0.6 is 1 . Normalizing in this way keeps the final plot from being affected by uncertainties in the various detection efficiencies and the uncertainty on the total integrated luminosity in the sample. Another reason is that the absolute cross section predicted by the theories is sensitive to the parton distribution function, the momentum scale, and the value of the strong coupling constant $\left(\alpha_{s}\right)$; however, the shape of the $\cos \theta_{s}^{*}$ distribution is much less sensitive to these uncertainties.

The statistical errors on the $\cos \theta_{s}^{*}$ must be modified to include the uncertainty on the normalization, because the normalization factor was determined using only 73 events. Call the normalization factor $N$, and the statistical error on $N \sigma_{N}$. If the contents of a $\cos \theta_{s}^{*}$ bin, before normalization, is $A_{i}$, then the normalized bin is $B_{i}=N A_{i}$. If the statistical error on the unnormalized bin is $\sigma_{A i}$, then the error on the normalized bin, including the propagation of the error on $N$, is

$$
\sigma_{B i}=\sqrt{\left(N \cdot \sigma_{A i}\right)^{2}+\left(A_{i} \cdot \sigma_{N}\right)^{2}}
$$

The second term in the radical is due to the propagation of the normalization; therefore, the effect is bigger for the larger bins. For the largest bin in $\cos \theta_{s}^{*}$, the statistical error increases by $24 \%$ (near $\cos \theta_{s}^{*}=0$ it increases by about $6 \%$ ).

\subsection{Measured $\operatorname{Cos} \theta_{\mathrm{s}}^{*}$ Distribution}

Figure 10.1 shows the final selected $\cos \theta^{*}$ distribution. Backgrounds have been subtracted. The inner error bars are the statistical error on each bin. The outer error bars are the combined statistical and systematic uncertainties. The solid curve shows the VECBOS (leading order) prediction. The dashed curve shows the DYRAD (next-to-leading order) prediction. There is very little difference between the leading order and the next-to-leading order predictions.

The agreement between the data and the theoretical predictions is very good. The scattering does indeed seem to be coming from a spin- $\frac{1}{2}$ propagator (the quark propagators in the $\mathrm{W}+$ jet diagrams) as opposed to a spin-1 propagator. Figure 10.2 shows the $\cos \theta_{s}^{*}$ distribution again, this time compared to a prediction in which the propagator has spin-1. This prediction was made by re-weighting the VECBOS events to have the same true $\cos \theta^{*}$ spectrum as dijet events (which have a gluon as the dominant propagator).

As was pointed out in section 3.2.2, the distribution of selected $\left|\cos \theta^{*}\right|\left(\left|\cos \theta_{s}^{*}\right|\right)$ looks very much like the true distribution of $\left|\cos \theta^{*}\right|$. 
Therefore, the $\left|\cos \theta_{s}^{*}\right|$ distribution can be $\operatorname{compared}$ directly to the $\cos \theta^{*}$ distributions measured using other bosons. Figure 10.3 shows the selected $\left|\cos \theta^{*}\right|$ distribution compared to the CDF $\cos \theta^{*}$ measurements for $\operatorname{dijet}[10,11]$ events and photon + jet $[12,13]$ events. The dijet events have a spin-1 (gluon) propagator and show a much more peaked distribution. The photon + jet events have Feynman diagrams very similar to the $\mathrm{W}+$ jet events, and show an angular distribution very similar to the $\mathrm{W}+$ jet events. The NLO theoretical prediction is shown with the $\mathrm{W}+$ Jet events, a NLO prediction is shown with the photon + Jet events, and a $\mathrm{LO}$ prediction with the dijet events. For this plot, the $\mathrm{W}+$ jet distribution was normalized between 0 and 0.3 , because this is the normalization used in the dijet and photon + jet measurements. The dijet events in this plot were taken during the $1987 \mathrm{CDF}$ run, and represent about $7 \mathrm{nb}^{-1}$ of integrated luminosity. The photon events are from the $1992 \mathrm{CDF}$ run, and represent $21 \mathrm{pb}^{-1}$. Again, the $\mathrm{W}+$ jet events are from the 1989 run, and represent $4.05 \mathrm{pb}^{-1}$. 


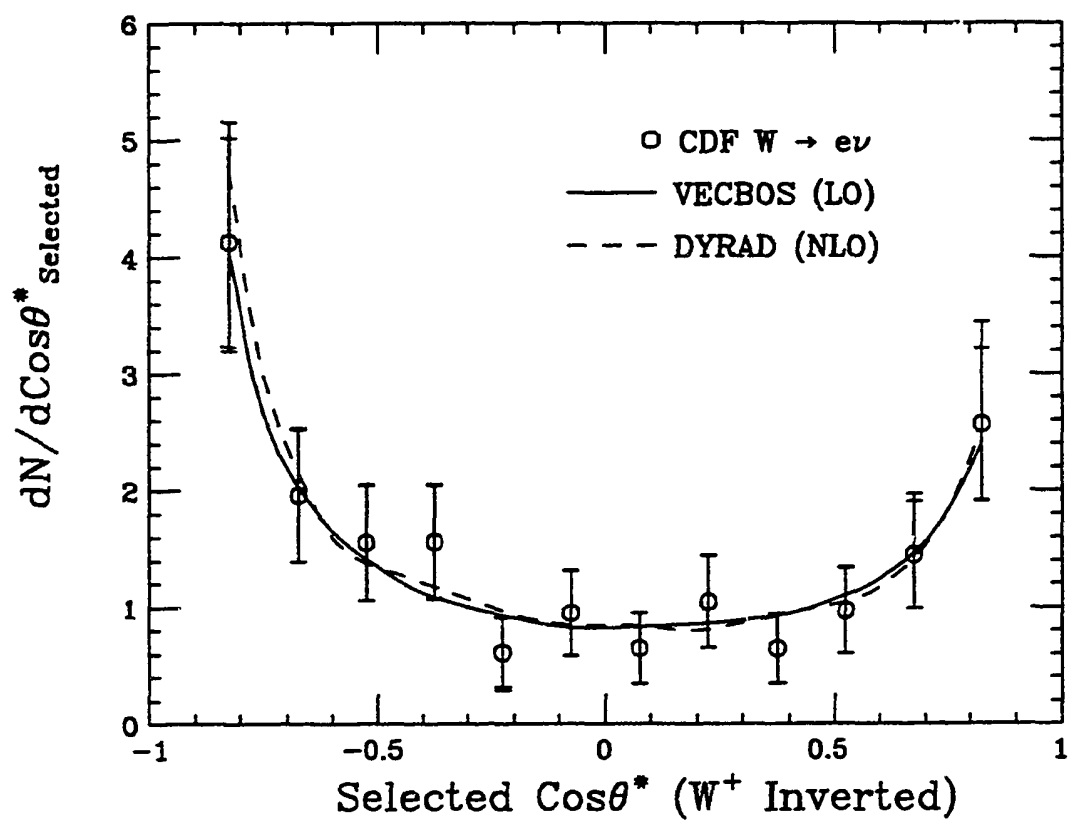

Figure 10.1: The final selected $\cos \theta^{*}$ distribution, including background subtraction. The inner error bars are the statistical error, and the outer bars show the systematic and statistical uncertainties combined (in quadrature). The distribution is normalized to have an average value of 1 between \pm 0.6 . The curves show the leading order and next-to-leading order theoretical predictions. 


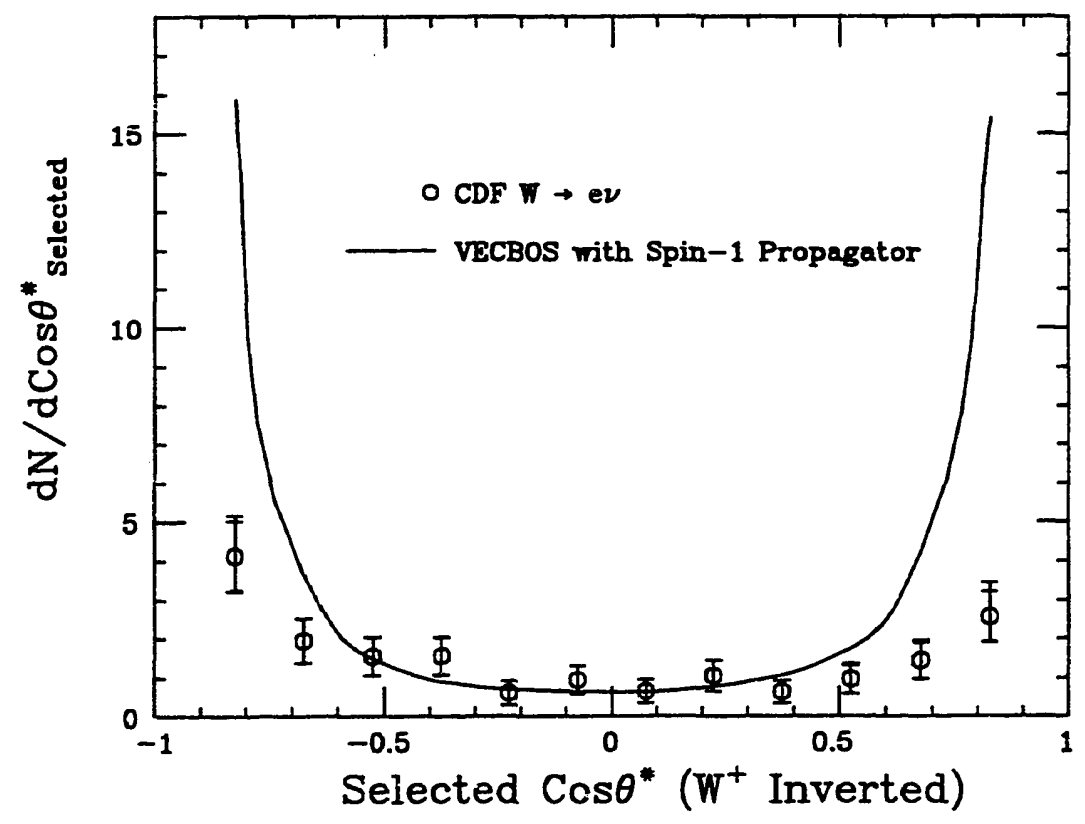

Figure 10.2: The same $\cos \theta_{s}^{*}$ distribution, as shown in figure 10.1, compared to a hypcthetical $\mathrm{W}+$ jet theory with a spin-1 propagator. The spin-1 prediction was made by re-weighting VECBOS events so that the true $\cos \theta^{*}$ distribution was the same as that expected for dijet events. 


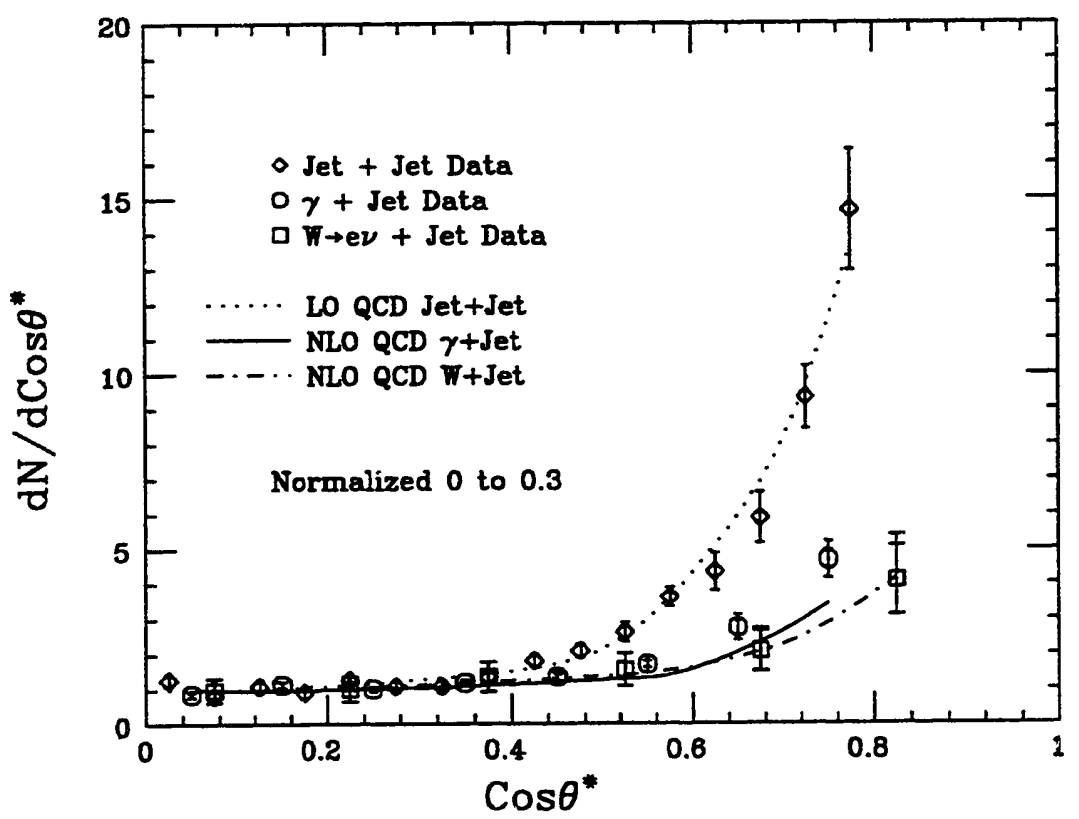

Figure 10.3: The final selected $\left|\cos \theta^{*}\right|$ distribution, including background subtraction. The inner error bars are the statistical error, and the outer bars show the systematic and statistical uncertainties combined. The distribution is normalized to have an average value of 1 between 0 and 0.3 . Also shown are the CDF measurements of $\cos \theta^{*}$ for dijet events and photon + jet events. The curves show the theoretical predictions for each of the three measurements. 


\section{Chapter 11}

\section{Search for Excited Quark States Decaying into $W+$ Jet}

In this chapter attention is turned to the distribution of the energy of the $\mathrm{W}+$ jet system, $M^{*}$. Some current theories[28, 29, 30, 31, 32] predict the existence of new, heavy, particles that would be produced in proton-antiproton collisions and decay into $W+$ jet. These particles would manifest themselves as a resonance in the $W+$ jet energy spectrum.

The model tested in this chapter is excited quark states[28]. In this model the standard up and down quarks are composite states of some smaller particles (usually referred to in the literature as "preons"). The model then supposes that the composite structure of the normal quarks can lead to excited states. In this chapter, a search for excited states of the up $\left(u^{*}\right)$ and down $\left(d^{*}\right)$ quarks (collectively referred to as $q^{*}$ ) is presented.

\subsection{Theory}

The simplest model discussed in the references $[28,29]$ will be considered in this analysis. In this model the $q^{*}$ has spin $\frac{1}{2}$ and weak isospin $\frac{1}{2}$. The lagrangian describing the coupling of the $q^{*}$ state to the Standard Model gauge bosons and a normal quark is restricted, by requiring weak-isospin gauge invariance, to be of the form[28]:

$$
L_{e f f}=\frac{1}{2 \mathrm{M}_{q^{*}}} \overline{Q^{*}} \sigma^{\mu \nu}\left[g_{s} f_{s} \frac{\lambda^{a}}{2} F_{\mu \nu}^{a}+g f \frac{\vec{\tau}}{2} \vec{W}_{\mu \nu}+g^{\prime} f^{\prime} \frac{Y}{2} B_{\mu \nu}\right] q_{L}+\text { h.c. }
$$

In this formula for the lagrangian, $Q^{*}$ represents the isospin doublets of the excited quark, $q_{L}$ represents the lefthanded normal quark. $F^{a}, \vec{W}$, and $B$ are the field strength tensors for the gluon, $S U(2)$, and $U(1)$ gauge fields, and $Y=1 / 3$ is the 


\begin{tabular}{||c|c|c|c||}
\hline Decay Mode & Br. Ratio (\%) & Decay Mode & Br. Ratio (\%) \\
\hline$u^{*} \rightarrow u g$ & 83.4 & $d^{*} \rightarrow d g$ & 83.4 \\
\hline$u^{*} \rightarrow d W$ & 10.9 & $d^{*} \rightarrow u W$ & 10.9 \\
\hline$u^{*} \rightarrow u \gamma$ & 2.2 & $d^{*} \rightarrow d \gamma$ & 0.5 \\
\hline$u^{*} \rightarrow u Z$ & 3.5 & $d^{*} \rightarrow d Z$ & 5.1 \\
\hline
\end{tabular}

Table 11.1: Branching ratios for $1 \mathrm{TeV}$ excited up and excited down quarks. These were determined using $f=1$ and $\alpha_{s}=0.1$.

weak hypercharge. The constants $g_{s}, g$, and $g^{\prime}$ are the Standard Model gauge coupling constants. The parameters $f_{s}, f$, and $f^{\prime}$ are unknown, and depend on the dynamics of the quark compositeness model.

As suggested in the theory references, the three compositeness coupling constants will all be taken as equal, and will be referred to collectively as $f$. The theory references also state that we should expect the coupling, $f$, to be of order 1. Unless otherwise stated, it will be assumed that the compositeness coupling constants all have value 1 . The results of this chapter will be presented in three different ways. Limits on the production cross section for $q^{*}$ states decaying into $\mathrm{W}+$ jet will be shown. These limits are not dependent on the coupling constants. Mass limits for this specific model will also be shown, as well as mass limits as a function of the coupling constant $f$.

It is interesting to look at the branching ratios of the excited quark states; these are shown in table 11.1 for high-mass excited quarks.

\subsection{Previous Searches}

The excited quark model described above is the model generally used when presenting the results of searches for these particles. Aleph[33] has excluded excited quark states below $45 \mathrm{GeV}$. This limit is independent of the model used for the excited quark states, because it only relies on the electroweak charge of the excited quark (because the events searched for were $Z \rightarrow q^{*} \bar{q}^{*}$ ). Aleph[33] has also reported a mass limit for singly produced $q^{*}$ states $\left(Z \rightarrow q^{*} q\right)$ of $88 \mathrm{GeV}$ at $95 \%$ confidence (this limit assumes that the coupling constant, $f$, is equal to one). Finally, a recent limit reported by UA2[34] is a search for excited quark states decaying into two jets. They exclude, at $90 \%$ confidence, the mass range 140 to $288 \mathrm{GeV}$. 


\subsection{Data Samples}

When searching for a new particle, like an excited quark, it is advantageous to have as many events as possible. The data sample used in this search includes the sample used for the $\cos \theta_{s}^{*}$ measurement. The muonic decay mode of the $\mathrm{W}$ will also be included. Data from the 1992 CDF run was also used; these data represent approximately five times as much integrated luminosity as the 1989 data sample.

The following sections describe the data samples used for the excited quark search.

\section{Electron Events from the 1989 Run}

This sample has already been described for the $\cos \theta_{s}^{*}$ measurement. It represents a total integrated luminosity of $4.05 \mathrm{pb}^{-1}$.

\section{Muon Events from the 1989 Run}

This sample was not used in the $\cos \theta_{s}^{*}$ measurement; however, the sample was described (see sections $4.7,5.2$, and 5.6), in parallel with the electron sample, in anticipation of the $q^{*}$ search.

The muon sample, taken during the 1989 run, represents $3.54 \mathrm{pb}^{-1}$.

\section{Electron Events from the 1992 Run}

In the 1992 run, the CDF detector had several upgraded (and additional) components. These upgrades did not significantly alter the components of the detector used for this analysis. Therefore, the same cuts were used to define the 1992 data sample as were used for the 1989 data sample.

The only significant difference in the 1992 run was the electron trigger. The trigger in the 1992 run had a higher threshold on the hardware track that was required to match with the electron. The 1989 trigger required a track with $P_{t}>6$ $\mathrm{GeV}$ (see section 4.7). The 1992 trigger required a track with $\mathrm{P}_{t}>9 \mathrm{GeV}$. The only effect that this has on the analysis is that the trigger efficiency is lower with the higher threshold (efficiencies will be described in section 11.4.1).

The 1992 electron sample represents a total integrated luminosity of $21.3 \mathrm{pb}^{-1}$.

\section{Muon Events from the 1992 Run}

In the 1992 run, extended muon coverage was added to the central muon detectors. Work is still in progress to understand the behavior of the muon extension; it was 
not used for this analysis. Therefore, the muon detectors are also the same as those used in the 1989 run. The efficiency of the 1992 muon trigger was also slightly lower than the efficiency for the 1989 run, which will be discussed in section 11.4.1.

The 1992 muon sample represents a total integrated luminosity of $21.3 \mathrm{pb}^{-1}$.

\subsection{QSTAR Monte Carlo}

In order to predict distributions for the $q^{*}$ events, a Monte Carlo program was developed. The program, referred to from now on as QSTAR, is a leading order parton level Monte Carlo based on the matrix elements in [28]. QSTAR produces $q^{*}$ states from quark-gluon interactions, the $q^{*}$ is then decayed isotropically (as is expected for a spin- $\frac{1}{2} q^{*}$ ) into a $W$ and a quark. The $W$ is also decayed isotropically (that is, the $\mathrm{W}$ is not polarized in any particular direction).

The matrix elements in QSTAR are leading order, and so the parton distribution functions used are CTEQ1L[5], which is a recent leading order set. The momentum scale, at which the parton distribution functions and the strong coupling constant $\left(\alpha_{s}\right)$ are evaluated, was chosen to be the mass of the $q^{*}$ generated in each event.

The output of the QSTAR Monte Carlo was then input to the QDJTMC detector simulation (QDJTMC was described in section 6.1.2). The QSTAR events were then run through exactly the same analysis procedure as the data.

\subsubsection{Efficiencies}

In order to determine a limit, the Monte Carlo needs to be properly normalized to the data. The Monte Carlo events are weighted to match the integrated luminosity in each event sample. They are also weighted to account for efficiencies that are not included in the QDJTMC detector simulation. These efficiencies are described in the following sections.

\subsubsection{Electron Efficiencies}

The combined efficiency of the electron identification cuts and the trigger requirements, for the 1989 data sample, is 0.81 [35]. Table 11.2 shows a breakdown of the efficiency into each electron ID cut. These effects were not accounted for in the QDJTMC detector simulation; therefore the QSTAR and VECBOS events were weighted by 0.81 .

For the 1992 electrons, the same efficiencies were used except that the trigger efficiency was determined to be $4.3 \%$ less efficient than the 1989 trigger. For 


\begin{tabular}{||l|c||}
\hline Selection Requirement & Efficiency (\%) \\
\hline Had/EM Cut & $99 \pm 1$ \\
E/P Cut & $93 \pm 1$ \\
Track-CES Matching in x & $97 \pm 1$ \\
Track-CEM Matching in z & $98 \pm 1$ \\
LSHR Cut & $97 \pm 1$ \\
Isolation Cut & $96 \pm 1$ \\
CES $\chi^{2}$ & $97 \pm 1$ \\
Photon conversion Removal & $96.5 \pm 1.5$ \\
Electron Trigger (89) & $97.3 \pm 0.5$ \\
Electron Trigger (92) & $93.1 \pm 0.5$ \\
\hline \hline Combined Efficiency (89) & $81 \pm 4$ \\
\hline \hline Combined Efficiency (92) & $77 \pm 4$ \\
\hline
\end{tabular}

Table 11.2: A breakdown of the efficiencies in the electron sample[16, 35] that are not included in the Monte Carlo simulation. The efficiencies for the electron ID cuts were determined with all other ID cuts applied (correlations are included in the combined efficiency). The trigger efficiencies are shown for both the 1989 and the 1992 CDF runs, and the combined efficiency is shown for both runs. 


\begin{tabular}{||l|l||}
\hline Selection Requirement & Efficiency (\%) \\
\hline Track-CMU Matching & $96.0 \pm 1.0$ \\
Tower Energy Cut & $98.7_{-0.4}^{+0.3}$ \\
Isolation Cut & $98 \pm 1$ \\
Track Finding Eff. & $98.7 \pm 1.0$ \\
CMU Stub Finding Eff. & $98.6_{-3.3}^{+1.2}$ \\
Cosmic Ray Removal & $99.7 \pm 0.3$ \\
Muon Trigger (89) & $91 \pm 2$ \\
Muon Trigger (92) & $87.3_{-1.9}^{+1.5}$ \\
\hline \hline Combined Efficiency (89) & $82 \pm 4$ \\
\hline \hline Combined Efficiency (92) & $79 \pm 4$ \\
\hline
\end{tabular}

Table 11.3: A breakdown of the efficiencies in the muon sample[36] that are not included in the Monte Carlo simulation. The efficiencies for the muon ID cuts were determined with all other ID cuts applied (correlations are included in the combined efficiency). The trigger efficiencies are shown for both the 1989 and the 1992 CDF runs, and the combined efficiency is shown for both runs.

comparison to the 1992 electron sample, the VECBOS and QSTAR events were weighted by 0.77 .

\subsubsection{Muon Efficiencies}

The combined efficiency of the muon identification cuts and the trigger requirements, for the 1989 data sample, is $0.82[36]$. Table 11.3 shows a breakdown of the efficiency into each muon ID cut. These effects were not accounted for in the QDJTMC detector simulation; therefore the QSTAR and VECBOS events were weighted by 0.82 .

For the 1992 muons the same efficiencies were used except that the trigger efficiency was determined to be $4.1 \%$ less efficient than the 1989 trigger. For comparison to the 1992 muon sample, the VECBOS and QSTAR events were weighted by 0.79 .

\subsubsection{Mass-dependent Efficiencies}

Of the extra efficiencies applied to the Monte Carlo events, only one is expected to have any significant dependence on the $W+$ jet mass, $M^{*}$ : the $\mathrm{Had} / \mathrm{EM}$ cut on the electron (described in section 5.1.2). As the electron energy increases, the efficiency of the Haà/EM cut decreases due to leakage of the electron shower out the back of the electromagnetic calorimeter and into the hadronic calorimeter. Much of this energy dependence is removed by the sliding Had/EM cut, but the 
sliding cut hits the trigger's Had/EM cut (which is $12.5 \%$ ) when the electron has an energy of $150 \mathrm{GeV}$. Figure 11.1 shows the loss in efficiency of the Had/EM cut as a function of electron energy. The QSTAR and VECBOS Monte Carlo events were weighted to include this effect (at high masses this lowers the Monte Carlo predictions by about $4 \%$, which has only a small effect on the excited quark limits.).

\subsection{Analysis}

This section describes the analysis method used for determining the limits on excited quark states. The goal is to compare the distribution for the $\mathrm{W}+$ jet energy $\left(M^{*}\right)$ to the VECBOS Monte Carlo prediction and set limits on the amount of $q^{*}$ signal (using the QSTAR Monte Carlo) that can be present in the data. The method used for making the $M_{s}^{*}$ distribution is very similar to that used for making the $\cos \theta_{s}^{*}$ distribution.

The following subsections describe the differences between the analysis method used to measure the selected $\cos \theta^{*}$ distribution and the method used to measure the selected $M^{*}$ distribution. Later sections will then describe exactly how the cross section (and mass) limits are obtained from the $\mathbf{M}_{s}^{*}$ distribution.

\subsubsection{Differences from the $\operatorname{Cos} \theta_{s}^{*}$ Analysis}

The following is a list of the differences between the $\cos \theta_{s}^{*}$ and the $M_{s}^{*}$ analyses:

- The correction for the electron $\eta$ acceptance, described in section 5.1.4, is not applied to the events. Weighting the events to correct for this acceptance makes the calculation of cross section limits much more difficult, because it produces bins in the $\mathrm{M}_{s}^{*}$ histogram with non-integer numbers of events. The electron acceptance is modeled in the VECBOS and QSTAR Monte Carlos; therefore, the data and the Monte Carlo predictions can be compared without the $\eta$ corrections.

- The $20 \mathrm{GeV}$ transverse mass cut (described in section 5.5) is not applied in the $q^{*}$ analysis. This cut was used in the $\cos \theta_{s}^{*}$ analysis because it removed background events and retained most of the signal events. However, the transverse mass cut removes almost $15 \%$ of the QSTAR signal events, because the $\mathrm{W}$ boson from the $q^{*}$ decay typically has a very large transverse momentum. The background events that enter the sample have an $\mathrm{M}_{s}^{*}$ distribution that is similar to the $\mathrm{W}+$ jet events; therefore, these events were allowed into the sampie in exchange for preserving the $q^{*}$ events (backgrounds will be discussed in section 11.7.3). 


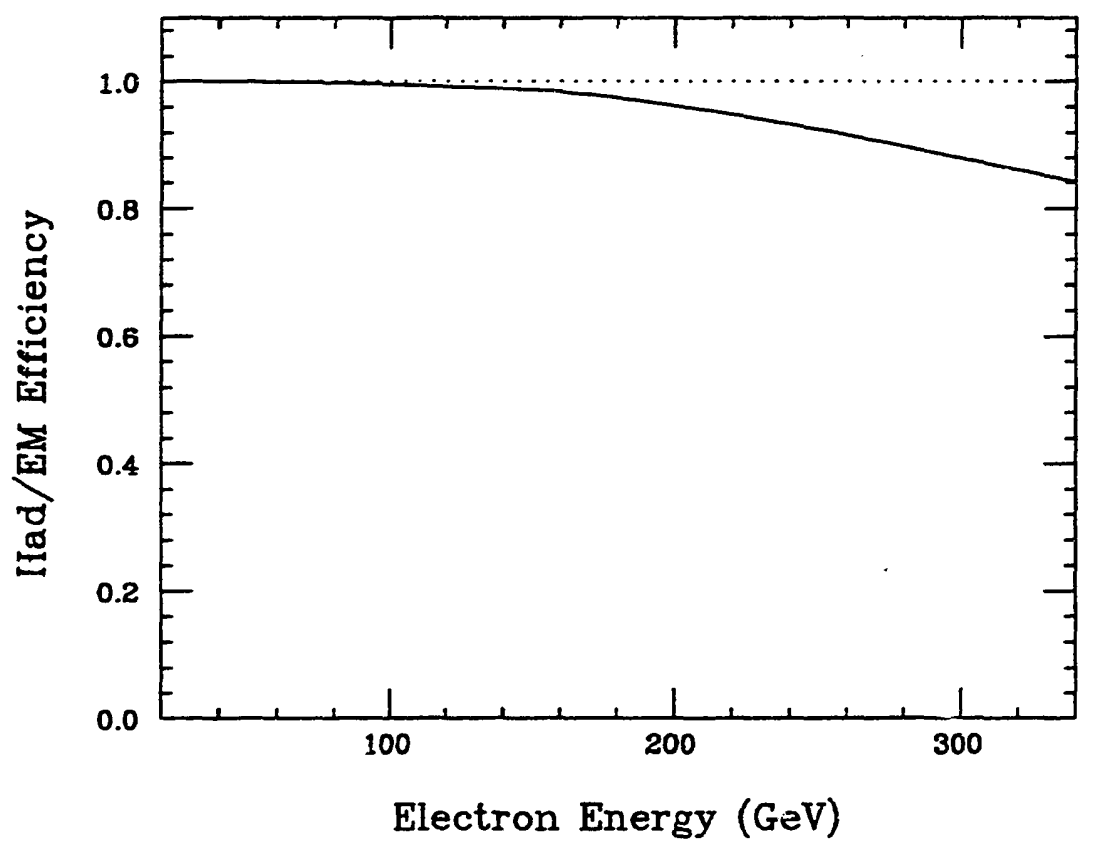

Figure 11.1: The energy dependent efficiency of the Had/EM cut for electrons. Electrons with more than $340 \mathrm{GeV}$ of energy are exciuded from the event sample. 
- The muon ID cuts described in section 5.6 remove most of the cosmic ray events in the data; however, a small number of these events seem to remain. These events tend to have the following characteristics: the jet back-to-back, in $\phi$, with the muon, and the neutrino in the same direction, in $\phi$, as the jet. This indicates that the missing- $E_{t}$ in the event is probably due to the jet being undermeasured (the jet is probably the result of the muon showering in the calorimeter). Figure 11.2 shows the difference in $\phi$ between the jet and the missing- $E_{t}\left(\Delta \phi_{\nu J}\right)$. The figure also shows the prediction from the VECBOS-QDJTMC Monte Carlo. The plot shows that there is an excess of events with $\Delta \phi_{\nu J}<0.4$.

These excess events tend to have very high $\mathrm{W}+$ jet mass and so a better limit can be achieved by removing them. A cut is made, for both the electron and the muon events, requiring $\Delta \phi_{\nu J}<0.4$. This cut may also remove some of the dijet background in the sample, which is why it is also applied to the electron everits. The $\Delta \phi_{\nu J}$ cut removes about $6 \%$ of the $q^{*}$ signal events for a $150 \mathrm{GeV} q^{*}$, and less than $1 \%$ for a $600 \mathrm{GeV} q^{*}$.

- As has already been mentioned, the electron trigger requires a Had/EM cut of $12.5 \%$. For electrons with energy greater than $340 \mathrm{GeV}$ the trigger $\mathrm{ADC}$ 's in the central electromagnetic calorimeter can saturate, causing a loss in trigger efficiency. Rather than try to simulate this loss in efficiency, a cut is made requiring the electron energy to be less than $340 \mathrm{GeV}$.

It might seem wasteful to make such a cut when doing a search for massive objects; however, the Monte Carlo predicts that, even for a $650 \mathrm{GeV} \mathrm{q}$, the expected loss in the signal events is less than $2 \%$. In order to be consistent between the samples, the muons are also required to have an energy of less than $340 \mathrm{GeV}$. The tracking resolution is getting quite large $(37 \%)$ for muons at this energy, so it is not unreasonable to apply this cut to the muons as well. No events were lost from the data sample because of this cut.

\subsubsection{Definition of "Selected" Solution for the $q^{*}$ Search}

For the measurement of $\cos \theta_{s}^{*}$, the "selected" solution was chosen so that it would be the "true" solution as often as possible. For making the selected $\mathrm{M}^{*}$ distribution for the excited quark search, a different method was used to choose one of the solutions. The "selected" solution was defined to be the solution with the smaller value of $\mathrm{M}^{*}$.

This definition was used for two reasons. As part of this analysis, the selected $\mathrm{M}^{*}$ distribution must be presented. The most conservative way to present this plot is by using the smaller mass solution. In this way the distribution doesn't have many high mass events that only appear at high mass because the wrong 


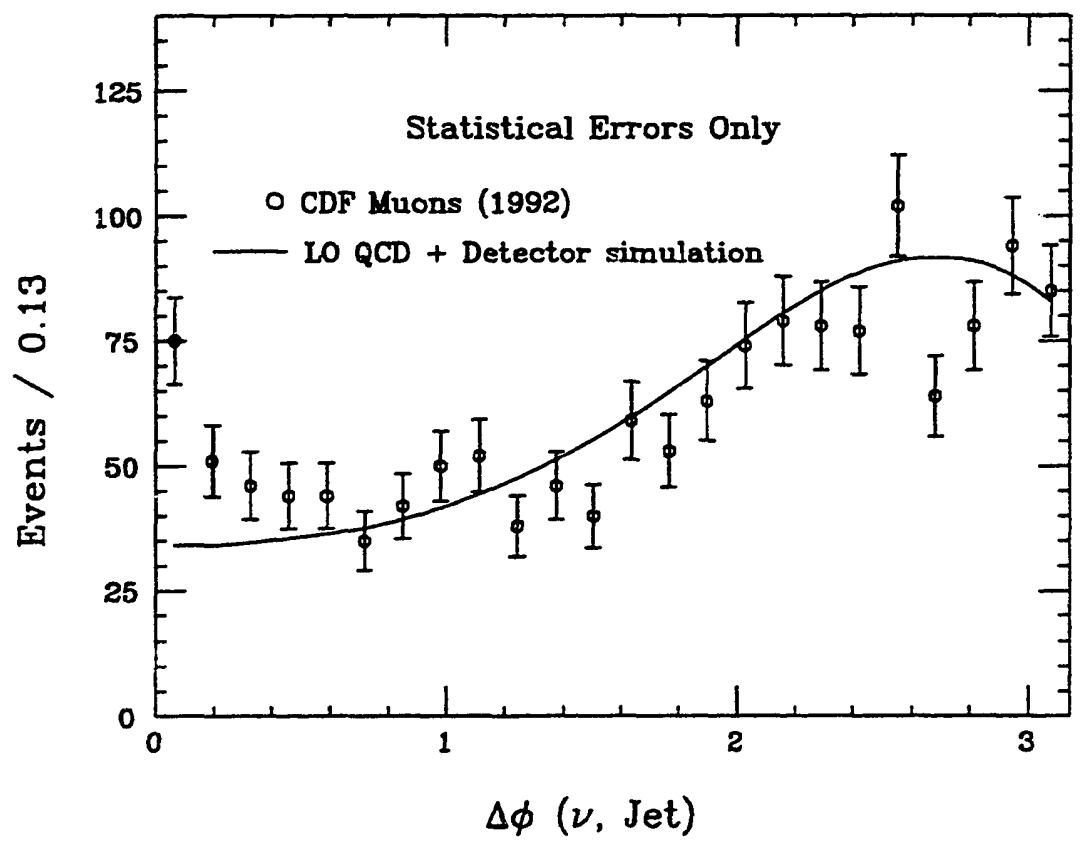

Figure 11.2: The difference in $\phi$ between the missing $E_{t}$ and the leading jet. The points are the 1992 muon data and the curve is a smoothed VECBOS prediction normalized to have the same number of events as the data. 
event solution was chosen. This is essentially a decision based on aesthetics. Monte Carlo studies, which will be described in detail in section 11.8.1, predict that using this definition of selected actually results in better excited quark limits than using the $\cos \theta^{*}$ definition.

\subsubsection{Distributions of Selected $M^{*}$}

Figure 11.3 shows the selected $\mathrm{M}^{*}$ distribution compared to the VECBOS-QDJTMC prediction (normalized to have the same number of events as the data). The plot also shows the predicted QSTAR signal for three different $q^{*}$ masses (with an absolute normalization). In this plot, the bins start at the $\mathrm{M}_{s}^{*}$ cut of $121.5 \mathrm{GeV}$, and each consecutive bin has a width equal to the mass resolution ${ }^{1}$. The points were plotted at the average mass value in the bin. This plot shows that the Monte Carlo prediction agrees very well with the data, and that there is no significant evidence for excited quark states.

Figure 11.4 shows the $M_{s}^{*}$ distribution for each of the data samples separately. In these plots, the VECBOS-QDJTMC predictions were normalized using the same factor used to normalize figure 11.3.

Figure 11.5 shows the calorimeter display for the highest-mass 1992 electron event. This event has a mass of $626 \mathrm{GeV}$ (the other mass solution is $641 \mathrm{GeV}$ ). Figure 11.6 shows the calorimeter display for the other high-mass 1992 electron event. This event has a mass of $574 \mathrm{GeV}$ (the other mass solution is $600 \mathrm{GeV}$ ).

When calculating the cross section (or mass) limits on excited quark states, the $\mathrm{M}_{s}^{*}$ distribution is binned differently than was shown in figure 11.3. The events are binned starting at $121.5 \mathrm{GeV}$ with bins of width $25 \mathrm{GeV}$.

\subsection{Determination of the Excited Quark Lim- its}

Using the $\mathrm{M}_{s}^{*}$ distributions, limits can be set on the amount of the excited quark events that can be present in the data. A $95 \%$ confidence limit on the cross section for producing $q^{*}$, times the branching ratio into $W+$ Jet, was determined using a binned maximum likelihood method.

The likelihood function, for a $q^{*}$ of mass $\mathrm{M}_{q^{*}}$, was determined by first forming an $\mathrm{M}_{s}^{*}$ prediction which was a combination of the VECBOS prediction and the

\footnotetext{
${ }^{1}$ The mass resolution was determined by fitting each $q^{*}$ signal, after detector simulation, with a gaussian. The width of the gaussian was then taken as the mass resolution, after subtracting the contribution from the natural width of the $q^{*}$ state $\left(\sigma_{q^{*}} \approx 0.04 \mathrm{M}_{q^{*}}\right)$. The mass resolution is approximately $\sigma_{M}=0.13 M$.
} 


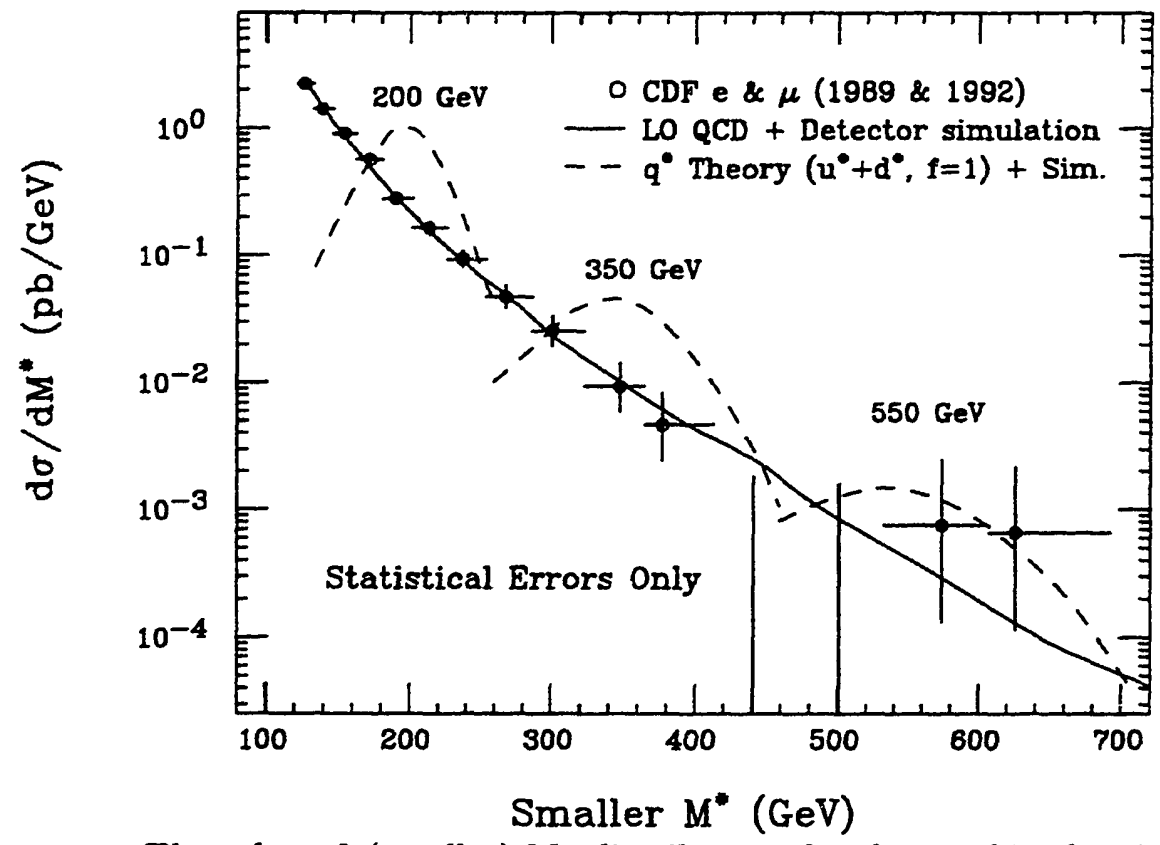

Figure 11.3: The selected (smaller) $M^{*}$ distribution for the combined 1989 and 1992, electron and muon samples. The solid curve is the VECBOS-QDJTMC prediction, normalized to the number of events in the data. The dashed curves show $q^{*}$ signal predictions, absolutely normalized. The bins have been chosen to be equal to the mass resolution. 


\section{Statistical Errors Only}
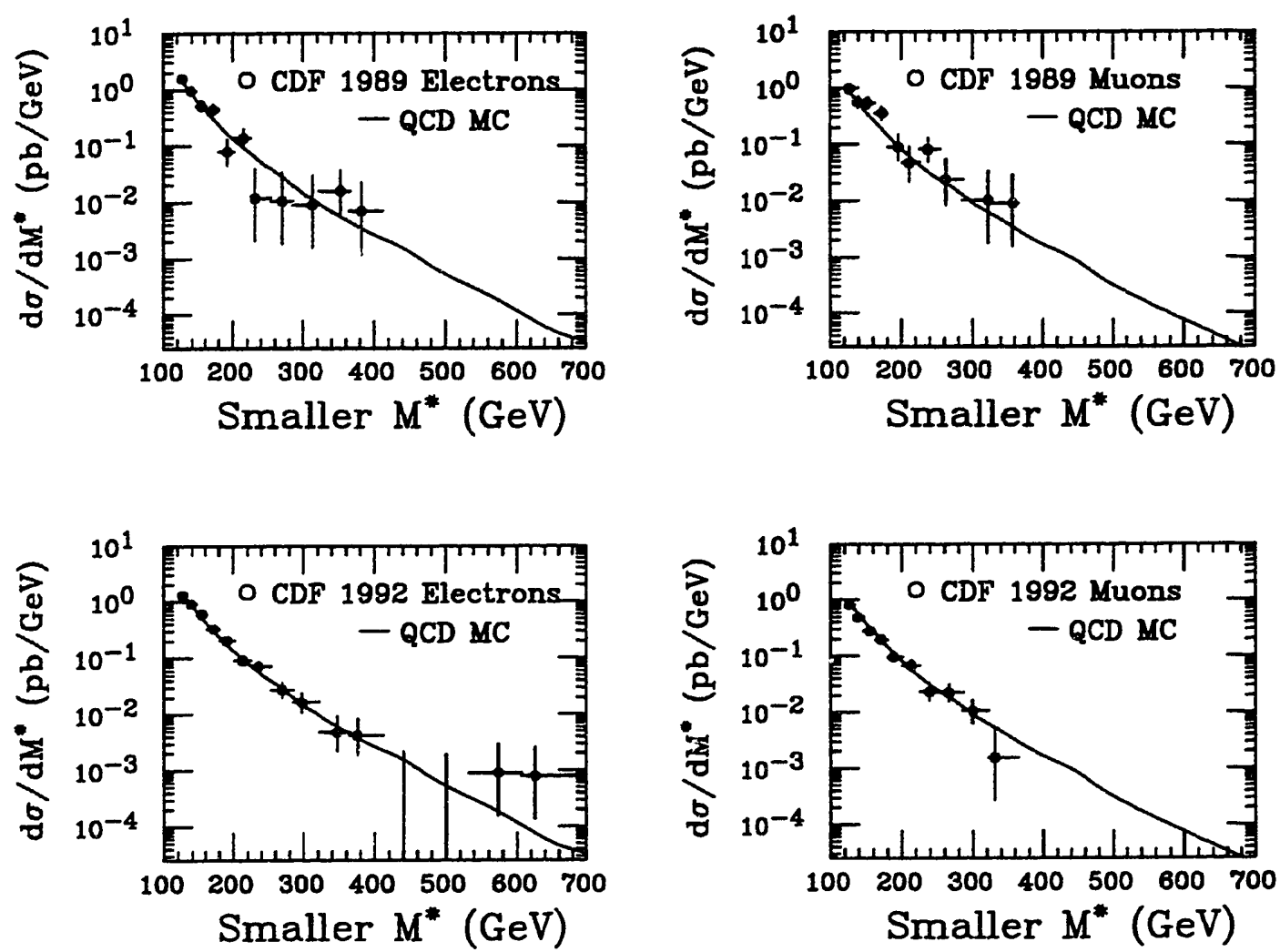

Figure 11.4: The selected (smaller) $M^{*}$ distribution for tho 1989 electron, 1989 muon, 1992 electron, and 1992 muon samples separately. The curves are the VECBOS-QDJTMC prediction normalize $\mathfrak{i}$ to the number of events in the combined data sample. 


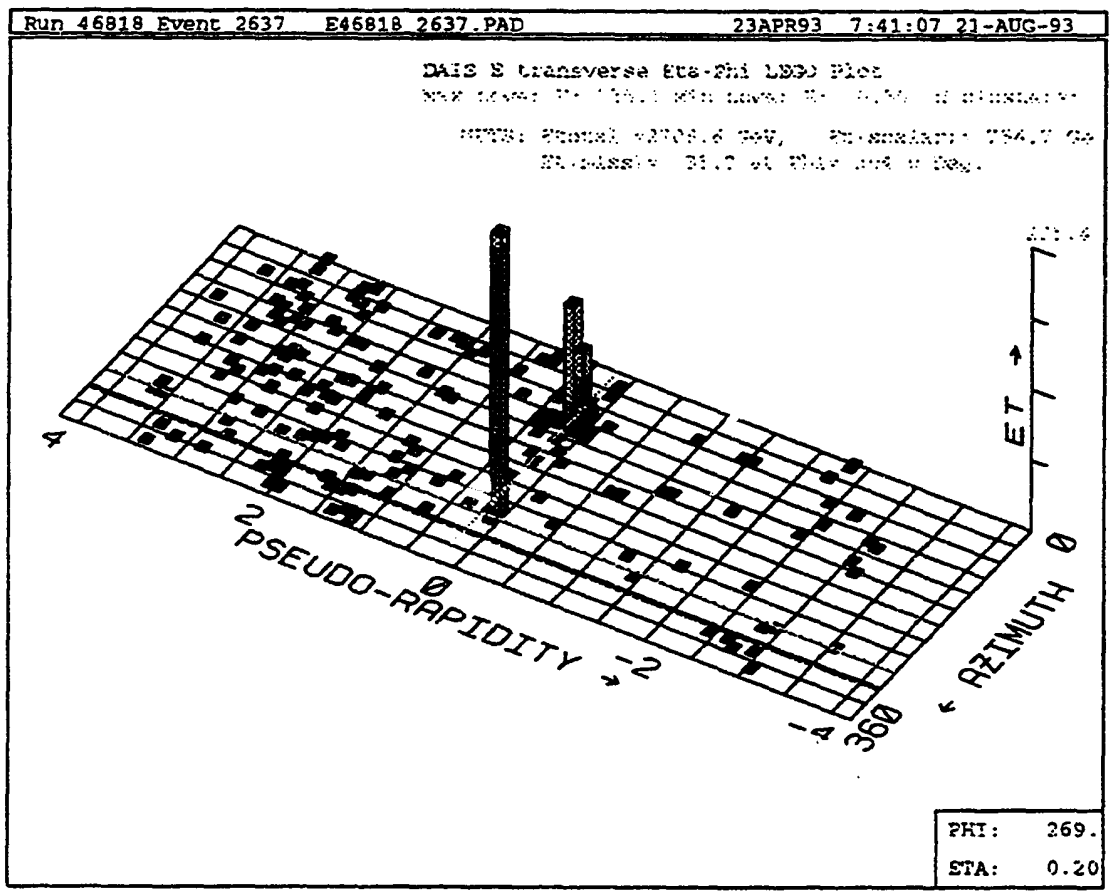

Figüre 11.5: The calorimeter energy deposition for the highest mass event. The calorimeter has been "unrolled" to make this lego plot. Energy in the electromagnetic calorimeters is shown darker than the hadronic energy. 


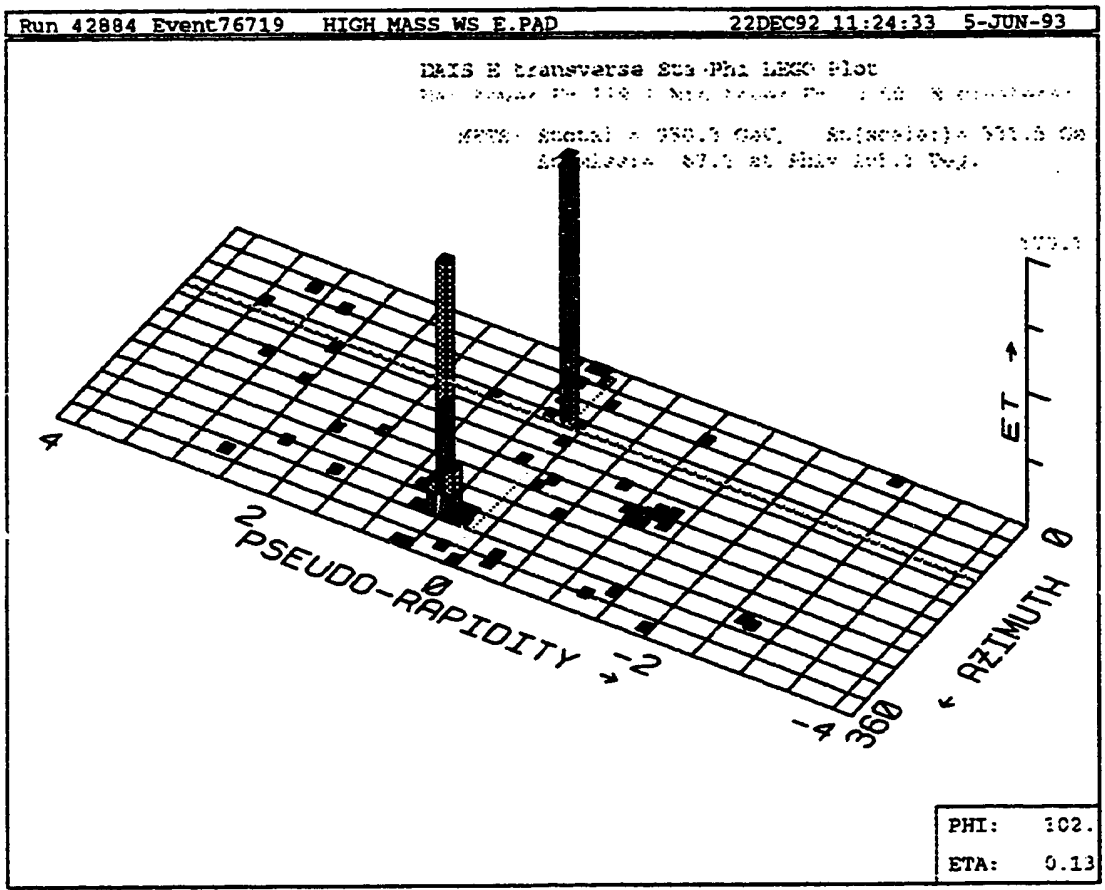

Figure 11.6: The calorimeter energy deposition for the 2nd highest mass event. The calorimeter has been "unrolled" to make this lego plot. Energy in the electromagnetic calorimeters is shown darker than the hadronic energy. 
QSTAR prediction normalized by a factor $\alpha$. That is, the predicted number of events in bin $i$ would be:

$$
\mu_{i}=a N_{i}(V E C B O S)+\alpha N_{i}(Q S T A R) .
$$

Where $N_{i}$ (VECBOS) is the VECBOS prediction for the number of events in bin $i$, and $N_{i}$ (QSTAR) is the prediction from QSTAR. The factor $a$ is used to make the total number of events in the prediction equal to the total number of events in the data; however, $a$ is not allowed to become smaller than zero. In other words, the normalization of VECBOS is allowed to vary in order to maximize the likelihood function (because the normalization of the VECBOS prediction is more uncertain than it's prediction of the shape for the $\mathrm{M}_{s}^{*}$ distribution).

The likelihood, $L(\alpha)$, is then the (Poisson) probability that this prediction produced the number of events measured in the CDF data. That is:

$$
L(\alpha)=\prod_{i} \frac{\mu_{i}^{n_{i}} e^{-\mu_{i}}}{n_{i} !}
$$

where $n_{i}$ is the number of events in bin $i$ in the data.

The $95 \%$ confidence limit can then determined by finding the value of $\alpha_{\text {Limit }}$ such that

$$
\frac{\int_{0}^{\alpha_{\text {Limit }}} L(\alpha) d \alpha}{\int_{0}^{\infty} L(\alpha) d \alpha}=0.95 .
$$

The excited quark cross section limit is then $\alpha_{\text {Limit }}$ times the cross section generated by the QSTAR Monte Carlo (before any cuts were placed on the events, and before detector simulation).

The electron and muon distributions were kept separate during this procedure, but the same normalization factors $(a$ and $\alpha$ ) were applied to both distributions simultaneously. Doing this takes into account the different number of events in the two channels and makes the limit more powerful. In practice, however, the number of events in both channels are similar (to within $30 \%$ ) and so this method changes the limit very little.

This procedure was repeated for eleven different values of $M_{q^{*}}$ (starting at 150 $\mathrm{GeV}$ and going up in $50 \mathrm{GeV}$ increments to $650 \mathrm{GeV}$ ) to obtain a plot of the limit versus mass. The lowest $q^{*}$ mass searched for in this analysis is $150 \mathrm{GeV}$. This value was chosen so that the $q^{*}$ state and the $\mathrm{W}$ boson could fluctuate several $\sigma$ off-shell and still be accepted by all the analysis cuts (especially the $15 \mathrm{GeV}$ jet $\mathrm{P}_{t}$ cut). As an illustration, figure 11.7 shows the likelihood function, $L(\alpha)$, for the $550 \mathrm{GeV}$ excited quark prediction. The $95 \%$ integral is shown by the vertical line.

Section 11.7 will discuss the systematic uncertainties in this measurement, and how they were included in the determination of the cross section limits. After the systematic uncertainties are included, the final limit results will be shown. 


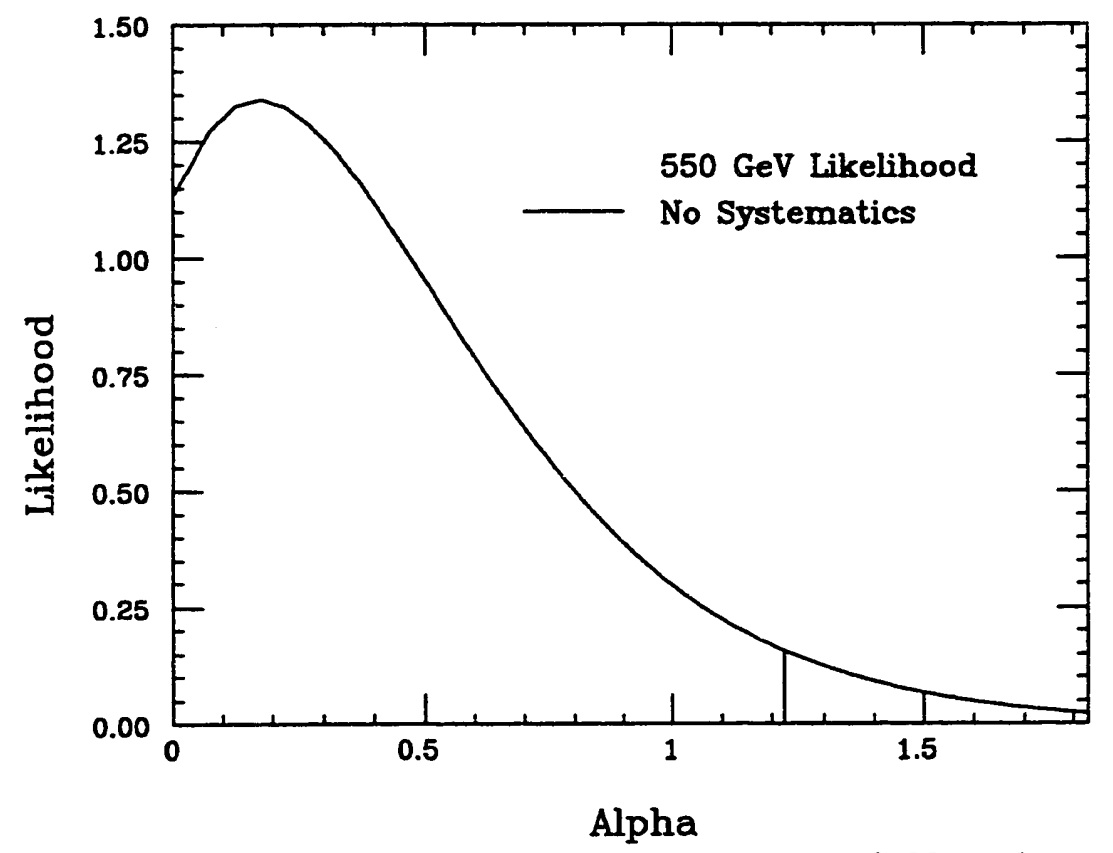

Figure 11.7: The likelihood function for a $q^{*}$ of mass $550 \mathrm{GeV}$, without systematic uncertainties. The vertical line, at 1.22 , shows the $95 \%$ confidence limit (the $95 \%$ integral). 


\subsection{Systematics}

The systematic uncertainties on the $q^{*}$ limits include all of the systematics discussed in the $\cos \theta_{s}^{*}$ analysis (see Chapter 9) as well as uncertainties on the integrated luminosity and the event selection efficiencies. This section first describes the method that was used to include the systematics in the limit calculation, and then discusses each of the systematic uncertainties. The section concludes with a summary of the systematic uncertainties.

\subsubsection{Method for Folding Systematics into the Limits}

The effects of the various systematic uncertainties on the cross section limits are estimated using a method similar to that used for CDF's top quark search[2].

First, each systematic uncertainty will be expressed as an uncertainty on the parameter $\alpha$-see equation 11.2 (in section 11.6). The uncertainty on $\alpha$ is taken to be the change in the $95 \%$ confidence limit, $\alpha_{\text {Limit }}$, when the experimental quantity (e.g. luminosity) is varied by its expected uncertainty. The change in $\alpha_{\text {Limit }}$ will be determined from Monte Carlo events, not the CDF data (this will be discussed more below).

Once the uncertainty on $\alpha$ is known for each systematic, they are all added together in quadrature to obtain a combined uncertainty on $\alpha, \sigma_{\alpha}$ (one for each $q^{*}$ mass). Given the total uncertainty on $\alpha$, a new likelihood function $\left(L^{\prime}\right)$ is formed by smearing the old likelihood function $(L)$ with a gaussian of width $\sigma_{\alpha}$ :

$$
L^{\prime}(\alpha)=\int_{0}^{\infty} L(x) \frac{1}{\sqrt{2 \pi} \sigma_{\alpha}} e^{-\frac{1}{2}\left(\frac{\alpha-x}{\sigma_{\alpha}}\right)^{2}} d x .
$$

The new $95 \%$ confidence cross section limit, $\alpha_{\text {Limit }}^{\prime}$, is determined using $L^{\prime}$ :

$$
\frac{\int_{0}^{\alpha_{\text {Limit }}^{\prime}} L^{\prime}(\alpha) d \alpha}{\int_{0}^{\infty} L^{\prime}(\alpha) d \alpha}=0.95
$$

Note that the normalizations of $L$ and $L^{\prime}$ are different, because values of $\alpha$ less than zero were not considered when smearing $L$ into $L^{\prime}$ (these values of $\alpha$ are unphysical).

Some uncertainties could have been smeared using a gaussian with a width that depended on $\alpha$. For example, an uncertainty on the integrated luminosity could be smeared in with a gaussian of width $\sigma_{\alpha} / \alpha_{\text {Limit }} \cdot \alpha$, which goes to zero when $\alpha=0$. However, for other uncertainties (which change the shape of the $\mathrm{M}_{s}^{*}$ distribution rather than just its overall normalization) it is more appropriate to use a fixed width gaussian. It is more conservative to use a fixed width gaussian 
instead of a variable width one, and since it is simpler to use only one approach, the fixed width gaussian is used for all systematics.

The description of this treatment of the systematics may be confusing, so a simple example is probably in order. Consider the luminosity uncertainty, which is $7 \%$. The uncertainty on $\alpha$ does not need to be determined using Monte Carlo events, it will just be $7 \%$. Suppose that this is the only uncertainty, and that we want to evaluate its effect on the cross section limit for a $500 \mathrm{GeV} q^{*}$. The $95 \%$ confidence limit on $\alpha$, for a $500 \mathrm{GeV} q^{*}$ without systematic uncertainties, is 0.49 , so the likelihood function will be smeared with a gaussian of width $\sigma_{\alpha}=$ $7 \% \cdot 0.49=0.034$.

\subsubsection{Monte Carlo Limit Predictions}

In order to evaluate the effect of systematic uncertainties (and for other studies described in section 11.8.1) a method was devised for using the Monte Carlo to predict how the cross section limits change under varying conditions. The VECBOS-QDJTMC prediction for the $\mathrm{M}_{s}^{*}$ distribution was first normalized to have the same total number of events as the data. This distribution was then used to generate 100 fake data samples, by choosing the number of events in each bin from a Poisson random number distribution (with average equal to the VECBOS prediction). The cross section limit was then evaluated for each of these 100 fake data samples, and the average value of the limits was taken as the Monte Carlo estimate for the limit. Figure 11.8 shows the distribution of the $95 \%$ confidence limit on $\alpha$, for the 100 fake samples, for a $500 \mathrm{GeV} q^{*}$. These runs were made using the default Monte Carlo events (i.e. without any alterations for studying systematics). The average of this distribution is 1.04 with standard deviation 0.33 .

\subsubsection{Discussion of Each Systematic Uncertainty}

The following sections describe each systematic uncertainty in detail, and the uncertainty it induces on $\alpha$. All of the systematics will then be combined and used to smear the likelihood function. The reader should keep in mind that the ultimate effect of all the systematics is to change the cross section limits by only $10 \%$ and the mass limit on $q^{*}$ by only $1 \%$ (i.e. including the systematic uncertainties is a small effect). Table 11.4 shows a summary of all the systematic uncertainties, as a function of $q^{*}$ mass; it can be referred to while reading the discussion of each uncertainty. 


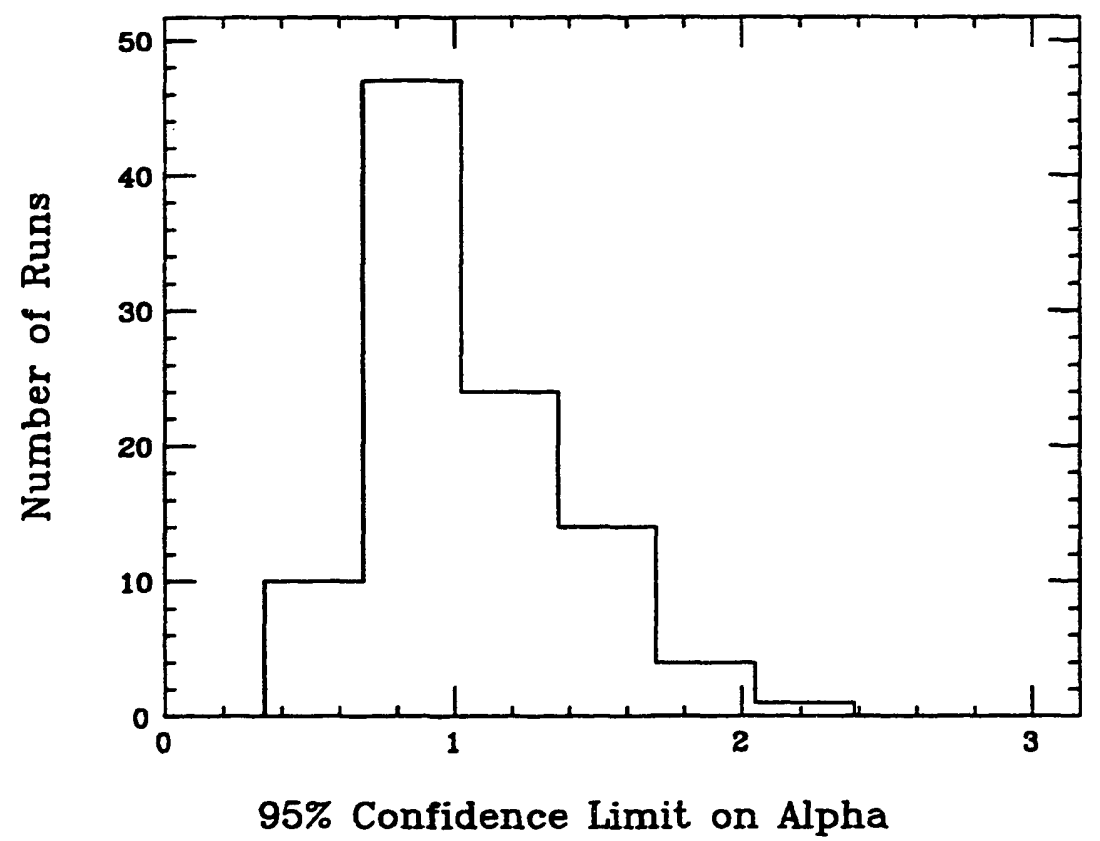

Figure 11.8: A histogram showing the results of 100 runs to determine the Monte Carlo predicted limit on $\alpha$. 


\section{Luminosity}

The estimates for the integrated luminosity in the CDF data samples is uncertain to $\pm 7 \%$. This translates into a $7 \%$ uncertainty on $\alpha$ for all $q^{*}$ masses.

\section{Event Selection Efficiency}

The uncertainties on the efficiencies for the electron and muon event selection cuts were shown in tables 11.2 and 11.3. The total uncertainty, for both muons and electrons, is $5 \%$. This uncertainty is taken to be constant as a function of $q^{*}$ mass.

This assumption is supported by studies made in the $P_{t}$ measurement for the $W[35]$, which showed little dependence of the efficiencies on the $P_{t}$ of the W boson. The two factors that may be mass dependent are the Had/EM cut, which was corrected for as described in section 11.4.1.3, and the lepton isolation. Since the lepton and jet are required to be separated in $\eta-\phi$ space, the isolation should be modeled correctly by the Monte Carlo.

\section{Absolute Jet Energy Scale}

The uncertainty on the jet energy scale was discussed in section 9.1.1, and is taken to be $5 \%$. In order to evaluate the effect of this uncertainty on $\alpha, q^{*}$ events were simulated with the jet energy scale lowered by $5 \%$. The $5 \%$ decrease in the jet energy scale typically shifted the $q^{*}$ mass peak down by about $4 \%$.

The Monte Carlo method was then used to estimate the change in the $95 \%$ confidence limit. In order to conserve CPU time, this was done only for the $q^{*}$ masses $150,300,500$, and $650 \mathrm{GeV}$. The change in the limit varied from $15 \%$ at $150 \mathrm{GeV}$ to $8 \%$ at $650 \mathrm{GeV}$. The change in the limit, judging from the four masses checked, decreases linearly with mass; therefore, the uncertainties for the other mass values were interpolated. Table 11.4 shows the uncertainty used for each $q^{*}$ mass. This scale uncertainty is the dominant systematic uncertainty.

The effect of the scale uncertainty was evaluated using only the electron distributions. This was done in order to make the CPU requirements of the estimates reasonable. It was also done because the only significant difference between the muon and electron events is that the muon events have a lepton $\eta$ cut of 0.6 instead of 0.9 . The difference in the $\eta$ cuts should not effect the uncertainties. Unless otherwise stated, all further systematics were evaluated using only the electron Monte Carlo events. 


\section{Relative Jet Energy Scale}

The relative scale of the piing and forward calorimeters are uncertain by about $2 \%$, as discussed in section 9.1.2. The effect on $\alpha$ was evaluated using the Monte Carlo method with $q^{*}$ events simulated with the plug and forward jet response reduced by $2 \%$.

The change in $\alpha$ was less than $1.5 \%$ for all $q^{*}$ masses tested $(150,300,500$, and $650 \mathrm{GeV}$ ). Since only 100 runs were made with the Monte Carlo, the predictions should fluctuate by less than $1 \%$. To be conservative, the uncertainty due to the relative energy scales will be taken to be $2 \%$ for all mass values.

\section{Jet Energy Resolution}

The uncertainty on the jet energy resolution was determined[25], using the dijet balancing methods described in section 5.3 .1 , to be $\pm 10 \%$. This uncertainty is dependent on the $\eta$ of the jet, but a fixed $10 \%$ was used because it brackets the uncertainties.

To estimate the effect on $\alpha$, the Monte Carlo method was used again, for the four test $q^{*}$ masses, using $q^{*}$ signal events simulated with the jet resolution increased by $10 \%$. Increasing the jet resolution by $10 \%$ increased the $q^{*}$ mass resolution by about $4 \%$. The limits all changed by less than $1.5 \%$; therefore, the uncertainty is taken to be $2 \%$ for all inasses.

\section{$q^{*}$ Transverse Momentum}

The QSTAR Monte Carlo produces $q^{*}$ states with no transverse momentum. In order to test how sensitive the limit is to the $\mathrm{P}_{t}$ of the $q^{*}$, a transverse boost was applied to the events. The $q^{*}$ was given a transverse momentum generated from a gaussian distribution with average zero and width equal to $1 / 10$ th the $q^{*}$ mass[37].

After applying the large $\mathrm{P}_{t}$ lick to the $q^{*}$ events, there was no significant change in the position, width, or acceptance of the $q^{*}$ signal. Therefore, it was judged unnecessary to run the Monte Carlo limit calculation for these events, and simply take the uncertainty to be the minimum $2 \%$ for all $q^{*}$ masses.

\section{Missing $E_{t}$ Resolution}

The most significant contribution to the missing- $E_{t}$ resolution is probably the jet resolution, which was described above. Tie next most significant effect would be the underlying event nodel. QDJTMC's underlying event model was described 
in section 6.1.2. During the 1992 run, the instantaneous luminosity was significantly higher than it was during the 1989 run; therefore, it was more probable for overlapping events to occur (events in which more than one $p \bar{p}$ collision occurred in a single beam crossing). The effect of these overlapping events is to increase the average total energy in the calorimeters. The QDJTMC simulation generates events with average $\sum E_{t}$ of $27 \mathrm{GeV}$ and width $18 \mathrm{GeV}$. A better fit to the 1992 data is an average of $44 \mathrm{GeV}$ and a width of $29 \mathrm{GeV}$. The 1992 values were used to simulate the $q^{*}$ events in order to estimate the systematic uncertainty due to the underlying event.

Raising the underlying event energy did not significantly change the position, width, or acceptance for the $q^{*}$ signal; therefore, the systematic uncertainty on $\alpha$ is taken to be the minimum $2 \%$ for all $q^{*}$ masses.

\section{Parton Distribution Functions}

The acceptance for $q^{*}$ events could change if different parton distribution functions were used to generate the events. The default pdf used was CTEQ1L. Two other pdf's were used to evaluate this systematic, HMRSB and MT-B2 (these were discussed in section 9.3). The acceptance for each pdf set was defined to be the cross section for the QSTAR events entering the final event sample over the cross section generated by the Monte Carlo.

The systematic uncertainty, for each $q^{*}$ mass, is taken to be the largest variation among the pdf sets. The systematic ranges from $6 \%$ at $150 \mathrm{GeV}$ to $0.5 \%$ at 650 ; however, $2 \%$ will be used as the minimum. This systematic was also evaluated at four $q^{*}$ masses $(150,300,500$, and $650 \mathrm{GeV})$ and was interpolated for the other mass values. See table 11.4 for the systematic uncertainty used for each mass.

\section{Q Scale}

The $q^{*}$ prediction is a leading order calculation, so it will be sensitive to the $\mathrm{Q}$ scale used (see section 9.4). The default scale was the dynamic mass of the $q^{*}$ state, $\mathbf{M}_{q^{*}}$. In order to estimate this systematic, the $q^{*}$ events were generated using $M_{q^{*}} / 2$ for the scale. The change in acceptance was less than $2 \%$ for all masses; therefore, the minimum $2 \%$ was used.

\section{Backgrounds}

When determining the cross section limits, the VECBOS prediction was used to represent the data. The backgrounds expected in the sample are small and have $\mathrm{M}^{*}$ distributions that are similar to the VECBOS $\mathrm{M}^{*}$ distribution; therefore, the 
VECBOS distribution alone was a reasonable choice. In this section, the backgrounds in the sample are estimated, and the difference in the limit caused by including the backgrounds with the VECBOS prediction is used as a systematic uncertainty. This was done this way because the background that changes the limit the most is the dijet background, which is also the background prediction in which the least confidence can be placed.

Since the dijet background is the dominate effect, only the electron events were used to study the effect of backgrounds. The method used to estimate the dijet events gives a larger systematic uncertainty for the electron data than it does for the muon data, so using only the electron events is conservative.

The backgrounds in the sample were estimated in the same way as for the $\cos \theta_{s}^{*}$ distribution (see Chapter 8). Pythia-QFL was used to generate $W \rightarrow \tau \nu$, $Z \rightarrow e e$, and $Z \rightarrow \tau \tau$ events. The fraction of these backgrounds in the data was predicted by generating $W \rightarrow e \nu$ events with Pythia and normalizing all four contributions to match the total number of events in the data with a transverse mass greater than $20 \mathrm{GeV}$. The transverse mass cut was used, because most of the dijet background is expected to have low transverse mass. The sample is estimated to contain $4.7 \% \mathrm{~W} \rightarrow \tau \nu, 2.0 \% Z \rightarrow e e$, and $1.1 \% Z \rightarrow \tau \tau$.

The QCD background contamination is then taken to be the excess events with transverse mass less than $20 \mathrm{GeV}$. Figure 11.9 shows the transverse mass distribution compared to the total electroweak prediction. The excess makes up $6.6 \%$ of the total sample.

Now that the QCD contamination has been estimated, the shape of its $M_{s}^{*}$ spectrum must be predicted. This was done using the dijet Monte Carlo described in section 8.2. Both method I and method II were used to generate dijet backgrounds. The method I sample (in which the electron energy was divided by 2 before detector simulation) causes the largest systematic uncertainty; therefore, it will be used from here on.

The $\mathrm{M}_{s}^{*}$ distributions for VECBOS, the Pythia electroweak backgrounds, and the dijet prediction were added together and compared to the $M_{s}^{*}$ for just VECBOS. Figure 11.10 shows the ratio of the $\mathrm{M}_{s}^{*}$ distribution for the combined backgrounds to that for the pure VECBOs prediction. There is a lowering of the $\mathrm{M}_{s}^{*}$ curve at high mass, and a peak at about $200 \mathrm{GeV}$. The peak comes from the dijet background (if the method II dijet background had been used, there would still be a peak, but it would be about $1 / 2$ the size).

The Monte Carlo method was then used to estimate the change in the limits caused by including the backgrounds. The limits were estimated at the $q^{*}$ mass values $150,200,250,300,400,500$, and $650 \mathrm{GeV}$ (to obtair a more dense sampling around the $200 \mathrm{GeV}$ peak). The uncertainty in $\alpha$ ranges from $13 \%$ at $150 \mathrm{GeV}$ to $2 \%$ at $650 \mathrm{GeV}$, and has some structure around the peak. See table 11.4 for the uncertainty used for each mass value. 


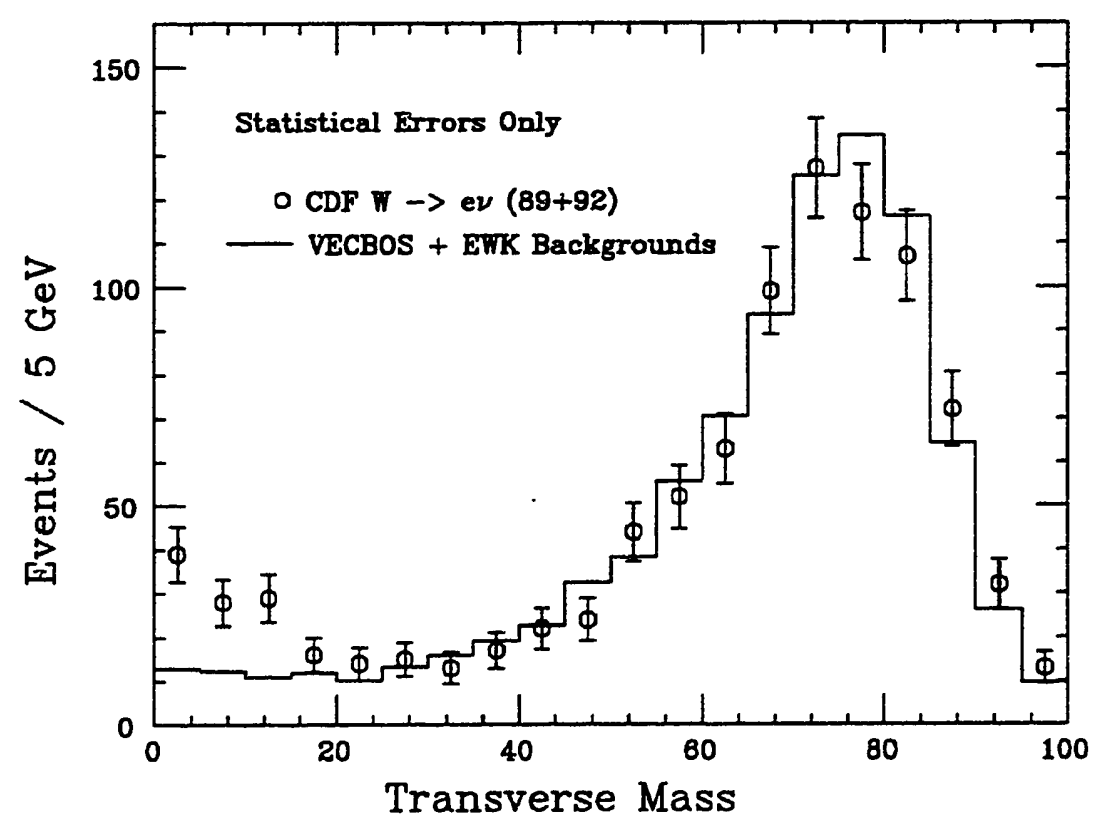

Figure 11.9: The transverse mass of the $W$ boson for the 1989 and 1992 electron samples. The histogram is the vecBos prediction combined with Pythia $W \rightarrow \tau \nu$, $Z \rightarrow \epsilon e$, and $Z \rightarrow \tau \tau$, normalized to the data above $20 \mathrm{GeV}$.

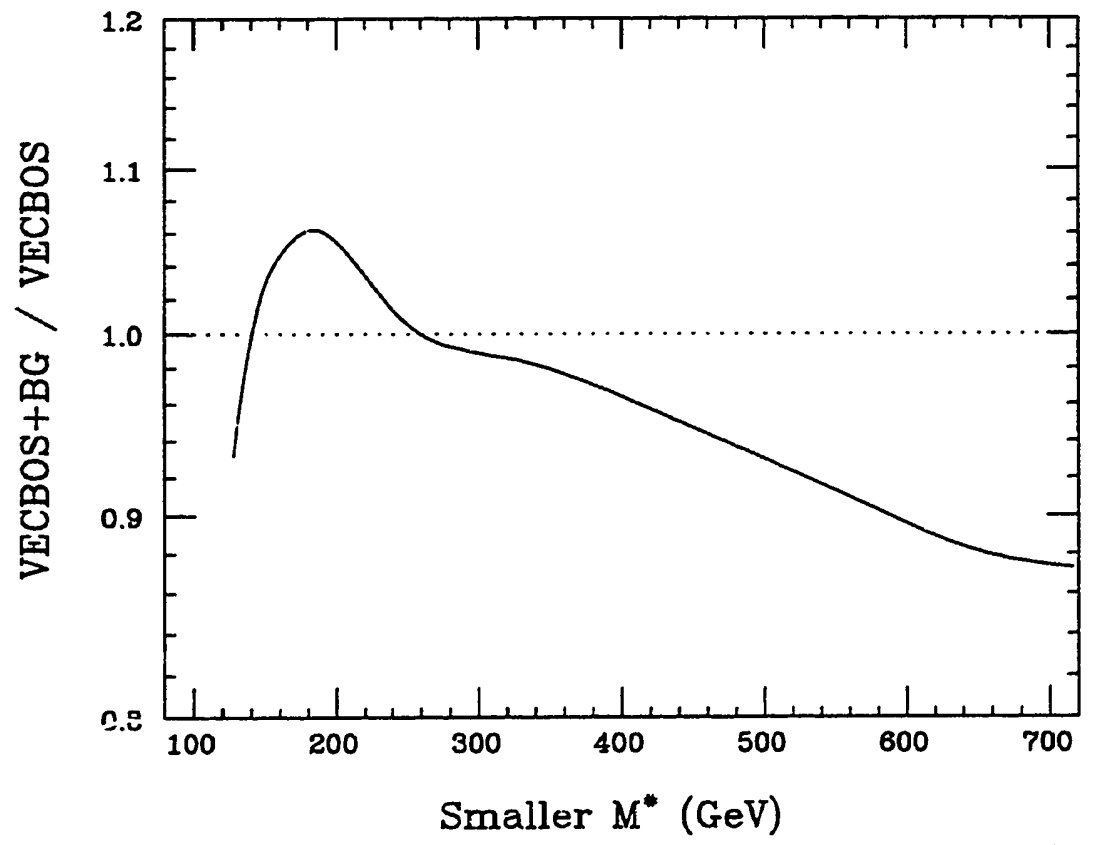

Figure 11.10: The ratio of the $M^{*}$ distribution for VECBOs and all backgrounds (including dijets) to the $\mathrm{M}^{*}$ distribution for just $\vee$ Eсвоs. 


\begin{tabular}{||l|r|r|r|r|r|r|r|r|r|r|r||}
\hline Systematic & \multicolumn{10}{|c||}{ Uncertainty in $\alpha$ (in percent) } \\
Uncertainty & \multicolumn{10}{|c|}{$q^{*}$ Mass } \\
& 150 & 200 & 250 & 300 & 350 & 400 & 450 & 500 & 550 & 600 & 650 \\
\hline Luminosity & 7 & 7 & 7 & 7 & 7 & 7 & 7 & 7 & 7 & 7 & 7 \\
\hline Efficiency & 5 & 5 & 5 & 5 & 5 & 5 & 5 & 5 & 5 & 5 & 5 \\
\hline Abs. Jet Scale & 15 & 14 & 13 & 12 & 12 & 11 & 10 & 9 & 9 & 8 & 8 \\
\hline Rel. Jet Scale & 2 & 2 & 2 & 2 & 2 & 2 & 2 & 2 & 2 & 2 & 2 \\
\hline Jet Resolution & 2 & 2 & 2 & 2 & 2 & 2 & 2 & 2 & 2 & 2 & 2 \\
\hline$q^{*} P_{t}$ & 2 & 2 & 2 & 2 & 2 & 2 & 2 & 2 & 2 & 2 & 2 \\
\hline Underlying E & 2 & 2 & 2 & 2 & 2 & 2 & 2 & 2 & 2 & 2 & 2 \\
\hline PDF & 6 & 6 & 5 & 5 & 4 & 3 & 2 & 2 & 2 & 2 & 2 \\
\hline$Q$ Scale & 2 & 2 & 2 & 2 & 2 & 2 & 2 & 2 & 2 & 2 & 2 \\
\hline Backgrounds & 13 & 9 & 10 & 14 & 14 & 12 & 4 & 2 & 2 & 2 & 2 \\
\hline \hline Combined & 23 & 20 & 20 & 21 & 21 & 19 & 15 & 14 & 14 & 13 & 13 \\
\hline \hline Limit Change & 13 & 11 & 10 & 12 & 12 & 11 & 9 & 7 & 6 & 5 & 5 \\
\hline
\end{tabular}

Table 11.4: Summary of systematic uncertainties. The values in the table are the percentage uncertainty on $\alpha$. The "Limit Change" row shows the percent change in the $95 \%$ limit on $\sigma_{q^{*}} \cdot B_{W J}$ when the combined systematic uncertainty is used to smear the likelihood function.

\subsubsection{Summary of Systematic Uncertainties}

Table 11.4 summarizes the systematic uncertainties used to smear the likelihood functions. The individual uncertainties are combined, in quadrature, to obtain a total uncertainty on $\alpha$. The total uncertainty ranges from $23 \%$ at $150 \mathrm{GeV}$ to $13 \%$ at $650 \mathrm{GeV}$. After smearing the likelihood function, the $95 \%$ cross section limits changed by $13 \%$ at $150 \mathrm{GeV}$ to $5 \%$ at $650 \mathrm{GeV}$. 11.11 shows the likelihood functions, for a $550 \mathrm{GeV} \mathrm{q}$, before and after the smearing for systematic uncertainties.

\subsection{Excited Quark Limits}

Figure 11.12 shows the $95 \%$ confidence limits on $\sigma_{q^{*}} \cdot B_{W J}$ as a function of the $q^{*}$ mass. These limits are relatively free of any assumptions about the theory; therefore, predictions from other theoretical models (e.g. something other than excited quark states) could be compared directly to these limits. The only assumptions built into these limits were that the width of the resonance is small compared to the mass resolution of the detector (which is $13 \%$ ), that decays of the $q^{*}$ and 


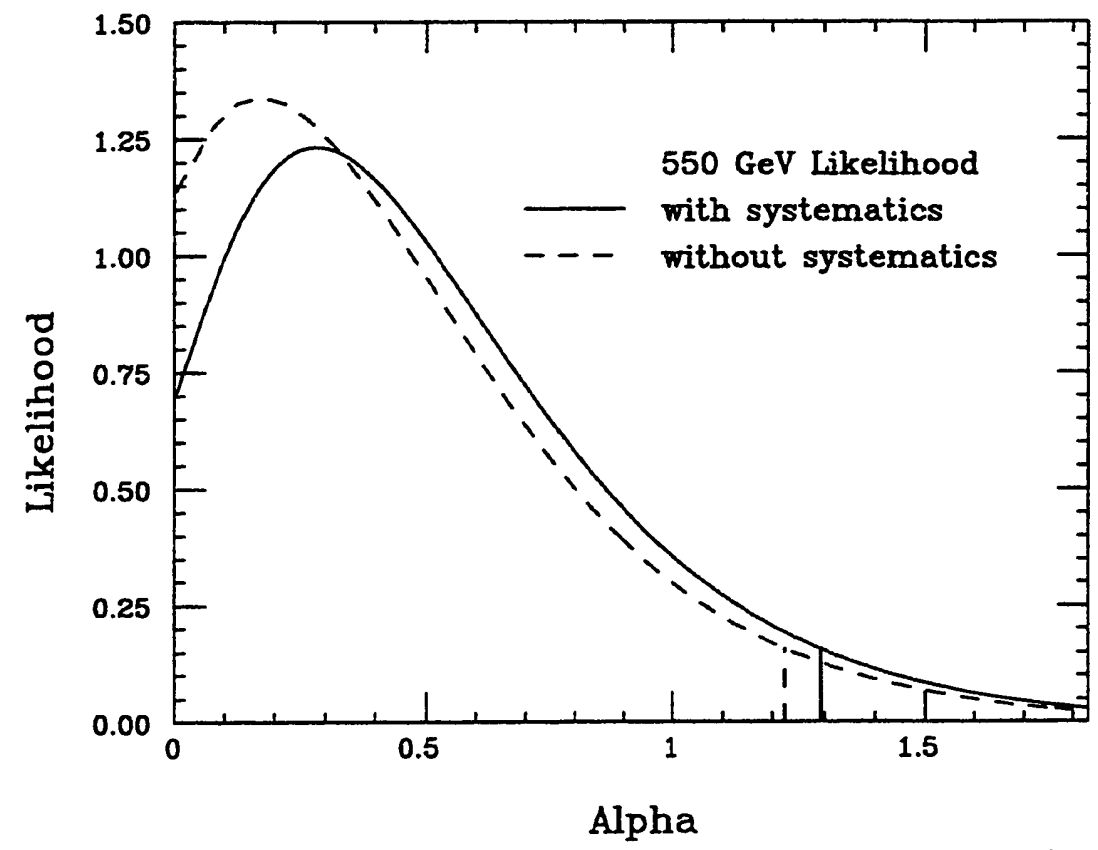

Figure 11.11: The likelihood functions for a $q^{*}$ of mass $550 \mathrm{GeV}$. The solid curve is $L^{\prime}$, the likelihood with systematic uncertainties folded in. The dashed curve is $L$, the likelihood without systematics. The vertical lines, at 1.22 and 1.29 , show the $95 \%$ confidence limits. 
the $\mathrm{W}$ boson be isotropic, and that the initial state consisted of a quark and a gluon. The latter assumption only matters if the boost (along the $\mathrm{z}$-direction) of the $q^{*}$ is significantly different in some new theory (which might change the overall acceptance).

Also shown in figure 11.12 is the cross section predicted by the QSTAR Monte Carlo[28]. The point at which the two curves cross gives the mass limit on excited quark states. The limit is $530 \mathrm{GeV}$ (rounding down from roughly $534 \mathrm{GeV}$ ). The net result of the systematic uncertainties was to lower the mass limit by $5 \mathrm{GeV}$.

The $q^{*}$ cross section depends on the square of the coupling (i.e. $\sigma\left(q g \rightarrow q^{*} \rightarrow\right.$ $W J) \sim f^{2}$ ), so it is possible to quote the mass limit as a function of the coupling by multiplying the $q^{*}$ cross section by $f^{2}$. Figure 11.13 shows the region, in $M_{q^{*}}-f$ space, excluded at the $95 \%$ confidence level. Since the $q^{*}$ state becomes wider as $f$ gets larger $\left(\sigma_{q^{*}} \approx 0.04 f^{2} \mathrm{M}_{q^{*}}\right)$, the plot is only drawn out to the point where the coupling reaches 2.0. The QSTAR Monte Carlo was used to generate events with the coupling, $f$, set to 2.0 and 0.5 in order to verify that the change in width did not alter figure 11.13-there was no significant change.

\subsubsection{Mass Limit Studies}

This section is supplementary. It describes some tests that were made in order to check that the cuts used in the $q^{*}$ search were reasonably well optimized (because many of the cuts were simply carried over from the $\cos \theta_{s}^{*}$ measurement).

The Monte Carlo method (described in section 11.7.2) was used to estimate the sensitivity of the excited quark mass limits to changes in the analysis cuts. 50 fake data samples were generated for each test, and the average was used to estimate the limit. These tests were only done for one $q^{*}$ mass $-500 \mathrm{GeV}$ (because it was near the final mass limit).

The first test that was made was to try a maximum $\cos \theta_{s}^{*}$ cut of $2 / 3$ instead of 0.9 . The tighter cut should remove more QCD background events than it does the $q^{*}$ signal, which may improve the mass limit. When this tighter cut was used, the mass limit worsened by $1 \mathrm{GeV}$ (which is roughly the statistical uncertainty on the estimate).

Another test was made using the definition of the "selected" solution used in the $\cos \theta_{s}^{*}$ measurement, instead of using the smaller value of $\mathrm{M}^{*}$. When this choice was used, the limit worsened by $9 \mathrm{GeV}$. Choosing the smaller value of $M^{*}$ not only provides a more conservative way to present the $M_{s}^{*}$ distribution, it improves the mass limit. 


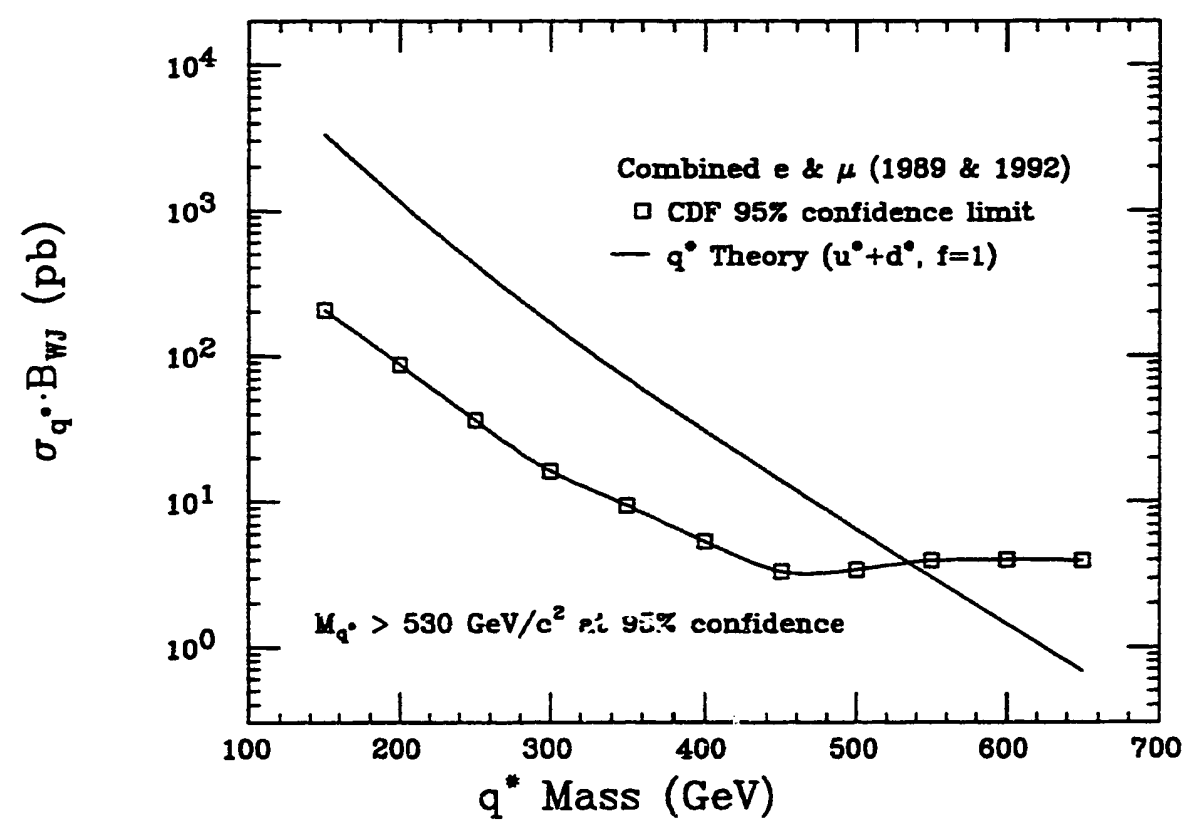

Figure 11.12: The 95\% confidence limit on $\sigma_{q^{*}} \cdot B_{W J}$. The curve is the QSTAR prediction using a coupling $f=1.0$, which gives a $95 \%$ confidence limit on $\mathbf{M}_{q^{*}}$ of 530 $\mathrm{GeV}$.

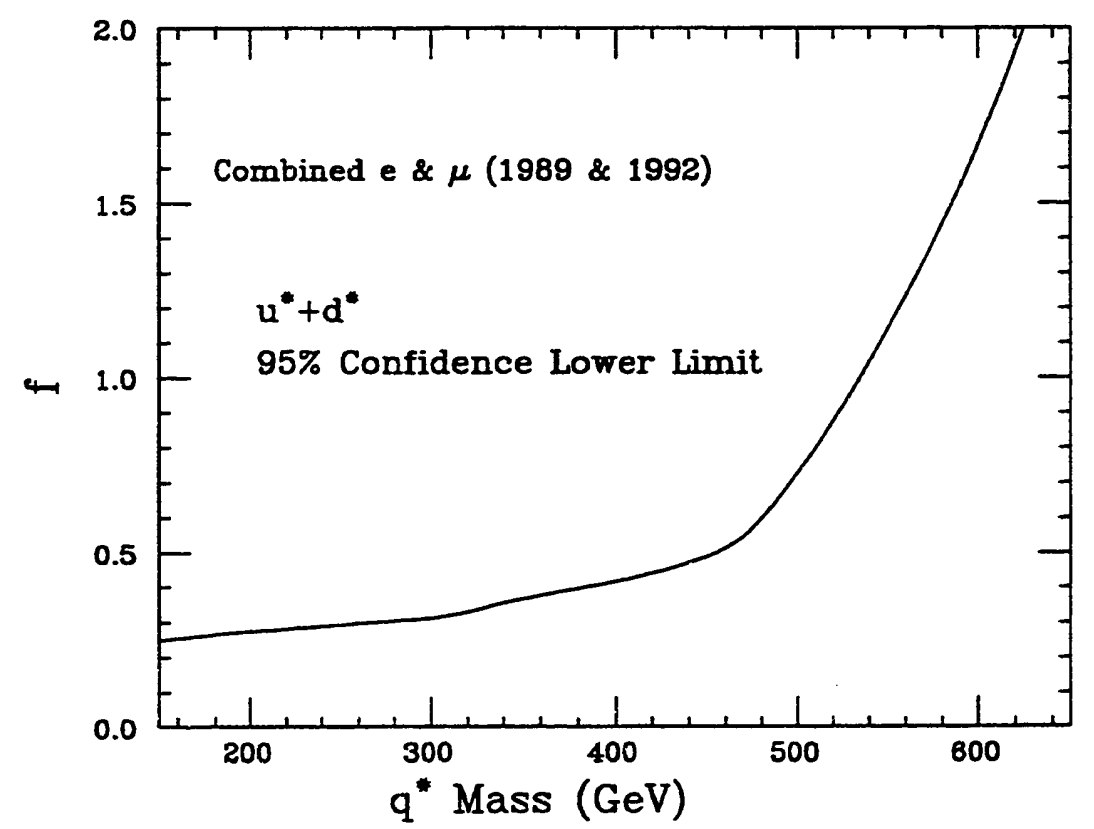

Figure 11.13: The $95 \%$ confidence limit on the QSTAR coupling constant, $f$, as a function of mass. The region above the curve is excluded at the $95 \%$ confidence level. 


\subsection{Conclusions}

The selected $\mathrm{M}^{*}$ distribution was used to place limits on $\sigma_{q^{*}} \cdot B_{W J}$ for excited quark states; these limits only rely on the assumptions that the width of the excited quark state is not much larger than what was used to make them $\left(\sigma_{q^{*}} \approx\right.$ $0.04 \mathrm{M}_{q^{*}}$ ), that the decay of the $q^{*}$ be isotropic, and that the boosts not be significantly different than those given by a quark-gluon initial state.

Degenerate $u^{*}$ and $d^{*}$ quarks with coupling $f=1$ are excluded, at $95 \%$ confidence, from the range $150<\mathrm{M}_{q^{*}}<530 \mathrm{GeV}$. This limit can be compared with previous limits reported by LEP $\left(\mathrm{M}_{q^{*}}<88 \mathrm{GeV}\right)$ and UA2 $\left(140<\mathrm{M}_{q^{*}}<288\right.$ $\mathrm{GeV}$ ). This limit has also been combined with CDF photon + jet events[38] to extend the CDF excluded range to $80<\mathrm{M}_{q^{*}}<540 \mathrm{GeV}$. Considering all of these results, this model for excited quarks can be excluded below $540 \mathrm{GeV}$. 


\section{Chapter 12}

\section{Conclusions}

$\mathrm{W}+$ jet events, produced in proton-antiproton collisions, provide an excellent environment for testing the Standard Model. Their unique signatures allow for the accumulation of very pure data samples, essentially free from background contamination. Two measurements have been presented based on these events.

The scattering angle in $\mathrm{W}+$ jet events has been measured using the electronic decay mode of the $\mathrm{W}$ boson in $4.05 \mathrm{pb}^{-1}$ of data recorded by CDF. The distribution of selected $\cos \theta^{*}$, shown in figure 10.1 , agrees very well with leading order and next-to-leading order QCD predictions. The distribution of selected $\left|\cos \theta^{*}\right|$, shown in figure 10.3, was compared directly with dijet and pnuitun + jet events recorded with CDF. The $\mathrm{W}+$ jet and photon + jet events show a scattering distribution consistent with a spin- $\frac{1}{2}$ (quark) propagator, while the dijet events clearly show a more peaked distribution consistent with a spin-1 (gluon) propagator.

The invariant mass of the $W+$ jet system has been used to place limits on the properties of excited quark states. Theories other than excited quark states can also be checked against the presented results. This search used the electronic decay mode of the $\mathrm{W}$ boson in $25.4 \mathrm{pb}^{-1}$ of data recorded by CDF, and the muonic decay mode of the $\mathrm{W}$ boson in $24.8 \mathrm{pb}^{-1}$ of CDF data. Limits on the cross section times branching ratio, $\sigma_{q^{*}} \cdot B_{W J}$, were shown in figure 11.12. These limits were used to exclude degenerate $u^{*}$ and $d^{*}$, at $95 \%$ confidence, from the range $150<\mathrm{M}_{q^{*}}<530 \mathrm{GeV}$ (which can be compared to the previous published limit of $288 \mathrm{GeV}$ at $90 \%$ confidence from UA2, using the quark + gluon decay mode of the $\left.q^{*}\right)$. The excited quark limits were also presented as an excluded region in the $f-\mathrm{M}_{q^{*}}$ plane, in figure 11.13 , because different compositeness models will undoubtedly predict different values for the coupling parameter $f$. 


\section{Bibliography}

[1] M. Aguilar-Benitez et al. Review of Particle Properties. Phys. Rev. D 45, 1 (1992).

[2] CDF Collaboration. Limit on the Top-Quark Mass from Proton-Antiproton Collisions at $\sqrt{s}=1.8$ TeV. Phys. Rev. D 45, 3921-3948 (1992).

[3] CDF Collaboration. Top Dilepton Search at CDF. Submitted to 16th International Symposium on Lepton and Photon Interactions, Ithaca, NY, 10-15 August 1993.

[4] Robert N. Cahn and Gerson Goldhaber. The Experimental Foundations of Particle Physics. Cambridge University Press, Cambridge, New York, 1989.

[5] CTEQ Collaboration. CTEQ Parton Distributions and Flavor Dependence of Sea Quarks. Physics Letters B304, 159-166 (1993).

[6] G. Altarelli and G. Parisi. Asymptotic Freedom in Parton Language. Nuclear Physics B126, 298 (1977).

[7] F.A. Berends, H. Kuijf, B. Tausk, and W.T. Giele. On the Production of a $W$ and Jets at Hadron Colliders. Nucl. Phys. B 357, 32-64 (1991).

[8] John C. Collins and Davison E. Soper. Angular Distribution of Dileptons in High-Energy Hadron Collisions. Phys. Rev. D 16, 2219-2225 (1977).

[9] Kaoru Hagiwara, Ken-ichi Hikasa, and Naoyuki Kai. Parity-Odd Asymmetries in W-Jet Events at Hadron Colliders. Physical Review Letters 52, 10761079 (1984).

[10] CDF Collaboration. Dijet Angular Distributions from $\bar{p} p$ Collisions at $\sqrt{s}=$ 1.8-TeV. Physical Review Letters 62, 3020 (1989).

[11] Richard D. Si.Denis. Dijet Angular Distributions in Proton-Antiproton Collisions at the Fermilab Tevatron. PhD thesis, Harvard University, December 1988 . 
[12] CDF Collaboration. The Center-of-Mass Angular Distribution of Prompt Photons Produced in p $\bar{p}$ Collisions at $\sqrt{s}=1.8-T e V$. Physical Review Letters 71, 679-683 (1993).

[13] Leslie F. Nakae. Direct Photon Center-of-mass Angular Distributions in Proton-Antiproton Collisions at $\sqrt{s}=1.8 \mathrm{TeV}$. PhD thesis, Brandeis University, April 1992.

[14] W.T. Giele, E.W.N. Glover, and David A. Kosower. Higher Order Corrections to Jet Cross-Sections in Hadron Colliders. Preprint FERMILAB-PUB-91100-T, Fermilab, 1992. (Fermilab Library.

[15] CDF Collaboration. The CDF Detector: An Overview. Nucl. Instr. and Meth. A271, 387-403 (1988).

[16] Brian L. Winer. The W Boson Transverse Momentum Spectrum in ProtonAntiproton Collisions at $\sqrt{s}=1.8 \mathrm{TeV}$. PhD thesis, University of California, Berkeley, February 1991.

[17] Javid N. Brown. A Search for Double Parton Interactions in ProtonAntiproton Collisions at $\sqrt{s}=1.8 \mathrm{TeV}$. $\mathrm{PhD}$ thesis, Harvard University, June 1989.

[18] CDF Collaboration. Measurement of the W-Boson mass in 1.8-TeV $\bar{p} p$ Collisions. Phys. Rev. D 43, 2070-2093 (1991).

[19] P. N. Harriman, A. D. Martin, W. J. Stirling, and R. G. Roberts. Parton Distributions Extracted from Data on Deep Inelastic Lepton Scattering, Prompt Photon Production and the Drell-Yan Process. Phys. Rev. D 42, 798-810 (1990).

[20] Ian Hinchliffe. The PAPAGENO Partonic Monte Carlo Program. Technical Report LBL-34372, Lawrence Berkeley Laboratory, 1993. Submitted to Workshop on Physics at Current Accelerators and the Supercollider, Argonne, IL, 2-5 Jun 1993.

[21] CDF Collaboration. Topology of Three-Jet Events in $\bar{p} p$ Collisions at $\sqrt{s}=$ $1.8 \mathrm{TeV}$. Phys. Rev. D 45, 1448-1458 (1992).

[22] CDF Collaboration. Study of Four-Jet Events and Evidence for Double Parton Interactions in p p Collisions at $\sqrt{s}=1.8 \mathrm{TeV}$. Phys. Rev. D 47, 48574871 (1993).

[23] Hans-Uno Bengtsson and Torbjorn Sjostrand. The Lund Monte Carlo for Hadronic Processes: Pythia version 4.8. Comput. Phys. Commun. 46, 43 (1987). 
[24] G. Marchesini et al. HERWIG: A Monte Carlo Generator for Simulating Hadron Emission Reactions with Interfering Gluons. Version 5.1. Comput. Phys. Commun. 67, 465-508 (1992).

[25] Brenna L. Flaugher. Measurement of $Q C D$ Jet Broadening in ProtonAntiproton Collisions at $\sqrt{s}=1.8 \mathrm{TeV}$. PhD thesis, Rutgers University, October 1989.

[26] J. Kwiecinski, A. D. Martin, W. J. Stirling, and R. G. Roberts. Parton Distributions at Small X. Phys. Rev. D 42, 3645-3659 (1990).

[27] Jorge G. Morfin and Wu-Ki Tung. Parton Distributions from a Global QCD Analysis of Deep Inelastic Scattering and Lepton Pair Production. Z. Phys. C 52, 13-30 (1991).

[28] U. Baur, I. Hinchliffe, and D. Zeppenfeld. Excited Quark Production at Hadron Colliders. Int. J. Mod. Phys. A 2, 1285 (1987).

[29] U. Baur, M. Spira, and P. M. Zerwas. Excited Quark and Lepton Production at Hadron Colliders. Phys. Rev. D 42, 815-824 (1990).

[30] E. J. Eichten, C. Quigg, I. Hinchliffe, and K. D. Lane. Super Collider Physics. Rev. Mod. Phys. 56, 579-707 (1984).

[31] U. Baur and K. H. Streng. Phenomenology of Composite Colored Weak Bosons. Z. Phys. C 30, 325 (1986).

[32] G. J. Gounaris and A. Nicolaidis. Production of Colored Weak Bosons at the $p \bar{p}$ Collider. Physics Letters 148B, 239 (1984).

[33] ALEPH Collaboration. Searches for New Particles in $Z$ Decays using the Aleph Detector. Phys. Rept. 216, 253-340 (1992).

[34] UA2 Collaboration. A Search for New Intermediate Vector Mesons and Excited Quarks Decaying to Two Jets at the CERN $\bar{p} p$ Collider. Nuclear Physics $B$ 400, 3-24 (1993).

[35] CDF Collaboration. Measurement of the $W \mathrm{P}_{t}$ Distribution in $\bar{p} p$ Collisions at $\sqrt{s}=1.8 \mathrm{TeV}$. Physical Review Letters 66, 2951-2955 (1991).

[36] CDF Collaboration. A Measurement of th: Production and Muonic Decay Rate of $W$ and $Z$ Bosons in p p Collisions at $\sqrt{s}=1.8 \mathrm{TeV}$. Physical Review Letters 69, 28-32 (1992).

[37] Ian Hinchliffe, private communication. Ian suggested using an average $P_{t}$ equal to $1 / 10$ th of the $q^{*}$ mass as a rough estimate, because this is roughly what we see for the $\mathrm{W}$ boson. 
174

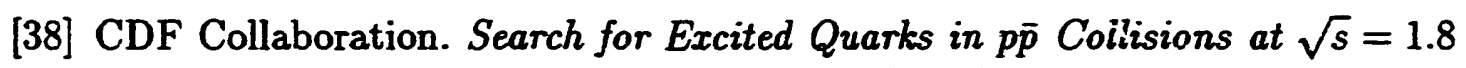
MeV. Submitted to Physical Review Letters, (1993).

ENd Date

$2-14-94$ 